\title{
SIMULATIONS OF SHEARING RHEOLOGY OF THERMOTROPIC LIQUID CRYSTALLINE POLYMERS
}

\author{
A Thesis \\ Presented to \\ The Graduate Faculty of The University of Akron
}

\author{
In Partial Fulfillment \\ of the Requirements for the Degree \\ Master of Science
}

Hongyan Chen

August, 2008 


\title{
SIMULATIONS OF SHEARING RHEOLOGY OF THERMOTROPIC LIQUID CRYSTALLINE POLYMERS
}

\author{
Hongyan Chen
}

Thesis

Approved:

Accepted:

Advisor

Dean of the College

Dr. Arkady I. Leonov

Dr. Stephen Z. D. Cheng

Faculty Reader

Dean of the Graduate School

Dr. Hendrik Heinz

Dr. George R. Newkome

Department Chair

Date

Dr. Sadhan C. Jana 


\begin{abstract}
The simulations present a first attempt to describe the rheological properties of the LCP's, using recently developed thermodynamic theory of weakly viscoelastic nematodynamics. In this theory the complicated rheological properties of nematic LCP's are modeled by a set of quasi-linear anisotropic viscoelastic constitutive equations (CE) with anisotropy described by director, whose viscoelastic evolution is coupled with the evolution of director. Although this theory has been developed for relatively small viscoelastic effects, it is still possible to compare the simulations with experimental data. A new mathematical tool, algebra of nematic operators, is helpful in operations with this multi-parametric theory. There are eight parameters in the theory which include 3 viscosities, 3 elastic moduli, and 2 tumbling (elastic and viscous) parameters. These parameters established for steady shearing are used for the calculations of evolution of shear stress and first normal stress difference with corresponding evolution of director, during relaxation and start up flow. The problem with initial conditions for director in start up flow is resolved in the following way. We preliminarily fitted the experimental data for stresses in steady shearing with following adjustment of parameters to describe also the relaxation of stresses. In this case the parameters of evolution equation for director, along with its orientation in steady shearing were also established. The orientation of director during stress relaxation was then easily calculated, and its final orientation at the rest state was taken as initial one for the start up flow. We demonstrate
\end{abstract}


that our simulations are at least in a semi-quantitative agreement with experimental data obtained for two industrial and two academic LCP's. Dependencies of fitted theoretical parameters on temperature and structure of LCP's have also been discussed. 


\section{ACKNOWLEDGEMENTS}

I would like to express deep appreciation to my advisor, Professor Arkady I.

Leonov during the course of the research leading to this thesis. His consistent support and guidance throughout the time of my study resulted in the success of this research.

Also, I would like to dedicate this thesis to my parents and my husband, Wenyi Huang, for their love, understanding, and support. 
TABLE OF CONTENTS

Page

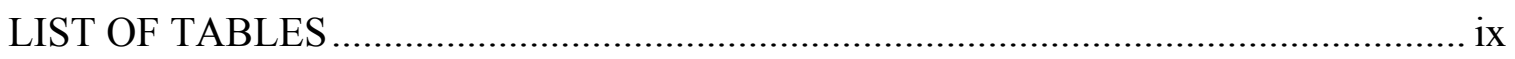

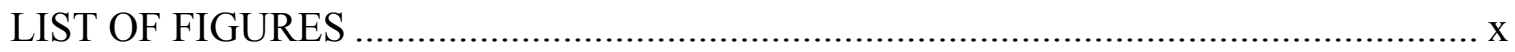

CHAPTER

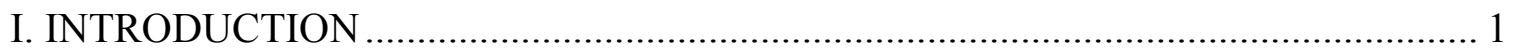

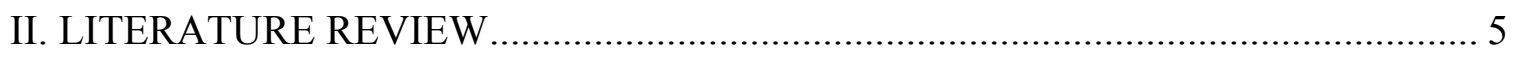

2.1 Molecular and Structural Charateristics of Liquid Crystalline Polymers ............... 5

2.2 Rheological Characteristics of Liquid Crystalline Polymers ................................ 10

2.2.1 Thermal and Deformation History Dependencies .......................................... 10

2.2.2 Low Viscosity in Nematic State ................................................................... 11

2.2.3 Three-Region Plot in Flow Curve............................................................ 12

2.2.4 Overshoots in Start-up Steady Shear Flow .................................................... 15

2.2.5 Negative Normal Stresses in Lyotropic Liquid Crystalline Polymers.............. 16

2.3 Rheological Theories of Liquid Crystalline Polymers........................................... 17

2.3.1 Leslie-Ericksen Theory ………………………......................................... 18

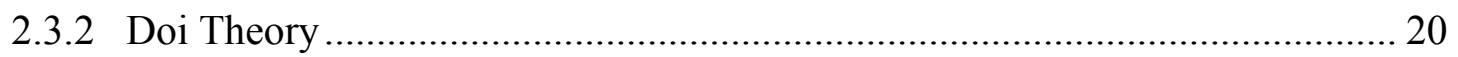

2.3.3 Leonov's Viscoelastic Nematodynamics ........................................................ 26

III. EXPERIMENTAL DATA AND MATERIALS CHARACTERISTICS OF LIQUID CRYSTALLINE POLYMERS USED IN SIMULATION ........................................ 30

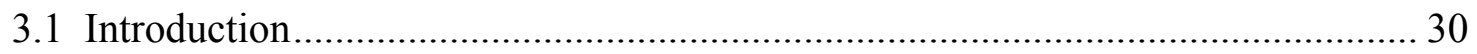


3.2 Molecular Characteristics and Rheological Properties of Titan and Zenite 6000

3.3 Molecular Characteristics and Rheological Properties of PSHQ9 and PI-14-5CN

IV. THEORETICAL MODEL OF SHEAR FLOW OF VISCOELASTIC DYNAMICS.

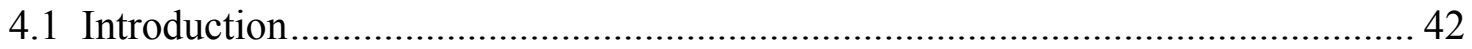

4.2 Thermodynamics and Constitutive Relations .................................................... 42

4.3 Closed Set of Maxwell Viscoelastic Nematodynamics ………………………...... 44

4.4 Weak Nematic Viscoelasticity in the Absence of Magnetic Field $(\underline{h}=0)$........... 47

4.5 Soft Deformation Modes in the Weak Maxwell Type Nematodynamics.............. 48

4.6 Equations of Weak Maxwell Type Nematodynamics in Simple Shearing............ 50

4.6.1 Evolution of Director Located in the $\left\{\mathrm{x}_{1}, \mathrm{x}_{2}\right\}$ Shear Plane ............................... 51

4.6.2 Formula for Director in Steady Shearing of Aligning Nematics ...................... 51

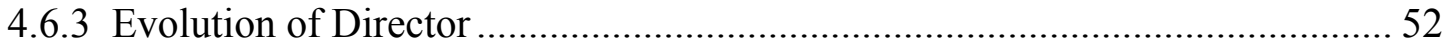

4.6.4 Unsteady Shearing of Aligning Viscoelastic Nematics ..................................... 54

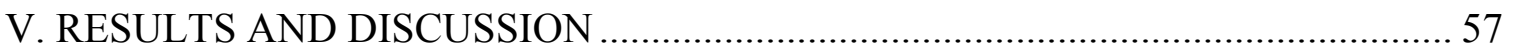

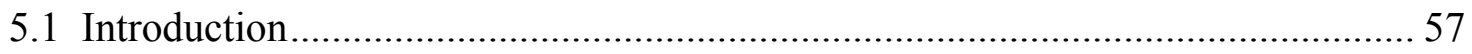

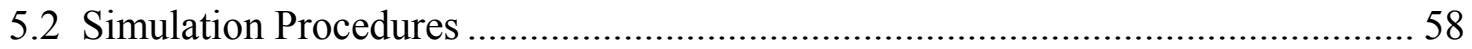

5.2.1 Determining Constitutive Parameters from Steady Shear Flows …………...... 58

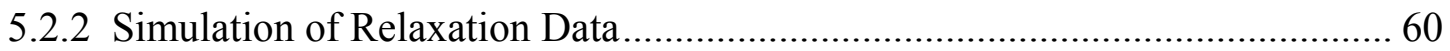

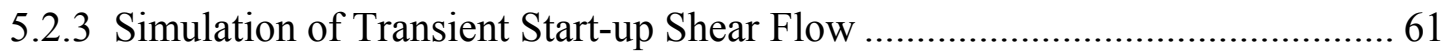

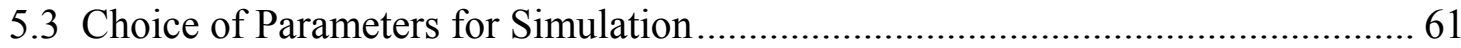

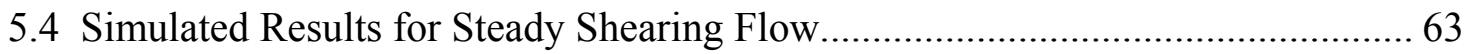

5.5 Simulated Results for Transient Start-up Shear Flow........................................ 68 
5.6 Simulated Results for Relaxation upon Cessation of Steady Flow.

5.7 On the Time-Temperature Superposition for Weakly Viscoelastic Nematodynamics.................................................... 79

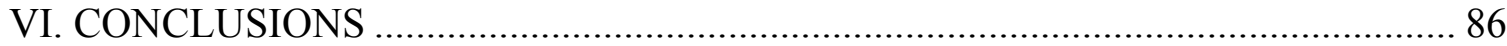

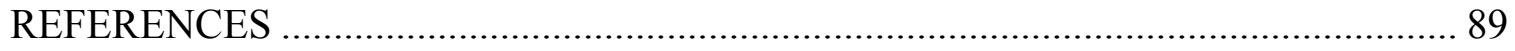

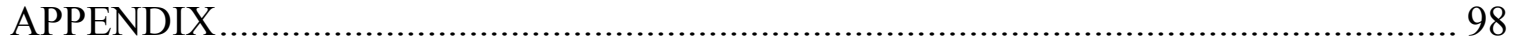




\section{LIST OF TABLES}

Table

Page

5.1 The constitutive parameters of simulating the experimental data of PSHQ9 at 130, 140 and $150{ }^{\circ} \mathrm{C}$

5.2 The constitutive parameters of simulating the experimental data of PI-14-5CN at 70, 80 and $90{ }^{\circ} \mathrm{C}$ 


\section{LIST OF FIGURES}

Figure

Page

2.1 Schematic representation of different types of liquid crystalline polymers.........6

2.2 Schematic describing the types of mesophases in liquid crystalline polymers........7

2.3 Shlieren texture of nematic liquid crystalline polymer [24] ................................ 9

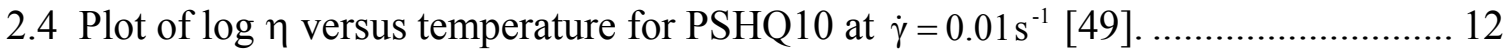

2.5 Effect of polymer concentration and the formation of a liquid-crystalline solution on the bulk viscosity of solutions of poly(chloro-1,4-phenylene/1,4-phenylene, 2,6naphthalamide) (70/30) in hexamethylphosphoramide-N-methylpyrrolidone (1:1,

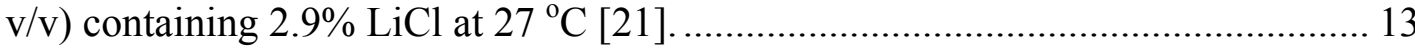

2.6 Schematic representation of three-region flow curve. ...................................... 14

2.7 Plots of (a) $\sigma^{+}(\dot{\gamma}, \mathrm{t})$ versus $\dot{\gamma} \mathrm{t}$ and $(\mathrm{b}) \mathrm{N}_{1}^{+}(\dot{\gamma}, \mathrm{t})$ versus $\dot{\gamma} \mathrm{t}$ during transient shear flow of PSHQ4-7CNCOOH at $130{ }^{\circ} \mathrm{C}$ at different shear rates $\left(\mathrm{s}^{-1}\right)$ : $(\bigcirc)$ 1.5, $(\triangle) 1.0,(\square)$

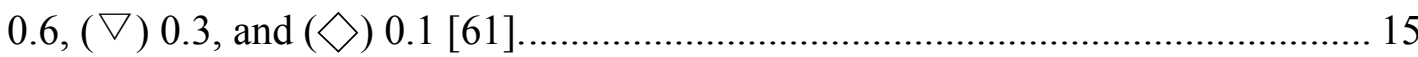

2.8 First normal stress difference versus shear rate for $14.1 \mathrm{wt}$ \% PBLG solution [25]. 17

3.1 Shear stress, normal stress and viscosity in steady flow of Titan at $340{ }^{\circ} \mathrm{C}$ [63] ...... 32

3.2 Transient shear flow and stress relaxation after cessation of steady shear flow for

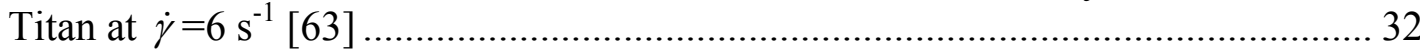

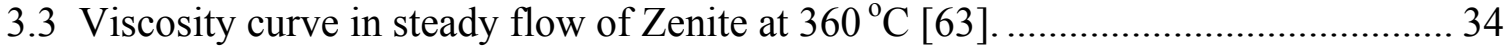

3.4 Time evolution of shear stress $\left(\sigma_{12}\right)$ at different shear rates $\left(1\right.$ and $\left.2 \mathrm{~s}^{-1}\right)$ in start up flow for Zenite 6000 at $360^{\circ} \mathrm{C}$ [63] ................................................................... 34

3.5 Plots of $\log \eta$ vs $\log \dot{\gamma}$ (open symbols) and $\log \mathrm{N}_{1}$ vs $\log \dot{\gamma}$ (filled symbols) for steady flow of PSHQ9 at different temperatures in the nematic region: $(\circ, \bullet) 130{ }^{\circ} \mathrm{C}$;

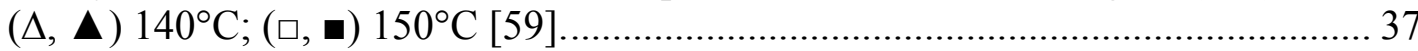


3.6 The time evolution of (a) $N_{1}^{+}(\dot{\gamma}, t) / N_{1}$ and (b) $\sigma^{+}(\dot{\gamma}, t) / \sigma$ for PSHQ9 at $140{ }^{\circ} \mathrm{C}$ in start-up shear flow at $\dot{\gamma}=1.0 \mathrm{~s}^{-1}[59]$.

3.7 Relaxation of normalized (a) shear stress, $\sigma^{-}(\dot{\gamma}, t) / \sigma_{0}$ and (b) first normal stress difference, $N_{1}^{-}(\dot{\gamma}, t) / N_{1,0}$ upon cessation of steady shear flow at different shear rates: (○) $\dot{\gamma}=1.0 \mathrm{~s}^{-1}$; ( $\left.\Delta\right) \dot{\gamma}=0.5 \mathrm{~s}^{-1}$; (口) $\dot{\gamma}=0.1 \mathrm{~s}^{-1}$ for PSHQ9 at $130^{\circ} \mathrm{C}$, where $\sigma_{0}$ and $N_{1,0}$ are the steady shear stress and first normal stress difference just prior to flow cessation, respectively [59].

3.8 Plots of $\log \eta$ vs $\log \dot{\gamma}$ (open symbols) and $\log \mathrm{N}_{1}$ vs $\log \dot{\gamma}$ (filled symbols) for PI$14-5 \mathrm{CN}$ in steady state at different temperatures in the nematic region: $(\circ, \bullet) 70{ }^{\circ} \mathrm{C}$;

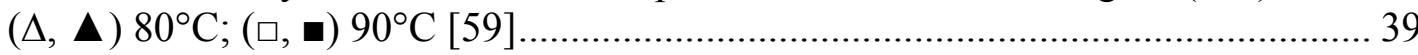

3.9 Time evolution of (a) $N_{1}^{+}(\dot{\gamma}, t) / N_{1}$ and (b) $\sigma^{+}(\dot{\gamma}, t) / \sigma$ for PI- $14-5 \mathrm{CN}$ at $70{ }^{\circ} \mathrm{C}$ in start-up shear flow at $\dot{\gamma}=1.0 \mathrm{~s}^{-1}$ [59].

3.10 Relaxation of normalized (a) shear stress, $\sigma^{-}(\dot{\gamma}, t) / \sigma_{0}$ and (b) first normal stress difference, $N_{1}^{-}(\dot{\gamma}, t) / N_{1,0}$ upon cessation of steady shear flow at different shear rates: (०) $\dot{\gamma}=1.0 \mathrm{~s}^{-1} ;(\Delta) \dot{\gamma}=0.5 \mathrm{~s}^{-1}$; (口) $\dot{\gamma}=0.1 \mathrm{~s}^{-1}$ for PI- $14-5 \mathrm{CN}$ at $70^{\circ} \mathrm{C}$, Where $\sigma_{0}$ and $N_{1,0}$ are the steady shear stress and first normal stress difference just prior to flow cessation, respectively [59]

5.1 Logarithmic plots of experimental data of viscosity $(\eta, \bullet)$, shear stress $\left(\sigma_{12}, \boldsymbol{\Delta}\right)$, and first normal stress difference $\left(\mathrm{N}_{1}, \boldsymbol{\square}\right)$ versus shear rate $(\dot{\gamma})$ for Titan at $340{ }^{\circ} \mathrm{C}$, with fitting curves of $\eta$ (solid line), $\sigma_{12}$ (short dash line), and $\mathrm{N}_{1}$ (long dash line).

5.2 Plots of viscosity $(\eta)$ versus shear rate $(\dot{\gamma})$ for Zenite 6000 at $360{ }^{\circ} \mathrm{C}$ : experimental data $(\bullet)$ and fitting curve (dashed line).

5.3 Plots of experimental data of $\log \eta$ vs $\log \dot{\gamma}$ (open symbols) and $\log \mathrm{N}_{1}$ vs $\log \dot{\gamma}$ (filled symbols) for PSHQ9 in steady flow at different temperatures in the nematic region: $(\circ, \bullet) 130^{\circ} \mathrm{C} ;(\Delta, \mathbf{\Delta}) 140^{\circ} \mathrm{C} ;(\square, \boldsymbol{\square}) 150^{\circ} \mathrm{C}$. The fitting curves for plots of $\log$ $\eta$ vs $\log \dot{\gamma}$ at different temperatures: (solid line) $130^{\circ} \mathrm{C}$, (long dashline) $140^{\circ} \mathrm{C}$, and (short dash line) $150^{\circ} \mathrm{C}$, as well as for $\log \mathrm{N}_{1}$ vs $\log \dot{\gamma}$ plots at different temperatures: (dotted line) $130^{\circ} \mathrm{C}$, (dash-dot line) $140^{\circ} \mathrm{C}$, and (dash-dot-dot line) $150^{\circ} \mathrm{C}$

5.4 Plots of experimental data of $\log \eta$ vs $\log \dot{\gamma}$ (open symbols) and $\log \mathrm{N}_{1}$ vs $\log \dot{\gamma}$ (filled symbols) for PI-14-5CN in steady state at different temperatures in the nematic region: $(\circ, \bullet) 70^{\circ} \mathrm{C} ;(\Delta, \mathbf{\Delta}) 80^{\circ} \mathrm{C} ;(\square, \boldsymbol{\bullet}) 90^{\circ} \mathrm{C}$. The fitting curves for $\log \eta$ vs $\log \dot{\gamma}$ plots at different temperatures: (solid line) $70^{\circ} \mathrm{C}$, (long dash line) $80^{\circ} \mathrm{C}$, 
and (short dash line) $90^{\circ} \mathrm{C}$, as well as for $\log \mathrm{N}_{1}$ vs $\log \dot{\gamma}$ plots at different temperatures: (dotted line) $70^{\circ} \mathrm{C}$, (dash-dot line) $80^{\circ} \mathrm{C}$, and (dash-dot-dot line) $90^{\circ} \mathrm{C}$.

5.5 Time evolution of normalized shear stress $\sigma^{+}(\dot{\gamma}, \mathrm{t}) / \sigma$ with strain $(\dot{\gamma} \mathrm{t})$ for Titan at 340 ${ }^{\circ} \mathrm{C}$ in start-up shear flow at $\dot{\gamma}=6 \mathrm{~s}^{-1}$ : experimental data $(\bullet)$ and fitting curve (dashed line). 70

5.6 Time evolution of normalized first normal stress difference $\mathrm{N}_{1}{ }^{+}(\dot{\gamma}, \mathrm{t}) / \mathrm{N}_{1}$ with strain ( $\dot{\gamma} \mathrm{t})$ for Titan at $340{ }^{\circ} \mathrm{C}$ in start-up shear flow at $\dot{\gamma}=6 \mathrm{~s}^{-1}$ : experimental data $(\bullet)$ and fitting curve (dashed line). 70

5.7 Time evolution of shear stress $(\sigma)$ with strain $(\dot{\gamma} \mathrm{t})$ for Zenite 6000 at $360{ }^{\circ} \mathrm{C}$ in startup shear flow at $\dot{\gamma}=2 \mathrm{~s}^{-1}$ : experimental data $(\bullet)$ and fitting curve (dashed line)...... 71

5.8 Time evolution of normalized shear stress $\sigma^{+}(\dot{\gamma}, \mathrm{t}) / \sigma$ with strain $(\dot{\gamma} \mathrm{t})$ for PSHQ9 at $130{ }^{\circ} \mathrm{C}$ in start-up shear flow at $\dot{\gamma}=1 \mathrm{~s}^{-1}$ : experimental data $(\bullet)$ and fitting curve

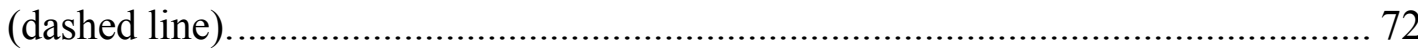

5.9 Time evolution of normalized first normal stress difference $\mathrm{N}_{1}{ }^{+}(\dot{\gamma}, \mathrm{t}) / \mathrm{N}_{1}$ with strain

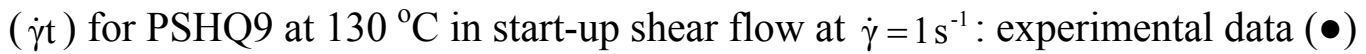
and fitting curve (dashed line).

5.10 Time evolution of normalized shear stress $\sigma^{+}(\dot{\gamma}, \mathrm{t}) / \sigma$ with strain $(\dot{\gamma} \mathrm{t})$ for PI-14-5CN at $70{ }^{\circ} \mathrm{C}$ in start-up shear flow at $\dot{\gamma}=1 \mathrm{~s}^{-1}$ : experimental data $(\bullet)$ and fitting curve (dashed line)

5.11 Time evolution of normalized first normal stress difference $\mathrm{N}_{1}^{+}(\dot{\gamma}, \mathrm{t}) / \mathrm{N}_{1}$ with strain ( $\dot{\gamma}$ t) for PI-14-5CN at $70{ }^{\circ} \mathrm{C}$ in start-up shear flow at $\dot{\gamma}=1 \mathrm{~s}^{-1}$ : experimental data (•) and fitting curve (dashed line).

5.12 Relaxation of shear stress $\left(\sigma_{12}\right)$ for Titan at $340{ }^{\circ} \mathrm{C}$ upon cessation of steady flow at shear rate $\dot{\gamma}=6 \mathrm{~s}^{-1}$ : experimental data $(\bullet)$ and fitting curve (dashed line). 76

5.13 Relaxation of first normal stress difference $\left(\mathrm{N}_{1}\right)$ for Titan at $340{ }^{\circ} \mathrm{C}$ upon cessation of steady flow at shear rate $\dot{\gamma}=6 \mathrm{~s}^{-1}$ : experimental data (•) and fitting curve (dashed line). 76

5.14 Relaxation of normalized (a) shear stress, $\sigma^{-}(\dot{\gamma}, t) / \sigma_{0}$ and (b) first normal stress difference, $N_{1}^{-}(\dot{\gamma}, t) / N_{1,0}$ upon cessation of steady shear flow at $\dot{\gamma}=0.5 \mathrm{~s}^{-1}$ for PSHQ9 at $130^{\circ} \mathrm{C}:(\bullet)$ experimental data and (dashed line) fitting curve. Here $\sigma_{0}$ 
and $N_{1,0}$ are the steady shear stress and first normal stress difference just prior to flow cessation, respectively.

5.15 Relaxation of normalized (a) shear stress, $\sigma^{-}(\dot{\gamma}, t) / \sigma_{0}$ and (b) first normal stress difference, $N_{1}^{-}(\dot{\gamma}, t) / N_{1,0}$ upon cessation of steady shear flow at $\dot{\gamma}=1 \mathrm{~s}^{-1}$ for PSHQ9 at $130^{\circ} \mathrm{C}:(\bullet)$ experimental data and (dashed line) fitting curve. Here $\sigma_{0}$ and $N_{1,0}$ are the steady shear stress and first normal stress difference just prior to flow cessation, respectively.

5.16 Relaxation of normalized (a) shear stress, $\sigma^{-}(\dot{\gamma}, t) / \sigma_{0}$ and (b) first normal stress difference, $N_{1}^{-}(\dot{\gamma}, t) / N_{1,0}$ upon cessation of steady shear flow at $\dot{\gamma}=0.5 \mathrm{~s}^{-1}$ for PI$14-5 \mathrm{CN}$ at $70^{\circ} \mathrm{C}:(\bullet)$ experimental data and (dashed line) fitting curve. Here $\sigma_{0}$ and $N_{1,0}$ are the steady-state shear stress and first normal stress difference just prior to flow cessation, respectively.

5.17 Relaxation of normalized (a) shear stress, $\sigma^{-}(\dot{\gamma}, t) / \sigma_{0}$ and (b) first normal stress difference, $N_{1}^{-}(\dot{\gamma}, t) / N_{1,0}$ upon cessation of steady shear flow at $\dot{\gamma}=1 \mathrm{~s}^{-1}$ for PI$14-5 \mathrm{CN}$ at $70^{\circ} \mathrm{C}:(\bullet)$ experimental data and (dashed line) fitting curve. Here $\sigma_{0}$ and $N_{1,0}$ are the steady-state shear stress and first normal stress difference just prior to flow cessation, respectively.

5.18 Master curves of experimental data (a) $\log \hat{\eta}=\eta / \eta_{0}$ vs $\log \hat{\dot{\gamma}}=\theta_{0} \dot{\gamma} \quad$ (open symbols) and (b) $\log \hat{N}_{1}=N_{1} \theta_{0} / \eta_{0}$ vs $\log \hat{\dot{\gamma}}=\theta_{0} \dot{\gamma}$ (filled symbols) for PSHQ9 in steady flow at different temperatures in the nematic region: $(\circ, \bullet) 130{ }^{\circ} \mathrm{C} ;(\Delta, \boldsymbol{\Delta}) 140^{\circ} \mathrm{C} ;(\square, \boldsymbol{\square})$ $150^{\circ} \mathrm{C}$. Corresponding fitted theoretical master curves at different temperatures are shown by solid lines.

5.19 Master curves and experimental data for (a) $\log \hat{\eta}=\eta / \eta_{0}$ vs $\log \hat{\dot{\gamma}}=\theta_{0} \dot{\gamma} \quad$ (open symbols) and (b) $\log \hat{N}_{1}=N_{1} \theta_{0} / \eta_{0}$ vs $\log \hat{\dot{\gamma}}=\theta_{0} \dot{\gamma}$ (filled symbols) for PI-14-5CN in steady shearing at different temperatures in the nematic region: $(\circ, \bullet) 70^{\circ} \mathrm{C} ;(\Delta, \boldsymbol{\Delta})$ $80{ }^{\circ} \mathrm{C} ;(\square, \boldsymbol{\square}) 90^{\circ} \mathrm{C}$. Corresponding fitted theoretical master curves at different temperatures are shown by solid lines. 


\section{CHAPTER I}

\section{INTRODUCTION}

The quest for lightweight materials with great strength and stiffness has led to the synthesis of liquid crystalline polymers (LCP's) and occurrence of novel processes and theories to predict and control the LCP structures in final products. To date, liquid crystalline polymers have found a variety of applications such as high-strength plastic fibers, bullet-proof garments, front panels of computers, cellular phones, electronic diaries, portable televisions, printed circuit boards, etc [1]. Typically LCP's have outstanding mechanical properties at high temperatures, excellent chemical resistance, inherent flame retardancy, heat aging resistance, low viscosity, and good weatherability. It makes LCP's ideal candidates for high-performance applications [2].

Specific polymers display LCP properties in a certain intermediate state between the solids and liquids, which is called mesophase, with the combined properties of both the crystalline and liquid state [3]. Thus, the orientational order among the molecules makes the material anisotropic and "crystalline," while the lack of strong positional order allows the material to flow like ordinary fluids. LCP's whose phase transition into the liquid crystalline phase occurs under change in temperature are called "thermotropic", while a variety of LCP's which exhibit phase transitions by changing the polymer concentration in a solvent (as well as temperature) are called "lyotropic" liquid crystals. The unique feature of mesophase is that it is described by a positional order parameter, 
because it is geometrically anisotropic in space. The molecules in LCP's are arranged in an orderly manner, and there are three different types of ordered structures in LCP's, nematic, cholesteric, and smectic.

The nematic mesophase, where the molecules possess a long-range orientational order but only a short-range positional order $[4,5]$ can be met more often, because it has low ordered structure between other LCP types. Currently, the polymer nematics include liquid crystalline polymers and liquid crystalline elastomers (LCE's) [6]. The action of external field or flow causes the orientation of mesogens of LCP's or LCE's, which induces uniaxial anisotropy, with an additional degree of freedom, internal rotations [7]. In certain cases, LCP's or LCE's possesses partial flexibility, which displays macroscopically as anisotropic viscoelasticity and thus is important for dynamics of these systems.

de Gennes and Prost [5] first developed the concept of nematodynamics, which is referred to a specific set of problems for deformation and flow of the nematic systems under stress and external (magnetic and electrical) fields that can be solved or analyzed using specific macroscopic field equations. The research on polymer nematics has attracted long-standing academic and industrial interests for about three decades, but only few of them have been in practical use, which is due to the lack of knowledge of the complicated behavior of these systems. Therefore, nematodynamics studies are imperative for stimulating the progress in processing of polymer nematics and predicting the properties of post-processed products.

Two types of theories - continuum and molecular - have been currently proposed to attack the problem of modeling LCP properties. The continuum theories are developed 
to establish a general framework with minimum assumptions about the molecular structures of LCP's, whereas the molecular approaches employ very specific assumptions of structure of these polymers, but unlike the continuum approaches, operate with few molecular parameters. It should be pointed out that these theories, though well separated, are not contradictory but supplemental.

Notably, large fluctuations are typical features for the nematic systems [8, 9]. Therefore using attractively few-parametric mean-field molecular approaches leads to poor predictions of experimental data for LCP's [5]. Consequently, nematic studies usually resort to continuum approaches based on fundamental principles of thermodynamics and symmetry. The beauty of continuum approach is that it can be simultaneously applied to the molecular nematics of both different types as well as to the non-yielding suspensions. Unfortunately, general nematodynamic theories are multiparametric. For example, general Leslie-Ericksen-Parodi (LEP) continuum theory of low molecular weight (LMW) LC's contains five constitutive parameters [10]. Similarly, the monodomain de Gennes's potential for describling general weakly elastic behavior of LCE's has five parameters [11]. Thus, it is expected that even in easy theoretical schemes, the continuum approach to polymer (viscoelastic) nematodynamics should involve at least ten constitutive parameters. This gives rise to a pessimistic view that the continuum theories of these systems are intractable.

It should be mentioned that neither molecular nor continuum theory existed till now for describing complicated properties of thermotropic LCP, although many experimental data for this type of LCP have been accumulated. Recently, Leonov [12-14] has developed a new continuum theory of weakly nonlinear viscoelastic nematodynamics 
aimed in particular, to close this gap between theory and experiments. New techniques used in this theory were very helpful in several derivations to present the theory in a simple form. The assumption of small transient (elastic) strains and transient relative rotations, employed in the theory, is appropriate for most LCP's, which usually display a small macromolecular flexibility. This theory nontrivially combines the LEP approach for viscous nematodynamics and that for weak nematic solids, utilizing a specific viscoelastic and nematic kinematics. It should be mentioned that this theory is mainly suitable for monodomain molecular nematics. Nevertheless, the effects of Frank (orientation) elasticity are also included in the viscoelastic nematodynamics theory to describe the multi-domain effects in flows of LCP's near the equilibrium. In the absence of external fields and neglecting the Frank elasticity, the simplified theory can employ less parametric description for LCP's flows. Additional decrease in number of parameters happens in cases of existence of viscoelastic soft/semi-soft nematic modes.

This study aims at testing the continuum theory of weak viscoelastic nematodynamics [12-14]. The objective is to analytically and numerically investigate steady and unsteady basic shear flows of polymer nematics with possible aligning and tumbling effects, and compare the theoretical predictions with published experimental data. Hopefully, this research will create a reliable and fundamental continuum framework for more detailed theories for polymer nematics. It is also expected that the results of these fundamental studies will significantly contribute in developing robust and reliable dynamic and flow models that will be used in analyses of processing and post processing properties of polymer nematic systems. The current lack of such models prevents the progress in processing of LCP's and LCE's. 


\section{CHAPTER II}

\section{LITERATURE REVIEW}

\subsection{Molecular and Structural Charateristics of Liquid Crystalline Polymers}

The beauty of liquid crystalline phase and unique properties of liquid crystalline polymers (LCP's) come from their ordered, yet liquid, state, which are finally predetermined by their chemical structures. To the best of our knowledge, LCP's are largely derived from monomeric liquid crystals, which form liquid crystalline phase arising from rigid units such as biphenyl or terphenyl groups with oblate or prolate shape and aspect ratios of three or more [15]. The rigid or semi-rigid units in LCP's are called mesogens. Based on molecular architecture, LCP's are divided into three types [16] as shown schematically in Figure 2.1: (i) main-chain LCP's, (ii) side-chain LCP's, and (iii) combined main-chain and side-chain LCP's. In main-chain LCP's, the mesogen is located in the polymer backbone. Some bulky pendent groups, molecular kinks or flexible spacers are usually introduced into the polymer backbone to suppress crystallinity and reduce the clearing temperature. In the case of side-chain LCP's, the mesogen is attached to the polymer backbone as a pendant group directly or through a flexible spacer. Futher on, the combined main-chain and side-chain LCP's have mesogens in both polymer backbone and side chains. 
(i) Main Chain LCPs

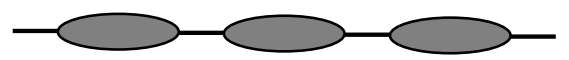

(a) Polymerized by direct connection

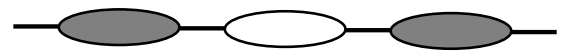

(b) Random copolymers

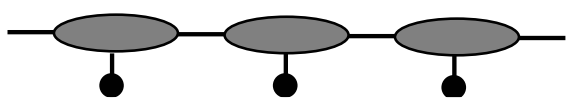

(c) Polymers with bulky pendent groups

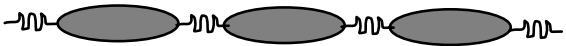

(d) Polymers with flexible spacers

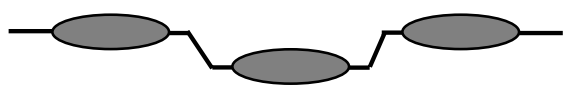

(e) Polymers with kinks (ii) Side Chain LCPs

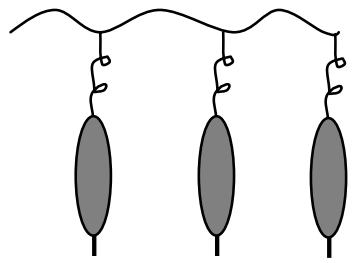

(a) Mesogen perpendicular to main chain

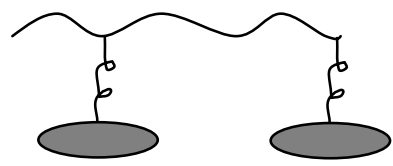

(b) Mesogen paralel to main chain

(iii) Combined Main Chain and Side Chain LCPs

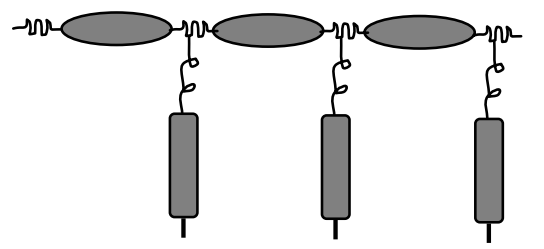

(a) Side chain attached to flexible spacer

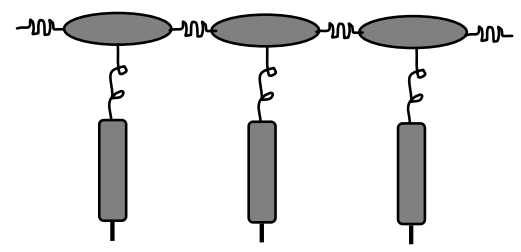

(b) Side chain attached to mesogen

Figure 2.1 Schematic representation of different types of liquid crystalline polymers 
LCP's can also exhibit multiple-liquid crystalline phases, depending on temprature or concentration. The molecules of LCP's posses at least some orientational order in arrangement, but they are lacky of three-dimensional positional order of solid crystals. The order structure of an LCP phase can be nematic phase that possesses a long-range orientational order but only a short-range positional order, cholestoric phase that is twisted periodically about an axis perpendicular to the director, or smectic phase that has stratified structure with a variety of molecular arrangements within each layer (see Figure 2.2). It should be pointed out that the tendency to form a nemtic state is controlled mainly by the axial ratio, $b_{K} / d$, where $b_{K}$ is the Kuhn length, which is twice the persistence length $\lambda_{p}$, and $\mathrm{d}$, the effective diameter of the molecule [17]. Thus, theomotropic LCP's

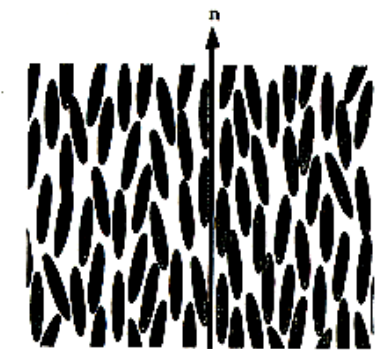

(a) Nematic

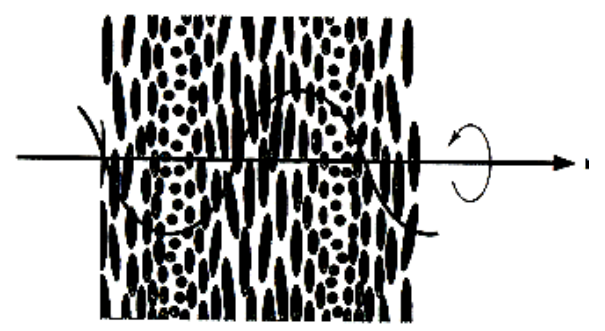

(b) Cholesteric

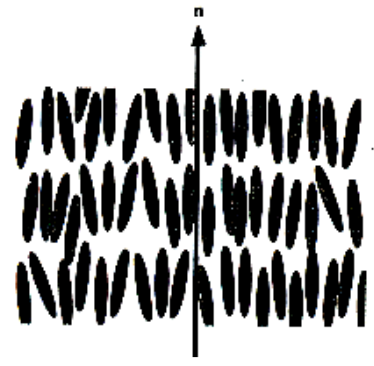

(c) Smectic A

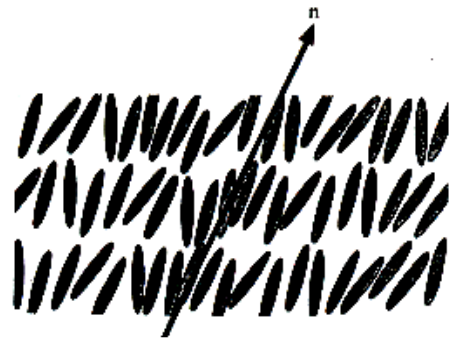

(d) Smectic C

Figure 2.2 Schematic describing the types of mesophases in liquid crystalline polymers. 
are usually composed of semi-flexible molecules whose persistence length $\lambda_{p}$ is much shorter than the molecular length $\mathrm{L}$, i.e. $\mathrm{L}>>\lambda_{p}[18]$.

Liquid crystalline polymers (LCP's) may also be divided into two subgroups: (1) lyotropic LCP's, formed by mixing LCP's with a solvent, and (2) thermotropic LCP's, formed by heating or cooling. Lyotropic LCP's such as poly(p-phenylene terephthalamide) (PPTA), poly(p-benzamide) (PBA), poly( $\gamma$-benzyl L-glutamate) (PBLG), and hydroxypropylcellulose (HPC) have a high degree of chain rigidity, giving rise to the high melting temperature of these polymers $[19,20]$. Therefore, these polymers must be processed in the solution, for example, Kelvar $^{\mathrm{TM}}$ fibers are spun using solution spinning techniques [21]. Of particular interest here is the fact that these polymers form anisotropic solutions in solvents, which is believed to be the key factor in achieving the unusually strong mechanical properties of Kelvar fiber. On the other hand, thermotropic LCP's are very attractive to industry from the processing point of view, because there is no problem of solvent recovery. One approach is to incorporate mesogenic groups into the flexible main chain, for example, polyethylene terephthalate (PET) has successfully been utilized to modify with p-hydroxybenzoic acid (HBA), which exhibits liquidcrystalline structure when the concentration of HBA in the copolyester is higher than 40 mol\% [22]. Other thermotropic LCP's, such as copolyesters of 4,4'-biphenol, terephthalic acid, and HBA, as well as copolyesters of HBA and 6-hydroxy-2-naphthoic acid (HNA) have also been commercialized. Lenz et al. [23] synthesized a series of thermally stable, semiflexible thermotropic LCPs, like PSHQ10 having the chemical structure: 


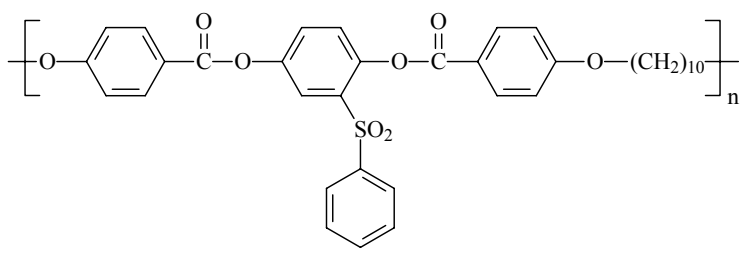

Schlieren texture (a characteristic threadlike structure) shown in Figure 2.3 is often observed under polarized optical microscope for nematic thermotropic LCP's [24]. Other kinds of textures for defect are also possible. Nematics frequently contain defects named as disclinations (see the dark line in Figure 2.3), where the director abruptly changes orientation. The most common of these are the line disclination, although point-wise disclinations are also observed [5]. Owing to defects and distortions, the director is nonuniformly distributed in space; the sample therefore has a polydomain structure. Thus, it is extremely difficult to release LCP's from defects, and even when it is possible, the result is extremely fragile, as a shear flow will soon destroy the monodomain. To date, there is no theory that takes into account the effect of disclination. This is because of complexity of dependency of temperature and stress.

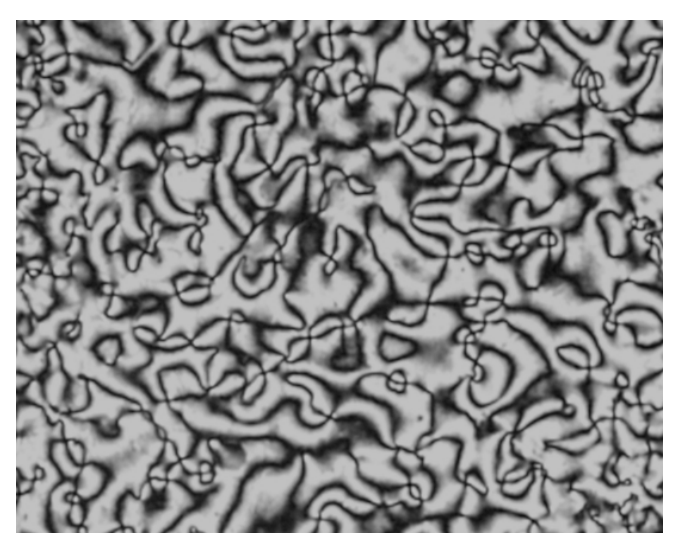

Figure 2.3 Shlieren texture of nematic liquid crystalline polymer [24]. 


\subsection{Rheological Characteristics of Liquid Crystalline Polymers}

The rigid chemical elements in LCP's structure result in a variety of particular rheological properties, which are quite different from those of polymers with flexible chains. During the past three decades much attention has been attracted to the experimental investigations of rheological behavior of LCP's. Some investigators reported the rheological behavior of lyotropic solutions of poly( $\gamma$-benzyl-L-glutamate $)$ (PBLG) [25-34], hydroxypropylcellulose (HPC) [35-39], and other rodlike stiff polymers [40-42], while other researchers reported the rheological behavior of thermotropic mainchain LCP's [43-53] and still others reported the rheological behavior of thermotropic side-chain LCP's [54-60] as well as combined main-chain and side-chain LCP's [61]. We present below several unusual rheological properties observed for liquid crystalline polymers.

\subsubsection{Thermal and Deformation History Dependencies}

As different from flexible homopolymers, the rheological behavior of LCP's is highly dependent on thermal and deformation histories $[49,50,62]$. This is primarily due to the rather complexed structure of LCP's whose morphology depends on previous deformation, and also varies with the change in temperature. Therefore, only under the identical initial conditions (i.e. morphologies) the reproducible rheological data can be achieved; otherwise, it would not be possible for one to separate the effect of test temperature and the effect of thermal history from the overall rheological response. One strategy to control the initial conditions is to heat the sample above the clearing temperature of LCP's in the isotropic state, and then cool to the measured temperature before making rheological measurements [50]. This strategy can only be achieved when 
the clearing temperature of LCP's is far below their respective thermal degradation temperature. Unfortunately, the clearing temperatures of most commercial LCP's today are close to or higher than their degradation temperatures. Consequently, another strategy was proposed by Belatreche [63] through pre-shearing the sample at the preset measured temperature, and then relaxing before conducting rheological measurements. In such a way, the rheological behaviors of Titan and Zenite 6000 were successfully investigated.

\subsubsection{Low Viscosity in Nematic State}

The lower viscosity exhibited by liquid crystalline polymer in the nematic phase is one of their attractive features. Although LCP's generally have a fairly rigid chain structure, which forms the basis of their exceptional properties in the solid state, they can be processed in the nematic phase relatively easily. It should be pointed out that the shear viscosities are higher in the isotropic phase, despite its higher temperature, than in the anisotropic phase $[25,26,45]$. The temperatue $(T)$ dependence of viscosity $(\eta)$ of PSHQ10 shown in Figure 2.4 [49], is different in three regions: (a) in the nematic region, within the $T$ region less than $160{ }^{\circ} \mathrm{C}$ where $\eta$ decreases with increasing $T$; (b) in the biphase region, where $160{ }^{\circ} \mathrm{C}<\mathrm{T}<175^{\circ} \mathrm{C}$ with $\eta$ increases with increasing $T$, which is due to the polydisperse nature of PSHQ10, where some molecules may have already transformed into an isotropic state at the temperatures below $175{ }^{\circ} \mathrm{C}$ with biphase formation; and (c) in the isotropic region, where $\mathrm{T}>175^{\circ} \mathrm{C}$ and $\eta$ decreases with increasing $T$, following the Arrhenius rule. 


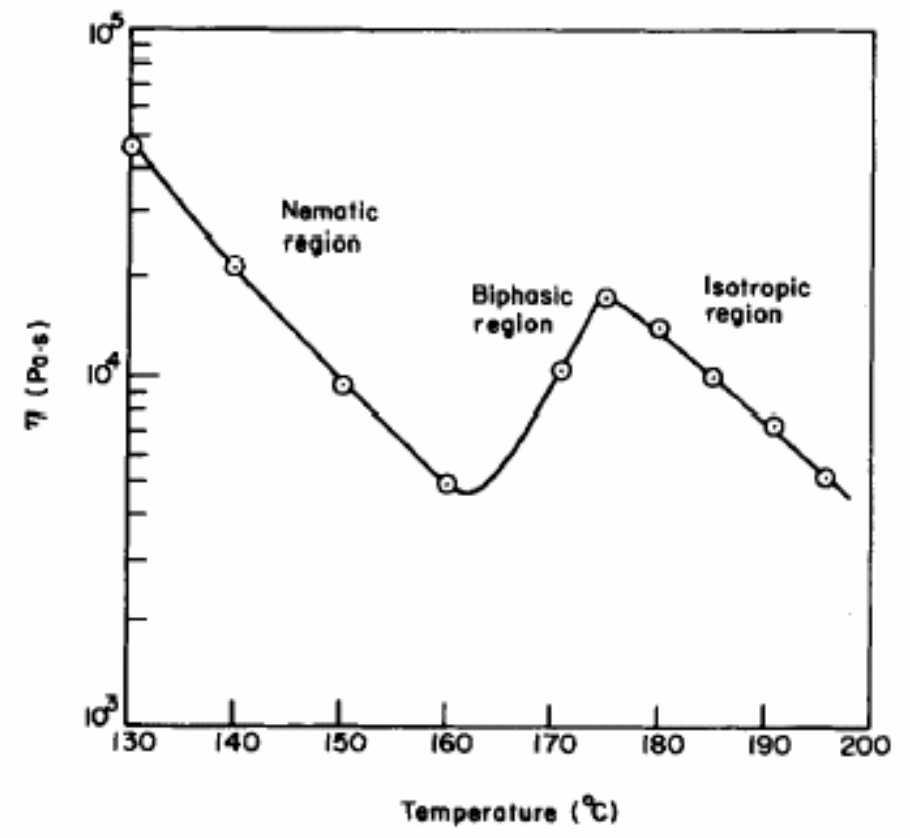

Figure 2.4 Plot of $\log \eta$ versus temperature for PSHQ10 at $\dot{\gamma}=0.01 \mathrm{~s}^{-1}$ [49].

A similar behavior is also observed in the lyotropic case [21] where the bulk viscosity suddenly changes with increasing polymer concentration at the point where the liquid-crystalline order begins (see Figure 2.5). At the left side of Figure 2.5, the viscosity of solution rises in the way common for isotropic solutions. At a critical point, the system is capable to accommodate more polymer only if an ordered, liquid-crystalline phase can be formed. When this occurs it is followed by decreasing viscosity and increasing amount of ordered phase. It then keeps decreasing well within the nematic phase, to finally increase again at even larger concentration.

\subsubsection{Three-Region Plot in Flow Curve}

As distinguished from ordinary polymers, the curve of viscosity versus shear rate of LCP's has the typical shape of three regions shown in Figure 2.6. This unusual behavior 


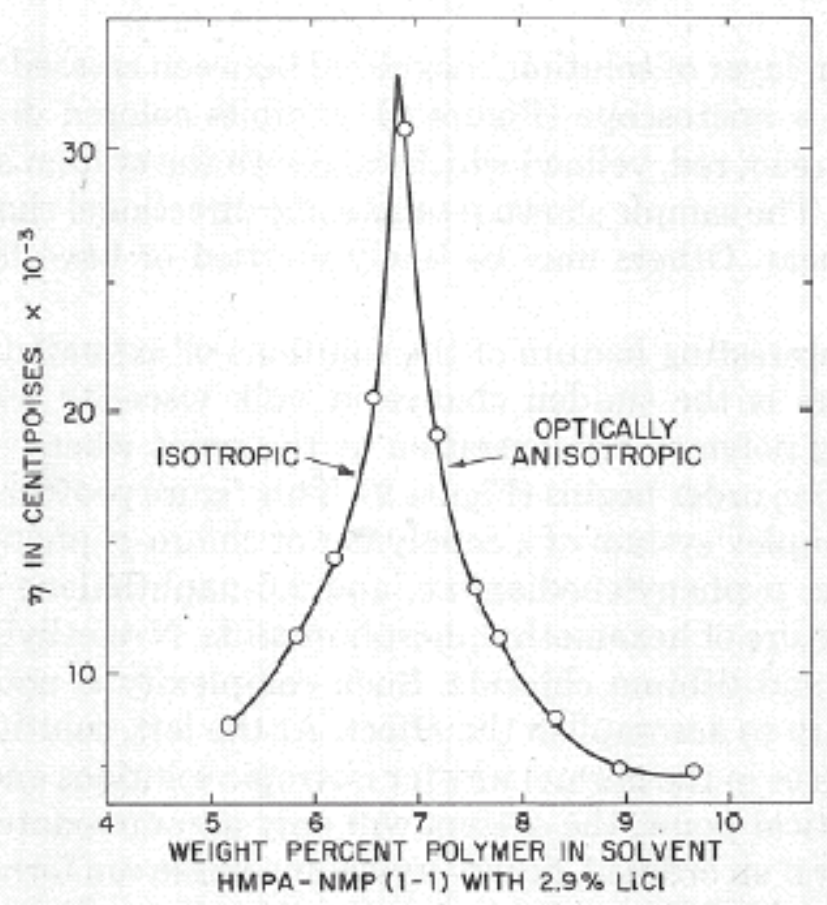

Figure 2.5 Effect of polymer concentration and the formation of a liquid-crystalline solution on the bulk viscosity of solutions of poly(chloro-1,4-phenylene/1,4-phenylene, 2,6-naphthalamide) (70/30) in hexamethylphosphoramide-N-methylpyrrolidone (1:1, $\mathrm{v} / \mathrm{v}$ ) containing $2.9 \% \mathrm{LiCl}$ at $27{ }^{\circ} \mathrm{C}[21]$.

was first reported for lyotropic LCP solution by Onogi [64], and was believed to be related to the variations of the morphological state of the fluid in different regions. In region I with low shear rate, nematic LCP is shear thinning; i.e., the viscosity decreases with increasing shear rate. Ordinary polymers with flexible chains do not display the region I behavior; i.e. their viscosity always approaches a constant value as the shear rate tends to zero. The region I behavior happens for lyotropic LCP because at very low shear rate, the texture of nematic phase constists of many small domains, in which the local orientations of the director vary from one domain to another. Under this circumstance, each small domain may act as if it is a discrete particle suspended in a very low viscosity 
fluid (nematic solvent). Thus, at extremely low shear rate, the polydomain hardly deforms. The structural evolution of such a defect 'suspension' would generate the shear thinning behavior at low shear rates [65].

There then follows a region where the viscosity is almost constant with shear rate (Newtonian behavior), which is referred to the region II. As the shear rate increases, the increased effect of coalescence results in reducing the number of small domains, while the local orientation of the director begins to align in the flow direction. Finally, at even larger shear rates, the material exhibits shear thinning again in region III. Throughout region I and a part of region II, the polydomain structure is preserved in spite of the flow: no significant net orientation of the director is induced by the flow process. When the intensity of shear flow becomes sufficiently high, the local orientation of director in separate small domains are aligned perfectly along the flow direction, as if the various domains had coalesced into a single one. Burghardt et al. [66] used birefrignece to directly observe the structural changes during shear flow of lyotropic LCP solution.

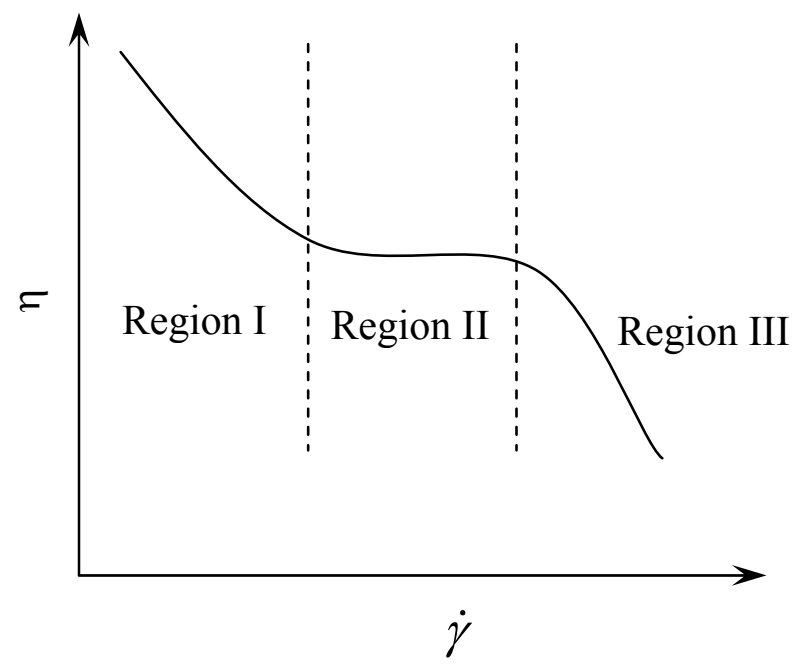

Figure 2.6 Schematic representation of three-region flow curve. 


\subsubsection{Overshoots in Start-up Steady Shear Flow}

Unlike flexible homopolymers, liquid crystalline polymers often show multiple overshoots of shear stress $\left(\sigma_{12}\right)$ or first normal stress difference $\left(\mathrm{N}_{1}\right)$ in the transient shear flow, which is one of the unique rheological characteristics of LCPs. A typical example of overshoots during transient shear flow of a combined main-chain \& side chain LCP, PSHQ4-7CNCOOH at $130{ }^{\circ} \mathrm{C}$ is given in Figure 2.7 [61], where one can observe that the values of overshoots of $\sigma^{+}(\dot{\gamma}, \mathrm{t})$ and $\mathrm{N}_{1}^{+}(\dot{\gamma}, \mathrm{t})$ increase with the increased shear rate. The large overshoots of LCP's are attributed to the existence of polydomains in the

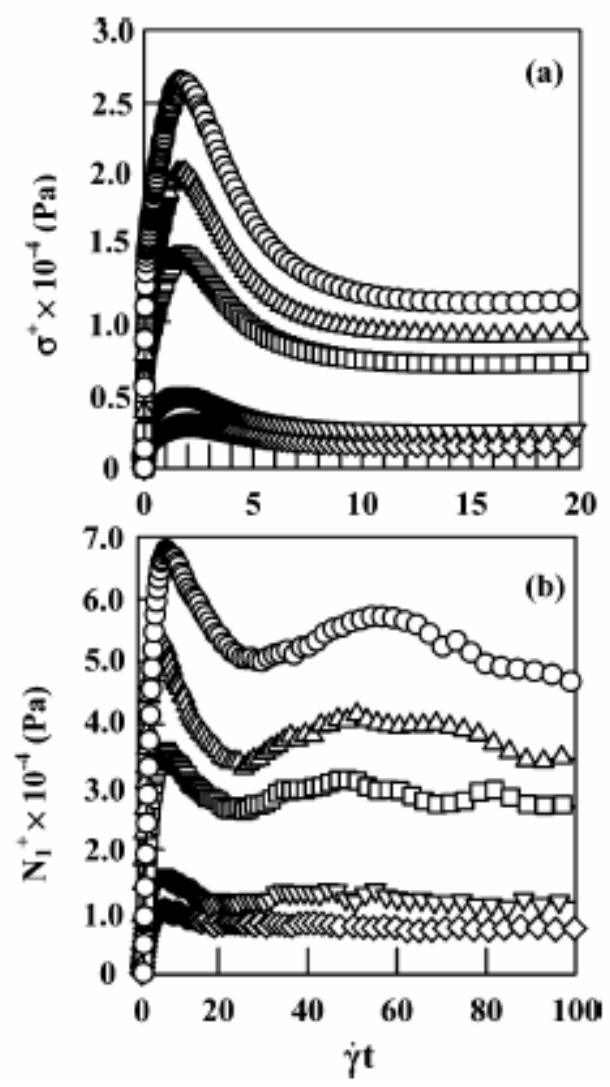

Figure 2.7 Plots of (a) $\sigma^{+}(\dot{\gamma}, \mathrm{t})$ versus $\dot{\gamma}$ and (b) $\mathrm{N}_{1}^{+}(\dot{\gamma}, \mathrm{t})$ versus $\dot{\gamma}$ t during transient shear flow of PSHQ4-7CNCOOH at $130{ }^{\circ} \mathrm{C}$ at different shear rates $\left(\mathrm{s}^{-1}\right):(\bigcirc) 1.5,(\triangle) 1.0,(\square)$ $0.6,(\nabla) 0.3$, and $(\diamond) 0.1[61]$. 
nematic state. When subjected to a sudden shear flow, the grain boundaries of polydomains disappear, tending to give rise to monodomains where all directors are aligned more or less in the same direction. The theorectical interpretation of this unusal transient shear flow behavior of LCPs has been independently considered by Marrucci and Maffettone [67] and Larson [69], which will be discussed later.

\subsubsection{Negative Normal Stresses in Lyotropic Liquid Crystalline Polymers}

Kiss and Porter [25-27] first reported negative values of normal stress $\left(\mathrm{N}_{1}\right)$ in steady shear flow over a certain range of shear rates for lyotropic solutions of PBLG in m-cresol, as shown in Figure 2.8. Since then, other experimental results for lyotropic solution of HPC $[33,36,39]$ also confirmed the observation of negative $\mathrm{N}_{1}$. It should be mentioned that to the best of our knowledge, the phenomenon of negative $\mathrm{N}_{1}$ has never been observed for thermotropic LCP's.

Marrucci and Maffettone [72] and Larson [69] independently theoretically elucidated the origin of such unusual rheological behavior of some lyotropic solutions. Specifically, using the Maier-Saupe potential, Marrucci and Maffettone [72] conducted a two-dimensional analysis of shear flow of the nematic monodomains of lyotropic solutions of rodlike polymers and obtained analytical expressions relating the sign of $\mathrm{N}_{1}$ to director tumbling. Finally, they made a conclusion that the occurrence of a negative $\mathrm{N}_{1}$ is associated with a flow transition between director tumbling and flow-aligning behavior. On the other hand, using Doi equation together with the Onsager potential, Larson [69] independently conducted a three-dimensional analysis of shear flow of the nematic monodomains of lyotropic solution of rodlike polymers, and solved numerically the system equations by expansion in spherical harmonic functions. He concluded that 


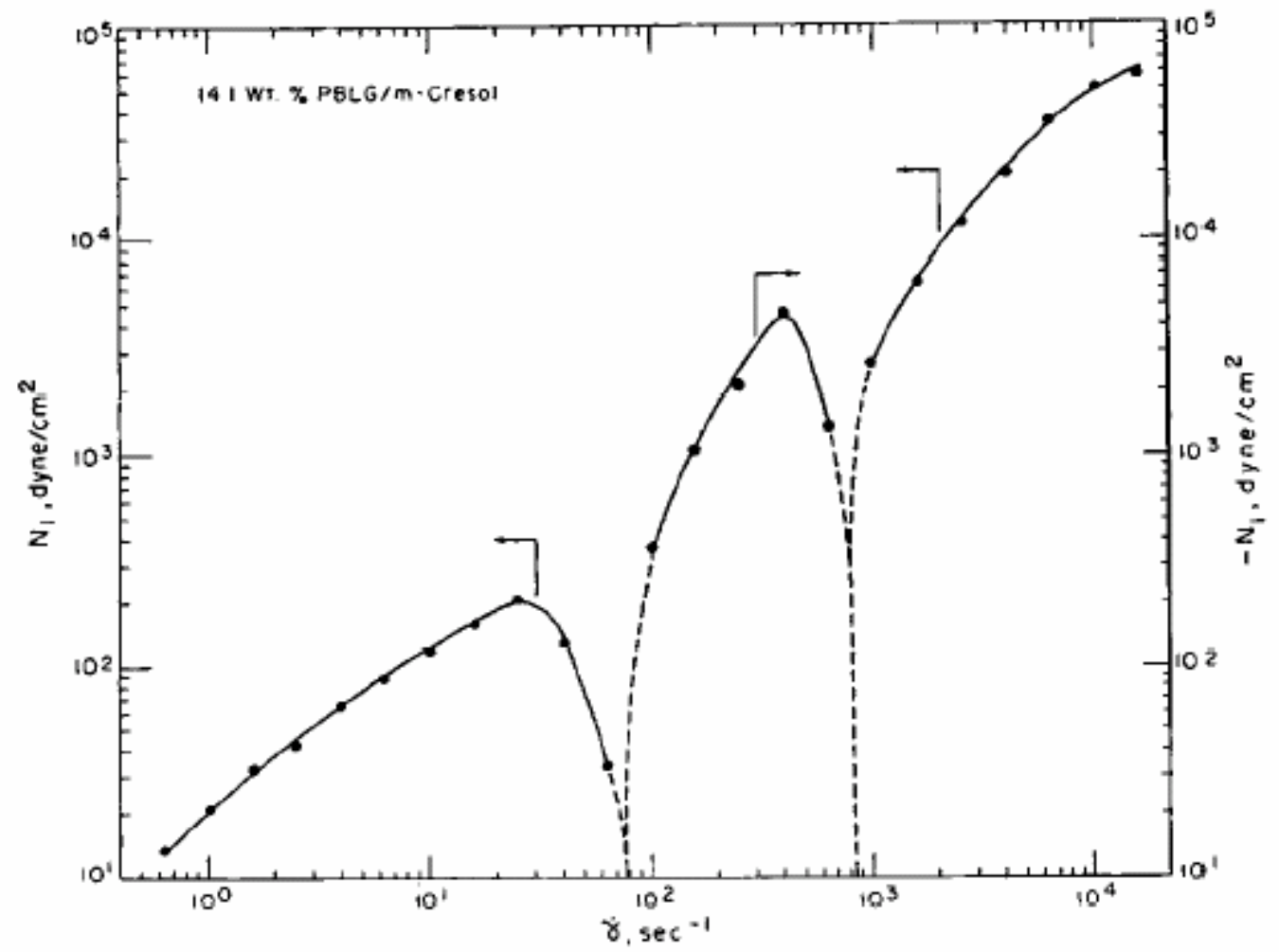

Figure 2.8 First normal stress difference versus shear rate for $14.1 \mathrm{wt}$ \% $\%$ PBLG solution [25].

negative $\mathrm{N}_{1}$ occurs in the "wagging" regime representing the transition from the tumbling to the nontumbling flow (flow-aligning) regime. The three-dimensional Larson analysis confirms the two-dimensional Marrucci-Maffettone analysis, indicating that negative $\mathrm{N}_{1}$ is associated with a flow transition between director tumbling and flow-aligning behavior.

\subsection{Rheological Theories of Liquid Crystalline Polymers}

During the last three decades, there has been a significant advance in both theoretical [5,7,69-70,73-82] and experimental [25-61] studies of nematic liquid crystals, especially polymeric nematics. Although the rheological behaviors of LCP's in shear flows have been well documented in experiments, the corresponding theories to interpret 
these phenomena lagged much. Below, we will present three important theories, namely Leslie-Ericksen phenomenological theory, Doi's molecular theory, and more recently Leonov's viscoelastic nematodynamic theory for liquid crystalline polymers.

\subsubsection{Leslie-Ericksen Theory}

The Leslie-Ericksen phenomenological theory, considering the spatial variation of the director, has succeeded in interpreting the dynamic behaviors of low-molecularweight thermotropic nematic liquid crystals [73-76]. Although the Leslie-Ericksen theory does not consider the nonlinear viscoelasticity of LCP's, it is very useful for explaining some of the experimental observation made with LCP's, particularly for describing the effect of polydomain textures on the rheological properties of LCP's.

The Ericksen theory [73] was developed for the viscous stresses, which was later combined with Frank theory for the elastic stresses by Leslie [74]. The Leslie-Ericksen constitutive equation for the viscous stress tersor $\underline{\underline{\sigma}}$ is

$$
\begin{aligned}
& \underline{\underline{\sigma}}=\alpha_{1} \underline{n n n n}: \underline{\underline{D}}+\alpha_{2} \underline{n} \underline{N}+\alpha_{3} \underline{N n}+\alpha_{4} \underline{\underline{D}}+\alpha_{5} \underline{n n} \cdot \underline{\underline{D}}+\alpha_{6} \underline{\underline{D}} \cdot \underline{n n} \\
& \underline{N} \equiv \underline{\dot{n}}-\underline{n} \cdot \underline{\underline{\omega}}
\end{aligned}
$$

Here the strain rate tensor $\underline{\underline{D}}$ and vorticity $\underline{\underline{\omega}}$ are defined via velocity gradient $\underline{\nabla \mathrm{v}}$ as:

$$
\underline{\underline{D}}=\frac{1}{2}\left(\underline{\nabla} \underline{\mathrm{v}}+(\underline{\nabla} \underline{\mathrm{v}})^{T}\right), \quad \underline{\underline{\omega}}=\frac{1}{2}\left(\underline{\nabla} \underline{\mathrm{v}}-(\underline{\nabla} \underline{\mathrm{v}})^{T}\right)
$$

As seen that equation (2-1) contains six viscosities $\alpha_{i}$. Parodi derived the following expression [10]

$$
\alpha_{6}=\alpha_{2}+\alpha_{3}+\alpha_{5}
$$

Therefore only five of these visocisties are independent. 
When the director field is non-uniform, Frank distortional stresses affect the rate of rotation of the director. In this case, a new equation for $\underline{\dot{n}}$ obtained from the balance of angular momentum is:

$$
\underline{h}-\underline{n n} \cdot \underline{h}-\gamma_{2}(\underline{n} \cdot \underline{\underline{D}}-\underline{n n} \underline{n}: \underline{\underline{D}})-\gamma_{1} \underline{N}=0
$$

Here the molecular field, vector $\underline{h}$ depends on the same director gradients that generate the distortional energy $\mathrm{W}_{\mathrm{d}}$. Specifically,

$$
h_{i} \equiv \frac{\partial}{\partial x_{j}}\left(\frac{\partial W_{d}}{\partial n_{i, j}}\right)-\frac{\partial W_{d}}{\partial n_{i}}
$$

The splay, twist, and bend portions of $h$ are given as follows [5]:

$$
\begin{aligned}
& \underline{h}_{S}=K_{1} \underline{\nabla}(\underline{\nabla} \cdot \underline{n}) \\
& \underline{h}_{T}=-K_{2}[A \underline{\nabla} \times \underline{n}+\underline{\nabla} \times(A \underline{n})] \\
& \underline{h}_{B}=K_{3}[B \times(\underline{\nabla} \times \underline{n})+\underline{\nabla} \times(\underline{n} \times \underline{B})]
\end{aligned}
$$

where $A \equiv \underline{n} \cdot(\underline{\nabla} \times \underline{n})$ and $\underline{B} \equiv \underline{n} \times(\underline{\nabla} \times \underline{n})$. In the single-constant approximation $\left(K=K_{1}=K_{2}=K_{3}\right), \underline{h}$ is given by

$$
\underline{h}=K \nabla^{2} \underline{n}
$$

In equation (2-5), $\gamma_{1}$ and $\gamma_{2}$ can be expressed in terms of the Leslie viscosities by

$$
\gamma_{1} \equiv \alpha_{3}-\alpha_{2}, \gamma_{2} \equiv \alpha_{6}-\alpha_{5}
$$

If the molecular field $\underline{h}$ is zero, equation (2-5) can be reduced to

$$
\underline{n} \cdot \underline{\underline{D}}-\underline{n} \underline{n} \underline{n}: \underline{\underline{D}}=\lambda \underline{N} ; \quad \lambda=-\frac{\gamma_{2}}{\gamma_{1}}=-\frac{\alpha_{6}-\alpha_{5}}{\alpha_{3}-\alpha_{2}}=\frac{\alpha_{2}+\alpha_{3}}{\alpha_{2}-\alpha_{3}}
$$

Here $\lambda$ is the "tumbling" parameter. 
In a shearing flow, the nematic viscosities $\alpha_{2}$ and $\alpha_{3}$ determine director torques in the orientations. If $\alpha_{2} / \alpha_{3}$ is positive, the nematics in shear flows are called "flow aligning", whereas in the opposite case "tumbling nematics". The viscosity $\alpha_{4}$ is not related to the director torque, but instead determines the viscosity of the fluid when the director is oriented in the vorticity direction. When $\mathrm{De}<<1$, molecular elasticity is negligible and Frank elasticity is important. The flow properties of polymeric nematics can, in principle, be described by the Leslie-Ericksen equations. The occurrence of "polydomain" in LCP's is explained by existing discontinuities (defects or disclinations) throughout the sample. Therefore, the experimental data such shear stress and normal stress are actually the averages over the director spatial distribution in the regions. As a consequence, most macroscopically observable quantities, including the stresses, are in fact averages over the director spatial distribution in regions. The interplay between director and velocity gradient generates a viscous stress, which on the phenomenological level is characterized by five independent viscosities, known as Leslie coefficients.

\subsubsection{Doi Theory}

The molecular theory of Doi and coworkers $[70,71,78,79]$ based on the concept of rigid rod-like polymers for dilute solutions developed by Kirkwood and Auer [83], describes the rheological behavior of concentrated solutions of monodomain lyotropic LCP's consisting of rigid rod-like macromolecules. Doi and Edwards were inspired by reptation model, whose concept was first introduced by de Gennes [77] and has proved to be very useful in modeling the complex dynamics of macromolecules in melts and concentrated solutions. The reptation model considers the motion of a polymeric chain 
confined in a tube-like region, while Doi and Edwards model analyzes the motion of a rigid rod polymer in the tube.

In Doi theory, a Smoluchowski equation is derived for the probability $\psi(u)$ that a rod-like molecule is oriented parallel to a unit vector $\mathbf{u}$ :

$$
\frac{\partial \psi}{\partial t}+\frac{\partial}{\partial \underline{u}} \cdot[(\underline{u} \cdot \underline{\nabla} \underline{\mathrm{v}}-\underline{u} \underline{u} \underline{u}: \underline{\nabla} \underline{\mathrm{v}}) \psi]-\overline{D_{r}} \frac{\partial}{\partial \underline{u}} \cdot\left[\frac{\partial \psi}{\partial \underline{u}}+\psi \frac{\partial}{\partial \underline{u}}\left(\frac{V_{n e m}}{k_{B} T}\right)\right]=0
$$

Here $\underline{\nabla} \underline{\mathrm{v}}$ is the velocity gradient, $\partial / \partial \underline{u}$ is the gradient operator on the unit sphere, $V_{n e m}$ is the nematic potential (Onsager potential), and $\overline{D_{r}}$ is an averaged molecular rotary diffusivity in the nematic phase:

$$
\frac{1}{\overline{D_{r}}} \equiv D_{r}^{-1}\left(\frac{4}{\pi}\right)^{2}\left[\int \psi(\underline{u}) \psi\left(\underline{u^{\prime}}\right) \sin \left(\underline{u}, \underline{u^{\prime}}\right) d^{2} \underline{u} d^{2} \underline{u^{\prime}}\right]^{2}
$$

Here $D_{r}$ is the rotary diffusivity predicted for a rod in an isotropic solution of rods from the theory of Doi and Edwards [80] for semidilute solutions, namely,

$$
D_{r}=\beta D_{r 0}\left(c L^{3}\right)^{-2}
$$

Here $D_{r o}$ is the rotary diffusivity for the rod in dilute solution, and $\beta$ is a dimensionless coefficient. According to dilute-solution theory for high-aspect ratio ellipsoidal particles $[80,83], D_{r o}$ is given by

$$
D_{r 0}=\frac{3 k_{B} T(\ln (L / d)-\gamma)}{\pi \eta_{s} L^{3}}
$$

where $\eta_{s}$ is the solvent viscosity, $d$ is the rod diameter, and the constant $\gamma$ can be taken as 0.8 . 
Once $\psi$ is computed from equation (2-11), the birefringence tensor, proportional

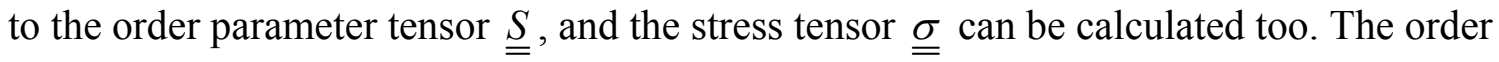
parameter tensor is presented as:

$$
\underline{\underline{S}} \equiv\left\langle\underline{u} \underline{u}-\frac{1}{3} \underline{\delta}\right\rangle
$$

here $\underline{\underline{\delta}}$ is the unit tensor, and $\langle\ldots\rangle$ denotes the average over the distribution function $\psi$.

The scalar order parameter $\mathrm{S}$ is related to the order parameter tensor $\underline{\underline{S}}$ as:

$$
S \equiv \frac{3}{2}(\underline{\underline{S}}: \underline{\underline{S}})^{1 / 2}
$$

It should be noted that $\mathrm{S}$ lies in the range $0-1$; in the extreme cases, $S=0$ for a completely isotropic distribution of orientations and $\mathrm{S}=1$ for rods that are all aligned in the same direction.

The stress tensor is given by [80]

$$
\underline{\underline{\sigma}}=3 v k_{B} T S+v \int \psi\left(\frac{\partial}{\partial u} V_{n e m}\right) \underline{u} d^{2} \underline{u}+v \zeta_{s t r} D:\langle\underline{u} \underline{u} \underline{u} \underline{u}\rangle
$$

The first two terms in equation (2-17) for the stress tensor are elastic terms due to Brownian motion and the nematic potential, respectively. The last, purely viscous term is produced by the drag of solvent as it flows past the rod-like molecules. Here $\zeta_{s t r}$ is a drag coefficient, which for modestly concentrated solutions is predicted to follow the dilutesolution formula [80]:

$$
\zeta_{s t r}=\frac{\pi \eta_{s} L^{3}}{6[\ln (L / d)-\gamma]}=\frac{k_{B} T}{2 D_{r 0}}
$$

The steady shear flow properties can be predicted as [70]: 


$$
\begin{aligned}
& \eta=\frac{v k_{B} T}{6 \bar{D}_{r}}\left\{\frac{(1-S)^{2}(1+2 S)(1+1.5 S)}{(1+0.5 S)^{2}}\right\} \\
& N_{1}=\frac{v k_{B} T}{2 \bar{D}_{r}}\left\{\frac{S(1+2 S)^{1 / 2}(1-S)^{3 / 2}(1+1.5 S)}{(1+0.5 S)^{2}}\right\} \dot{\gamma} \\
& N_{2}=\frac{v k_{B} T}{4 \bar{D}_{r}}\left\{\frac{S^{2}(1+2 S)^{1 / 2}(1-S)^{3 / 2}}{(1+0.5 S)^{2}}\right\} \dot{\gamma}
\end{aligned}
$$

Here $\bar{D}_{r}$ can be expressed as: $\quad \bar{D}_{r}=\frac{\beta D_{r 0}}{\left(c L^{3}\right)^{2}\left(1-S^{2}\right)^{2}}$

With $\beta$ being a dimensionless coefficient.

Equations (2-20) and (2-21) yield $\frac{N_{2}}{N_{1}}=-\frac{S}{2(1+1.5 S)}$

When $S=0$, there is a relationship between $\eta\left(\right.$ or $\mathrm{N}_{1}$ ) and the molecular weight $\mathrm{M}$. It follows from the equations (2-19) and (2-21) with the aid of equations (2-13) and (2-22), yielding $\eta \propto M^{6}$ and $N_{1} \propto M^{6}$.

The Doi theory applies at relatively high flow rates when the elastic stress due to gradients of the director field is negligible. The monodomain assumption, however, precludes the theory from accounting for the generation and proliferation of disclinations at lower flow rates. It was found, however, that the rheological features predicted by Doi theory, have been observed in PBG and HPC solutions only within a range of medium concentrations: $10-25 \%$ for PBG and up to 50\% for HPC. Surprisingly, the Doi rigid-rod statistical theory is incapable to describe the rheological behavior of "rigid rod" polymers [84], such as PBZT [85], and PBO [86]. 
Based on the Doi theory, Marrucci and Maffettone [67] introduced a twodimensional model to predict the transient shear flow behavior. In this system, the Smoluchowski equation is expressed as

$$
\frac{\partial f}{\partial t}=D \frac{\partial}{\partial \Theta}\left(\frac{\partial f}{\partial \Theta}+\frac{f}{k T} \frac{\partial V}{\partial \Theta}\right)-\frac{\partial}{\partial \Theta}(\dot{\Theta} f)
$$

where $D$ is the rotational diffusivity, $f$ is the two-dimensional orientational distribution function, $\dot{\Theta}=-\dot{\gamma} \sin ^{2} \Theta$, and $V$ is the Maier-Saupe nematic potential:

$$
V=-2 U k T\langle\mathbf{u u}\rangle: \mathbf{u u}
$$

Here $k$ is the Boltzmann constant, $\mathrm{T}$ is the absolute temperature, $\mathrm{U}$ is the nondimensional intensity of the potential, and $\mathbf{u}$ is the orientational unit vector.

Since $\Theta=\alpha+\phi$, where $\alpha$ is the angle of director orientation and $\phi$ is the angle which a rod makes with the director, equation (2-24) can be rewritten as

$$
\frac{\partial f}{\partial t}=\frac{\partial^{2} f}{\partial \phi^{2}}+2 U \frac{\partial}{\partial \phi}(f\langle\cos 2 \phi\rangle \sin 2 \phi)+\frac{\partial}{\partial \phi}\left\{f\left[\dot{\alpha}+2 G \sin ^{2}(\alpha+\phi)\right]\right\}
$$

Here time has been non-dimensionalized using the diffusion constant $D$, and $G=\dot{\gamma} / 2 D$ is nondimensional shear rate. In equation (2-26), $\dot{\alpha}=d \alpha / d t$ represents the angular velocity of the director. Equation (2-26) multiplied by $\sin 2 \phi$ and integrated over $\phi$ gives the expression of $\dot{\alpha}$ in terms of the following averages:

$$
\frac{d \alpha}{d t}=G\left[\cos 2 \alpha \frac{\left\langle\cos ^{2} 2 \phi\right\rangle}{\langle\cos 2 \phi\rangle}-\sin 2 \alpha \frac{\langle\sin 2 \phi \cos 2 \phi\rangle}{\langle\cos 2 \phi\rangle}-1\right]-2 U\langle\sin 2 \phi \cos 2 \phi\rangle
$$

By solving equations (2-26) and (2-27), the elastic $\sigma^{E}$ and viscous $\sigma^{v}$ stresses are calculated through the averages as [68]: 


$$
\begin{aligned}
& \frac{\sigma_{12}^{E}}{c k_{B} T}=\langle\cos 2 \phi\rangle\left[\sin 2 \alpha\left(1-U\left\langle\sin ^{2} 2 \phi\right\rangle\right)+\frac{1}{2} U \cos 2 \alpha\langle\sin 4 \phi\rangle\right] \\
& \frac{\sigma_{11}^{E}-\sigma_{22}^{E}}{c k_{B} T}=2\langle\cos 2 \phi\rangle\left[\cos 2 \alpha\left(1-U\left\langle\sin ^{2} 2 \phi\right\rangle\right)-\frac{1}{2} U \sin 2 \alpha\langle\sin 4 \phi\rangle\right] \\
& \frac{\sigma_{12}^{v}}{c k_{B} T}=\beta G[1-\cos 4 \alpha\langle\cos 4 \phi\rangle+\sin 4 \alpha\langle\sin 4 \phi\rangle] \\
& \frac{\sigma_{11}^{v}-\sigma_{22}^{v}}{c k_{B} T}=2 \beta G[\sin 4 \alpha\langle\cos 4 \phi\rangle+\cos 4 \alpha\langle\sin 4 \phi\rangle]
\end{aligned}
$$

Here $\beta=D / D_{0}$ is the diffusivity ratio and $D_{0}$ is the rod diffusivity in dilute solutions.

For numerical analysis, equations (2-26) and (2-27) were first solved for various monodomains using different values of initial condition $\alpha_{0}$, and then the responses of the individual mondomains were averaged to give the response of a polydomain, using equations (2-28)-(2-31). Because the Marrucci-Maffettone analysis is based on the Maier-Saupe potential, it may be applicable to low-molecular-weight liquid crystals.

On the other hand, Larson [69] numerically solved the Doi equation [70] for the three-dimensional time-dependent orientation-distribution function for rodlike polymers in the nematic liquid crystalline state in the presence of shearing flow,

$$
\frac{\partial b_{l m}}{\partial t}=-\left\{\operatorname{lm} \mid \frac{\partial}{\partial u} \cdot([\mathbf{u} \cdot \nabla \mathbf{v}-\mathbf{u u u}: \nabla \mathbf{v}] \psi)\right\}+\bar{D}_{r} l(l+1) b_{l m}+\frac{\bar{D}_{r}}{k T}\left(\operatorname{lm} \mid \frac{\partial}{\partial \mathbf{u}}\left[\psi \frac{\partial}{\partial \mathbf{u}} V_{e v}\right]\right)
$$

where $\mathbf{u}$ is the unit vector, $\nabla \mathbf{v}$ is the velocity gradient,

$$
\bar{D}_{r}=D_{r}\left(1-8 \pi \sum_{\substack{l=2 \\ \text { even }}}^{\infty} \sum_{m=0}^{l}\left(\frac{l+1}{l+2}\right)\left[\frac{(l-3) ! !}{l ! !}\right]^{2}\left|b_{l m}\right|^{2}\right)^{-2} \text { is an orientation-independent rotary }
$$


diffusivity [71], $\frac{\partial}{\partial \mathbf{u}}$ is the gradient operator on the unit sphere, and

$V_{e v}=V_{0}(\mathbf{u})=U k t \int \psi\left(\mathbf{u}^{\prime}\right) \sin \left(\mathbf{u}^{\prime}, \mathbf{u}\right) d^{2} u^{\prime}$ is an effective excluded-volume Onsager potential.

The following notations have been introduced: $\left.(\operatorname{lm} \mid \ldots) \equiv \int \mid \operatorname{lm}\right) \ldots d^{2} u$ is an inner product, $\psi(\mathbf{u})$ is the probability that a thin perfectly rigid rodlike molecule is orientated in parallel to the unit vector $\mathbf{u}$. The Onsager potential is expressed in terms of the spherical harmonic function. Larson's results confirm two-dimensional calculations with the Maier-Saupe potential by Marrucci and Maffettone.

\subsubsection{Leonov's Viscoelastic Nematodynamics}

Unlike the nematic phenomena of low-molecular-weight liquid crystals, the viscoelasticity of liquid crystalline polymers can not be ignored. Instead, it plays a vital role in the rheological properties of LCP's. The viscoelastic characteristics of these systems, being anisotropic, are defined by essentially larger number of constitutive scalar parameters, the experimental determination of which remains a challenging problem. Recently, Leonov [12-14] has a significant breakthrough in viscoelastic nematodyanmics. Specifically, he employed new theoretical techniques [13] to derive the simplest form of the continuum theory of weakly nonlinear viscoelastic nematodynamics. This allows one to determine the minimum number of parameters in the theory using the existing experimental data and predict the rheological behavior of LCP's, which is very important in improving the processing and post-processing properties of LCP's.

To date, there are two theories, continuum and molecular, for solving the problem of modeling nematodynamic properties of LCP's, the molecular theories being not 
developed for thermotropic LCP's. As a matter of fact, these two theories are not contradictory but supplemental. In detail, the continuum theories try to establish a general framework with minimum assumptions of molecular structures, involving, however, many material parameters related to both the basic properties of symmetry and interactions which are described by the state variables. On the contrary, the molecular approaches employing many particular assumptions, describe the LCP properties with few molecular parameters.

As discussed in Chapter 2.3.1, the phenomenological Leslie-Ericksen theory was developed for describing the dynamic behavior of nematic low-molecular-weight thermotropic liquid crystals [73-76]. De Gennes was seemingly first attempted to extend the Leslie-Ericksen theory to the nonlinear case [87]. Unfortunately, the incorporation of nonlinearities into Leslie-Ericksen theory would make the situation even more awkward because of the necessity to increase the number of unkown parameters. Larson and Mead [88] tried to extend the Ericksen phenomenology for flow of low molecular weight liquid crystals to viscoelastic case, using some linear viscoelastic memory functionals instead of Ericksen's viscosities. Because the memory functionals were unknown, Larson and Mead exemplified their approach employing the linearized Doi theory [80,89]. Volkov and Kulichikhin [90] developed a continuum, non-thermodynamics approach to weak anisotropic viscoelasticity of Maxwell type with internal rotations, using symmetry arguments. Further, a thermodynamics theory was developed in references [91,92] for describing linear anisotropic viscoelasticity of LCP's with gradients of state variables. However, a huge number of material parameters, which can not be determined from experimental data, were introduced in these papers, when extending the set of state 
variables with the use of their time-space derivatives. Then, Rey $[93,94]$ employed a particular thermodynamic approach to weak viscoelasticity of LCP's, but his description of asymmetric stress makes his results doubtful. Based on the Kelvin-Voigt type of nematic modeling, Terentjev and Warner [95] proposed a thermodynamic theory of solid viscoelasticity for liquid crystalline elastomers. More recently, Leonov and Volkov $[13,14,96,97]$ initiated thermodynamic studies of nonlinear nematic viscoelasticity for various polymer systems with different rigidity, such as LCP's, LCE's and precursors of polymer nanocomposites.

On the other hand, much effort has also been attracted to the development of molecular theories for modeling the lyotropic LCP's [80,89,98-101]. These theories are based on Doi theory with the same state variables as in case of low molecular weight liquid crystals, including the temperature T, director $\underline{n}$, and the director's space gradient $\underline{\nabla n}$. Additionally, it should be mentioned that Volkov and Kulichikhin [102] and Long and Morse [103] utilized the Rouse-like molecular approaches to LCP's by taking into account the partial flexibility of LCP macromolecular chains, but the closed set of nematodynamic equations have not been obtained in these works.

Golubovich and Lubensky [104] were first to predict the general possibility for occurrence of soft modes in anisotropic elastic solids. Later, Warner and co-authors observed the soft/semi-soft deformation modes for their particular molecular theory for LCE's [6]. Yet these authors were unaware whether the soft modes occurred in their theory by chance or because of a more fundamental reason. Consequtnly, Olmsted [105] tried to justify the occurrence of the soft modes by the rotational invariance, which occurred to be unsuccessful. Lubensky [9] and de Gennes [11] pointed out that the 
occurrence of soft modes in nematic solids can be attributed to the fact that large fluctuations typical for nematics in equilibrium, drive these systems almost to the boundary of their thermodynamic stability where the free energy is effectively minimized not only with respect to state variables but also with respect to material parameters.

Therefore, searching for the "marginal stability" conditions could be used as a theoretical tool for finding the soft deformation modes. This was first introduced by Leonov and Volkov $[106,107]$. Using the marginal stability approach, the possible shearing and elongational soft/semi-soft nematic modes were recently discovered for both the weakly elastic and viscous nematics $[106,107]$. In these systems, the rotational invariance of the shearing modes was found to be a trivial consequence of the marginal stability. Noticeable, the weak Warner elastic potential does not predict the soft elongation mode in the linear limit. The identical results of marginal stability analyses obtained for both viscous and weakly elastic nematic theories [106,107], result from the fact that the well-known LEP continuum theory of nematic LC's has a complete continual analog for weakly elastic LCE's, when the Rayleigh dissipative function in LCP theory is changed for the de Gennes monodomain elastic potential. Since the minimum of free energy functional always exists for all elastic solids, this analogy was additionally justified by demonstrating the principle of minimum of dissipation functional for viscous nematics [108]. It should also be noted that in theories describing possible soft nematic deformation modes, the number of material parameters is highly reduced. The detailed derivation of Leonov's viscoelastic nematodynamics will be given in Chapter IV. 


\section{CHAPTER III}

\section{EXPERIMENTAL DATA AND MATERIALS CHARACTERISTICS OF LIQUID CRYSTALLINE POLYMERS USED IN SIMULATION}

\subsection{Introduction}

A lot of rheological data of liquid crystalline polymers with nematic mesophases have been reported in the literature. Therefore, it is very important to judiciously choose the most reliable and representative data for purpose of our further simulations using Leonov's theory viscoelastic nematodynamics. The chosen rheological data of liquid crystalline polymers should include steady shear flow, transient shear flow, and relaxation data. We chose the rheological data of two commercial LCP's, Titan and Zenite 6000 [63], as well as two academic polymers [59] (a main-chain LCP, PSHQ9 and a side-chain LCP, PI-14-5CN).

3.2 Molecular Characteristics and Rheological Properties of Titan and Zenite 6000 As described by Belatreche [63], two commercial random copolyesters, Titan and Zenite 6000, were obtained from the Eastman and Dupont Chemical companies, respectively. Specifically, Titan is random copolyester probably composed of two random units, ethylene-terephthalate (PET) and hydroxybenzoic acid (HNA). The incorporation of ethylene-terephthalate unit in the main chain reduces the rigidity of the molecule due to the two methylene flexible spacers, and in turn decreases the melting 
temperature of the material. On the other hand, Zenite 6000 is fully aromatic, where kinks were introduced by combining phenol and biphenol molecules by random copolymerization. As measured from differential scanning calorimetry (DSC), the melting temperature $T_{m}$ of Titan was between 325 and $335^{\circ} \mathrm{C}$, while for Zentie $6000 \mathrm{~T}_{\mathrm{m}}$ was between 340 and $360{ }^{\circ} \mathrm{C}$. TGA measurements indicated that the degradation temperatue $\left(T_{d}\right)$ of Titan is $450{ }^{\circ} \mathrm{C}$.

The rheological measurements were conducted using ARES rheometer. To provide a uniform shear flow field, the cone and plate geometry was used with the gap between the cone and lower plate of $50 \mu \mathrm{m}$. Particularly, a "cup-like"fixture on the lower plate of ARES was employed to avoid the compression-molding step causing the thermal degradation of the material. It should be mentioned that this design does not affect the static thermal characteristics of fixtures as well as the measurement of transducers, because it was attached to the lower fixture.

Figure 3.1 presents the plots of viscosity $\eta$, first normal stress difference $\mathrm{N}_{1}$, and shear stress $\sigma_{12}$ versus shear rate in the steady shearing flow of Titan at $340{ }^{\circ} \mathrm{C}$. It is seen that the viscosity of Titan follows the description of Onogi \& Osada [64] with existing the three-region flow curve. Surprisingly, the region I exists till the shear rate increases above $1 \mathrm{~s}^{-1}$. As expected, both $\mathrm{N}_{1}$ and $\sigma_{12}$ inceases with growing shear rate.

Figure 3.2 shows the evolution of shear stress $\sigma_{12}$ and first normal stress difference $\mathrm{N}_{1}$ with time $t$ in start up flow and stress relaxation after cessation of steady flow at $\dot{\gamma}=6$ $\mathrm{s}^{-1}$. There is a large overshoot at ca. $40-50 \mathrm{~s}$ for both $\sigma_{12}$ and $\mathrm{N}_{1}$ curves, and the stresses in the material relax fast after cessation of steady shear flow. 


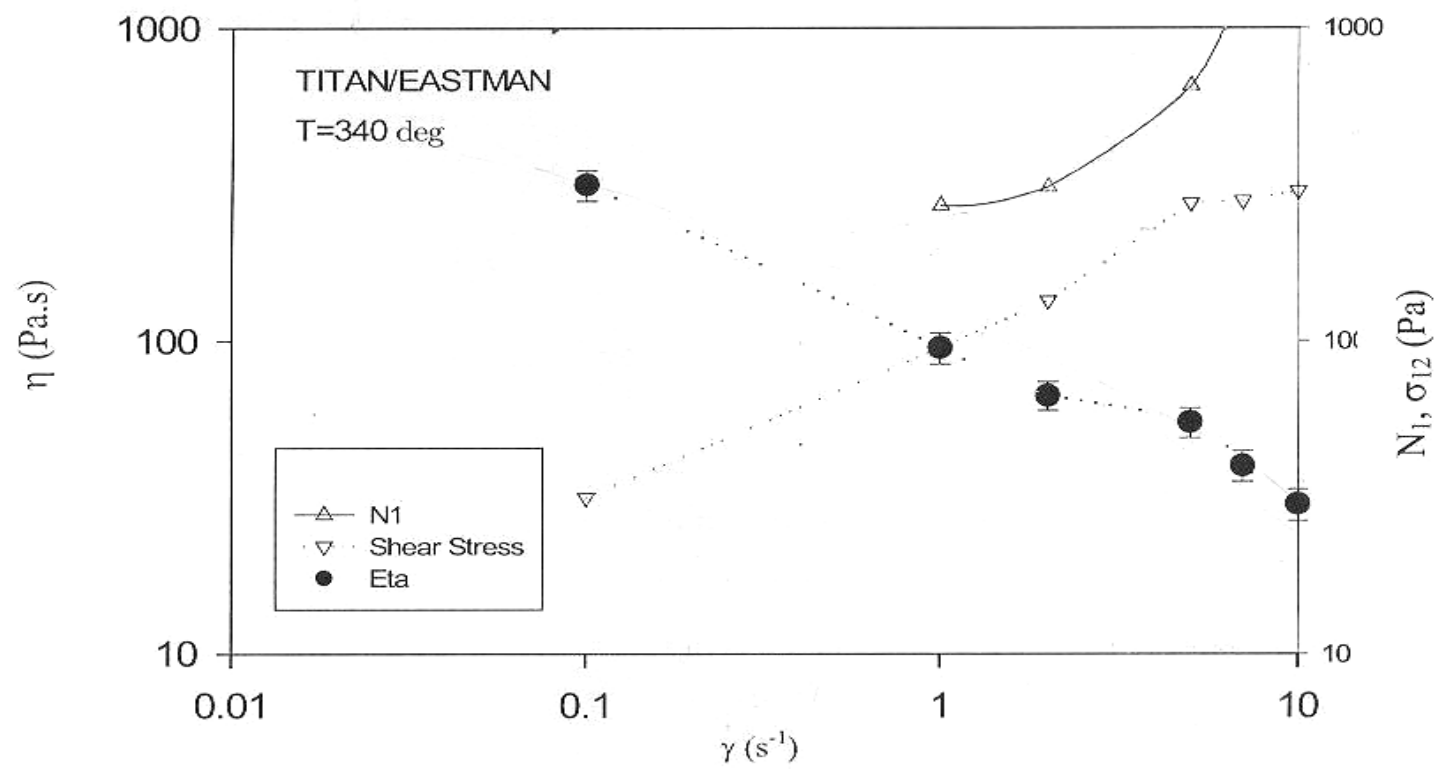

Figure 3.1 Shear stress, normal stress and viscosity in steady flow of Titan at $340{ }^{\circ} \mathrm{C}$ [63].

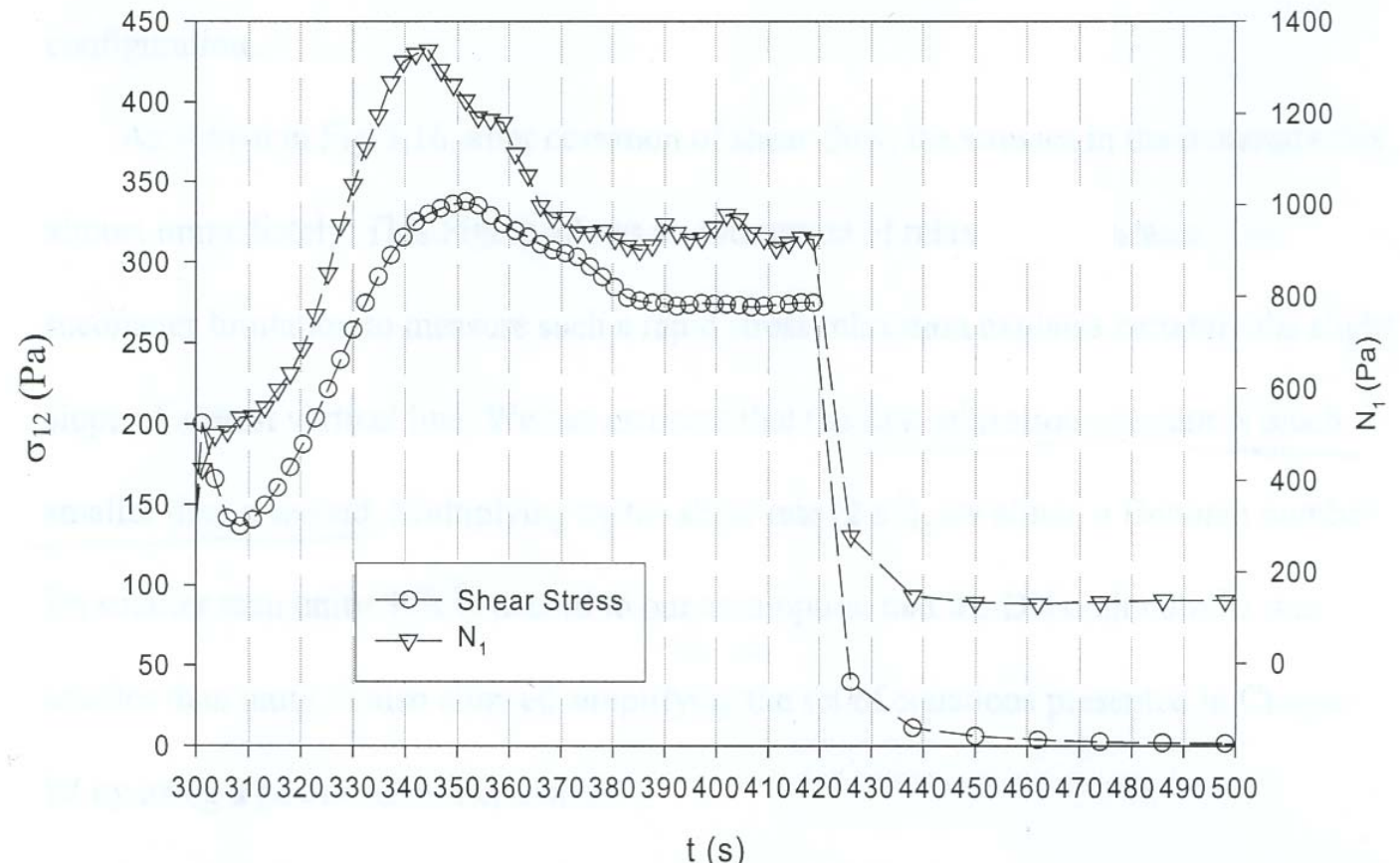

Figure 3.2 Transient shear flow and stress relaxation after cessation of steady shear flow for Titan at $\dot{\gamma}=6 \mathrm{~s}^{-1}[63]$. 
Figure 3.3 presents the plot of viscosity versus shear rate in the steady shear flow for Zenite 6000 . Within the temperature range investigated and shear rate between 0.5 and $3 \mathrm{~s}^{-1}$, only region I and II are observed.

Figure 3.4 shows the evolution of shear stress $\sigma_{12}$ at different shear rates of 1 and 2 $\mathrm{s}^{-1}$ in start up flow for Zenite 6000 at $360{ }^{\circ} \mathrm{C}$. The amplitude and length of the overshoot peaks at different shear rates reveal the nonlinear behavior of the material.

\subsection{Molecular Characteristics and Rheological Properties of PSHQ9 and PI-14-5CN}

The main objective of the model LCP's synthesized in various laboratories is to incorporate flexible spacers or pendent bulky group into the main chain and then reduce the melting point $\left(\mathrm{T}_{\mathrm{m}}\right)$ and the clearing temperature $\left(\mathrm{T}_{\mathrm{cl}}\right)$ of LCP's, which are far below their degradation temperature $\left(T_{d}\right)$. Using these techniques it was possible to safely run the rheological measurements in the temperature intervals below $T_{d}$. One typical example is poly[(phenylsulfonyl)-p-phenylene nonanemethylene bis(4-oxybenzoate)] (PSHQ9) with the chemical structure [59]

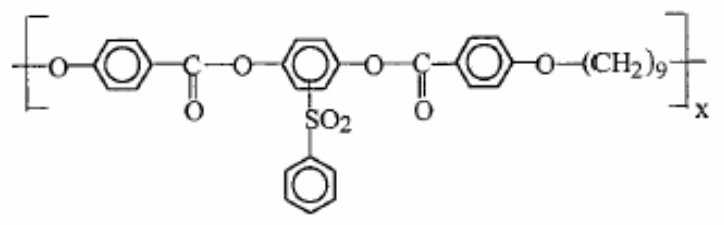

PSHQ9

PSHQ9 is a main-chain liquid-crystalline polymer, which has a glass transition temperature $\left(T_{\mathrm{g}}\right)$ of $84^{\circ} \mathrm{C}$, and a nematic-to-isotropic $(\mathrm{N}-\mathrm{I})$ transition temperature $T_{\mathrm{NI}}$ of $162{ }^{\circ} \mathrm{C}$. It should be mentioned that PSHQ9 has only nematic phase at temperatures between $T_{\mathrm{g}}$ and $T_{\mathrm{NI}}$, and it is a glassy polymer. 


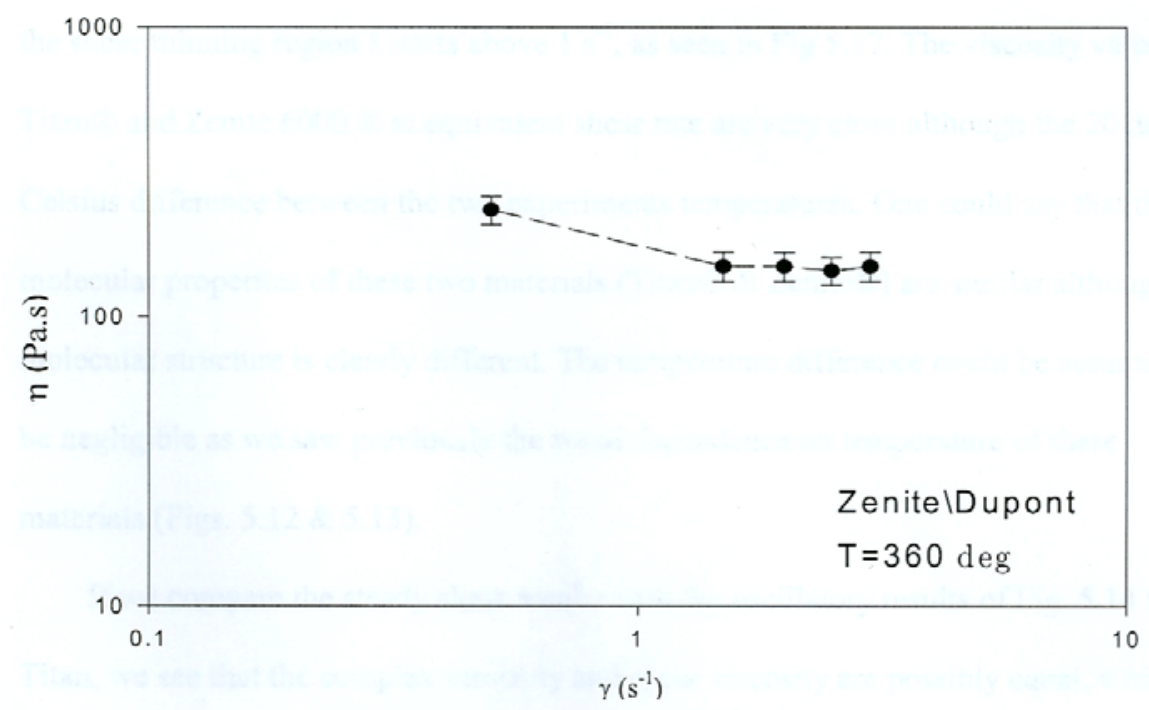

Figure 3.3 Viscosity curve in steady flow of Zenite at $360^{\circ} \mathrm{C}[63]$.

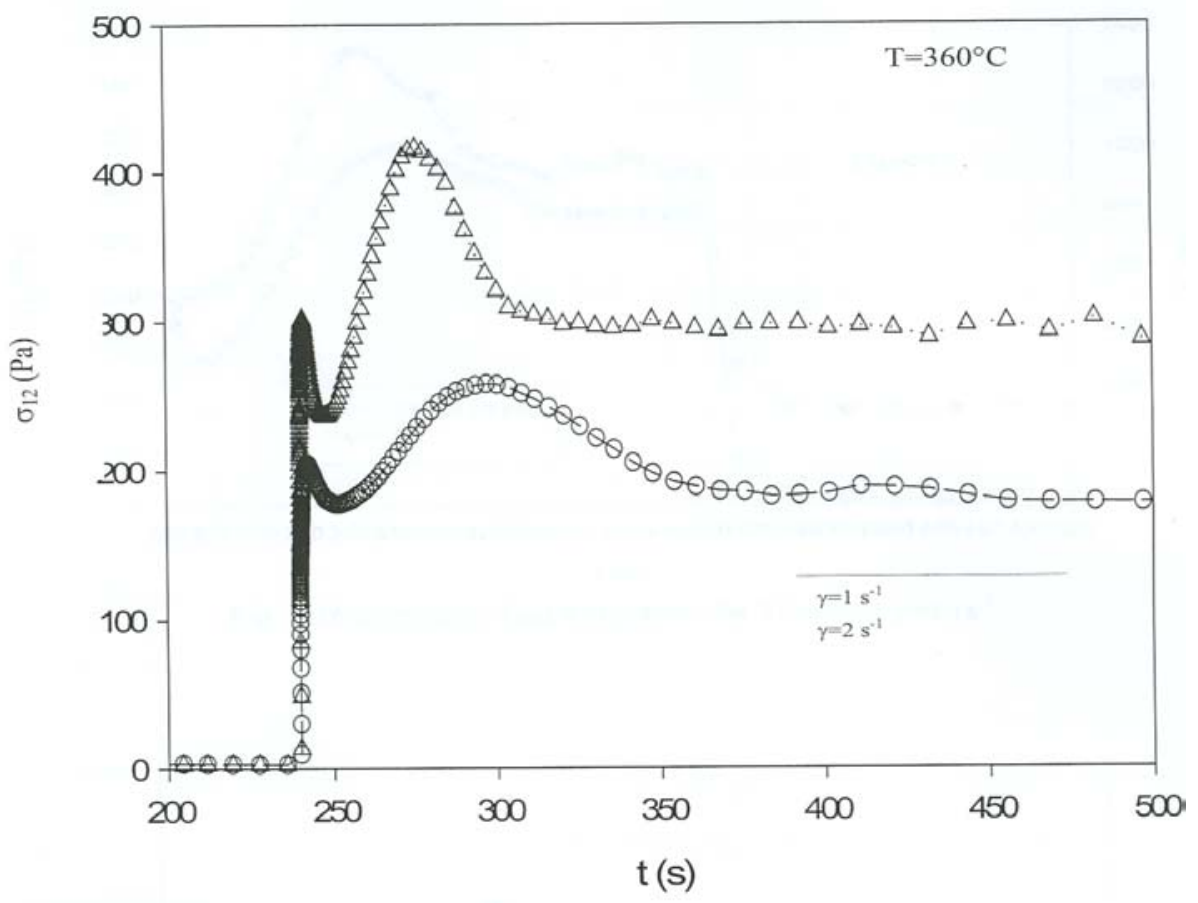

Figure 3.4 Time evolution of shear stress $\left(\sigma_{12}\right)$ at different shear rates $\left(1\right.$ and $\left.2 \mathrm{~s}^{-1}\right)$ in start up flow for Zenite 6000 at $360^{\circ} \mathrm{C}$ [63]. 
Another model polymer, PI-14-5CN is a nematic side-chain liquid-crystalline polymer (SCLCP), with the chemical structure [59]

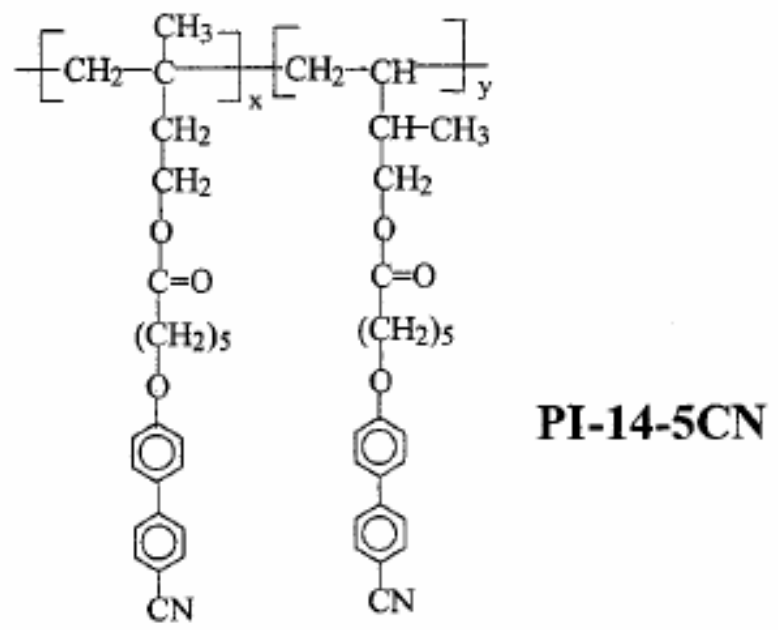

A nearly monodispersed PI-14-5CN was synthesized using anionic polymerization by grafting a liquid-crystalline monomer, 6-[(4-cyano-4'-biphenyl)oxy]hexanoic acid (5CN$\mathrm{COOH}$ ), onto a nearly monodisperse hydroxylated polyisoprene (PI). The PI-14-5CN undergoes glass transition at $45^{\circ} \mathrm{C}$ and N-I transition at $102{ }^{\circ} \mathrm{C}$. The PI- $14-5 \mathrm{CN}$ is a glassy polymer. It is significant that almost all the SCLCP's reported in the literature have been synthesized by condensation polymerization, invariably giving rise to polydisperse SCLCP's. Since an accurate determination of the molecular weight of PI14-5CN from the GPC trace was not possible due to the lack of information on the hydrodynamic volume of PI-14-5CN, the molecular weight of PI-14-5CN was calculated to be $7.19 \times 10^{4} \mathrm{~g} / \mathrm{mol}$ using information on the degree of hydroxylation. The polydispersity of PI-14-5CN determined from GPC is found to be 1.08 .

Samples for rheological measurements were prepared by first dissolving each polymer in THF in the presence of $0.1 \mathrm{wt} \%$ antioxidant (Irganox 1010, Ciba-Geigy Group) and then slowly evaporating the solvent at room temperature for 1 week. The cast 
films ( $1 \mathrm{~mm}$ thick) were further dried in a vacuum oven at room temperature for at least 3 weeks and, prior to measurements, at $60{ }^{\circ} \mathrm{C}$ for $48 \mathrm{~h}$ to remove any residual solvent and moisture. An Advanced Rheometric Expansion System (ARES, Rheometric Scientific) with a cone-and-plate ( $8 \mathrm{~mm}$ diameter plate, $0.1 \mathrm{rad}$ cone angle) fixture and the gap opening at the apex of the cone and plate being set to $50 \mu \mathrm{m}$, was used to investigate steady-state shear flow, transient shear flow, and stress relaxation upon cessation of shear flow. All experiments were conducted under a nitrogen atmosphere to minimize oxidative degradation of the specimens. Temperature control was satisfactory within $\pm 1^{\circ} \mathrm{C}$.

Figure 3.5 shows double logarithmic plots of $\eta$ and $\mathrm{N}_{1}$ versus $\dot{\gamma}$ for PSHQ9 at 130, 140 , and $150{ }^{\circ} \mathrm{C}$ in the nematic region, for $\dot{\gamma}$ ranging from 0.003 to $5 \mathrm{~s}^{-1}$. In the case of $130{ }^{\circ} \mathrm{C}$, the viscosity of PSHQ9 exhibits a shear-thinning behavior at low $\dot{\gamma}$, followed by a Newtonian region and finally strong shear-thinning behavior at higher $\dot{\gamma}$. This is well consistent with the notation of Onogi and Asada [64]. However, as temperature increases, the region I almost disappears.

Figure 3.6 displays the start up shear flow data for PSHQ9 at $140{ }^{\circ} \mathrm{C}$ in the nematic region for $\dot{\gamma}=1.0 \mathrm{~s}^{-1}$. Shown in the Figure are plots of normalized first normal stress difference $N_{1}^{+}(\dot{\gamma}, t) / N_{1}$ and normalized shear stress $\sigma^{+}(\dot{\gamma}, t) / \sigma$ versus strain $\dot{\gamma} t$. As seen from Figure 3.6 that both $N_{1}^{+}(\dot{\gamma}, t) / N_{1}$ and $\sigma^{+}(\dot{\gamma}, t) / \sigma$ go through a large overshoot and then decay to steady values.

Figure 3.7 shows the decay of normalized (a) shear stress $\sigma^{-}(\dot{\gamma}, t) / \sigma_{0}$ and (b) the first normal stress difference with time $t_{R}$ for PSHQ9 at $130{ }^{\circ} \mathrm{C}$. These data were obtained for stress relaxation after cessation of steady shear flows with $\dot{\gamma}=0.1,0.5$, or $1.0 \mathrm{~s}^{-1}$. 


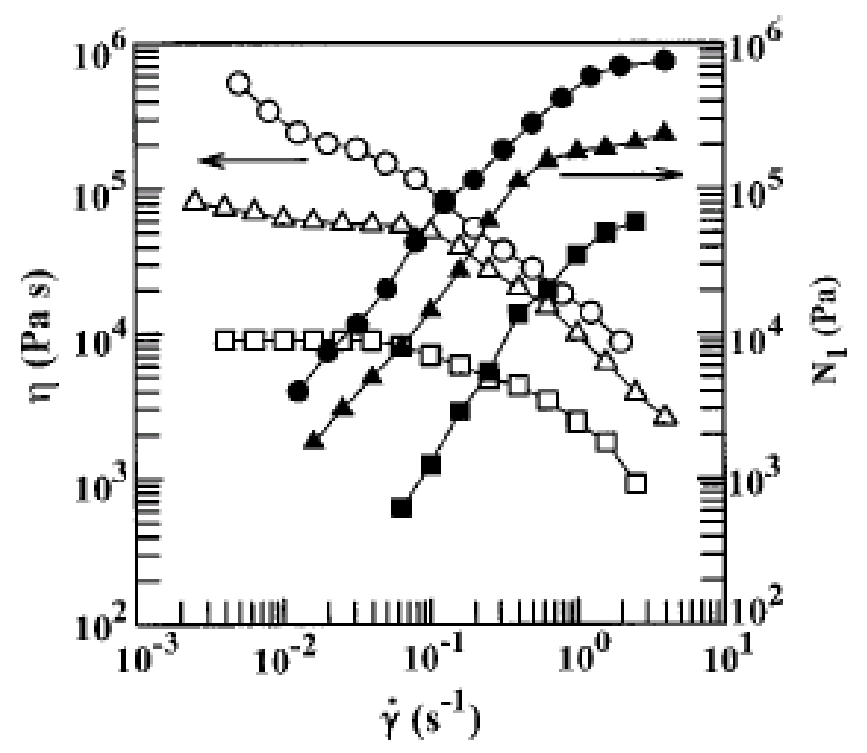

Figure 3.5 Plots of $\log \eta$ vs $\log \dot{\gamma}$ (open symbols) and $\log \mathrm{N}_{1}$ vs $\log \dot{\gamma}$ (filled symbols) for steady flow of PSHQ9 at different temperatures in the nematic region: $(\circ, \bullet) 130^{\circ} \mathrm{C}$; $(\Delta, \mathbf{\Delta}) 140^{\circ} \mathrm{C} ;(\square, \mathbf{\square}) 150^{\circ} \mathrm{C}[59]$.

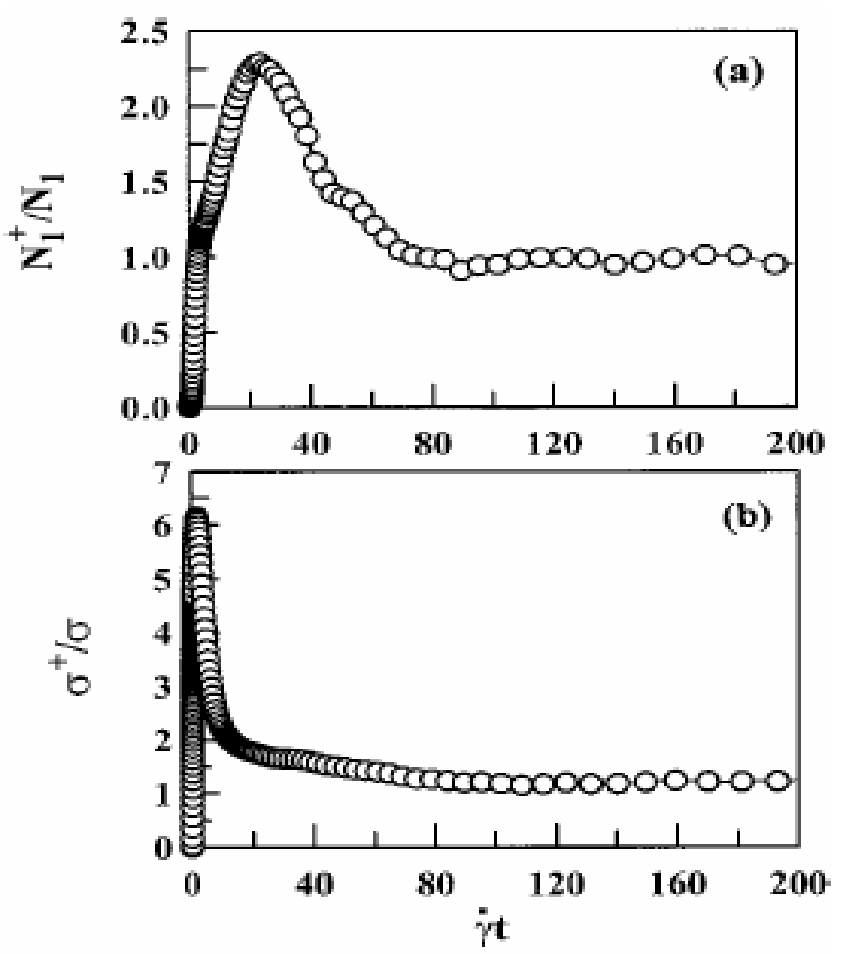

Figure 3.6 The time evolution of (a) $N_{1}^{+}(\dot{\gamma}, t) / N_{1}$ and (b) $\sigma^{+}(\dot{\gamma}, t) / \sigma$ for PSHQ9 at 140 ${ }^{\circ} \mathrm{C}$ in start-up shear flow at $\dot{\gamma}=1.0 \mathrm{~s}^{-1}$ [59]. 
Here $\sigma_{0}$ and $N_{1,0}$ are the steady values of shear stress and first normal stress difference, respectivelty, just prior to flow cessation. As seen from Figure 3.7 the relaxation rates of shear stress and first normal stress difference are getting slower as the shear rate in prior steady shearing increases.

The double logarithmic plots of $\eta$ and $\mathrm{N}_{1}$ versus $\dot{\gamma}$ in steady shearing are presented in Figure 3.8 for PI-14-5CN at $70,80,90^{\circ} \mathrm{C}$ in the nematic region. One can find from

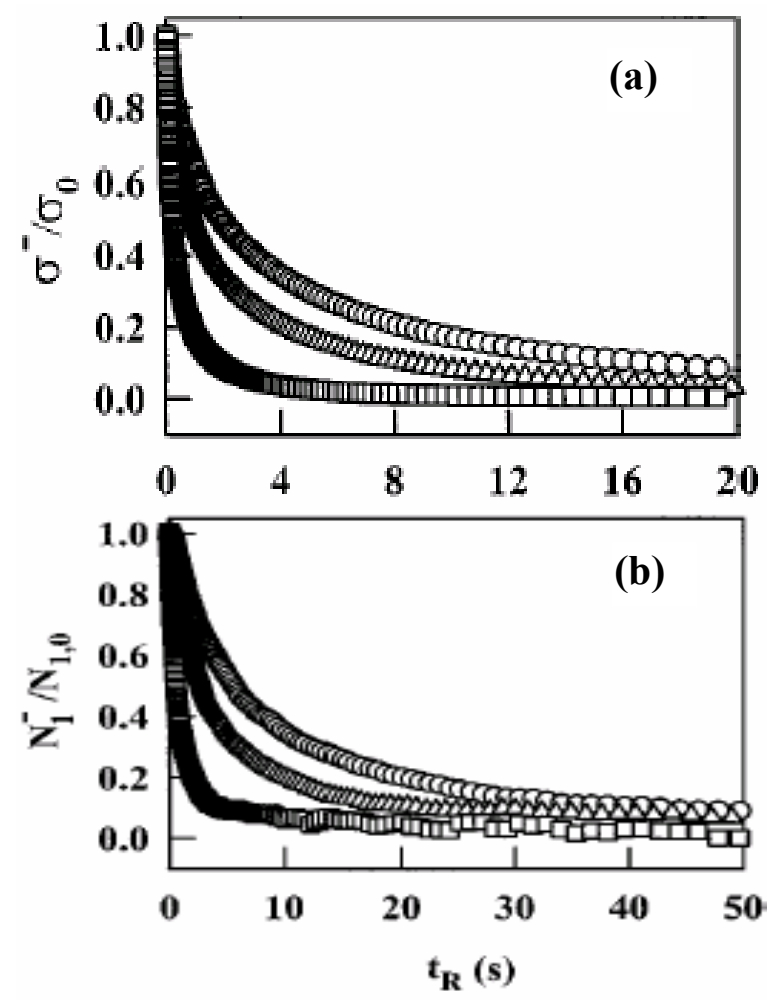

Figure 3.7 Relaxation of normalized (a) shear stress, $\sigma^{-}(\dot{\gamma}, t) / \sigma_{0}$ and (b) first normal stress difference, $N_{1}^{-}(\dot{\gamma}, t) / N_{1,0}$ upon cessation of steady shear flow at different shear rates: (०) $\dot{\gamma}=1.0 \mathrm{~s}^{-1} ;(\Delta) \dot{\gamma}=0.5 \mathrm{~s}^{-1}$; (口) $\dot{\gamma}=0.1 \mathrm{~s}^{-1}$ for PSHQ9 at $130^{\circ} \mathrm{C}$, where $\sigma_{0}$ and $N_{1,0}$ are the steady shear stress and first normal stress difference just prior to flow cessation, respectively [59]. 


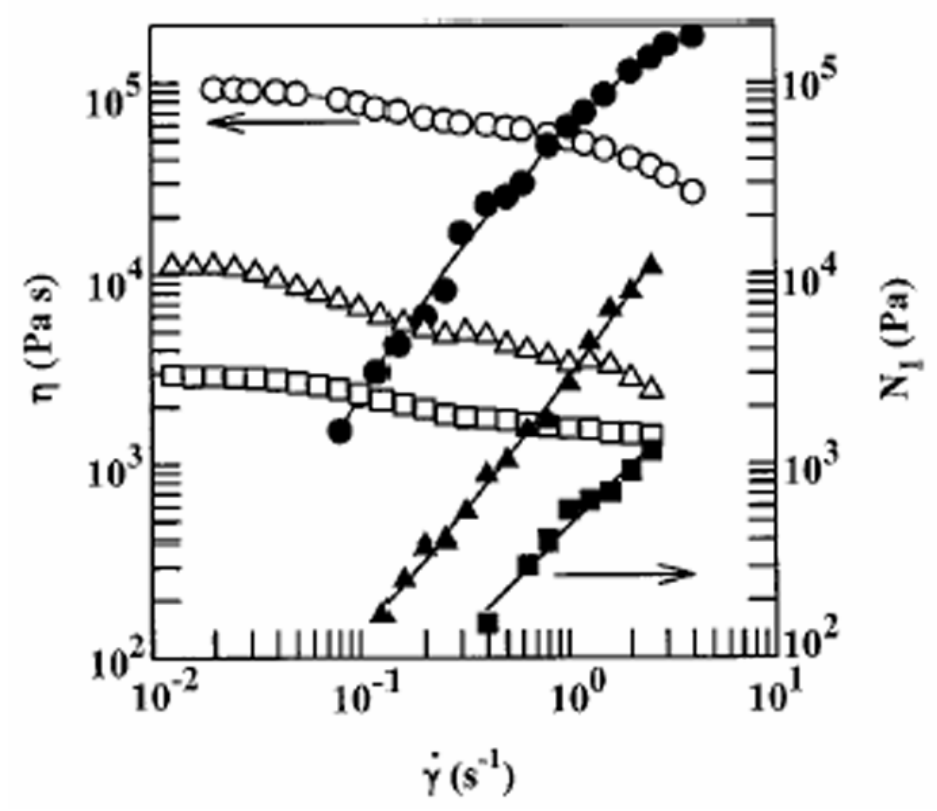

Figure 3.8 Plots of $\log \eta$ vs $\log \dot{\gamma}$ (open symbols) and $\log \mathrm{N}_{1}$ vs $\log \dot{\gamma}$ (filled symbols) for PI-14-5CN in steady flow at different temperatures in the nematic region: $(\circ, \bullet) 70{ }^{\circ} \mathrm{C}$; $(\Delta, \boldsymbol{\Delta}) 80^{\circ} \mathrm{C} ;(\square, \boldsymbol{\bullet}) 90^{\circ} \mathrm{C}[59]$.

Figure 3.8 that the viscosity of PI-14-5CN exhibits a Newtonian behavior at low $\dot{\gamma}$ and then shear-thinning behavior at high $\dot{\gamma}$. It is also clearly seen that $\mathrm{N}_{1}$ is always positive within the entire range of $\dot{\gamma}$ investigated.

Figure 3.9 describes the normalized stress difference $N_{1}^{+}(\dot{\gamma}, t) / N_{1}$ and normalized shear stress $\sigma^{+}(\dot{\gamma}, t) / \sigma$ with strain $(\dot{\gamma} t)$ for PI-14-5CN at $70{ }^{\circ} \mathrm{C}$ in the nematic region during transient shear flow at $\dot{\gamma}=1.0 \mathrm{~s}^{-1}$. Here $N_{1}^{+}(\dot{\gamma}, t)$ is the first normal stress difference growth and $\sigma^{+}(\dot{\gamma}, t)$ is the shear stress growth in start-up shear flow. Obviously, both $N_{1}^{+}(\dot{\gamma}, t) / N_{1}$ and $\sigma^{+}(\dot{\gamma}, t) / \sigma$ go through a large overshoot and then decay to the steady values. 

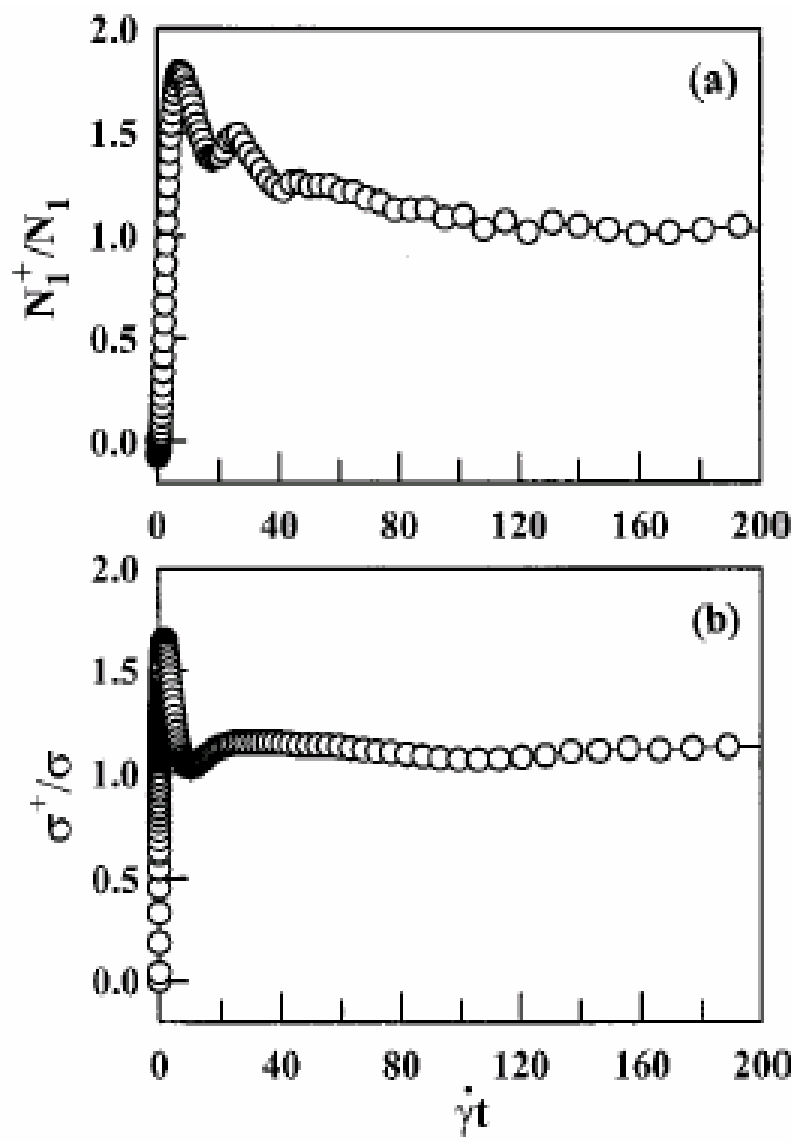

Figure 3.9 Time evolution of (a) $N_{1}^{+}(\dot{\gamma}, t) / N_{1}$ and (b) $\sigma^{+}(\dot{\gamma}, t) / \sigma$ for PI-14-5CN at 70 ${ }^{\circ} \mathrm{C}$ in start-up shear flow at $\dot{\gamma}=1.0 \mathrm{~s}^{-1}$ [59].

Figure 3.10 describes the decay of normalized (a) shear stress $\sigma^{-}(\dot{\gamma}, t) / \sigma_{0}$ and (b) first normal stress difference with time $t_{\mathrm{R}}$ for $\mathrm{PI}-14-5 \mathrm{CN}$ at $70^{\circ} \mathrm{C}$ after cessation of steady shear flow with $\dot{\gamma}=0.1,0.5$, or $1.0 \mathrm{~s}^{-1}$. Here $\sigma_{0}$ and $N_{1,0}$ are the steady values of the shear stress and first normal stress difference just prior to flow cessation, respectively. As compared with PSHQ9 in Figure 3.7, the relaxation rate of both shear stress and first normal stress difference of PI-14-5CN is much faster, and the first normal stress difference upon cessation of shear flow first decreases rapidly and then fluctuates near the zero value. 


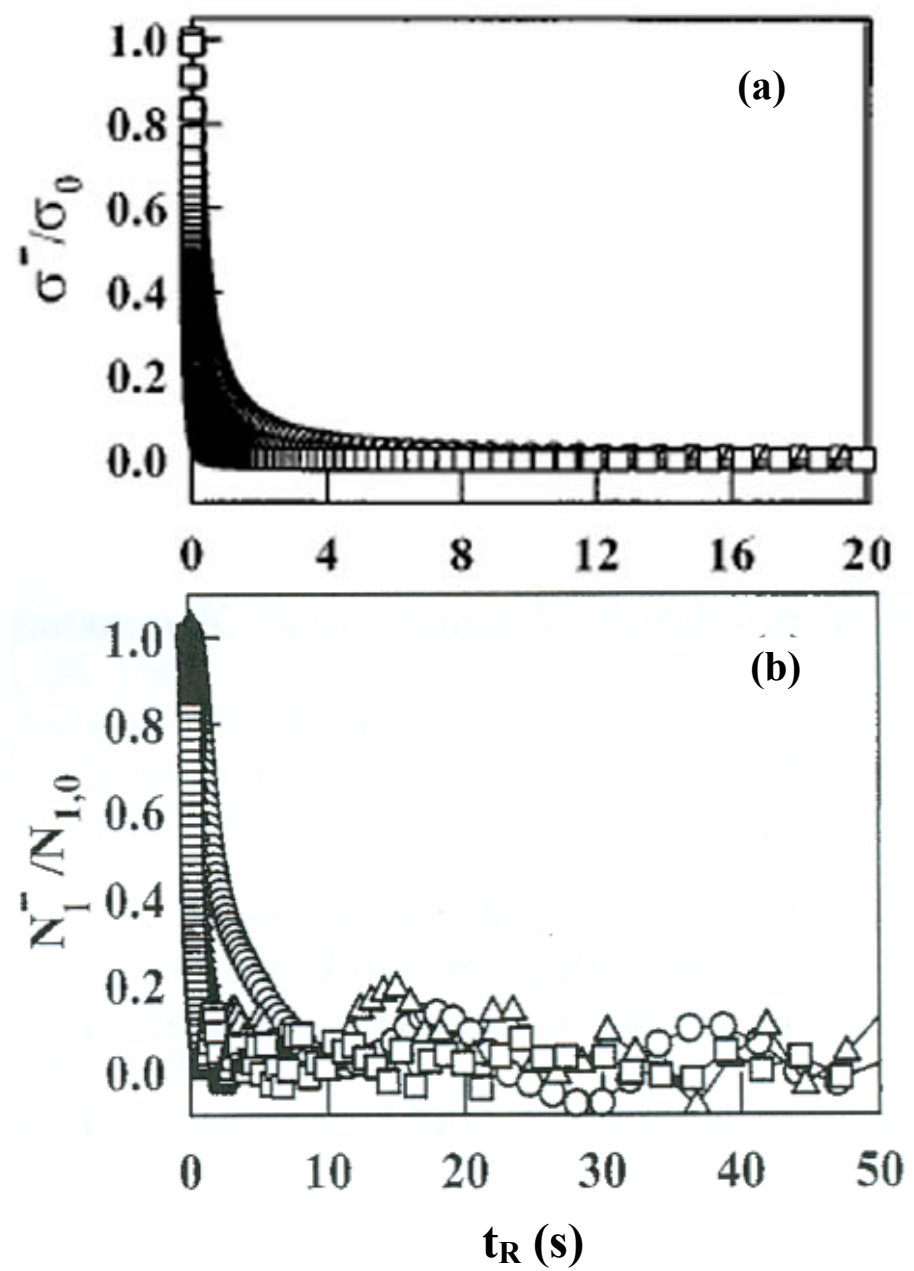

Figure 3.10 Relaxation of normalized (a) shear stress, $\sigma^{-}(\dot{\gamma}, t) / \sigma_{0}$ and (b) first normal stress difference, $N_{1}^{-}(\dot{\gamma}, t) / N_{1,0}$ upon cessation of steady shear flow at different shear rates: (०) $\dot{\gamma}=1.0 \mathrm{~s}^{-1} ;(\Delta) \dot{\gamma}=0.5 \mathrm{~s}^{-1}$; (口) $\dot{\gamma}=0.1 \mathrm{~s}^{-1}$ for PI- $14-5 \mathrm{CN}$ at $70^{\circ} \mathrm{C}$. Here $\sigma_{0}$ and $N_{1,0}$ are the steady shear stress and first normal stress difference just prior to flow cessation, respectively [59]. 


\section{CHAPTER IV}

\section{THEORETICAL MODEL OF SHEAR FLOW OF VISCOELASTIC NEMATOELASTICS}

\subsection{Introduction}

The following simulation will be based on the Leonov's continuum theory of weak viscoelastic nematodynamics of Maxwell type, which can be used to describe the molecular elasticity effects in monodomain flows of liquid crystalline polymers. Along with viscoelastic effects and nematic kinematics, this theory employs a general form of weakly elastic thermodynamic potential and the Leslie-Ericksen-Parodi (LEP) type constitutive equations for viscous nematic liquids, while ignoring inertia effects and the Frank elasticity in liquid crystalline polymers. In general case, even the simplest Maxwell model has many basic parameters. Nevertheless, recently discovered algebraic properties of nematic operations reveal a general structure of the theory and present it in a simple form. It is shown that the evolution equation for director is also viscoelastic. An example of magnetization exemplifies the action of non-symmetric stresses. When the magnetic field is absent, the theory is reduced to the symmetric, fluid mechanical case with relaxation properties for both the stress and director.

\subsection{Thermodynamics and Constitutive Relations}

The theory describes the quasi-equilibrium effects in weak visoelasticity for nematic liquid crystalline polymer in the monodomain case, using de Gennes elastic 
potential (Helmholtz free energy density) [11]:

$$
f=\frac{1}{2} G_{0}|\underline{\underline{\varepsilon}}|^{2}+G_{1} \underline{\underline{n}}: \underline{\underline{\varepsilon}}^{2}+G_{2}(\underline{\underline{n n}}: \underline{\underline{\varepsilon}})^{2}-2 G_{3} \underline{\underline{n n}}:\left(\underline{\underline{\varepsilon}} \cdot \underline{\underline{\Omega}}_{e}\right)-G_{5} \underline{\underline{n n}}:{\underline{\underline{\Omega^{2}}}}_{e}
$$

Here $G_{k}$ are nematic moduli, $\underline{n}$ is director, $\underline{\underline{\varepsilon}}$ is elastic transient strain, and $\underline{\underline{\Omega}}_{e}$ is elastic rotation. Note that the Cosserat, non-nematic isotropic term $\operatorname{tr} \underline{\underline{\Omega}}_{e}^{2}$ is omitted in equation (4-1) because its values in the isotropic case are typically very small.

The well-known expression for the entropy production $P_{s}$ in nonequilibrium systems is given as

$$
T P_{s}=-\underline{q} \cdot \underline{\nabla} T+\underline{\underline{\sigma}}^{s}: \underline{\underline{e}}+\underline{\underline{\sigma}}^{a}: \underline{\underline{\omega}}-\left.d f\right|_{T} / d t
$$

Here $\underline{\underline{\sigma}}^{s}$ and $\underline{\underline{\sigma}}^{a}$ are the the symmetric and asymmetric parts of stress, presented in turn as the sums of equilibrium and non-equilibrium contributions, denoted by the lower indices "e" and "p"as $\underline{\underline{\sigma}}^{s} \equiv \underline{\underline{\sigma}}_{p}^{s}+\underline{\underline{\sigma}}_{e}^{s}, \underline{\underline{\sigma}}^{a} \equiv \underline{\underline{\sigma}}_{p}^{a}+\underline{\underline{\sigma}}_{e}^{a}, \underline{\underline{\sigma}}_{e}^{s}=\partial f / \partial \underline{\underline{\varepsilon}}, \underline{\underline{\sigma}}_{e}^{a}=\partial f / \partial \underline{\underline{\Omega}}_{e}$.

Then the entropy production takes the form:

$$
T P_{s}=-\underline{q} \cdot \underline{\nabla} T+\underline{\underline{\sigma}}_{p}^{s}: \underline{\underline{e}}+\underline{\underline{\sigma}}_{p}^{a}: \underline{\underline{\omega}}+\underline{\underline{\sigma}}_{e}^{s}: \underline{\underline{e}}_{p}+\underline{\underline{\sigma}}_{e}^{a}: \underline{\underline{\omega}}_{p}
$$

Here $\underline{q}$ is the thermal flux, $\mathrm{T}$ is the temperature, and $\underline{\underline{\omega}}_{p}$ is the irreversible relative vorticity. In equation (4-3), $\underline{\underline{\sigma}}^{s}$ and $\underline{\underline{\sigma}}^{a}$ are the symmetric and asymmetric parts of the extra stress tensor, respectively, as given as follows:

$$
\begin{aligned}
& \underline{\sigma^{s}}=\partial f / \partial \underline{\underline{\varepsilon}}=G_{0} \underline{\underline{\varepsilon}}+G_{1}[\underline{n n} \cdot \underline{\underline{\varepsilon}}+\underline{\underline{\varepsilon}} \cdot \underline{n n}-2 \underline{n n}(\underline{\underline{\varepsilon}}: \underline{n n})]+2\left(G_{1}+G_{2}\right)(\underline{n n}-\underline{\underline{\delta}} / 3)(\underline{\underline{\varepsilon}}: \underline{n n}) \\
& +G_{3}\left(\underline{\underline{n n}} \cdot \underline{\underline{\Omega}} \underline{\underline{\Omega}}_{e} \underline{\underline{\Omega}}_{e} \cdot \underline{n n}\right) \\
& \quad \underline{\underline{\sigma}}^{a}=\partial f / \partial \underline{\underline{\Omega}}_{e}=-G_{4}(\underline{n n}: \underline{\underline{\varepsilon}}-\underline{\underline{\varepsilon}} \cdot \underline{n n})+G_{5}\left(\underline{n n} \cdot \underline{\underline{\Omega}}_{e}+\underline{\underline{\Omega}}_{e} \cdot \underline{n n}\right)
\end{aligned}
$$


In the case of the simplest Maxwell type constitutive equation for viscoelastic

nematic liquids, $\underline{\underline{\sigma}}_{p}^{s}=0, \underline{\underline{\sigma}}_{p}^{a}=0$. Then the relation (4-3) for entropy production takes

the form:

$$
T P_{s}=-\underline{q} \cdot \underline{\nabla} T+\underline{\underline{\sigma}}^{s}: \underline{e}_{p}+\underline{\underline{\sigma}}^{a}: \underline{\underline{\omega}}_{p}
$$

Subsequently, the constitutive relations between the irreversible kinematical variables and stress are of the LEP type:

$$
\begin{aligned}
& \underline{\underline{\sigma}}^{s}=\eta_{0} \underline{\underline{e}}_{p}+\eta_{1}\left[\underline{n n} \cdot \underline{\underline{e}}_{p}+\underline{\underline{e}}_{p} \cdot \underline{n n}-2 \underline{n n}\left(\underline{n n}: \underline{\underline{e}}_{p}\right)\right]+2\left(\eta_{1}+\eta_{2}\right)(\underline{n n}-\underline{\underline{\delta}} / 3)\left(\underline{n n}: \underline{e}_{p}\right) \\
& +\eta_{3}\left(\underline{\underline{n}} \cdot \underline{\underline{\omega}}_{p}-\underline{\underline{\omega}}_{p} \cdot \underline{\underline{n}}\right) \\
& \quad \underline{\underline{\sigma}}^{a}=-\eta_{4}\left(\underline{n n} \cdot \underline{\underline{e}}_{p}-\underline{\underline{e}}_{p} \cdot \underline{n n}\right)+\eta_{5}\left(\underline{n n} \cdot \underline{\underline{\omega}}_{p}+\underline{\underline{\omega}}_{p} \cdot \underline{n n}\right)
\end{aligned}
$$

The Onsager relation, $\eta_{3}+\eta_{4}=0$, between the couple terms in equations (4-7) and (4-8) is also used here. The relations between the Leslie-Ericksen parameters $\alpha_{k}$ and the "viscosities" $\eta_{k}$ are given as follows:

$\alpha_{1}=2 \eta_{2}, \alpha_{2}=-\eta_{3}-\eta_{4}, \alpha_{3}=-\eta_{3}+\eta_{4}, \alpha_{4}=\eta_{0}, \alpha_{5}=\eta_{1}+\eta_{3}, \alpha_{6}=\eta_{1}-\eta_{3}$

Here additional Parodi equality $\alpha_{2}+\alpha_{3}=\alpha_{6}-\alpha_{5}=-2 \eta_{3}$ has also been satisfied.

\subsection{Closed Set of Maxwell Viscoelastic Nematodynamics}

The kinematical relation for characterizing the combined effect of viscoelastic deformation and relative rotations is given as [14]:

$$
\stackrel{0}{\underline{\Gamma}}+e_{\underline{\underline{\gamma}}}=\underline{\underline{\gamma}}
$$

Since in the Maxwell liquid the stress is the same in viscous and elastic modes, it can be derived from equations (4-4), (4-7) as well as (4-5), (4-8) as: 


$$
\underline{\underline{\sigma}}=\mathbf{G}(\underline{n}) \bullet \underline{\Gamma}_{e}=\boldsymbol{\eta}(\underline{n}) \bullet \underline{\gamma}_{p}
$$

Here $\mathbf{G}(\underline{n})$ and $\boldsymbol{\eta}(\underline{n})$ are operators of moduli and viscosity, respectively, represented by their $4^{\text {th }}$ rank tensors [14].

Relation (4-11) can be rewritten as:

$$
\underline{\gamma}_{p}=\mathbf{s}(\underline{n}) \bullet \underline{\Gamma}_{e} \text { and } \underline{\underline{\Gamma}}_{e}=\boldsymbol{\theta}(\underline{n}) \bullet \underline{\gamma}_{p}
$$

Here $\mathbf{s}(\underline{n})=\boldsymbol{\eta}^{-1}(\underline{n}) \bullet \mathbf{G}(\underline{n})$ and $\boldsymbol{\theta}(\underline{n})=\mathbf{G}^{-1}(\underline{n}) \bullet \boldsymbol{\eta}(\underline{n})$ are the fourth rank tensors of relaxation frequency and relaxation time, respectively. Substituting equation (4-12) into (4-10) yields the operator form of evolution equation: $\stackrel{0}{\underline{\Gamma}}_{e}+\mathbf{s}(\underline{n}) \bullet \underline{\Gamma_{e}}=\underline{\gamma}$, which is presented as the coupled equations:

$\stackrel{0}{\underline{\varepsilon}}+s_{0} \underline{\underline{\varepsilon}}+s_{1}[\underline{n n} \cdot \underline{\underline{\varepsilon}}+\underline{\underline{\varepsilon}} \cdot \underline{n n}-2 \underline{n n}(\underline{\underline{\varepsilon}}: \underline{n n})]+s_{2}(\underline{n n}-\underline{\underline{\delta}} / 3)(\underline{\underline{\varepsilon}}: \underline{n n})+s_{3}(\underline{n n} \cdot \underline{\underline{\Omega}} e-\underline{\underline{\Omega}} \cdot \underline{n n})=\underline{e}$

where $\underline{\underline{e}}=1 / 2\left[\underline{\underline{\nabla v}}+(\underline{\underline{\nabla v}})^{T}\right]$, and $\underline{\underline{\nabla v}}$ is the velocity gradient tensor.

$$
\underline{\underline{\Omega}} e+s_{4}(\underline{n n} \cdot \underline{\underline{\varepsilon}}-\underline{\underline{\varepsilon}} \cdot \underline{n n})+s_{5}(\underline{n n} \cdot \underline{\underline{\Omega}} e+\underline{\underline{\Omega}} \cdot \cdot \underline{n n})=\underline{\underline{\omega}}
$$

where $\underline{\underline{\omega}}=1 / 2\left[\underline{\underline{\nabla v}}-(\underline{\underline{\nabla v}})^{T}\right]$

Here the parmeters $s_{k}$ are the basis parameters of relaxation frequency tensor $\mathbf{s}(\underline{n})$, which are presented via the basic parameters $G_{k}$ and $\eta_{k}$ in the model as:

$$
\begin{aligned}
& s_{0}=\frac{G_{0}}{\eta_{0}}, s_{1}=\frac{\eta_{5}\left(G_{1} \eta_{0}-G_{0} \eta_{1}\right)+\eta_{3}\left(G_{0} \eta_{3}-G_{3} \eta_{0}\right)}{\eta_{0}\left[\eta_{5}\left(\eta_{0}+\eta_{1}\right)-\eta_{3}^{2}\right]}, s_{2}=\frac{3}{2} \cdot \frac{\left(G_{1}+G_{2}\right) \eta_{0}-G_{0}\left(\eta_{1}+\eta_{2}\right)}{\eta_{0}\left(3 / 4 \eta_{0}+\eta_{1}+\eta_{2}\right)} \\
& s_{3}=\frac{G_{3} \eta_{5}-G_{5} \eta_{3}}{\eta_{5}\left(\eta_{0}+\eta_{1}\right)-\eta_{3}^{2}}, s_{4}=\frac{G_{3}\left(\eta_{0}+\eta_{1}\right)-\eta_{3}\left(G_{0}+G_{1}\right)}{\eta_{5}\left(\eta_{0}+\eta_{1}\right)-\eta_{3}^{2}}, s_{5}=\frac{G_{5}\left(\eta_{0}+\eta_{1}\right)-G_{3} \eta_{5}}{\eta_{5}\left(\eta_{0}+\eta_{1}\right)-\eta_{3}^{2}}
\end{aligned}
$$


The basic parameters $\theta_{k}$ of relaxation tensor $\boldsymbol{\theta}(\underline{n})$ could be obtained from equation (4-15) using double transformation: $\eta_{k} \rightarrow G_{k}, G_{k} \rightarrow \eta_{k}$.

It is convenient to introduce the normalized molecular field $\underline{h}_{+}=\underline{h} / G_{5}$ with its projection $\underline{h}_{+}^{\perp}$ on the normal to director, and the traceless normalized symmetric $\underline{h}_{=}^{s}$ and asymmetric $\underline{\underline{h}}_{+}^{a}$ tensors, which are defined using $\underline{h}_{+}$as:

$\underline{h}_{+}^{\perp}=\underline{h}_{+}-\underline{n}\left(\underline{h}_{+} \cdot \underline{n}\right), \underline{h}_{+}^{s}=1 / 2\left[\underline{h}_{+} \underline{n}+\underline{n} \underline{h}_{+}-2 \underline{n n}\left(\underline{h}_{+} \cdot \underline{n}\right)\right], \underline{h}_{+}^{a}=1 / 2\left(\underline{h}_{+} \underline{n}-\underline{n} \underline{h}_{+}\right)$

When inertial effects of internal rotation are negligible, $\underline{\underline{\sigma}}^{a}=\underline{\underline{h}}^{a}$. Thus, substituting equation (4-16) into (4-5) yields:

$$
\underline{\underline{\Omega}}_{e}=\underline{\underline{h}}^{a}+\lambda_{e}(\underline{\underline{\varepsilon}} \cdot \underline{n n}-\underline{n n} \cdot \underline{\underline{\varepsilon}}) \quad\left(\lambda_{e}=G_{3} / G_{5}\right) .
$$

Equation (4-17) also yields the kinematical expression for $\underline{\underline{\omega}}_{p}$, with substitutions:

$\underline{\underline{\Omega}}_{e} \rightarrow \underline{\underline{\omega}}_{p}, \underline{\underline{\varepsilon}} \rightarrow \underline{\underline{e}}_{p}, \lambda_{e} \rightarrow \lambda_{v}=\eta_{3} / \eta_{5}$. Here $\lambda_{e}$ and $\lambda_{v}$ are the elastic and viscous "tumbling" parameters.

Substituting equation (4-17) into (4-13) yields the closed set of Maxwell viscoelastic nematodyanmics in the non-symmetric case when the magnetic field is presented.

$$
\begin{aligned}
& \underline{\underline{\varepsilon}}+s_{0} \underline{\underline{\varepsilon}}+\widetilde{s}_{1}[\underline{n n} \cdot \underline{\underline{\varepsilon}}+\underline{\underline{\varepsilon}} \cdot \underline{n n}-2 \underline{n n}(\underline{\underline{\varepsilon}}: \underline{n n})]+s_{2}(\underline{n n}-\underline{\underline{\delta}} / 3)(\underline{\underline{\varepsilon}}: \underline{n n})=\underline{\underline{e}}+s_{3} \underline{h}^{s} \\
& \left(\tilde{s}_{1}=s_{1}-\lambda_{e} s_{3}\right) \\
& \stackrel{0}{n}-\lambda_{e}[\underline{\underline{\varepsilon}} \cdot \underline{0} \underline{n}-\underline{0}(\underline{\underline{\varepsilon}}: \underline{n n})-\underline{n}(\underline{\underline{\varepsilon}}: \underline{n} \underline{n})]=\mathbf{b}(\underline{n}) \bullet\left(\lambda_{e} \underline{\underline{e}}+\xi_{1} \underline{\underline{\varepsilon}}\right)+{\stackrel{h}{h^{a}}}_{=} \cdot \underline{n}+\xi_{2} \underline{h}_{+}^{\perp} \\
& \underline{n} \approx \mathbf{b}(\underline{n}) \bullet\left(\lambda_{e} \underline{\underline{e}}+\xi_{1} \underline{\underline{\varepsilon}}\right)+{\underline{h^{a}}}_{+}^{a} \cdot \underline{n}+\xi_{2} \underline{h}_{+}^{\perp}
\end{aligned}
$$


$\underline{\underline{\sigma}}=G_{0} \underline{\underline{\varepsilon}}+\hat{G}_{1}[\underline{n n} \cdot \underline{\underline{\varepsilon}}+\underline{\underline{\varepsilon}} \cdot \underline{n n}-2 \underline{n n}(\underline{\underline{\varepsilon}}: \underline{n n})]+2\left(G_{1}+G_{2}\right)(\underline{n n}-\underline{\underline{\delta}} / 3)(\underline{\underline{\varepsilon}}: \underline{n n})-G_{3} \underline{\underline{h^{s}}}+G_{5} \underline{h^{a}}$

Here $\hat{G}_{1}=G_{1}-G_{3}^{2} / G_{5}$ and $(\mathbf{b}(\underline{n}) \bullet \underline{e})_{i}=b_{i j k}(\underline{n}) e_{k j}=\left(\delta_{i j} n_{k}-n_{i} n_{j} n_{k}\right) e_{k j}$,

$\xi_{1}=\lambda_{e}\left(s_{0}+\hat{s}_{1}+s_{5}\right)-s_{4}, \xi_{2}=s_{5}-\lambda_{e} s_{3}$

4.4 Weak Nematic Viscoelasticity in the Absence of Magnetic Field $(\underline{h}=0)$

In the case of the absence on magnetic field $(\underline{h}=0)$, the stress-elastic strain

relation (4-21) and the evolution equation (4-18) for transient elastic strain take the forms:

$$
\begin{aligned}
& \underline{\underline{\sigma}}=\hat{\mathbf{G}}(\underline{n}) \bullet \underline{\underline{\varepsilon}} \\
& 0 \\
& \underline{\underline{\varepsilon}}+\hat{\mathbf{s}}(\underline{n}) \bullet \underline{\underline{\varepsilon}}=\underline{\underline{e}} \\
& \hat{\boldsymbol{\theta}}(\underline{n}) \bullet \underline{\underline{\varepsilon}}+\underline{\underline{\varepsilon}}=\underline{e}
\end{aligned}
$$

Consider now the approximated evolution equation for director (4-20) with $\underline{h}=0$.

In this case the differentiation with frozen value of $\underline{n}$ is within the approximation of weak nematic viscoelasticity where $|\underline{\underline{\varepsilon}}|<<1$. When $\underline{h}=0$, applying to (4-20) the operator defined in the left-hand side of (4-22b), results in the evolution equation for director:

$$
\begin{gathered}
\theta *\left(\left.\begin{array}{l}
00 \\
\underline{n}+\underline{n} \cdot \mid \underline{n}
\end{array}\right|^{2}\right)+\underline{n}=\mathbf{b}(\underline{n}) \cdot\left(\lambda_{e} \theta * \underline{0} \underline{\underline{e}}+\lambda_{v} \underline{\underline{e}}\right) \\
\operatorname{Here} \theta^{*}=\frac{1}{\hat{s}_{1}+\hat{s}_{2}}=\frac{\eta_{0}+\hat{\eta}_{1}}{G_{0}+\hat{G}_{1}}, \lambda_{e}=\frac{G_{3}}{G_{5}}, \lambda_{v}=\frac{\eta_{3}}{\eta_{5}}
\end{gathered}
$$


Here $\lambda_{e}$ and $\lambda_{v}$ are the elastic and viscous "tumbling" parameters. It is seen that the both sides of (4-23) vanish after scalar multiplication of this equation by $\underline{n}$. Relaxation character of this equation is caused by the "bulk" relaxation due to the viscoelastic nematic character of evolution equation in (4-22) for the transient elastic strain. Note that in particular cases when $\lambda_{e}=\lambda_{v}=\lambda$, as well as when either $\theta^{*} \rightarrow 0$, or $\theta^{*} \rightarrow \infty$, the evolution equation (4-23) reduces to the Ericksen equation, $\underline{n}=\lambda \mathbf{b}(\underline{n}) \cdot \underline{\underline{e}}$.

Using the differentiation with frozen value of $\underline{n}$ one can also exclude the hidden

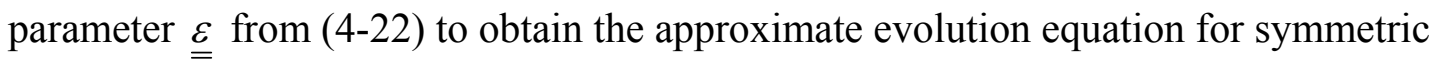
extra stress, written in identical forms:

$$
\begin{aligned}
& \underline{\underline{\sigma}}+\hat{\mathbf{s}}(\underline{n}) \bullet \underline{\underline{\sigma}}=\hat{\mathrm{G}}(\underline{n}) \bullet \underline{\underline{e}} \\
& \hat{\theta}(\underline{n}) \bullet \underline{\underline{\sigma}}+\underline{\underline{\sigma}}=\hat{\mathrm{\eta}}(\underline{n}) \bullet \underline{\underline{e}}
\end{aligned}
$$

Equations (4-22), (4-23), and (4-24) represent the closed set of equations describing symmetric weak viscoelastic nematodynamics of Maxwell type.

\subsection{Soft Deformation Modes in the Weak Maxwell Type Nematodynamics}

Marginal stability approach was used to analyze the soft deformation modes, which could exist only in the absence of external fields $[14,97]$. The soft cases occur when the values of material parameters belong to the marginal stability boundaries $\left(G_{0}>0\right.$,

$\left.G_{0}+\hat{G}_{1}>0,3 / 4 G_{0}+\hat{G}_{1}+\hat{G}_{2}>0 ; \eta_{0}>0, \eta_{0}+\hat{\eta}_{1}>0,3 / 4 \eta_{0}+\hat{\eta}_{1}+\hat{\eta}_{2}>0\right)$. Because of the assumed non-degeneration of constitutive parameters, there are only four independent marginal stability conditions: 


$$
G_{0}+G_{1}=0, \eta_{0}+\eta_{1}=0 ; \frac{3}{4} G_{0}+G_{1}+G_{2}=0, \frac{3}{4} \eta_{0}+\eta_{1}+\eta_{2}=0
$$

The first two equalities in (4-25) are related to the shearing soft modes, and another two to the extensional ones.

We introduce the following definition of the fourth rank symmetric, transversely isotropic traceless, director dependent tensor $\mathbf{A}\left(\underline{n} ; a_{0}, a_{1}, a_{2}\right)$ via the operation of its scalar multiplication by a:

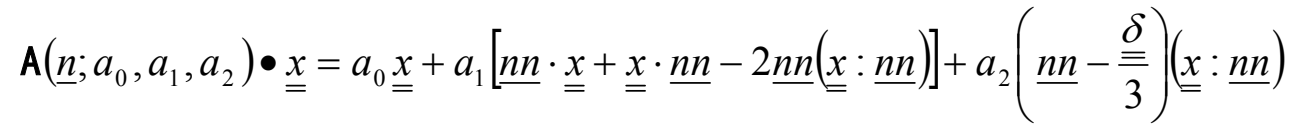

Using (4.26) we can rewrite the basic nematic operators in (4-24) as:

(i) Modulus, $\hat{\mathrm{G}}(\underline{n})=\mathrm{A}\left(\underline{n} ; G_{0}, \hat{G}_{1}, 2 \hat{G}_{1}+2 \hat{G}_{2}\right)$;

(ii) Viscosity, $\hat{\eta}(\underline{n})=\mathbf{A}\left(\underline{n} ; \eta_{0}, \hat{\eta}_{1}, 2 \hat{\eta}_{1}+2 \hat{\eta}_{2}\right)$;

(iii) Relaxation frequency, $\hat{\mathbf{s}}(\underline{n})=\mathbf{A}\left(\underline{n} ; s_{0}, \hat{s}_{1}, \hat{s}_{2}\right)$;

(iv) Relaxation time, $\hat{\theta}(\underline{n})=\mathbf{A}\left(\underline{n} ; \theta_{0}, \hat{\theta}_{1}, \hat{\theta}_{2}\right)$;

Relations (4-27a-d) are helpful to represent constitutive equations (4-24) in explicit form. E.g. equation (4-24a) is rewritten as:

$$
\begin{aligned}
& \stackrel{0}{\underline{\sigma}}+s_{0} \underline{\underline{\sigma}}+s_{1}[\underline{n n} \bullet \underline{\underline{\sigma}}+\underline{\underline{\sigma}} \bullet \underline{n n}-2 \underline{n n}(\underline{\underline{\sigma}}: \underline{n n})]+s_{2}(\underline{n n}-\underline{\underline{\delta}} / 3)(\underline{\underline{\sigma}}: \underline{n n}) \\
& \left.=G_{0} \underline{\underline{e}}+G_{1}[\underline{\underline{n n}} \bullet \underline{\underline{e}}+\underline{\underline{e}} \bullet \underline{\underline{n n}}-2 \underline{n n} \underline{\underline{e}} \underline{\underline{n n}})\right]+2\left(G_{1}+G_{2}\right)(\underline{\underline{n n}}-\underline{\underline{\delta}} / 3)(\underline{\underline{e}}: \underline{n n}) \\
& \text { Here } G_{1}=\hat{a}_{1} G_{0}, 2\left(G_{1}+G_{2}\right)=\left(\hat{\beta}-\frac{3}{2}\right) G_{0}, \hat{\beta}=2\left(\frac{G_{2}}{G_{0}}-\frac{1}{4}\right) \\
& \qquad s_{1}=s_{0}\left(r_{1}-1\right), s_{2}=\frac{3}{2} s_{0}\left(r_{2}-1\right), \theta^{*}=\frac{\eta_{0}+\eta_{1}}{G_{0}+G_{1}}=\frac{\theta_{0}}{r_{1}}
\end{aligned}
$$




$$
\theta_{\beta}=\theta_{0} \frac{\beta}{\hat{\beta}}=\frac{\theta_{0}}{r_{2}}, s_{0} r_{2}=s_{0} \frac{\hat{\beta}}{\beta}=\frac{1}{\theta_{\beta}}, r_{2}=\frac{\hat{\beta}}{\beta}, \mathrm{r}_{1}>0, \mathrm{r}_{2}>0
$$

Then equation (4-28) beomes:

$$
\begin{aligned}
& \underline{\underline{\sigma}}+s_{0}\left\{\underline{\underline{\sigma}}+\left(r_{1}-1\right)[\underline{n n} \cdot \underline{\underline{\sigma}}+\underline{\underline{\sigma}} \cdot \underline{n n}-2 \underline{n n}(\underline{\underline{\sigma}}: \underline{n n})]\right\}+\frac{3}{2}\left(r_{2}-1\right) s_{0}(\underline{\underline{n n}}-\underline{\underline{\delta}} / 3)(\underline{\underline{\sigma}}: \underline{n n}) \\
& \left.\left.=G_{0} \underline{\underline{e}}+\hat{a}_{1}[\underline{\underline{n}} \cdot \underline{\underline{e}}+\underline{\underline{e}} \cdot \underline{\underline{n}}-2 \underline{n} \underline{\underline{e}}: \underline{\underline{n n}})\right]+(\hat{\beta}-3 / 2)(\underline{\underline{n}}-\underline{\underline{\delta}} / 3)(\underline{\underline{e}}: \underline{n n})\right\}
\end{aligned}
$$

Since $\theta_{0}=\frac{\eta_{0}}{G_{0}}=\frac{1}{s_{0}}$, equation (4-29) can be rewritten as

$$
\begin{aligned}
& \theta_{0}(\underline{\underline{\sigma}} t+\underline{\underline{\sigma}} \cdot \underline{\underline{\omega}}+\underline{\underline{\omega}} \cdot \underline{\underline{\sigma}})+\underline{\underline{\sigma}}+\left(r_{1}-1\right)[\underline{\underline{n n}} \cdot \underline{\underline{\sigma}}+\underline{\underline{\sigma}} \cdot \underline{n n}-2 \underline{n n}(\underline{\underline{\sigma}}: \underline{n n})]+\frac{3}{2}\left(r_{2}-1\right)(\underline{n n}-\underline{\underline{\delta}} / 3)(\underline{\underline{\sigma}}: \underline{n n}) \\
& =\eta_{0}\left\{\underline{\underline{e}}+\hat{a}_{1}[\underline{n n} \cdot \underline{\underline{e}}+\underline{\underline{e}} \cdot \underline{n n}-2 \underline{n n}(\underline{\underline{e}}: \underline{n n})]+(\hat{\beta}-3 / 2)(\underline{n n}-\underline{\underline{\delta}} / 3)(\underline{\underline{e}}: \underline{n n})\right\}
\end{aligned}
$$

\subsection{Equations of Weak Maxwell Type Nematodynamics in Simple Shearing}

Under simple shearing, the analytical solution of weakly nonlinear viscoelastic nematodynamic constitutive equations can be simplified. The shearing flows is analyzed utilizing the common Cartesian coordinate system $\{\underline{x}\}=\left\{x_{1}, x_{2}, x_{3}\right\}$ where $x_{1}$ is directed along the flow and $x_{2}$ along the velocity gradient. In this coordinate system, the tensors of strain rate $\underset{\underline{e}}{(}(t)$ and vorticity $\underline{\underline{\omega}}(t)$ for homogeneous shearing flows have the matrix forms:

$$
\underline{\underline{e}}(t)=\dot{\gamma}(t) \underline{\underline{\alpha}} \quad \underline{\underline{\omega}}(t)=\dot{\gamma}(t) \underline{\underline{\beta}}, \underline{\underline{\alpha}}=\frac{1}{2}\left(\begin{array}{lll}
0 & 1 & 0 \\
1 & 0 & 0 \\
0 & 0 & 0
\end{array}\right), \underline{\underline{\beta}}=\frac{1}{2}\left(\begin{array}{ccc}
0 & -1 & 0 \\
1 & 0 & 0 \\
0 & 0 & 0
\end{array}\right)
$$




\subsubsection{Evolution of Director Located in the $\left\{\mathrm{x}_{1}, \mathrm{x}_{2}\right\}$ Shear Plane}

Substituting expression for $n=\left\{n_{1}, n_{2}, 0\right\}$ and kinematical matrices (4-31) into

evolution equation for director (4-23) yields the evolution equation for the longitudinal component $n_{1}$ of director.

$$
\begin{aligned}
& \theta *\left(\ddot{n}_{1}+n_{1} \dot{n}_{1}^{2} / n_{2}^{2}\right)+\dot{n}_{1}-\dot{\gamma} n_{2} / 2=\theta * \ddot{\gamma}\left[\lambda_{e}\left(1-2 n_{1}^{2}\right)+1\right] n_{2} / 2 \\
& +\lambda_{e} \theta^{*} \dot{\gamma}^{2} n_{1} n_{2}^{2}+\lambda_{v} \dot{\gamma} n_{2}\left(1-2 n_{1}^{2}\right) / 2
\end{aligned}
$$

Here $n_{2}=\sqrt{1-n_{1}^{2}}$.

\subsubsection{Formula for Director in Steady Shearing of Aligning Nematics}

In the case of aligning nematics in steady shearing, $\dot{\gamma}=$ constant and $n_{1}=$ constant, which means that $\ddot{\gamma}=0, \dot{n}_{1}=0$, and $\ddot{n}_{1}=0$. Then, eqution (4-32) can be transformed to:

$$
n_{2}\left(1+2 \lambda_{e} D n_{1} n_{2}+\lambda_{v}\left(1-2 n_{1}^{2}\right)\right)=0
$$

Here $D=\dot{\gamma} \theta^{*}$ is the flow Deborah number. Equation (4-33) is satisfied in two cases:

either for $n_{2}=0$ or $\left(1+2 \lambda_{e} D n_{1} n_{2}+\lambda_{v}\left(1-2 n_{1}^{2}\right)\right)=0$.

If $n_{2}=0, n_{1}= \pm 1$, which means the completely aligning case. If $n_{2} \neq 0$, then

$\left(1+2 \lambda_{e} D n_{1} n_{2}+\lambda_{v}\left(1-2 n_{1}{ }^{2}\right)\right)=0$. The solution of this equation is:

$$
n_{1}{ }^{2}=\frac{1}{2}\left(1+\lambda_{v} \frac{1+\left|D \lambda_{e} / \lambda_{v}\right| \sqrt{D^{2} \lambda_{e}{ }^{2}+\lambda_{v}{ }^{2}-1}}{D^{2} \lambda_{e}{ }^{2}+\lambda_{v}{ }^{2}}\right)
$$

There are evident simple solutions of (4-34) for particular cases of viscous tumbling parameter $\lambda_{v}: n_{1}= \pm 1$ when $\lambda_{v}=1$, and $n_{1}=0$ when $\lambda_{v}=-1$. Thus formula (4-34) covers both the limiting solutions of the second term in (4-33), $n_{1}=0$ and $n_{1}= \pm 1$, when 
$\lambda_{v} \rightarrow \pm 1$, respectively. Imposing the condition $0 \leq n_{1}{ }^{2} \leq 1$ results in the criterion of existence of steady solution for aligning case:

$$
r^{2}=D^{2} \lambda_{e}^{2}+\lambda_{v}^{2} \geq 1
$$

By using $D \lambda_{e}=r \cos \psi, \lambda_{v}=r \sin \psi, r^{-1}=\cos \phi$, one can prove that the second term in bracket in (4-34) does not exceed the unity:

$$
\lambda_{v} \frac{1+\left|D \lambda_{e} / \lambda_{v}\right| \sqrt{D^{2} \lambda_{e}{ }^{2}+\lambda_{v}{ }^{2}-1}}{D^{2} \lambda_{e}{ }^{2}+\lambda_{v}{ }^{2}}=\cos \phi \sin \psi \pm \sin \phi \cos \psi=\sin (\psi \pm \phi)
$$

Here \pm corresponds to the \pm signs of $\lambda_{v}$.

Formulas (4-34) and (4-35) show that in very slow flow when $D<<1$, the criterion of occurrence of aligning case coincides with that known for viscous (low molecular weight) nematic fluids. Nevertheless at higher values of $\mathrm{D}$, the model predicts the existing of aligning flow situation even if $\left|\lambda_{v}\right|<1$. But the present weakly nonlinear theory is uncapable to describe the strong viscoelastic nematic flows with high values of D. The non-aligning case, $r^{2}<1$ is beyond the scope of our investigation.

\subsubsection{Evolution of Director}

Substituting $n_{1}=\cos \varphi, \quad n_{2}=\sin \varphi$ into equation (4-32), yields:

$$
\theta^{*} \ddot{\varphi}+\dot{\varphi}+\dot{\gamma} / 2+\left[\lambda_{e} \theta * \ddot{\gamma}\left(1-2 n_{1}{ }^{2}\right)+\theta * \ddot{\gamma}\right] n_{2} / 2+\lambda_{e} \theta^{*} \dot{\gamma}^{2} n_{1} n_{2}+\lambda_{v} \dot{\gamma}\left(1-2 n_{1}{ }^{2}\right) / 2=0
$$

Equation (4.37) yields the following condition for step variations of derivative $\dot{\varphi}$, caused by the step variation of shear rate:

$$
\dot{\varphi}_{+}-\dot{\varphi}_{-}+\left(\dot{\gamma}_{+}-\dot{\gamma}_{-}\right)\left[\lambda_{e}\left(1-2 n_{1}{ }^{2}\right)+1\right] / 2=0
$$


Here $n_{1}$ is continuous value of $x$-projection of director at the step instance $t=t_{0}$, whereas $\dot{\varphi}_{ \pm}=\dot{\varphi}\left(t_{0} \pm 0\right)$, and $\dot{\gamma}_{ \pm}=\dot{\gamma}\left(t_{0} \pm 0\right)$. We now apply relations (4-38) to two important cases of shearing deformations of viscous aligning nematics.

(a) Evolution of director after cessation of steady shearing: $\dot{\gamma}=0$ at $t>0$. Here for steady flow, $\dot{\gamma}_{+}=0, \dot{\gamma}_{-}=\dot{\gamma}_{0}$, and $\dot{\varphi}_{+}=\dot{\varphi}(t+0)=\dot{\varphi}_{0}, \dot{\varphi}_{-}=0$. Then equation (4-38) can be rewritten as:

$$
\dot{\varphi}_{0}=\dot{\gamma}_{0}\left[\lambda_{e}\left(1-2 n_{1,0}{ }^{2}\right)+1\right] / 2
$$

Here $n_{1,0}$ is the value of director in the steady shearing flow. In this case $\dot{\gamma}=0$ for $t>0$, and equation (4-37) reduces to the relaxation equation of director:

$$
\theta^{*} \ddot{\varphi}+\dot{\varphi}=0
$$

Solution of this equation is:

$$
\varphi(t)=\varphi_{0}+\dot{\varphi}_{0} \theta^{*}\left(1-e^{-t / \theta^{*}}\right)
$$

Here $\varphi_{0}=\arccos \left(n_{1,0}(\dot{\gamma})\right)$ corresponds to the steady shearing, $\dot{\varphi}_{0}$ is described by equation (4-39) where $n_{1,0}=\cos \varphi_{0}$. In the limit $t \rightarrow \infty$, formula (4.41) gives the value of $\varphi_{r}$ describing the position of director after relaxation, i.e. at rest:

$$
\varphi_{r}=\varphi_{0}+\theta * \dot{\gamma}_{0}\left[\lambda_{e}\left(1-2 n^{2}{ }_{1,0}\right)+1\right] / 2
$$

Then, the value of director after relaxation can be calculated as $n_{1 r}=\cos \varphi_{r}$.

(b) The evolution of director in start up flow from the rest state: In the case of start-up, $\dot{\gamma}_{+}=\dot{\gamma}_{0} ; \dot{\gamma}_{-}=0 ; \dot{\varphi}_{+}=\dot{\varphi}(t+0)=\dot{\varphi}_{0} ; \dot{\varphi}_{-}=0$. So equation (4-38) can be presented as:

$$
\dot{\varphi}_{0}=-\dot{\gamma}_{0}\left[\lambda_{e}\left(1-2 n_{1 r}{ }^{2}\right)+1\right] / 2
$$

When $\mathrm{t}>0$, equation (4-32) becomes: 


$$
\theta *\left(\ddot{n}_{1}+n_{1} \dot{n}_{1}^{2} / n_{2}{ }^{2}\right)+\dot{n}_{1}=\dot{\gamma}_{0} n_{2} / 2+\lambda_{e} \theta * \dot{\gamma}_{0}{ }^{2} n_{1} n_{2}{ }^{2}+\lambda_{v} \dot{\gamma}_{0} n_{2}\left(1-2 n_{1}{ }^{2}\right) / 2
$$

Using evident relation, $\dot{n}_{1 r}=-n_{2 r} \dot{\varphi}_{0}$, one can obtain the initial conditions for equation (4-44):

$$
\left.n_{1}\right|_{t=0}=n_{1 r}=\cos [\varphi(\infty)] ;\left.\dot{n}_{1}\right|_{t=0}=n_{2 r} \dot{\gamma}_{0}\left[\lambda_{e}\left(1-2 n_{1 r}{ }^{2}\right)+1\right] / 2
$$

Solving equation (4-44) with the initial conditions (equation (4-45)), one can obtain the numerical solution for director $n_{1}$ in start up flow.

\subsubsection{Unsteady Shearing of Aligning Viscoelastic Nematics}

In steady shearing flow, the matrices of stress $\underline{\underline{\sigma}}$, vorticity $\underline{\underline{\omega}}$, strain rate $\underline{\underline{e}}$, director $\underline{n}$, dyadic $\underline{n} \underline{n}$, and the unit tensor $\underline{\underline{\delta}}$ are given as follows:

$$
\begin{aligned}
& \underline{\underline{\sigma}}=\left(\begin{array}{ccc}
\sigma_{11} & \sigma_{12} & 0 \\
\sigma_{12} & \sigma_{22} & 0 \\
0 & 0 & \sigma_{33}
\end{array}\right) ; \quad \underline{\underline{\omega}}=\frac{\dot{\gamma}}{2}\left(\begin{array}{ccc}
0 & -1 & 0 \\
1 & 0 & 0 \\
0 & 0 & 0
\end{array}\right) ; \quad \underline{\underline{e}}=\frac{\dot{\gamma}}{2}\left(\begin{array}{ccc}
0 & 1 & 0 \\
1 & 0 & 0 \\
0 & 0 & 0
\end{array}\right) ; \quad \underline{n}=\left(\begin{array}{c}
n_{1} \\
n_{2} \\
0
\end{array}\right) \\
& \underline{n n}=\left(\begin{array}{ccc}
n_{1}^{2} & n_{1} n_{2} & 0 \\
n_{1} n_{2} & n_{2}^{2} & 0 \\
0 & 0 & 0
\end{array}\right) ; \quad \underline{\underline{\delta}}=\left(\begin{array}{ccc}
1 & 0 & 0 \\
0 & 1 & 0 \\
0 & 0 & 1
\end{array}\right)
\end{aligned}
$$

After easy calculations equation (4-30) can be rewritten in the matrix form as:

$$
\begin{gathered}
\theta_{0}\left(\begin{array}{ccc}
\dot{\sigma}_{11} & \dot{\sigma}_{12} & 0 \\
\dot{\sigma}_{12} & \dot{\sigma}_{22} & 0 \\
0 & 0 & \dot{\sigma}_{33}
\end{array}\right)+\theta_{0} \dot{\gamma}\left(\begin{array}{ccc}
-\sigma_{12} & \frac{\sigma_{11}-\sigma_{22}}{2} & 0 \\
\frac{\sigma_{11}-\sigma_{22}}{2} & \sigma_{12} & 0 \\
0 & 0 & 0
\end{array}\right)+\left(\begin{array}{ccc}
\sigma_{11} & \sigma_{12} & 0 \\
\sigma_{12} & \sigma_{22} & 0 \\
0 & 0 & \sigma_{33}
\end{array}\right)+ \\
\left(r_{1}-1\right)\left(\begin{array}{ccc}
2 n_{1}^{2} \sigma_{11}+2 n_{1} n_{2} \sigma_{12} & \sigma_{12}+n_{1} n_{2}\left(\sigma_{11}+\sigma_{22}\right) & 0 \\
\sigma_{12}+n_{1} n_{2}\left(\sigma_{11}+\sigma_{22}\right) & 2 n_{2}^{2} \sigma_{22}+2 n_{1} n_{2} \sigma_{12} & 0 \\
0 & 0 & 0
\end{array}\right)+
\end{gathered}
$$




$$
\begin{aligned}
& {\left[-2\left(r_{1}-1\right)\left(\begin{array}{ccc}
n_{1}^{2} & n_{1} n_{2} & 0 \\
n_{1} n_{2} & n_{2}^{2} & 0 \\
0 & 0 & 0
\end{array}\right)+\frac{3}{2}\left(r_{2}-1\right)\left(\begin{array}{ccc}
n_{1}{ }^{2}-\frac{1}{3} & n_{1} n_{2} & 0 \\
n_{1} n_{2} & n_{2}{ }^{2}-\frac{1}{3} & 0 \\
0 & 0 & -\frac{1}{3}
\end{array}\right)\right]\left(n_{1}^{2} \sigma_{11}+n_{2}{ }^{2} \sigma_{22}+2 n_{1} n_{2} \sigma_{12}\right)=} \\
& \eta_{0} \dot{\gamma}\left(\begin{array}{ccc}
\hat{a}_{1} n_{1} n_{2}-2 \hat{a}_{1} n_{1} n_{2}+\left(\hat{\beta}-\frac{3}{2}\right)\left(n_{1}{ }^{2}-\frac{1}{3}\right) n_{1} n_{2} & \frac{1}{2}+\frac{\hat{a}_{1}}{2}-2 \hat{a}_{1} n_{1}{ }^{2} n_{2}{ }^{2}+\left(\hat{\beta}-\frac{3}{2}\right) n_{1}{ }^{2} n_{2}{ }^{2} & 0 \\
\frac{1}{2}+\frac{\hat{a}_{1}}{2}-2 \hat{a}_{1} n_{1}{ }^{2} n_{2}{ }^{2}+\left(\hat{\beta}-\frac{3}{2}\right) n_{1}{ }^{2} n_{2}{ }^{2} & \hat{a}_{1} n_{1} n_{2}-2 \hat{a}_{1} n_{1} n_{2}{ }^{3}+\left(\hat{\beta}-\frac{3}{2}\right)\left(n_{2}{ }^{2}-\frac{1}{3}\right) n_{1} n_{2} & 0 \\
0 & 0 & -\frac{1}{3}\left(\hat{\beta}-\frac{3}{2}\right) n_{1} n_{2}
\end{array}\right)
\end{aligned}
$$

This matrix equation yields three independent differential equations:

$$
\begin{aligned}
& \theta_{0} \dot{\sigma}_{12}+\theta_{0} \dot{\gamma} \frac{\sigma_{11}-\sigma_{22}}{2}+\sigma_{12}+\left(r_{1}-1\right)\left[\sigma_{12}+n_{1} n_{2}\left(\sigma_{11}+\sigma_{22}\right)\right]+\left(\sigma_{11} n_{1}{ }^{2}+\sigma_{22} n_{2}{ }^{2}+2 \sigma_{12} n_{1} n_{2}\right) \\
& n_{1} n_{2}\left[\frac{3}{2}\left(r_{2}-1\right)-2\left(r_{1}-1\right)\right]=\frac{\eta_{0} \dot{\gamma}}{2}\left(1+\hat{a}_{1}+2 n_{1}{ }^{2} n_{2}{ }^{2}\left(\hat{\beta}-\frac{3}{2}-2 \hat{a}_{1}\right)\right)
\end{aligned}
$$

$$
\begin{aligned}
& \theta_{0} \dot{\sigma}_{11}-\sigma_{12} \theta_{0} \dot{\gamma}+\sigma_{11}+2\left(r_{1}-1\right)\left(\sigma_{11} n_{1}^{2}+\sigma_{12} n_{1} n_{2}\right)+\left[\frac{3}{2}\left(r_{2}-1\right)\left(n_{1}^{2}-\frac{1}{3}\right)-2\left(r_{1}-1\right) n_{1}^{2}\right] \\
& \times\left(\sigma_{11} n_{1}^{2}+\sigma_{22} n_{2}^{2}+2 \sigma_{12} n_{1} n_{2}\right)=\eta_{0} \dot{m} n_{1} n_{2}\left(\hat{a}_{1}\left(1-2 n_{1}^{2}\right)+\left(\hat{\beta}-\frac{3}{2}\right) \times\left(n_{1}^{2}-\frac{1}{3}\right)\right)
\end{aligned}
$$

$$
\begin{aligned}
& \theta_{0} \dot{\sigma}_{22}+\sigma_{12} \theta_{0} \dot{\gamma}+\sigma_{22}+2\left(r_{1}-1\right)\left(\sigma_{22} n_{2}{ }^{2}+\sigma_{12} n_{1} n_{2}\right)+\left[\frac{3}{2}\left(r_{2}-1\right)\left(n_{2}{ }^{2}-\frac{1}{3}\right)-2\left(r_{1}-1\right) n_{2}{ }^{2}\right] \\
& \times\left(\sigma_{11} n_{1}^{2}+\sigma_{22} n_{2}{ }^{2}+2 \sigma_{12} n_{1} n_{2}\right)=\eta_{0} \dot{m} n_{1} n_{2}\left(\hat{a}_{1}\left(1-2 n_{2}{ }^{2}\right)+\left(\hat{\beta}-\frac{3}{2}\right)\left(n_{2}{ }^{2}-\frac{1}{3}\right)\right)
\end{aligned}
$$

Equations (4-46a-c) describe the evolution and/or steady state behavior of stress tensor. In case of steady shear flow, the value of director can be explicitely presented by equation (4-34). The value of director after relaxation can be calculated as $n_{1 r}=\cos \varphi_{r}$ using equation (4-42), and the evolution of director in start up flow from the rest state can 
be obtained from equation (4-44) and (4-45). Solutions of these equations in comparison with experimental data are considered in the next chapter. 


\section{CHAPTER V}

\section{RESULTS AND DISCUSSION}

\subsection{Introduction}

Modeling of rheological properties of liquid crystalline polymers has encountered a lot of problems when using either continuum or molecular theories. These problems are mostly related to a large number of unknown parameters in the model equations. We use in our simulations below a recently developed new model of weak viscoelastic nematodynamics [12-14], based on the assumption of smallness of elastic (transient) strains and elastic relative rotation. The model constitutive equations combine the de Gennes-type elastic potential and LEP viscous nematic approach, while ignoring Frank elasticity and inertia effects. In the absence of magnetic field, the theory has viscoelastic transversally anisotropic properties when the stress tensor is symmetric and orientation of director is only caused by flow. Thus, this simplified approach has led to a closed set of two coupled anisotropic viscoelastic equations for evolution of director and extra stress. It should also be mentioned that it is possible to obtain analytic solutions of nematodynamic equations for steady simple shearing flow.

Based on the simplified nematodynamic model, we will numerically study the basic flows of polymer aligning nematics in shearing flows. In our simulations, we determine the constitutive parameters in the model by solving the constitutive equations with the use of "Mathematica" software and curve fitting of the existing experimental 
data with Matlab. The descriptive ability of this theory will compared with various literature experimental data describing the rheological properties of liquid crystalline polymers.

\subsection{Simulation Procedures}

In order to test the reliability of constitutive equations of weak viscoelastic nematodynamics, we compare the results of numerical simulations with published rheological experimental data $[59,63]$. The ultimate goal of simulations was to explore the very possibility to describe experimental data while determining the constitutive parameters with exostive fitting procedures under different conditions, including steady shear flows, relaxation, and transient start-up flows.

\subsubsection{Determining Constitutive Parameters from Steady Shear Flows}

In steady simple shear flow $\partial \sigma / \partial t=0$. Therefore, equation (4-46.a-c) can be rewritten as:

$$
\begin{aligned}
& \theta_{0} \dot{\gamma} \frac{\sigma_{11}-\sigma_{22}}{2}+\sigma_{12}+\left(r_{1}-1\right)\left[\sigma_{12}+n_{1} n_{2}\left(\sigma_{11}+\sigma_{22}\right)\right]+\left(\sigma_{11} n_{1}{ }^{2}+\sigma_{22} n_{2}{ }^{2}+2 \sigma_{12} n_{1} n_{2}\right) \\
& n_{1} n_{2}\left[\frac{3}{2}\left(r_{2}-1\right)-2\left(r_{1}-1\right)\right]=\frac{\eta_{0} \dot{\gamma}}{2}\left(1+\hat{a}_{1}+2 n_{1}{ }^{2} n_{2}{ }^{2}\left(\hat{\beta}-\frac{3}{2}-2 \hat{a}_{1}\right)\right) \\
& -\sigma_{12} \theta_{0} \dot{\gamma}+\sigma_{11}+2\left(r_{1}-1\right)\left(\sigma_{11} n_{1}^{2}+\sigma_{12} n_{1} n_{2}\right)+\left[\frac{3}{2}\left(r_{2}-1\right)\left(n_{1}^{2}-\frac{1}{3}\right)-2\left(r_{1}-1\right) n_{1}^{2}\right] \\
& \times\left(\sigma_{11} n_{1}^{2}+\sigma_{22} n_{2}^{2}+2 \sigma_{12} n_{1} n_{2}\right)=\eta_{0} \dot{m}_{1} n_{2}\left(\hat{a}_{1}\left(1-2 n_{1}^{2}\right)+\left(\hat{\beta}-\frac{3}{2}\right) \times\left(n_{1}^{2}-\frac{1}{3}\right)\right) \\
& \sigma_{12} \theta_{0} \dot{\gamma}+\sigma_{22}+2\left(r_{1}-1\right)\left(\sigma_{22} n_{2}{ }^{2}+\sigma_{12} n_{1} n_{2}\right)+\left[\frac{3}{2}\left(r_{2}-1\right)\left(n_{2}{ }^{2}-\frac{1}{3}\right)-2\left(r_{1}-1\right) n_{2}{ }^{2}\right] \\
& \times\left(\sigma_{11} n_{1}^{2}+\sigma_{22} n_{2}^{2}+2 \sigma_{12} n_{1} n_{2}\right)=\eta_{0} \dot{m} n_{1} n_{2}\left(\hat{a}_{1}\left(1-2 n_{2}^{2}\right)+\left(\hat{\beta}-\frac{3}{2}\right)\left(n_{2}^{2}-\frac{1}{3}\right)\right)
\end{aligned}
$$


Equations (5-1.a-c) were analytically solved by "Mathematica", and then $\sigma_{11}, \sigma_{12}$, and $\sigma_{22}$ were expressed as functions of shear rate $\dot{\gamma}$ with values of the constitutive parameters, $\theta_{0}, r_{1}, r_{2}, \lambda_{e}, \lambda_{v}, \eta_{0}, \hat{a}_{1}$, and $\hat{\beta}$ (see Appendix A-1 for detail) yet to be determined. Equations (5-1.a-c) hold the identity $\sigma_{33}=-\sigma_{11}-\sigma_{22}$. Thus the first and second normal stress differences have been introduced in standard way as: $N_{1}=\sigma_{11}-\sigma_{22}, \quad N_{2}=\sigma_{22}-\sigma_{33}$.

We then employed "Matlab" program to find the constitutive parameters by curve fitting with the steady shear flows from the experimental data for non-Newtonian viscosity $\eta$ and $\mathrm{N}_{1}$ as functions of $\dot{\gamma}$. Using relation (4-34), the projection $n_{1}$ of director in the flow direction was presented as:

$$
n_{1}=\sqrt{\frac{1}{2}\left(1+\lambda_{v} \frac{1+\left|D \lambda_{e} / \lambda_{v}\right| \sqrt{D^{2} \lambda_{e}{ }^{2}+\lambda_{v}{ }^{2}-1}}{D^{2} \lambda_{e}{ }^{2}+\lambda_{v}{ }^{2}}\right)}
$$

Here $D=\dot{\gamma} \theta^{*}$ is the flow Debroah number, where $\theta^{*}=\theta_{0} / r_{1}$. The component of director $n_{2}$ in direction orthogonal to flow was presented as:

$$
n_{2}=\sqrt{1-n_{1}^{2}}
$$

Given the values of tumbling parameters $\lambda_{\mathrm{v}}$ and $\lambda_{e}$ and the values $\theta_{0}$ and $r_{1}$, the director projections $n_{1}$ and $n_{2}$ could be calculated for any given value of shear rate $\dot{\gamma}$.

For a chosen set of parameters of $\theta_{0}, r_{1}, r_{2}, \lambda_{e}, \lambda_{v}, \eta_{0}, \hat{a}_{1}$, and $\hat{\beta}$, we calculated values of $\eta$ and $\mathrm{N}_{1}$ for a given $\dot{\gamma}$ using the expressions obtained by "Mathematica", which were compared with the corresponding experimental data of $\eta$ and $N_{1}$. If there was 
a big difference between the experimental data and simulated results, the above parameters have to be changed. Recalculations were continued till a good enough agreement between experimental values of $\eta$ and $N_{1}$ and simulated results was achieved.

\subsubsection{Simulation of Relaxation Data}

During the relaxation process, $\dot{\gamma}=0$. Therefore, equations (4-46.a-c) can be rewritten as:

$$
\begin{aligned}
& \theta_{0} \dot{\sigma}_{12}+\sigma_{12}+\left(r_{1}-1\right)\left[\sigma_{12}+n_{1} n_{2}\left(\sigma_{11}+\sigma_{22}\right)\right] \\
& +\left(\sigma_{11} n_{1}{ }^{2}+\sigma_{22} n_{2}{ }^{2}+2 \sigma_{12} n_{1} n_{2}\right) n_{1} n_{2}\left[\frac{3}{2}\left(r_{2}-1\right)-2\left(r_{1}-1\right)\right]=0 \\
& \theta_{0} \dot{\sigma}_{11}+\sigma_{11}+2\left(r_{1}-1\right)\left(\sigma_{11} n_{1}{ }^{2}+\sigma_{12} n_{1} n_{2}\right)+ \\
& {\left[\frac{3}{2}\left(r_{2}-1\right)\left(n_{1}{ }^{2}-\frac{1}{3}\right)-2\left(r_{1}-1\right) n_{1}{ }^{2}\right] \times\left(\sigma_{11} n_{1}{ }^{2}+\sigma_{22} n_{2}{ }^{2}+2 \sigma_{12} n_{1} n_{2}\right)=0} \\
& \theta_{0} \dot{\sigma}_{22}+\sigma_{22}+2\left(r_{1}-1\right)\left(\sigma_{22} n_{2}{ }^{2}+\sigma_{12} n_{1} n_{2}\right)+ \\
& {\left[\frac{3}{2}\left(r_{2}-1\right)\left(n_{2}{ }^{2}-\frac{1}{3}\right)-2\left(r_{1}-1\right) n_{2}{ }^{2}\right] \times\left(\sigma_{11} n_{1}{ }^{2}+\sigma_{22} n_{2}{ }^{2}+2 \sigma_{12} n_{1} n_{2}\right)=0} \\
& \left.{ }^{2}\right)=0
\end{aligned}
$$

Here $n_{1}=\cos \varphi(t)$, and $n_{2}=\sin \varphi(t)$.

Due to equation (4-42) $\varphi(t)=\varphi_{0}+\dot{\gamma}_{0} \theta^{*}\left[\lambda_{e}\left(1-2 n^{2}{ }_{1,0}\right)+1\right] / 2\left(1-e^{\frac{-t}{\theta^{*}}}\right)$, where $n_{1,0}$ is the value of director in steady shearing (see Section 5.2.1), and $\varphi_{0}=\arccos \left(n_{1,0}(\dot{\gamma})\right)$. Using the intial values of $\sigma_{11}(0), \sigma_{12}(0)$, and $\sigma_{22}(0)$ obtained from steady shear flow as well as the determined set of $\theta_{0}, r_{1}, r_{2}, \lambda_{e}, \lambda_{v}, \eta_{0}, \hat{a}_{1}$, and $\hat{\beta}$, equations (5-4.a-c) were solved numerically with "Mathematica". The results were compared with experimental data. If the simulated results were significantly different from experimental data, we went 
back to the previous step described in Section 5.2.1 to re-determine the constitutive parameters as well as $n_{1,0}, \sigma_{11}(0), \sigma_{12}(0)$, and $\sigma_{22}(0)$. Recalculation would continue till good results of simulated relaxation were achieved.

\subsubsection{Simulation of Transient Start-up Shear Flow}

For a determined set of $\theta_{0}, r_{1}, r_{2}, \lambda_{e}, \lambda_{v}, \eta_{0}, \hat{a}_{1}$, and $\hat{\beta}$, we solved numerically equations (4-46.a-c) to obtain values of shear stress $\left(\sigma_{12}\right)$ and first normal stress difference $\left(\mathrm{N}_{1}\right)$ in start-up shearing flow with a given $\dot{\gamma}$. If the calculated $\sigma_{12}$ and $\mathrm{N}_{1}$ deviated far away from their respective experimental data, we went back to Section 5.2.1 to re-determine $\theta_{0}, r_{1}, r_{2}, \lambda_{e}, \lambda_{v}, \eta_{0}, \hat{a}_{1}$, and $\hat{\beta}$, and also to Section 5.2.2 to recalculate the relaxation data, and then simulate the transient start-up flow once again. This iterative procedure continued till the simulated results of steady shear flow, relaxation, and transient flow were all close to experimental data for a given set of $\theta_{0}, r_{1}$, $r_{2}, \lambda_{e}, \lambda_{v}, \eta_{0}, \hat{a}_{1}$, and $\hat{\beta}$

\subsection{Choice of Parameters for Simulation}

The best choice of constitutive parameters for simulating the experimental data of Titan at $340{ }^{\circ} \mathrm{C}$ are given as follows: $\hat{a}_{1}=0.2, r_{1}=0.15, r_{2}=0.222, \lambda_{e}=2.2, \lambda_{v}=30$, $\hat{\beta}=0.5, \theta_{0}=0.3 \mathrm{sec}$, and $\eta_{0}=0.34 \mathrm{kPa} \cdot \mathrm{sec}$. For Zenite 6000 at $360{ }^{\circ} \mathrm{C}$, the chosen parameters are: $\hat{a}_{1}=4, r_{1}=0.55, r_{2}=0.367, \lambda_{e}=1.01, \lambda_{v}=50, \hat{\beta}=0.1, \theta_{0}=2.5$ sec, and $\eta_{0}=1.55 \mathrm{kPa} \cdot \mathrm{sec}$. We remind that Titan is random copolyester of ethyleneterephthalate (PET) and hydroxybenzoic acid (HNA) with two methylene flexible spacers, 
whereas Zenite 6000 is a fully aromatic copolyester with kinks. Therefore, Zenite 6000 is expected to be much more rigid than Titan. Our simulations confirmed this, with the relaxation time $\theta_{0}$, the viscous "tumbling" parameter $\lambda_{v}, \eta_{0}, \hat{a}_{1}, r_{1}$, and $r_{2}$ of Titan being smaller than those of Zenite 6000, while the elastic "tumbling" parameter $\lambda_{e}$ and $\hat{\beta}$ of Titan are greater than those of Zenite 6000 .

The best constitutive parameters of simulating the experimental data of PSHQ9 at 130, 140 and $150{ }^{\circ} \mathrm{C}$ are presented in Table 5.1. For different temperatures, $r_{1}, r_{2}, \lambda_{e}$, $\lambda_{v}, \hat{a}_{1}$, and $\hat{\beta}$ of PSHQ9 are kept constant, whereas $\theta_{0}$ and $\eta_{0}$ vary with the increased temperatures. This trend for $\theta_{0}$ and $\eta_{0}$ was expected.

The best constitutive parameters found from simulating the experimental data of PI-14-5CN at 70, 80 and $90{ }^{\circ} \mathrm{C}$ are given in Table 5.2, where $r_{1}, r_{2}, \lambda_{e}, \lambda_{v}, \hat{a}_{1}$, and $\hat{\beta}$ remain unchanged while $\theta_{0}$ and $\eta_{0}$ reduced with the increased temperature.

Table 5.1 The constitutive parameters of simulating the experimental data of PSHQ9 at 130,140 and $150{ }^{\circ} \mathrm{C}$

\begin{tabular}{|c|c|c|c|}
\hline Temperature & $130{ }^{\circ} \mathrm{C}$ & $140{ }^{\circ} \mathrm{C}$ & $150{ }^{\circ} \mathrm{C}$ \\
\hline$\hat{a}_{1}$ & 5 & 5 & 5 \\
\hline$r_{1}$ & 0.7 & 0.7 & 0.7 \\
\hline$r_{2}$ & 0.533 & 0.533 & 0.533 \\
\hline$\lambda_{e}$ & 2 & 2 & 2 \\
\hline$\lambda_{v}$ & 18 & 18 & 18 \\
\hline$\hat{\beta}$ & 8 & 8 & 2.2 \\
\hline$\theta_{0}(\mathrm{sec})$ & 3 & 2.8 & 10 \\
\hline$\eta_{0}(\mathrm{kPa} \cdot \mathrm{sec})$ & 220 & 70 & \\
\hline
\end{tabular}


Table 5.2 The constitutive parameters of simulating the experimental data of PI-14-5CN at 70,80 and $90{ }^{\circ} \mathrm{C}$

\begin{tabular}{|c|c|c|c|}
\hline Temperature & $70{ }^{\circ} \mathrm{C}$ & $80{ }^{\circ} \mathrm{C}$ & $90{ }^{\circ} \mathrm{C}$ \\
\hline$\hat{a}_{1}$ & 0.7 & 0.7 & 0.7 \\
\hline$r_{1}$ & 0.5 & 0.5 & 0.5 \\
\hline$r_{2}$ & 0.9 & 0.9 & 0.9 \\
\hline$\lambda_{e}$ & 2 & 2 & 2 \\
\hline$\lambda_{v}$ & 12 & 12 & 12 \\
\hline$\hat{\beta}$ & 1.3 & 1.3 & 1.3 \\
\hline$\theta_{0}(\mathrm{sec})$ & 1.5 & 0.8 & 0.28 \\
\hline$\eta_{0}(\mathrm{kPa} \cdot \mathrm{sec})$ & 200 & 9 & 2.9 \\
\hline
\end{tabular}

As compared with Table 5.1, it can be found out that all the constitutive parameters except for $r_{2}$ and $\lambda_{e}$ of PSHQ9 are larger than those of PI-14-5CN. We remind that PSHQ9 is a main-chain LCP, whereas PI-14-5CN is a side-chain LCP. The flexible main chain in PI-14-5CN makes it much easier to relax and decouple the mesogen from main chain. In contrast, the main-chain LCP (PSHQ9) possesses mesogens in the main chain, which are tightly packed together and in turn have relatively low mobility and capability of relaxation.

\subsection{Simulated Results for Steady Shearing Flow}

Figure 5.1 presents plots of experimental data of viscosity $\eta$, shear stress $\sigma_{12}$, and first normal stress difference $\mathrm{N}_{1}$ versus shear rates $\dot{\gamma}$ for Titan at $340{ }^{\circ} \mathrm{C}$, with the parameters found by mentioned curve fitting procedure. There is a good agreement between simulations and experimental data. But, the fitting curve for logarithmic plot of $\eta$ versus $\dot{\gamma}$ does not exhibit the three region flow curve [64], probably due to the fact that 
only a narrow part of region II presents in the experimental data. The region I should appear at very low shear rates $\dot{\gamma}$, if there is a texture which the current monodomain approach is uncapable to describe. It should be noted that the first normal stress difference is positive in the range of shear rates investigated.

Figure 5.2 shows plot of viscosity $\eta$ versus shear rates $\dot{\gamma}$ for Zenite 6000 at $360{ }^{\circ} \mathrm{C}$. Because of the limited number of experimental data, we can only obtain a relatively linear fitting curve. The region I occurs at higher shear rate (say below $1.5 \mathrm{~s}^{-1}$ ) for Zenite 6000 due to its higher rigidity, as compared to Titan. The region III will appear as the shear rate increases to a certain value.

Figure 5.3 presents the logarithmic plots of experimental data of $\eta$ vs $\dot{\gamma}$ (open symbols) and $\mathrm{N}_{1}$ vs $\dot{\gamma}$ (filled symbols) for PSHQ9 in steady state at different temperatures in the nematic region: $(\circ, \bullet) 130^{\circ} \mathrm{C} ;(\Delta, \boldsymbol{\Delta}) 140^{\circ} \mathrm{C} ;(\square, \boldsymbol{\square}) 150^{\circ} \mathrm{C}$. The fitting curves for $\eta$ vs $\dot{\gamma}$ plots at different temperatures: (solid line) $130{ }^{\circ} \mathrm{C}$, (long dash) $140^{\circ} \mathrm{C}$, and (short dash) $150^{\circ} \mathrm{C}$, as well as for $\mathrm{N}_{1}$ vs $\dot{\gamma}$ plots at different temperatures: (dotted line) $130{ }^{\circ} \mathrm{C}$, (dash-dot) $140^{\circ} \mathrm{C}$, and (dash-dot-dot) $150^{\circ} \mathrm{C}$. The viscosities of both experimental data and fitting curves exhibit regions II and III, and the Newtonian behavior becomes obvious as the temperature increases. Both experimental and simulated data for $\mathrm{N}_{1}$ are positive over the range of shear rates investigated. The viscosity of PSHQ9 exhibits a shear-thinning at very low values of $\dot{\gamma}$, a Newtonian behavior at its intermediate values, and then, again, a strong shear-thinning behavior at higher values of $\dot{\gamma}$. However, as the temperature is increased from 130 to $140{ }^{\circ} \mathrm{C}$, region I in the logarithmic plot $\eta$ vs $\dot{\gamma}$ for PSHQ9 becomes very weak, and as the temperature is increased futher to $150{ }^{\circ} \mathrm{C}$, 


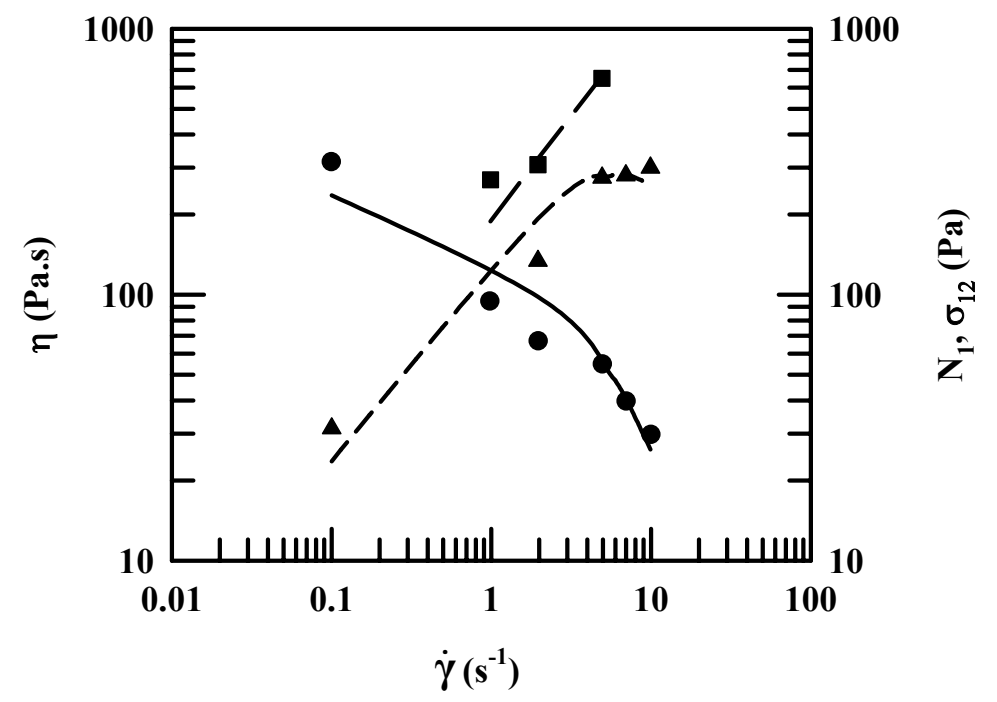

Figure 5.1 Logarithmic plots of experimental data of viscosity $(\eta, \bullet)$, shear stress $\left(\sigma_{12}, \boldsymbol{\Lambda}\right)$, and first normal stress difference $\left(\mathrm{N}_{1}, \boldsymbol{\square}\right)$ versus shear rate $(\dot{\gamma})$ for Titan at 340 ${ }^{\circ} \mathrm{C}$, with fitting curves of $\eta$ (solid line), $\sigma_{12}$ (short dash line), and $\mathrm{N}_{1}$ (long dash line).

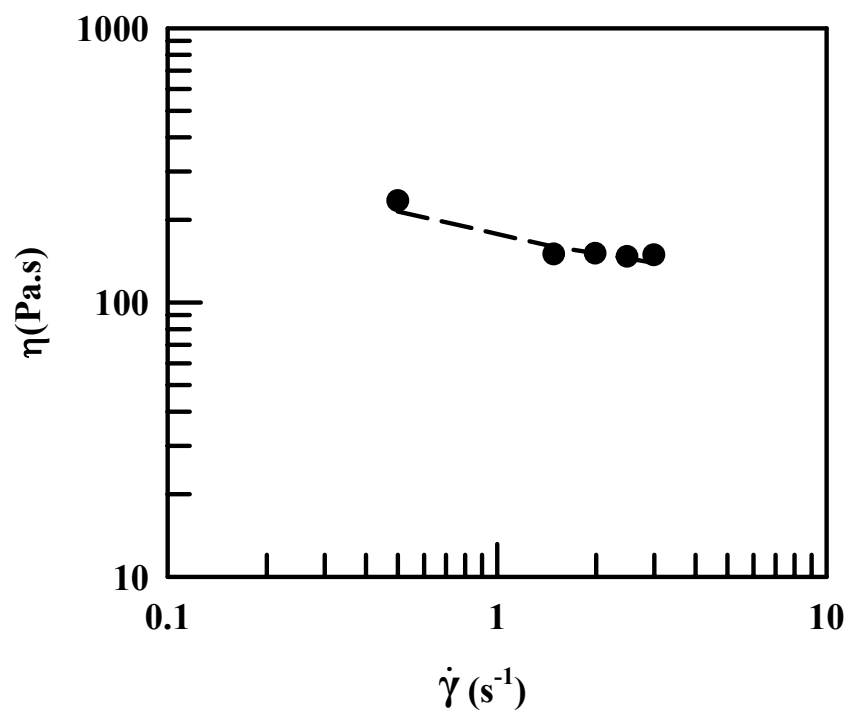

Figure 5.2 Plot of viscosity $(\eta)$ versus shear rate $(\dot{\gamma})$ for Zenite 6000 at $360{ }^{\circ} \mathrm{C}$ : experimental data $(\bullet)$ and fitting curve (dashed line). 


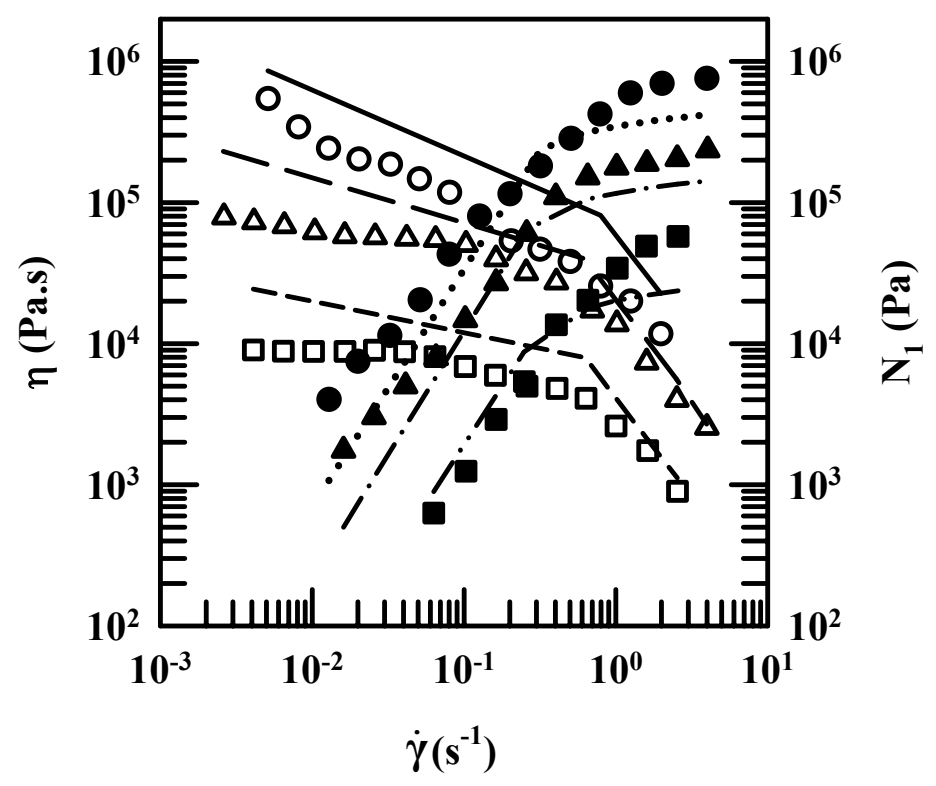

Figure 5.3 Plots of experimental data of $\log \eta$ vs $\log \dot{\gamma}$ (open symbols) and $\log \mathrm{N}_{1}$ vs $\log \dot{\gamma}$ (filled symbols) for PSHQ9 in steady flow at different temperatures in the nematic region: $(\circ, \bullet) 130^{\circ} \mathrm{C} ;(\Delta, \boldsymbol{\Delta}) 140^{\circ} \mathrm{C} ;(\square, \boldsymbol{\square}) 150^{\circ} \mathrm{C}$. The fitting curves for plots of $\log \eta$ vs $\log \dot{\gamma}$ at different temperatures: (solid line) $130{ }^{\circ} \mathrm{C}$, (long dash line) $140^{\circ} \mathrm{C}$, and (short dash line) $150^{\circ} \mathrm{C}$, as well as for $\log \mathrm{N}_{1}$ vs $\log \dot{\gamma}$ plots at different temperatures: (dotted line) $130^{\circ} \mathrm{C}$, (dash-dot line) $140^{\circ} \mathrm{C}$, and (dash-dot-dot line) $150^{\circ} \mathrm{C}$.

the region I disappears. Since PSHQ9 is a polydisperse polymer, the low molecular portion of PSHQ9 might begin to transform into the isotropic state, forming a biphasic, before reaching $\mathrm{T}_{\mathrm{NI}}\left(\mathrm{ca} .160{ }^{\circ} \mathrm{C}\right.$ ). Thus, the nematicity of PSHQ9 becomes progressively weaker as the temperature approaches its $\mathrm{T}_{\mathrm{NI}}$. In such circumstance, region $\mathrm{I}$ in the logarithmic plot $\eta$ vs $\dot{\gamma}$ for PSHQ9 may not be observable.

Figure 5.4 shows the logarithmic plots of experimental data for $\eta$ vs $\dot{\gamma}$ (open symbols) and $\mathrm{N}_{1}$ vs $\dot{\gamma}$ (filled symbols) for PI-14-5CN in steady shearing flows at different temperatures in the nematic region: $(\circ, \bullet) 70^{\circ} \mathrm{C} ;(\Delta, \boldsymbol{\Delta}) 80^{\circ} \mathrm{C} ;(\square, \boldsymbol{\square}) 90^{\circ} \mathrm{C}$. The fitting curves for the plots of $\eta$ vs $\dot{\gamma}$ at different temperatures: (solid line) $70^{\circ} \mathrm{C}$, (long dash) 


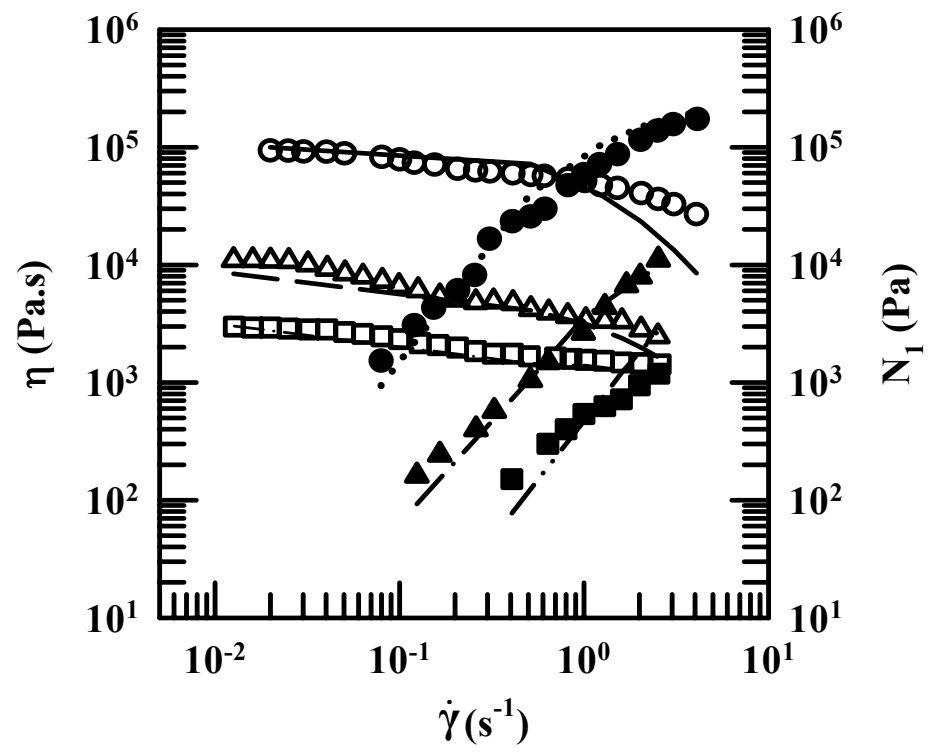

Figure 5.4 Plots of experimental data of $\log \eta$ vs $\log \dot{\gamma}$ (open symbols) and $\log \mathrm{N}_{1}$ vs $\log \dot{\gamma}$ (filled symbols) for PI-14-5CN in steady state at different temperatures in the nematic region: $(\circ, \bullet) 70^{\circ} \mathrm{C} ;(\Delta, \boldsymbol{\Delta}) 80^{\circ} \mathrm{C} ;(\square, \boldsymbol{\square}) 90^{\circ} \mathrm{C}$. The fitting curves for $\log \eta$ vs $\log \dot{\gamma}$ plots at different temperatures: (solid line) $70^{\circ} \mathrm{C}$, (long dash line) $80^{\circ} \mathrm{C}$, and (short dash line) $90^{\circ} \mathrm{C}$, as well as for $\log \mathrm{N}_{1}$ vs $\log \dot{\gamma}$ plots at different temperatures: (dotted line) $70^{\circ} \mathrm{C}$, (dash-dot line) $80^{\circ} \mathrm{C}$, and (dash-dot-dot line) $90^{\circ} \mathrm{C}$.

$80^{\circ} \mathrm{C}$, and (short dash) $90^{\circ} \mathrm{C}$, as well as for $\mathrm{N}_{1}$ vs $\dot{\gamma}$ plots at different temperatures: (dotted line) $70^{\circ} \mathrm{C}$, (dash-dot) $80^{\circ} \mathrm{C}$, and (dash-dot-dot) $90^{\circ} \mathrm{C}$. It can be seen that the fitting curves for both $\eta$ and $\mathrm{N}_{1}$ are in an excellent agreement with experimental data, being better than those for PSHQ9. The viscosity of PI-14-5CN exhibits a Newtonian behavior at low $\dot{\gamma}$ and then shear-thinning behavior, while the $\mathrm{N}_{1}$ of PI-14-5CN is positive over the entire range of $\dot{\gamma}$ tested at three temperatures in the nematic region. The absence of region I in the $\log \eta$ vs $\log \dot{\gamma}$ plots might be due to the fact that PI-14-5CN is a side-chain LCP, and its nematicity might not be sufficiently strong. The above observation supports the view that region I in the plots of $\eta$ vs $\dot{\gamma}$ for an LCP is associated with the existence of a domain structure [7]. 


\subsection{Simulated Results for Transient Start-up Shear Flow}

The appearance of multiple overshoots in the first normal stress difference in transient shear flow can be predicted by Marrucci-Maffettone theory [67]. However, Marrucci-Maffettone theory for monodomain is based on the Maier-Saupe potential, which may be valid for small-molecular thermotropic liquid crystals. Therefore, this theory is not suitable for semiflexible main-chain LCP exhibiting flow aligning behavior. On the other hand, the Larson-Doi mesoscopic model [81] considers the evolution of texture based on some experimental observations describing the decrease in domain size with increasing shear rate and growing upon cessation of flow. Although the Larson-Doi mesoscopic model predicts qualitatively the experimental observations for the time evolution of $\sigma^{+}(\dot{\gamma}, \mathrm{t}) / \sigma$ and $\mathrm{N}_{1}^{+}(\dot{\gamma}, \mathrm{t}) / \mathrm{N}_{1}$ for LCP's in transient shear flow, the predicted magnitude of $\sigma^{+}(\dot{\gamma}, \mathrm{t})$ is lower than that of $\mathrm{N}_{1}{ }^{+}(\dot{\gamma}, \mathrm{t}) / \mathrm{N}_{1}$, which is contrary to experimental observations [109]. Further, the models predict a much shorter transient time (strain) of $\sigma^{+}(\dot{\gamma}, \mathrm{t}) / \sigma$ and $\mathrm{N}_{1}^{+}(\dot{\gamma}, \mathrm{t}) / \mathrm{N}_{1}$ variations compared with experimental results. The inadequacy of the Larson-Doi model to accurately predict the time evolution of $\mathrm{N}_{1}^{+}(\dot{\gamma}, \mathrm{t})$ in transient shear flow of LCP may be attributable to the presence of long flexible spacers and bulky pendent side groups in the LCP. Both the long flexible spacers and bulky pendent side groups present in the LCP might directly suppress molecular rotations and thus, perphase, collective molecular rotations, or director tumbling. Although the LarsonDoi mesoscopic model is virtually the only model available in the literature that incorporates polydomain texture and distortional elastic effects, the model is based on the Leslie-Ericksen equations, which may be more appropriate for small molecule liquid 
crystals or lyotropic systems that have short relaxation times. To the best of our knowledge, most of the thermotropic LCP's exhibit flow aligning behavior. Therefore, the Leonov's viscoelastic nematodynamics can be used to elucidate the experimental observations for these polymers.

Figures 5.5 and 5.6 describe time evolution of normalized shear stress $\sigma^{+}(\dot{\gamma}, \mathrm{t}) / \sigma$ and first normal stress difference $\mathrm{N}_{1}^{+}(\dot{\gamma}, \mathrm{t}) / \mathrm{N}_{1}$ with strain $(\dot{\gamma} \mathrm{t})$ for Titan at 340 ${ }^{\circ} \mathrm{C}$ in the start-up shear flow at $\dot{\gamma}=6 \mathrm{~s}^{-1}$, respectively. Here experimental data are denoted by dots $(\bullet)$ and fitting curve by dashed line. It can be seen that both $\sigma^{+}(\dot{\gamma}, \mathrm{t}) / \sigma$ and $\mathrm{N}_{1}^{+}(\dot{\gamma}, \mathrm{t}) / \mathrm{N}_{1}$ have a large and broad overshoot at $\dot{\gamma}$ t of about 50 . Since Titan has a very rigid molecule, the evolution of texture is slow, and in turn the overshoot occurs at high value of $\dot{\gamma}$.

Figure 5.7 shows the time evolution of shear stress $\sigma$ with strain $(\dot{\gamma} \mathrm{t})$ for Zenite 6000 at $360{ }^{\circ} \mathrm{C}$ in start-up shear flow at $\dot{\gamma}=2 \mathrm{~s}^{-1}$, where experimental data shown by dots $(\bullet)$ and fitting curve by dashed line. The experimental data for shear stress exhibit first a small overshoot at $\dot{\gamma}$ t of ca. 4 and a large and broad overshoot at $\dot{\gamma}$ t of ca. 76 . For the fitting curve, a small overshoot occurs at $\dot{\gamma}$ t of ca. 16 , and a large overshoot at $\dot{\gamma}$ t of ca. 50.

Figures 5.8 and 5.9 describe the time evolution of normalized shear stress $\sigma^{+}(\dot{\gamma}, \mathrm{t}) / \sigma$ and first normal stress difference $\mathrm{N}_{1}^{+}(\dot{\gamma}, \mathrm{t}) / \mathrm{N}_{1}$ with strain $\dot{\gamma}$ t for PSHQ9 at 130 ${ }^{\circ} \mathrm{C}$ in start-up shear flow at $\dot{\gamma}=1 \mathrm{~s}^{-1}$, respectively, in which experimental data are shown by dots $(\bullet)$ and fitting curve by dashed line. The simulated values of overshoots for $\sigma^{+}(\dot{\gamma}, \mathrm{t}) / \sigma$ and $\mathrm{N}_{1}^{+}(\dot{\gamma}, \mathrm{t}) / \mathrm{N}_{1}$ are the same as those of experimental data, but the overshoots 


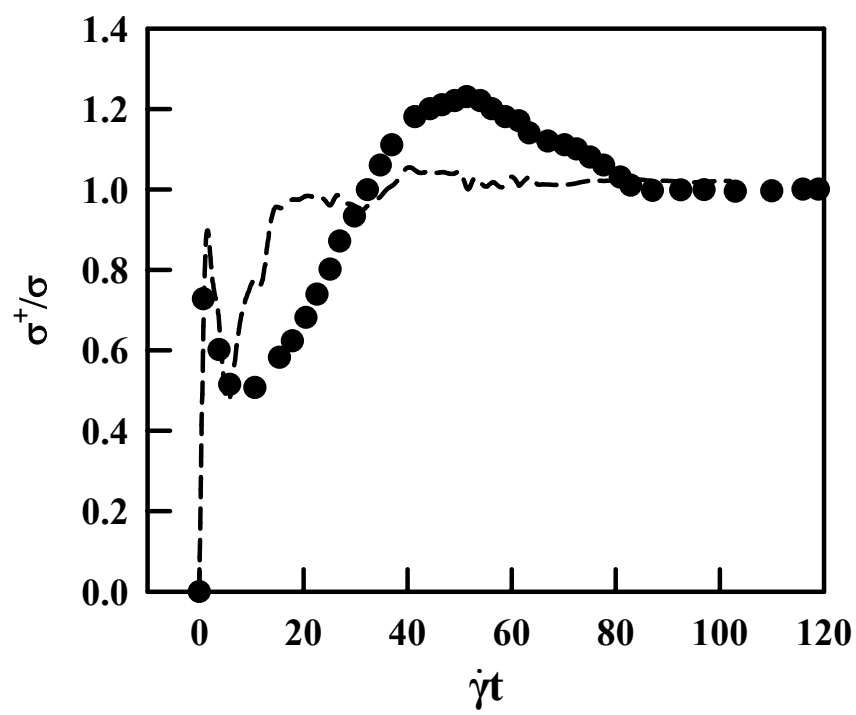

Figure 5.5 Time evolution of normalized shear stress $\sigma^{+}(\dot{\gamma}, \mathrm{t}) / \sigma$ with strain $(\dot{\gamma} \mathrm{t})$ for Titan at $340{ }^{\circ} \mathrm{C}$ in start-up shear flow at $\dot{\gamma}=6 \mathrm{~s}^{-1}$ : experimental data $(\bullet)$ and fitting curve (dashed line).

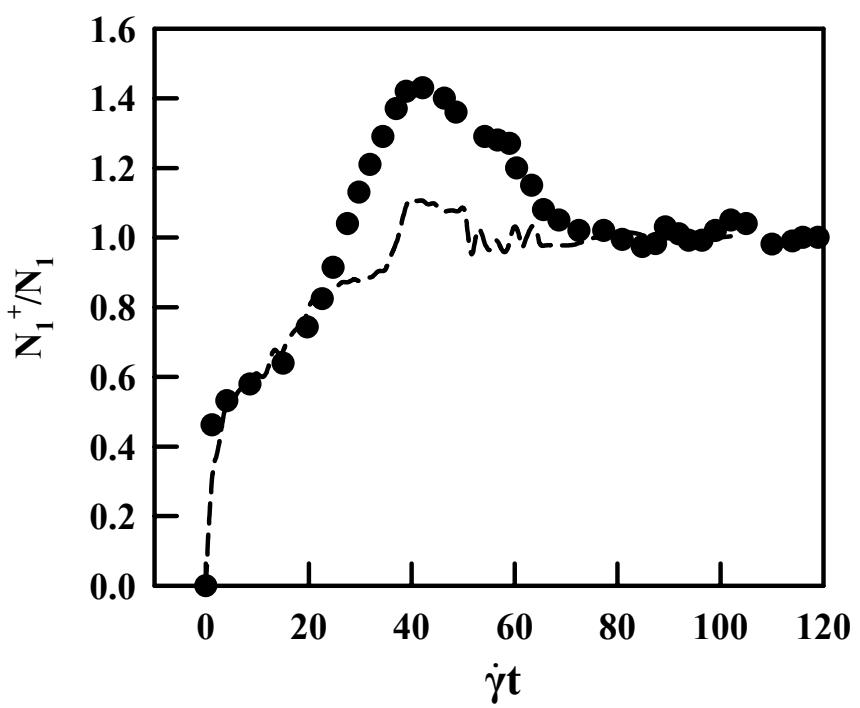

Figure 5.6 Time evolution of normalized first normal stress difference $\mathrm{N}_{1}{ }^{+}(\dot{\gamma}, \mathrm{t}) / \mathrm{N}_{1}$ with strain $(\dot{\gamma} \mathrm{t})$ for Titan at $340{ }^{\circ} \mathrm{C}$ in start-up shear flow at $\dot{\gamma}=6 \mathrm{~s}^{-1}$ : experimental data $(\bullet)$ and fitting curve (dashed line). 


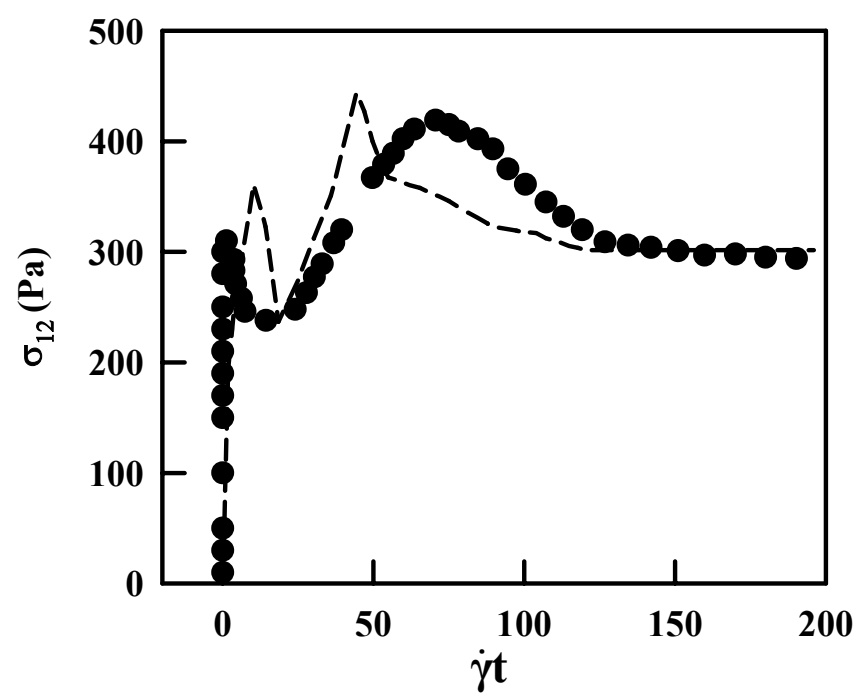

Figure 5.7 Time evolution of shear stress $(\sigma)$ with strain $(\dot{\gamma} \mathrm{t})$ for Zenite 6000 at $360{ }^{\circ} \mathrm{C}$ in start-up shear flow at $\dot{\gamma}=2 \mathrm{~s}^{-1}$ : experimental data $(\bullet)$ and fitting curve (dashed line).

of fitting curves occur at a relatively low $\dot{\gamma}$ t and are very narrow. Such large values of $\sigma^{+}(\dot{\gamma}, \mathrm{t}) / \sigma$ ratio is believed to be characteristic of liquid crystalline polymers, as a consequence of the existence of a lot of polydomains in the nematic state in start-up flow. It should be pointed out that the theory used for simulation utilizes monodomain approach, whereas PSHQ9 exhibits polydomains in nematic state in start-up flow. As a consequence, the appreciable deviation of simulated results from experimental results seems reasonable to occur.

Figures 5.10 and 5.11 describe the time evolution of normalized shear stress $\sigma^{+}(\dot{\gamma}, \mathrm{t}) / \sigma$ and first normal stress difference $\mathrm{N}_{1}{ }^{+}(\dot{\gamma}, \mathrm{t}) / \mathrm{N}_{1}$ with strain $(\dot{\gamma} \mathrm{t})$ for PI-14-5CN at $70{ }^{\circ} \mathrm{C}$ upon start-up of shear flow at $\dot{\gamma}=1 \mathrm{~s}^{-1}$, respectively, in which experimental data are shown by dots $(\bullet)$ and fitting curve by dashed line. Both $\sigma^{+}(\dot{\gamma}, \mathrm{t}) / \sigma$ and $\mathrm{N}_{1}{ }^{+}(\dot{\gamma}, \mathrm{t}) / \mathrm{N}_{1}$ of experimental data exhibit large overshoots and then decay to steady values. The 


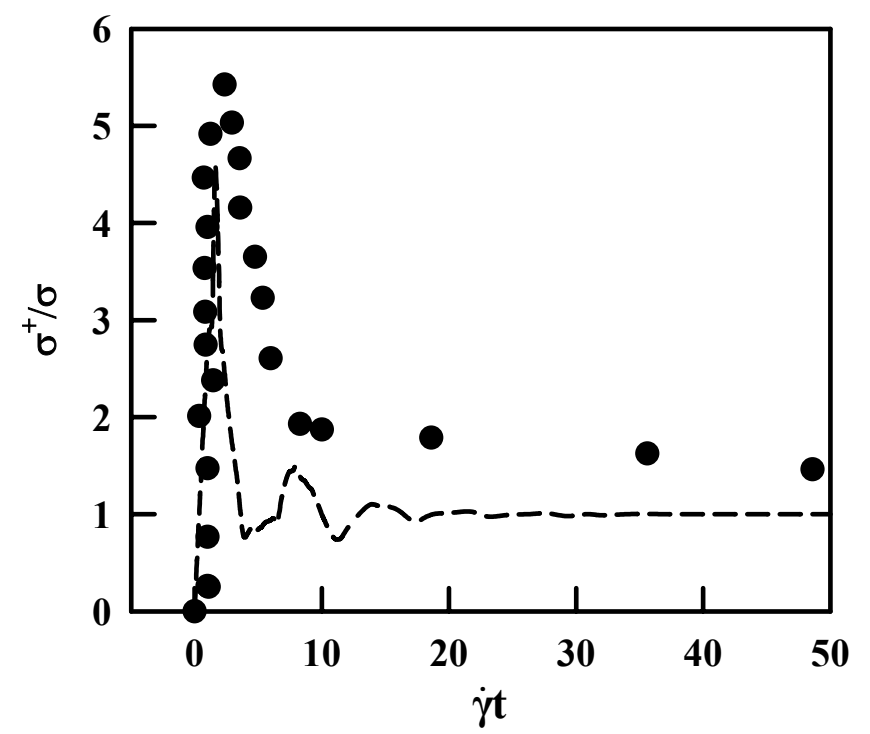

Figure 5.8 Time evolution of normalized shear stress $\sigma^{+}(\dot{\gamma}, \mathrm{t}) / \sigma$ with strain $(\dot{\gamma} \mathrm{t})$ for PSHQ9 at $130{ }^{\circ} \mathrm{C}$ in start-up shear flow at $\dot{\gamma}=1 \mathrm{~s}^{-1}$ : experimental data $(\bullet)$ and fitting curve (dashed line).

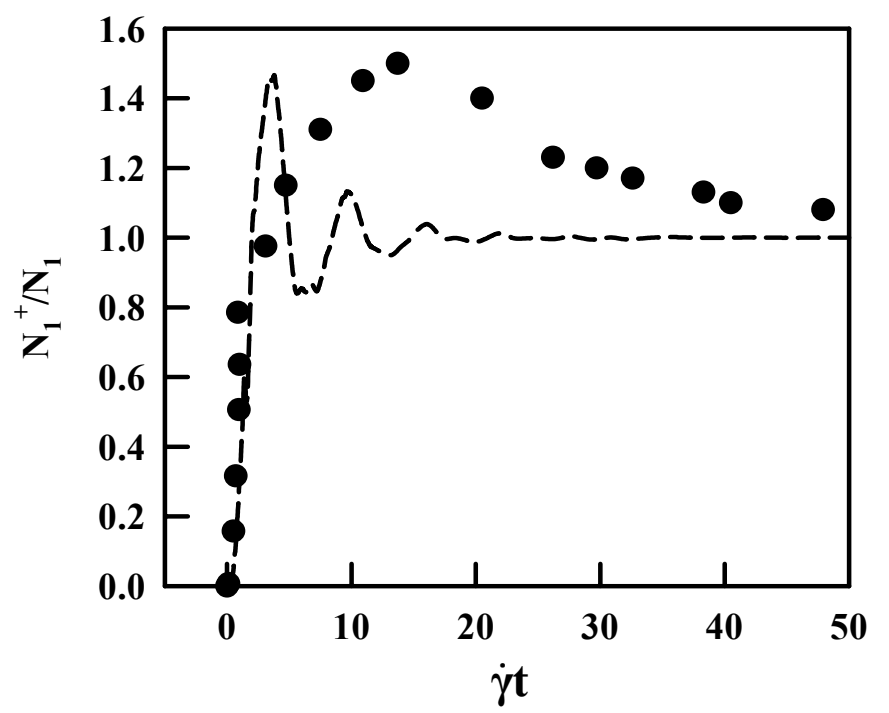

Figure 5.9 Time evolution of normalized first normal stress difference $\mathrm{N}_{1}{ }^{+}(\dot{\gamma}, \mathrm{t}) / \mathrm{N}_{1}$ with

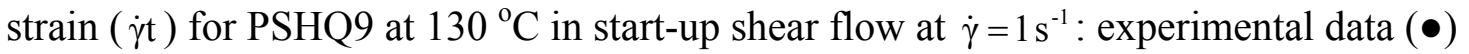
and fitting curve (dashed line). 
simulated time evolution of normalized shear stress with strain is fitted well enough with experimental data, whereas there is a relatively large difference between the fitting curve of $\mathrm{N}_{1}^{+}(\dot{\gamma}, \mathrm{t}) / \mathrm{N}_{1}$ and experimental data, which may be attributable to the fact that we employed the Leonov's viscoelastic nematodymanics based on the theory of monodomain to simulate the polydomain experimental data. As compared with Figures 5.8 and 5.9, in start-up shear flow the overshoot peak in $\mathrm{N}_{1}{ }^{+}(\dot{\gamma}, \mathrm{t}) / \mathrm{N}_{1}$ occurs much sooner in PI-14-5CN than in PSHQ9, while the overshoot peak in $\sigma^{+}(\dot{\gamma}, \mathrm{t}) / \sigma$ occurs very quickly in both PI-14-5CN and PSHQ9. The overshoot peak value of fitted $\mathrm{N}_{1}^{+}(\dot{\gamma}, \mathrm{t}) / \mathrm{N}_{1}$ of PSHQ9 is slightly larger than that of PI-14-5CN, while the overshoot peak value of $\sigma^{+}(\dot{\gamma}, \mathrm{t}) / \sigma$ of PSHQ9 is about 3-4 times greater than that of PI-14-5CN. Thus, the transient response in first normal stress difference and shear stress for side-chain LCP, PI-14-5CN, are quite different from those for main-chain LCP, PSHQ9.

Because the $5 \mathrm{CN}-\mathrm{COOH}$ mesogens are grafted onto the coillike PI forming a polymer backbone through five methylene groups as flexible spacer, the motions of the polymer backbone and the $5 \mathrm{CN}-\mathrm{COOH}$ mesogens in $\mathrm{PI}-14-5 \mathrm{CN}$ may be regarded as being partially decoupled, making the $5 \mathrm{CN}-\mathrm{COOH}$ mesogens mobile during shear flow [59]. Further, each mesogen grafted onto the backbone of PI-14-5CN may move or orient, upon start-up of shear flow, little dependent upon other mesogens. On the other hand, the mesongens in PSHQ9 are directly linked to the polymer backbone, making motions of the mesogens and backbone of PSHQ9 strongly coupled during shear flow. Thus, each mesogen in PSHQ9 can not act as if it were independent from other mesogens. All the mesogens in PSHQ9 must act, upon start-up of shear flow collectively or cooperatively. 


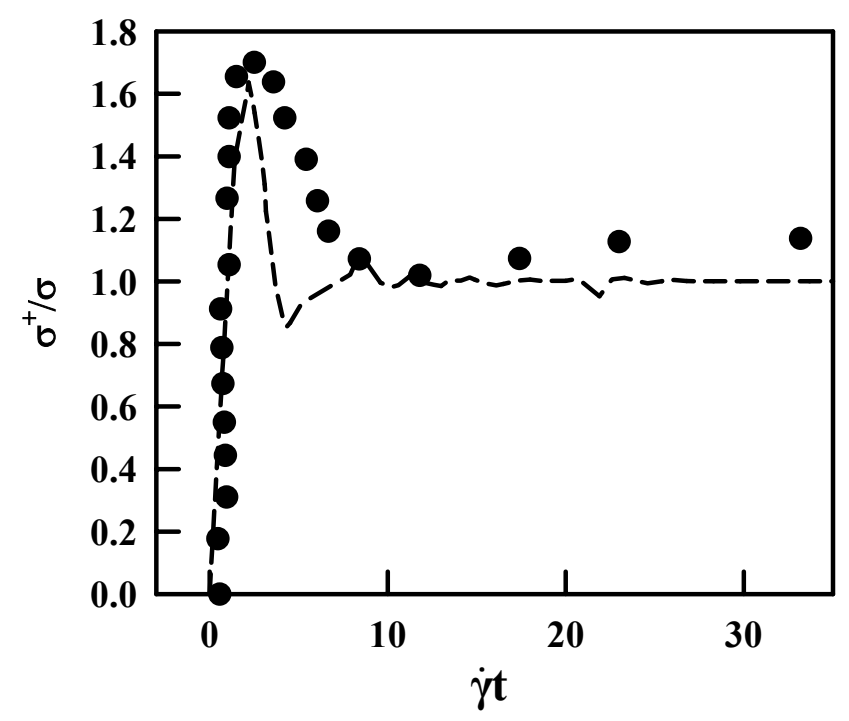

Figure 5.10 Time evolution of normalized shear stress $\sigma^{+}(\dot{\gamma}, \mathrm{t}) / \sigma$ with strain $(\dot{\gamma} \mathrm{t})$ for PI$14-5 \mathrm{CN}$ at $70{ }^{\circ} \mathrm{C}$ in start-up shear flow at $\dot{\gamma}=1 \mathrm{~s}^{-1}$ : experimental data (•) and fitting curve (dashed line).

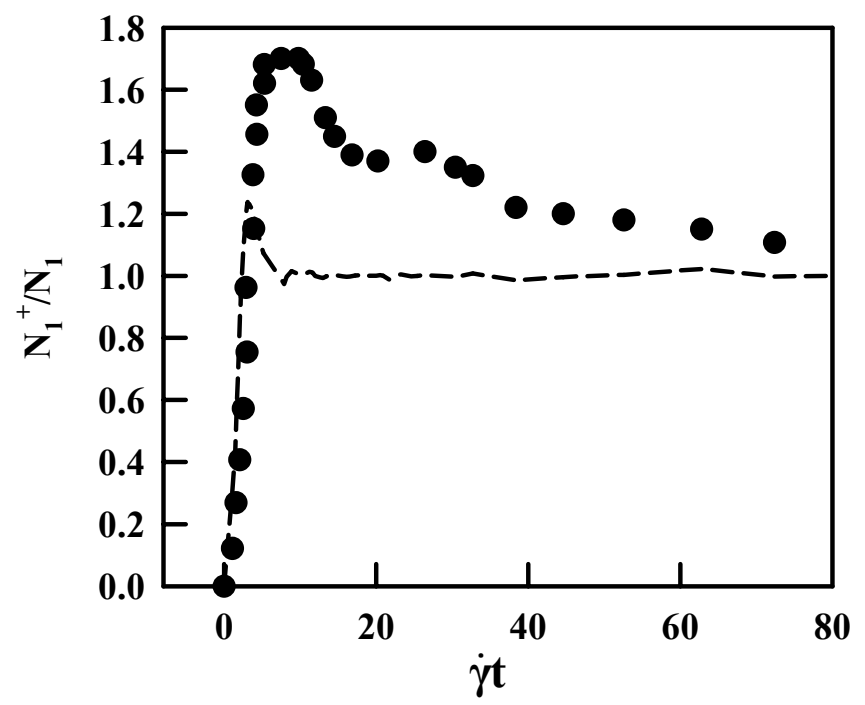

Figure 5.11 Time evolution of normalized first normal stress difference $\mathrm{N}_{1}{ }^{+}(\dot{\gamma}, \mathrm{t}) / \mathrm{N}_{1}$ with strain $(\dot{\gamma} \mathrm{t})$ for PI-14-5CN at $70{ }^{\circ} \mathrm{C}$ in start-up shear flow at $\dot{\gamma}=1 \mathrm{~s}^{-1}$ : experimental data (•) and fitting curve (dashed line). 
5.6 Simulated Results for Relaxation upon Cessation of Steady Flow

Figures 5.12 and 5.13 describe the relaxation of shear stress $\left(\sigma_{12}\right)$ and first normal stress difference $\left(\mathrm{N}_{1}\right)$ for Titan at $340{ }^{\circ} \mathrm{C}$ upon cessation of steady flow with shear rate $\dot{\gamma}=6 \mathrm{~s}^{-1}$, respectively. Here experimental data are shown by dots $(\bullet)$ and fitting curve by dashed lines. The values of both $\sigma_{12}$ and $\mathrm{N}_{1}$ upon relaxation drop abruptly and reach zero at the time of around $4 \mathrm{~s}$. The fitting curve of $\sigma_{12}$ has an excellent agreement with the experimental data, but this is not the case for $\mathrm{N}_{1}$ when $\mathrm{t}>4 \mathrm{~s}$. This may be attributable to the experimental error, because the final value of $\mathrm{N}_{1}$ during relaxation should reach zero.

Figure 5.14 presents the relaxation of normalized (a) shear stress, $\sigma^{-}(\dot{\gamma}, t) / \sigma_{0}$ and (b) first normal stress difference, $N_{1}^{-}(\dot{\gamma}, t) / N_{1,0}$ upon cessation of steady shear flow at $\dot{\gamma}$ $=0.5 \mathrm{~s}^{-1}$ for PSHQ9 at $130^{\circ} \mathrm{C}:(\bullet)$ experimental data and (dashed line) fitting curve. Here $\sigma_{0}$ and $N_{1,0}$ are the steady-state shear stress and first normal stress difference just prior to flow cessation, respectively. It is seen that $\sigma^{-}(\dot{\gamma}, t) / \sigma_{0}$ approaches to zero at $t_{R}>7 \mathrm{~s}$, whereas $N_{1}^{-}(\dot{\gamma}, t) / N_{1,0}$ reaches zero when $t_{R}>15 \mathrm{~s}$. As shear rate $\dot{\gamma}$ increases from 0.5 $\mathrm{s}^{-1}$ to $1.0 \mathrm{~s}^{-1}$, as indicated in Figure 5.15, the relaxation rate of shear stress and first normal stress difference is slower as the applied shear rate during steady shearing is increased. This is attributable to the fact that the higher the applied shear rate, the greater the respective value of shear stresses and thus the longer it will take for the shear stress to completely relax after cessation of shear flow.

Figure 5.16 presents the relaxation of normalized shear stress, $\sigma^{-}(\dot{\gamma}, t) / \sigma_{0}$ and first normal stress difference, $N_{1}^{-}(\dot{\gamma}, t) / N_{1,0}$ upon cessation of steady shear flow at $\dot{\gamma}=0.5 \mathrm{~s}^{-1}$ 


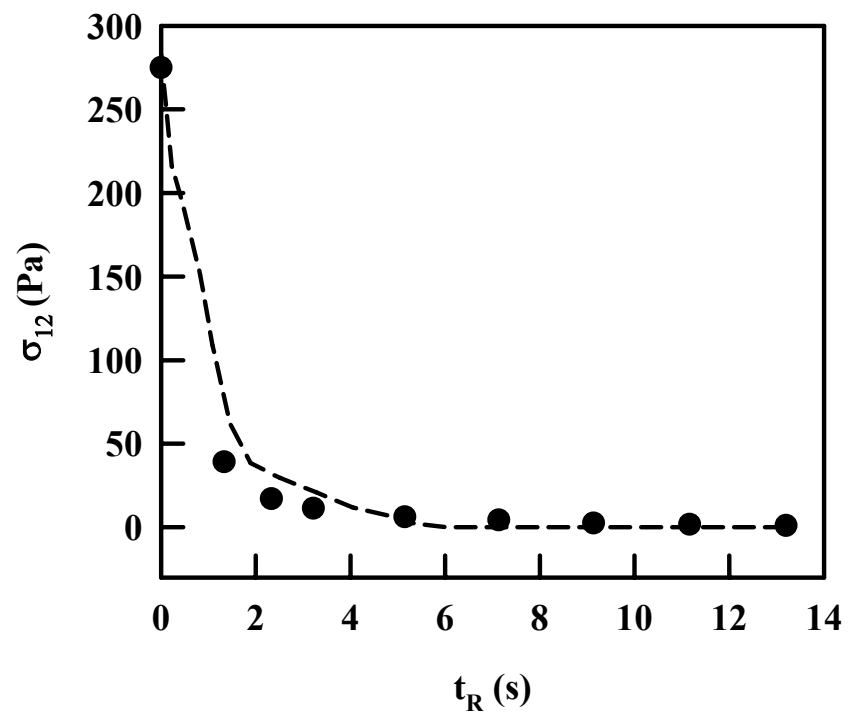

Figure 5.12 Relaxation of shear stress $\left(\sigma_{12}\right)$ for Titan at $340{ }^{\circ} \mathrm{C}$ upon cessation of steady flow at shear rate $\dot{\gamma}=6 \mathrm{~s}^{-1}$ : experimental data $(\bullet)$ and fitting curve (dashed line).

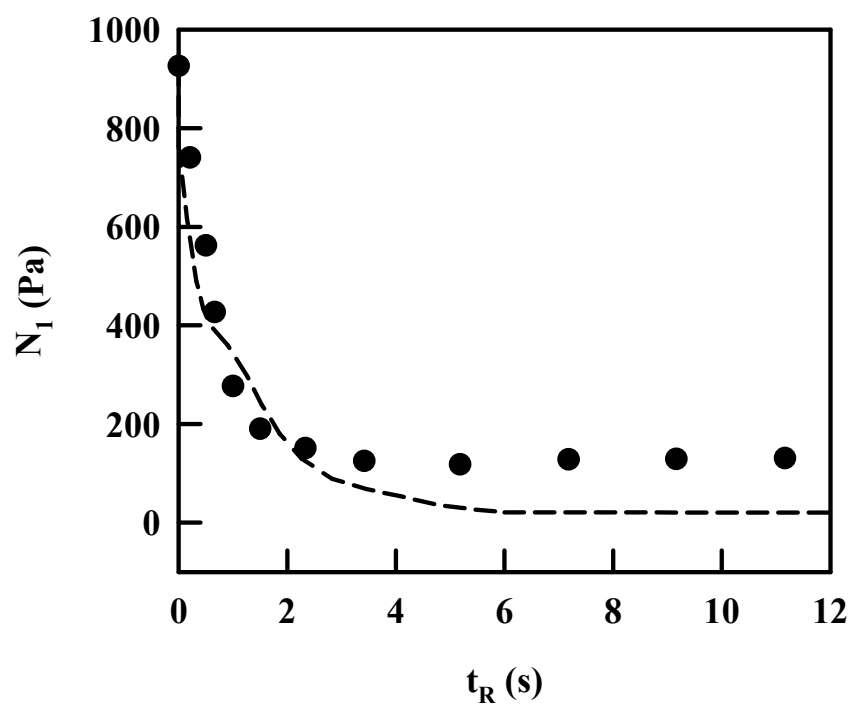

Figure 5.13 Relaxation of first normal stress difference $\left(\mathrm{N}_{1}\right)$ for Titan at $340{ }^{\circ} \mathrm{C}$ upon cessation of steady flow at shear rate $\dot{\gamma}=6 \mathrm{~s}^{-1}$ : experimental data $(\bullet)$ and fitting curve (dashed line). 

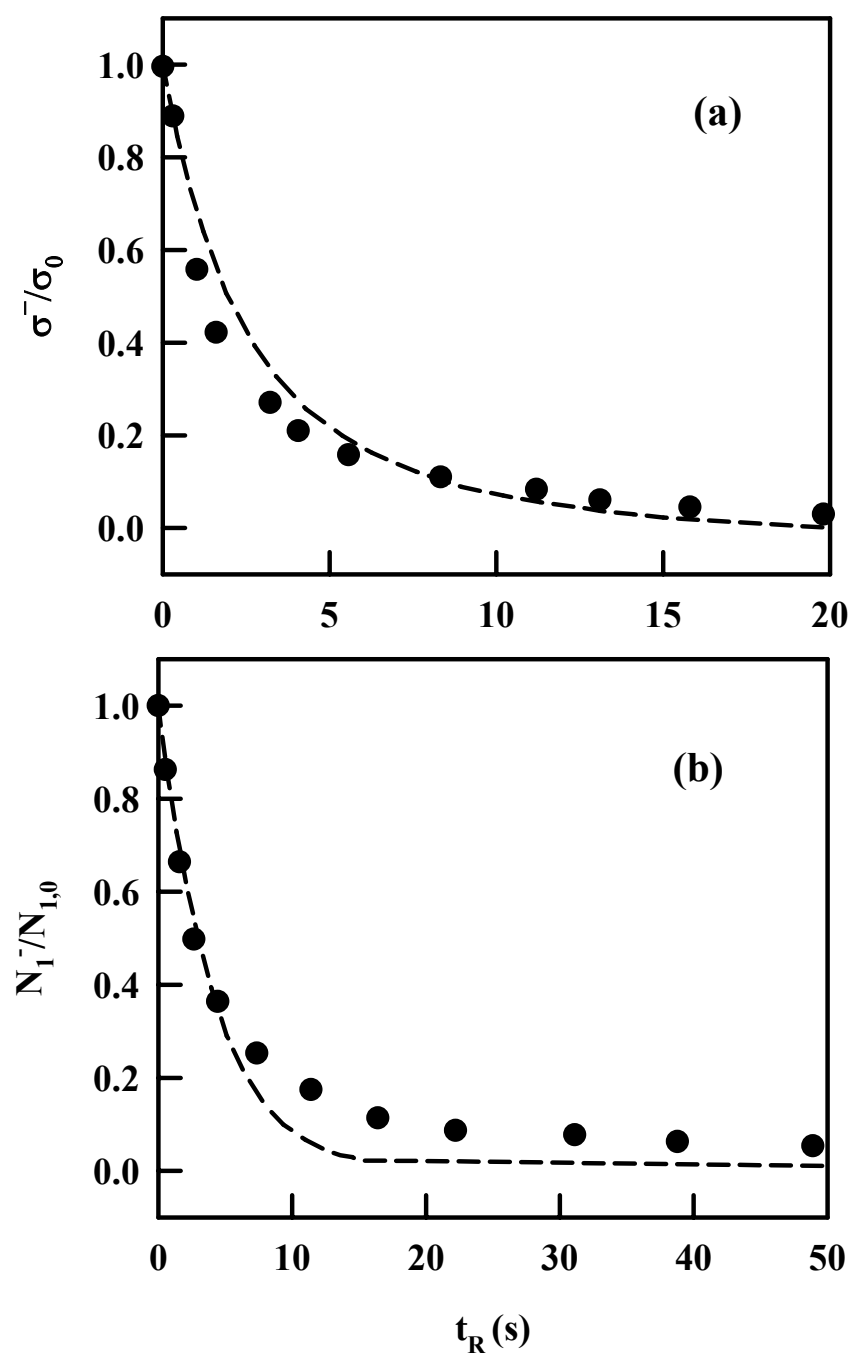

Figure 5.14 Relaxation of normalized (a) shear stress, $\sigma^{-}(\dot{\gamma}, t) / \sigma_{0}$ and (b) first normal stress difference, $N_{1}^{-}(\dot{\gamma}, t) / N_{1,0}$ upon cessation of steady shear flow at $\dot{\gamma}=0.5 \mathrm{~s}^{-1}$ for PSHQ9 at $130^{\circ} \mathrm{C}:(\bullet)$ experimental data and (dashed line) fitting curve. Here $\sigma_{0}$ and $N_{1,0}$ are the steady shear stress and first normal stress difference just prior to flow cessation, respectively. 

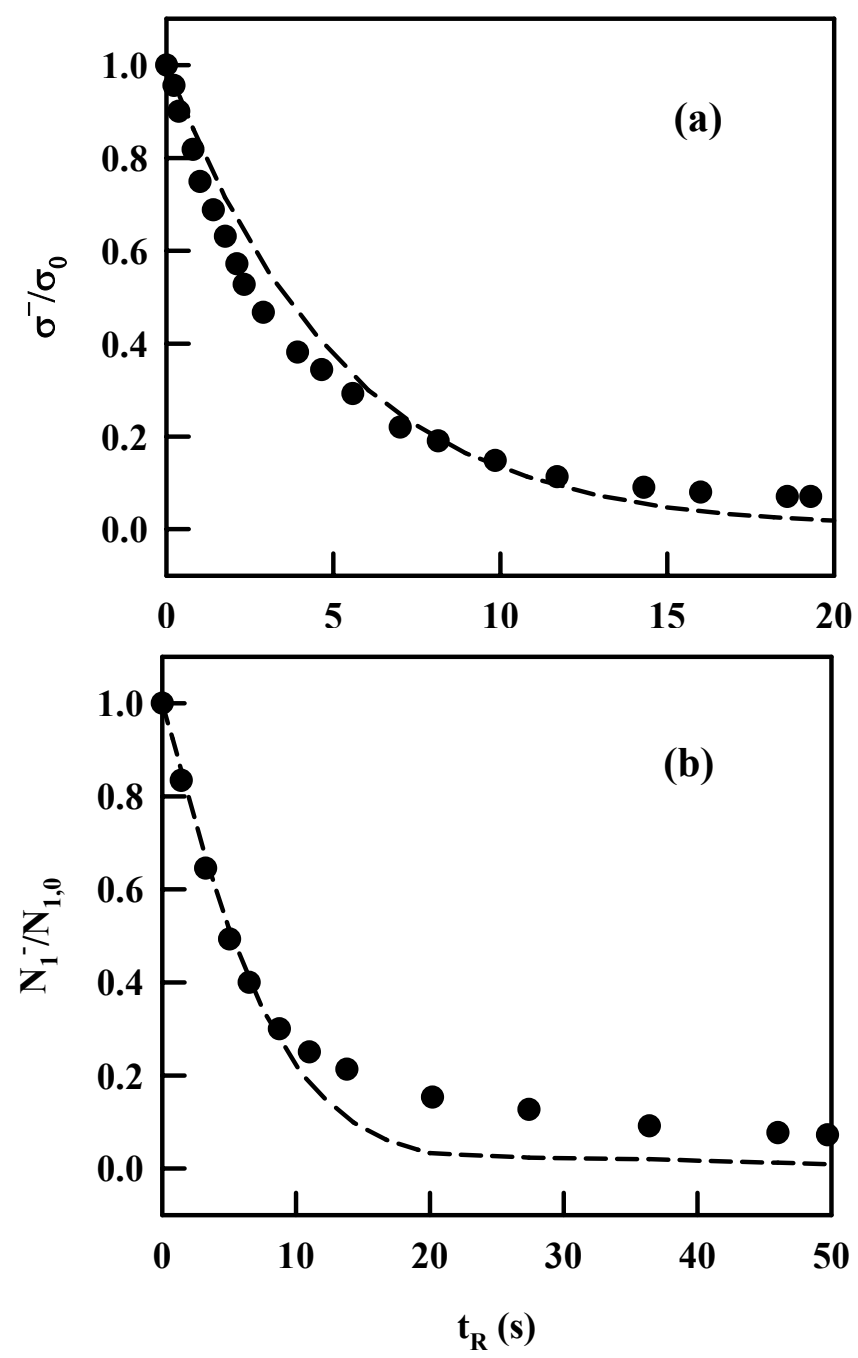

Figure 5.15 Relaxation of normalized (a) shear stress, $\sigma^{-}(\dot{\gamma}, t) / \sigma_{0}$ and (b) first normal stress difference, $N_{1}^{-}(\dot{\gamma}, t) / N_{1,0}$ upon cessation of steady shear flow at $\dot{\gamma}=1 \mathrm{~s}^{-1}$ for PSHQ9 at $130^{\circ} \mathrm{C}:(\bullet)$ experimental data and (dashed line) fitting curve. Here $\sigma_{0}$ and $N_{1,0}$ are the steady shear stress and first normal stress difference just prior to flow cessation, respectively. 
for PI-14-5CN at $70^{\circ} \mathrm{C}:(\bullet)$ experimental data and dashed lines show the fitting curves. Here $\sigma_{0}$ and $N_{1,0}$ are the steady-state shear stress and first normal stress difference just prior to flow cessation, respectively. As compared with Figure 5.14, the relaxation rate of shear stress and first normal stress difference in PI-14-5CN is much faster than in PSHQ9. This difference indicates how fast the recovery of the domain texture in sidechain LCP, PI-14-5CN is, after cessation of shear flow, as compared to that in main-chain LCP, PSHQ9. The 5CN-COOH grafted on the coillike backbone of PI, forming PI-14$5 \mathrm{CN}$, might be very mobile and thus would relax rather quickly upon cessation of shear flow, as compared to the mesogens that are linked directly to the polymer backbone of PSHQ9. As expected, it can be seen from Figure 5.17 that the relaxation of $\sigma^{-}(\dot{\gamma}, t) / \sigma_{0}$ and $N_{1}^{-}(\dot{\gamma}, t) / N_{1,0}$ of PI-14-5CN becomes relatively slower as $\dot{\gamma}$ increases from 0.5 to $1.0 \mathrm{~s}^{-1}$.

5.7 On the Time-Temperature Superposition for Weakly Viscoelastic Nematodynamics During the simulation of PSHQ9 and PI-14-5CN, the constitutive parameters $r_{1}, r_{2}$, $\lambda_{e}, \lambda_{v}, \hat{a}_{1}$, and $\hat{\beta}$ characterizing the anisotropy of nematics, were supposed to be temperature independent. Thus the only two parameters, characteristic relaxation time $\theta_{0}$ and viscosity $\eta_{0}$ were assumed changing with temperature. In this case the general equations of weak viscoelastic nematodynamics and their simple shearing specification (4-30) and (4-32) allow for the following scaling transformation:

$$
\sigma_{i j} \rightarrow \hat{\sigma}_{i j}=\sigma_{i j} \theta_{0} / \eta_{0}, t \rightarrow \hat{t}=t / \theta_{0}, \dot{\gamma} \rightarrow \hat{\dot{\gamma}}=\dot{\gamma} \theta_{0}
$$



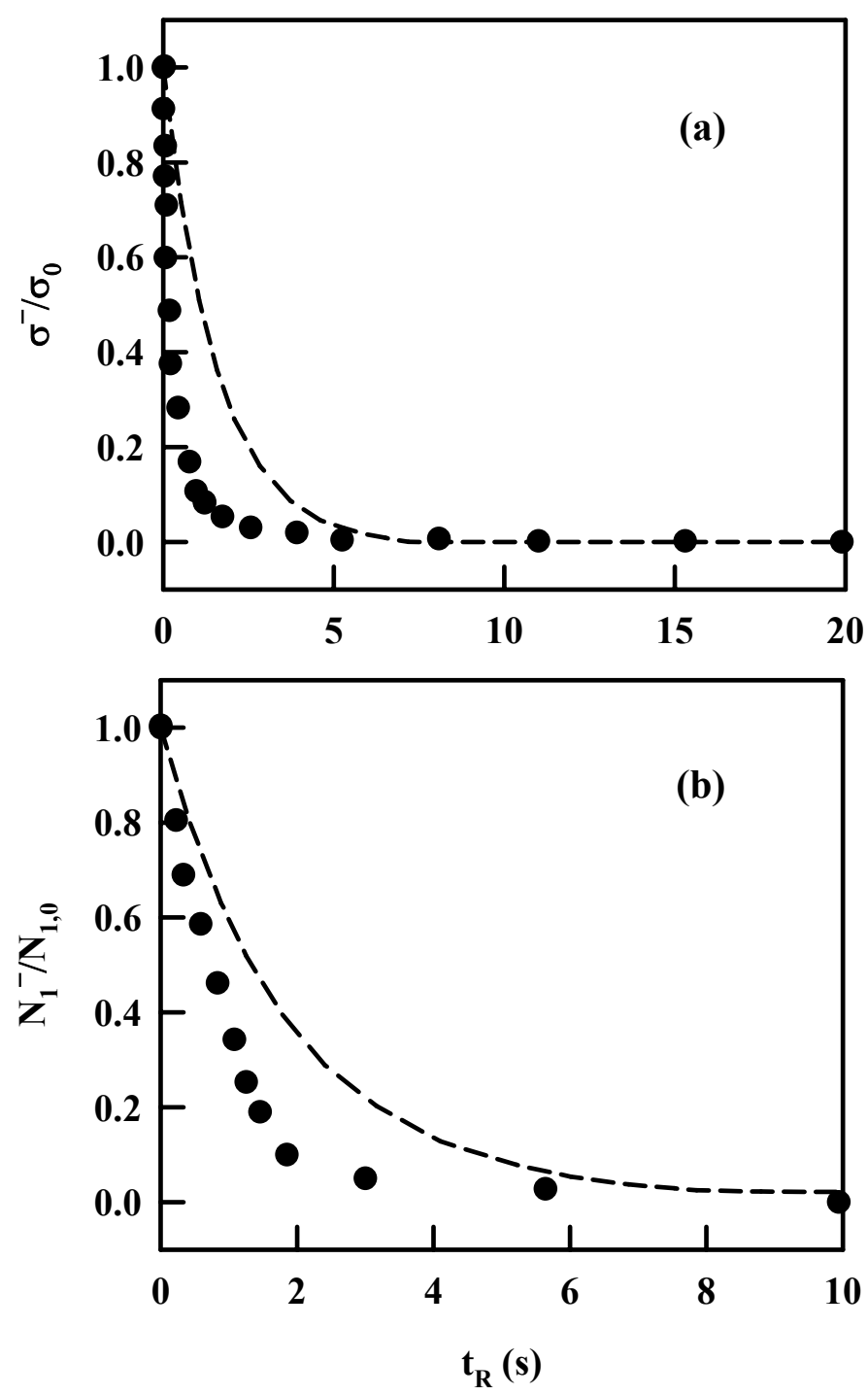

Figure 5.16 Relaxation of normalized (a) shear stress, $\sigma^{-}(\dot{\gamma}, t) / \sigma_{0}$ and (b) first normal stress difference, $N_{1}^{-}(\dot{\gamma}, t) / N_{1,0}$ upon cessation of steady shear flow at $\dot{\gamma}=0.5 \mathrm{~s}^{-1}$ for PI$14-5 \mathrm{CN}$ at $70^{\circ} \mathrm{C}:(\bullet)$ experimental data and (dashed line) fitting curve. Here $\sigma_{0}$ and $N_{1,0}$ are the steady-state shear stress and first normal stress difference just prior to flow cessation, respectively. 

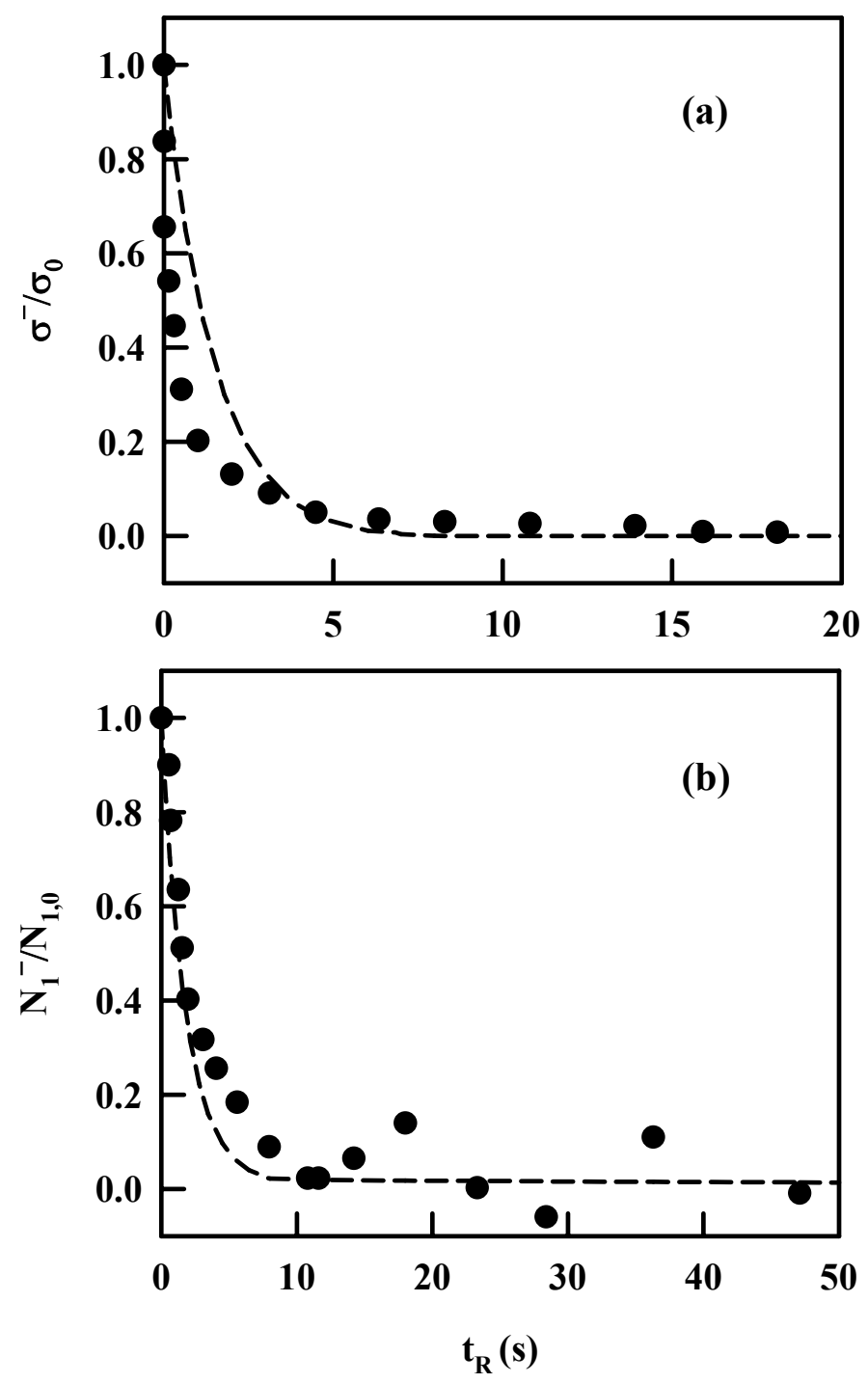

Figure 5.17 Relaxation of normalized (a) shear stress, $\sigma^{-}(\dot{\gamma}, t) / \sigma_{0}$ and (b) first normal stress difference, $N_{1}^{-}(\dot{\gamma}, t) / N_{1,0}$ upon cessation of steady shear flow at $\dot{\gamma}=1 \mathrm{~s}^{-1}$ for PI$14-5 \mathrm{CN}$ at $70^{\circ} \mathrm{C}:(\bullet)$ experimental data and (dashed line) fitting curve. Here $\sigma_{0}$ and $N_{1,0}$ are the steady-state shear stress and first normal stress difference just prior to flow cessation, respectively. 
The transformation (5-5) demonstrates the time-temperature superposition scaling. When non-dimensional variables, denoted in (5-5) by overcups, are introduced in equations (4-30) and (4-32), these equations will describe isothermal, generally nonsteady shearing for various constant temperatures as temperature independent curves. This is the time-temperature superposition principle for weak viscoelastic nematodynamics.

Since there was a lack of experimental time dependent data for the above LCP's, we will test below this time-temperature superposition principle for the available steady shearing curves. Here one can obtain some normalized non-dimensional dependencies of non-Newtonian viscosity $\hat{\eta}=\eta / \eta_{0}$ and the first normal stress difference $\hat{N}_{1}=N_{1} \theta_{0} / \eta_{0}$ on the non-dimensional shear rate $\hat{\gamma}=\theta_{0} \dot{\gamma}$, presented as temperature independent "master curves".

Figure 5.18 shows master curves as double logarithmic plots for (a) $\hat{\eta}$ versus $\hat{\dot{\gamma}}$, and (b) $\hat{N}_{1}$ versus $\hat{\dot{\gamma}}$ for both experimental data (symbols) and fitting curves (lines) for steady shearing of PSHQ9 in the nematic region at three different temperatures. The fitting curves are represented as master curves in both (a) and (b). The large difference between experimental data and fitted theoretical master curves could be caused by the fact that unlike our assumption, the anisotropic constitutive parameters $r_{1}, r_{2}, \lambda_{e}, \lambda_{v}, \hat{a}_{1}$, and $\hat{\beta}$ may be temperature dependent.

Figure 5.19 shows master curves as double logarithmic plots for (a) $\hat{\eta}$ versus $\hat{\dot{\gamma}}$, and (b) $\hat{N}_{1}$ versus $\hat{\dot{\gamma}}$ for both experimental data (symbols) and fitting curves (lines) for steady 
shearing of PI-14-5CN in the nematic region at three different temperatures. It is seen that the fitting curves at different temperatures are represented as unique master curves for both (a) and (b) cases. The differences between the master curves of fitting results and experimental data arise from relatively low precision during the steady fitting procedure.
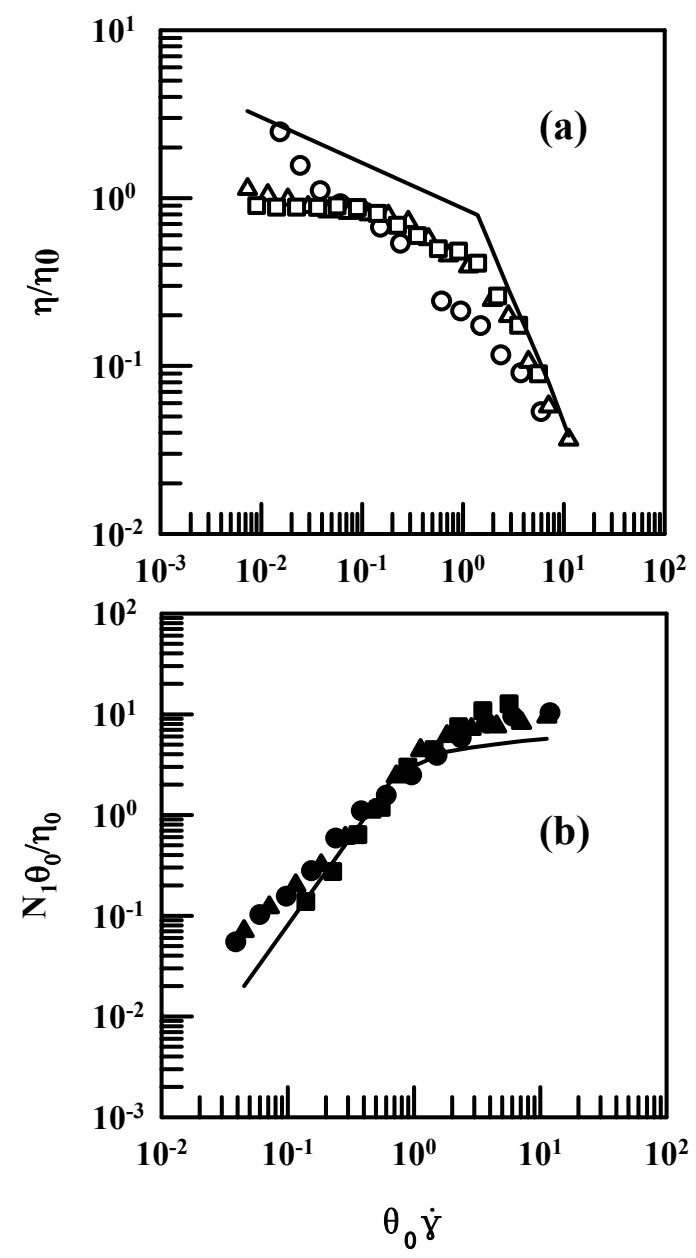

Figure 5.18 Master curves of experimental data (a) $\log \hat{\eta}=\eta / \eta_{0}$ vs $\log \hat{\dot{\gamma}}=\theta_{0} \dot{\gamma} \quad$ (open symbols) and (b) $\log \hat{N}_{1}=N_{1} \theta_{0} / \eta_{0}$ vs $\log \hat{\dot{\gamma}}=\theta_{0} \dot{\gamma}$ (filled symbols) for PSHQ9 in steady flow at different temperatures in the nematic region: $(\circ, \bullet) 130{ }^{\circ} \mathrm{C} ;(\Delta, \boldsymbol{\Delta}) 140^{\circ} \mathrm{C} ;(\square, \boldsymbol{\square})$ $150^{\circ} \mathrm{C}$. Corresponding fitted theoretical master curves at different temperatures are shown by solid lines. 

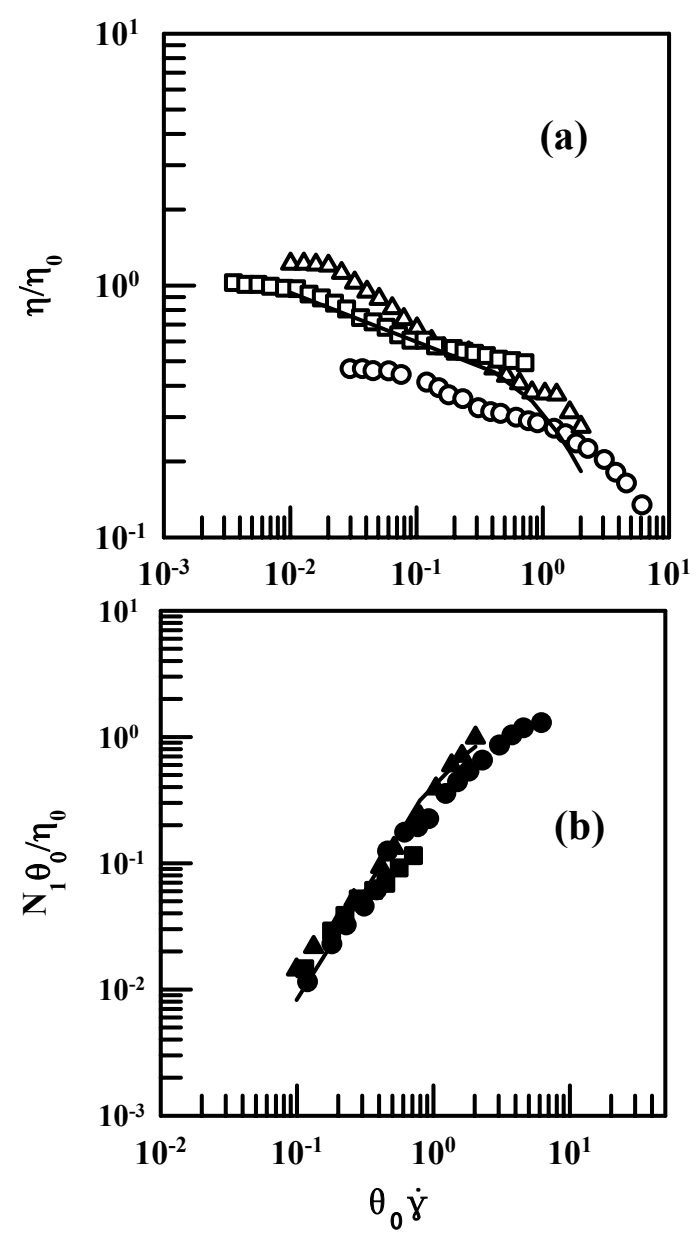

Figure 5.19 Master curves and experimental data for (a) $\log \hat{\eta}=\eta / \eta_{0}$ vs $\log \hat{\dot{\gamma}}=\theta_{0} \dot{\gamma}$ (open symbols) and (b) $\log \hat{N}_{1}=N_{1} \theta_{0} / \eta_{0}$ vs $\log \hat{\dot{\gamma}}=\theta_{0} \dot{\gamma}$ (filled symbols) for PI-14-5CN in steady shearing at different temperatures in the nematic region: $(\circ, \bullet) 70{ }^{\circ} \mathrm{C} ;(\Delta, \boldsymbol{\Delta}) 80{ }^{\circ} \mathrm{C}$; $(\square, \boldsymbol{\square}) 90^{\circ} \mathrm{C}$. Corresponding fitted theoretical master curves at different temperatures are shown by solid lines.

It should be mentioned that the deviation of calculated master curves from experimental data for steady shearing have the same precision as those shown for different temperatures in Figures $5.18 \& 5.19$, under the same (generally arbitrary) simplifying assumption that the above parameters of anisotropy are temperature independent. Although the better fitting for steady shearing could be achieved, one 
should recall that the established set of fitting parameters has been used not only for simulations of steady flows but also for such unsteady shearing as start up flow and relaxation. 


\section{CHAPTER VI}

\section{CONCLUSIONS}

The objective of this work was to analytically and numerically describe the rheological properties of thermotropic LCP's, using recently developed thermodynamic theory of weakly viscoelastic nematodynamics [12-14]. The current available theories such as Leslie-Ericksen theory and Doi theory for LCP's are not suitable for description of rheological behavior of thermotropic LCP's. Therefore, using these theories is very difficult to predict the rheological behaviors of LCP's. In contrast, viscoelastic nematodyanmics paves a new approach to circumvent this problem.

Based on the assumption of smallness of elastic (transient) strains and elastic relative rotation, the approach [12-14] makes significant contribution to theory of LCP's. The new model of weak viscoelastic nematodynamics combines the de Gennes-type elastic potential and LEP viscous nematodynamic approach in one constitutive equation, while ignoring Frank elasticity and inertia effects. In the absence of magnetic field, the theory exhibits viscoelastic transversally anisotropic behavior with symmetric stress tensor and orientation of director caused only by flow. Thus, this simplified approach has led to a closed set of two coupled anisotropic viscoelastic equations for evolution of director and extra stress.

Particularly, this theory is modeled by a set of quasi-linear anisotropic viscoelastic constitutive equations (CE) with anisotropy described by director, whose viscoelastic 
evolution is coupled with the CE. In spite of the fact that this theory has been developed for low enough value of Deborah number, it is still possible to compare the simulations with experimental data. A new mathematical tool, algebra of nematic operators, is helpful in operations with this multi-parametric theory. Eight parameters are in the theory which includes 3 viscosities, 3 elastic moduli, and 2 tumbling (elastic and viscous) parameters. These constitutive parameters established for steady shearing are used for the calculations of evolution of shear stress and first normal stress difference with corresponding evolution of director, during relaxation and start up flow. The problem with initial conditions for director in start up flow is resolved in the following way. We preliminarily fitted the experimental data for stresses in steady shearing with following adjustment of parameters to describe also the relaxation of stresses. In this case the parameters of evolution equation for director, along with its orientation in steady shearing were also established. The orientation of director during stress relaxation was then easily calculated, and its final orientation at the rest state was taken as initial one for the start up flow.

We judiciously chose the rheological data including steady flow, transient shear flow, and relaxation upon cessation of shear flow for two industrial LCP's, Titan and Zenite 6000, as well as two academic LCP's, a main-chain LCP (PSHQ9) and a sidechain LCP (PI-14-5CN). We demonstrate that our simulations are at least in a semiquantitative agreement with these experimental data, which is highly dependent on the structure of LCP's. Specifically, Titan is random copolyester of ethylene-terephthalate (PET) and hydroxybenzoic acid (HNA) with two methylene flexible spacers, whereas Zenite 6000 is a fully aromatic copolyester with kinks, Zenite 6000 is much more rigid than Titan. The absence of region I in the $\log \eta$ vs $\log \dot{\gamma}$ plots might be due to the fact 
that PI-14-5CN is a side-chain LCP, and its nematicity might not be sufficiently strong. The mesongens in the main-chain LCP PSHQ9 are directly linked to the polymer backbone, making motions of the mesogens and backbone of PSHQ9 strongly coupled during shear flow.

Finally, it seems that the possible reasons for several deviations of simulations from the experimental data are caused by the implementation of the monodomain approach to polydomain experimental data. 


\section{REFERENCES}

1. Collings, P. J.; Hird, M. Introduction to Liquid Crystals Chemistry and Physics. Taylor and Francis: London, 1997.

2. Donald, A. M.; Windle, A. H. Liquid Crystalline Polymers. Donald, A. M. and Windle, A. H., Ed.. University Press: Cambridge, 1992.

3. Chandrasekhar, S. Liquid Crystals, 2nd Ed. ed. Cambridge University Press: New York, 1992.

4. Dierking, I. Textures of Liquid Crystals. Wiley-VCH: Weinheim, 2003.

5. de Gennes, P. G.; Prost, J. The Physics of Liquid Crystals, 2nd Ed. ed. Clarendon Press: Oxford, 1993.

6. Warner, M.; Terentjev, E. M. Liquid Crystal Elastomers. Clarendon Press: Oxford, 2003.

7. Larson, R. G. The Structure and Rheology of Complex Fluids. Oxford Press: New York, 1999.

8. Golubovich, L.; Lubensky, T. C. Nonlinear elasticity of amorphous solids. Physical Review Letters 1989, 63, 1082-5.

9. Lubensky, T. C.; Mukhopadya, R. Symmetries and elasticity of nematic gels. Physical Review E 2002, 66, 011702.

10. Parodi, O. Stress tensor for a nematic liquid crystal. Journal de Physique France 1970, 31, 581-4.

11. de Gennes, P. G. Weak Nematic Gels. Helfich, W. and Kleppke, G., Ed.; Springer: Berlin, 1980.

12. Leonov, A. I. A model of weak viscoelastic nematodynamics. e-print: http://arxiv.org/abs/cond-mat/0507281,2005; Zeitschr. Angew. Math. Phys., 2008, 59, 333-359. 
13. Leonov, A.I., "Algebraic theory of linear viscoelastic nematodynamics", submitted to Mathematical Physics, Analysis and Geometry. See also: Algebraic theory of linear viscoelastic nematodynamics, Part 1: Algebra of nematic operators, http://arxiv.org/e-print/cond-mat/0409274; Part 2, Linear nematic viscoelasticity . (2004), http://arxiv.org/e-print/cond-mat/0409275. (2004)

14. Leonov, A. I.; Volkov, V. S. Weakly nonlinear viscoelastic nematodynamics. Journal of Engineering Physics and Thermophysics 2003, 76, 498-506.

15. Khoo, I.-C. Liquid Crystals, 2nd Ed. ed. Wiley-Interscience: Hoboken, N.J., 2007.

16. Wang, X.-J.; Zhou, Q.-F. Liquid Crystalline Polymers. World Scientific Pub. Co.: River Edge, N.J., 2004.

17. Yamakawa, H. Modern Theory of Polymer Solutions. Harper and Row: New York, 1971.

18. Flory, P. J. Statistical thermodynamics of mixtures of rodlike particles. 6. Rods connected by flexible joints. Macromolecules 1978, 11, 1141-4.

19. Black, W. B.; Preston, J. High-Modulus Wholly Aromatic Fibers; Marcel Dekker: New York, 1973.

20. Black, W. B. Flow-induced Crystallization in Polymer Systems. Miller, R. L., Ed. Gordon Breach: New York, 1979.

21. Morgan, P. W. Synthesis and Properties of Aromatic and Extended Chain Polyamides. Macromolecules 1977, 10, 1381-1390.

22. Jackson, W. J.; Kuhfuss, H. F. Liquid crystal polymers. I. Preparation and properties of p-hydroxybenzoic acid copolyesters. Journal of Polymer Science, Polymer Chemistry 1976, 14, 2043-58.

23. Furukawa, A.; Lenz, R. W. Liquid-crystal polymers. 23. Steric and polar effects of large substituents in thermotropic aromatic polyesters with decamethylene spacers. Makromolekulare Chemie, Macromolecular Symposia 1986, 2, 3-20.

24. Huang, W.; Han, C. D. Synthesis of combined main-chain/side-chain liquidcrystalline polymers via self-assembly. Macromolecules 2006, 39, 4735-45.

25. Kiss, G.; Porter, R. S. Rheology of concentrated solution of poly $(\gamma$-benzylglutamate). Journal of Polymer Science, Polymer Symposia 1978, 65, 193-211.

26. Kiss, G.; Porter, R. S. Rheology of concentrated solution of helical polypeptides. Journal of Polymer Science, Polymer Physics Edition 1980, 18, 361-88. 
27. Kiss, G.; Porter, R. S. Rheooptical studies of liquid crystalline solutions of helical polypeptides. Molecular Crystals and Liquid Crystals 1980, 60, 267-80.

28. Asada, T.; Muramatsu, H.; Watanabe, R.; Onogi, S. Rheooptical studies of racemic poly( $\gamma$-benzyl glutamate) liquid crystals. Macromolecules 1980, 13, 867-71.

29. Moldenaers, P.; Mewis, J. Transient behavior of liquid crystalline solutions of poly(benzyl glutamate). Journal of Rheology 1986, 30, 567-84.

30. Mewis, J.; Moldenaers, P. Transient rheological behavior of a lyotropic polymeric liquid crystal. Molecular Crystals and Liquid Crystals 1987, 153, 291-300.

31. Mead, D. W.; Larson, R. G. Rheooptical study of isotropic solutions of stiff polymers. Macromolecles 1990, 23, 2524-33.

32. Magda, J. J.; Baek, S. G.; DeVries, K. L.; Larson, R. G. Shear flows of liquid crystal polymers: measurements of the second normal stress difference and the Doi molecular theory. Macromolecules 1991, 24, 4460-8.

33. Baek, S. G.; Magda, J. J.; Cementwala, S. Normal stress differences in liquidcrystalline hydroxypropyl cellulos solutions. Journal of Rheology 1993, 37, 935-45.

34. Walker, L. M.; Wagner, N. J.; Larson, R. G.; Mirau, P. A.; Moldenaers, P. The rheology of highly concentrated PBLG solution. Journal of Rheology 1995, 39, $925-$ 52.

35. Navard, P.; Haudin, J. M. Rheology of hydroxypropyl cellulose solutions. Journal of Polymer Science, Polymer Physics Edition 1986, 24, 189-201.

36. Baek, S. G.; Magda, J. J.; Larson, R. G.; Hudson, S. D. Rheological differences among liquid-crystalline polymers. II. Disapperance of negative N1 in densely packed lyotropes and thermotropes. Journal of Rheology 1994, 38, 1473-503.

37. Hongladarom, K.; Secakusuma, V.; Burghardt, W. R. Relation between molecular orientation and rheology in lyotropic hydroxypropyl cellulose solutions. Journal of Rheology 1994, 38, 1505-23.

38. Hongladarom, K.; Ugaz, V. M.; Cinader, D. K.; Burghardt, W. R.; Quintana, J. P.; Hsiao, B. S.; Dadmun, M. D.; Hamilton, W. A.; Butler, P. D. Birefringence, X-ray scattering, and neutron scattering measurements of molecular orientation in sheared liquid crystal polymer solutions. Macromolecules 1996, 29, 5346-55.

39. Huang, C.-M.; Magda, J. J.; Larson, R. G. The effect of temperature and concentration on $\mathrm{N} 1$ and tumbling in a liquid crystal polymer. Journal of Rheology 1999, 43, 31-50. 
40. Valenti, B.; Ciferri, A. Rheological behavior of an aromatic polyamide-hydrazide. Journal of polymer science, Polymer Letters Edition 1978, 16, 657-63.

41. Wong, C. P.; Ohnuma, H.; Berry, G. C. Properties of some rodlike polymers in solution. Journal of Polymer Science, Polymer Symposia 1978, 65, 173-92.

42. Einaga, Y.; Berry, G. C.; Chu, S. G. Rheological properties of rodlike polymers in solution. III. Transient and steady-state studies on nematic solutions. Polymer Journal 1985, 17, 239-51.

43. Wissbrun, K. F.; Griffin, A. C. Rheology of a thermotropic polyester in the nematic and isotropic states. Journal of Polymer Science, Polymer Physics Edition 1982, 20, $1835-45$.

44. Wunder, S. L.; Ramachandran, S.; Gochanour, C. R.; Weinberg, M. Rheology of phase separation in anisotropic melt polymers. Macromolecules 1986, 19, 1696-702.

45. Irwin, R. S.; Sweeny, W.; Gardner, K. H.; Gochanour, C. R.; Weinberg, M. 3,4'Dihydroxybenzophenone terephthalate: an all-aromatic thermotropic polyester with a helical chain conformation. Macromolecules 1989, 22, 1065-74.

46. Driscoll, P.; Masuda, T.; Fujiwara, K. Rheological properties of a homogeneous thermotropic liquid-crystalline polyester: dynamic viscoelastic and interrupted-flow measurements. Macromolecules 1991, 24, 1567-74.

47. Cocchini, F.; Nobile, M. R.; Acierno, D. Transient and steady rheological behavior of the thermotropic liquid crystal copolymer 73/27 HBA/HNA. Journal of Rheology 1991, 35, 1171-89.

48. Kim, S. S.; Han, C. D. Effect of molecular weight on the rheological behaviro of thermotropic liquid-crystalline polymer. Macromolecules 1993, 26, 6633-42.

49. Kim, S. S.; Han, C. D. Effect of shear history on the steady shear flow behavior of a thermotropic liquid-crystalline polymer. Journal of Polymer Science, Part B:

Polymer Physics 1994, 32, 371-81.

50. Han, C. D.; Chang, S.; Kim, S. S. Rheological behavior of thermotropic liquidcrystalline polymers: effects of thermal and deformation histories. Molecular Crystals and Liquid Crystals Science and Technology, Section A: Molecular Crystals and Liquid Crystals. 1994, 254, 335-68.

51. Chang, S.; Han, C. D. Effect of flexible spacers on the rheological behavior of mainchain thermotropic liquid-crystalline polymers having bulky pendent side groups. Macromolecules 1997, 30, 2021-34. 
52. Chang, S.; Han, C. D. A thermotropic main-chain random copolyester containing flexible spacers of differing lengths. 2. rheological behavior. Macromolecules 1997, 30, 1656-69.

53. Zhou, W.-J.; Kornfield, J. A.; Ugaz, V. M.; Burghardt, W. R.; Link, D. R.; Clark, N. A. Dynamics and shear orientation behavior of a main-chain thermotropic liquid crystalline polymer. Macromolecules 1999, 32, 5581-93.

54. Zentel, R.; Wu, J. Rheological properties of liquid-crystalline side-group polymers in the isotropic, nematic, and smectic states. Makromolekulare Chemie 1986, 187, $1727-36$.

55. Colby, R. H.; Gillmor, J. R.; Galli, G.; Laus, M.; Ober, C. K.; Hall, E. Linear viscoelasticity of side chain liquid crystal polymers. Liquid Crystals 1993, 13, 23345.

56. Berghausen, J.; Fuchs, J.; Richtering, W. Rheology and shear orientation of nematic liquid crystalline side-group polymer with laterally attached mesogenic units. Macromolecules 1997, 30, 7574-81.

57. Quijada-Garrido, I.; Siebert, H.; Friedrich, C.; Schmidt, C. Flow behavior of two side-chain liquid crystal polymers, studied by transient rheology. Macromolecules 2000, 33, 3844-54.

58. Wewerka, A.; Floudas, G.; Pakula, T.; Stelzer, F. Side-chain liquid crystalline homopolymers and copolymers. Structure and rheology. Macromolecules 2001, 34, 8129-37.

59. Lee, K. M.; Han, C. D. Rheology of nematic side-chain liquid-crystalline polymer: comparison with main-chain liquid-crystalline polymer. Macromolecules 2002, 35, 6263-73.

60. Lee, K. M.; Han, C. D. Effect of flexible spacer length on the rheology of side-chain liquid crystalline polymers. Macromolecules 2003, 36, 8796-810.

61. Zhou, M.; Han, C. D. Rheology of a combined main-chain/side-chain liquidcrystalline polymer in the thermotropic and lyotropic states. Macromolecules 2006, $39,232-42$.

62. Kim, S. S.; Han, C. D. Effect of thermal history on the rheological behavior of a thermotropic liquid-crystalline polymer. Macromolecules 1993, 26, 3176-86.

63. Belatreche, M. J. An Experimental and Theoretical Study of Liquid Crystalline Polymers. The University of Akron: Akron, 2002. 
64. Onogi, T. A. Proceedings of the Seventh International Congress on Rheology. Astarita, G., Marrucci, G. and Nicolais, L., Ed.; Plenum Press: New York, 1980; Vol. Vol I, pp 127.

65. Marrucci, G.; Maffettone, P. L. Liquid Crystalline Polymers, Carfagna, C., Ed.; Pergamon: New York, 1993.

66. Burghardt, W. R.; Hongladarom, K. Texture refinement in a sheared liquidcrystalline polymer. Macromolecules 1994, 27, 2327-9.

67. Marrucci, G.; Maffettone, P. L. Nematic phase of rodlike polymers. I. Prediction of transient behavior at high shear rates. Journal of Rheology 1990, 34, 1217-30.

68. Marrucci, G.; Maffettone, P. L. Description of the liquid-crystalline phase of rodlike polymers at high shear rates. Macromolecules 1989, 22, 4076-82.

69. Larson, R. G. Arrested tumbling in shearing flow of liquid-crystal polymers. Macromolecules 1990, 23, 3983-92.

70. Doi, M. Molecular dynamics and rheological properties of concentrated solutions of rodlike polymers in isotropic and liquid crystalline phases. Journal of Polymer Science: Polymer Physics Edition 1981, 19, 229-43.

71. Doi, M.; Edwards, S. F. S. Dynamics of rod-like macromolecules in concentrated solution. Part 2. Journal of the Chemical Society, Faraday Transactions 2:

Molecular and Chemical Physics 1978, 74, 918-32.

72. Murrucci, G.; Maffettone, P. L. A description of the liquid-crytalline phase of rodlike polymers at high shear rates. Macromolecules 1989, 22, 4076-82.

73. Ericksen, J. L. Anisotropic fluids. Archive for Rational Mechanics and Analysis 1960, 4, 231-7.

74. Leslie, F. M. Some constitutive equations for liquid crystals. Archive for Rational Mechanics and Analysis 1968, 28, 265-83.

75. Leslie, F. M. Some constitutive equations for anisotropic fluids. The Quarterly Journal of Mechanics and Applied Mathematics 1966, 19, 357-70.

76. Leslie, F. M. Advances in Liquid Crystals, Brown, G. H., Ed. Academic Press: New York, 1979, pp 1.

77. de Gennes, P. G. Viscometric flow of tangled polymers. Comptes Rendus des Seances de l'Academie des Sciences, Serie B: Sciences Physiques 1979, 288, 219-20. 
78. Doi, M.; Edwards, S. F. Dynamics of rod-like macromolecules in concentrated solution. Part 1. Journal of the Chemical Society, Faraday Transactions 2:

Molecular and Chemical Physics 1978, 74, 560-70.

79. Doi, M. Rheological properties of rodlike polymers in isotropic and liquid crystalline phases. Ferroelectric 1980, 30, 247-54.

80. Doi, M.; Edwards, S. F. The Theory of Polymer Dynamics. Oxford University Press: New York, 1986.

81. Larson, R. G.; Doi, M. Mesoscopic domain theory for textured liquid crystalline polymers. Journal of Rheology 1991, 35, 539-63.

82. Marrucci, G. Rheology of liquid crystalline polymers. Pure and Applied Chemistry 1985, 57, 1545-52.

83. Kirkwood, J. G.; Auer, P. L. The visco-elastic properties of solutions of rod-like macromolecules. The Journal of Chemical Physics 1951, 19, 281-3.

84. Guenther, G. K.; Baird, D. G.; Davis, R. M. Rheological properties of poly-pphenyleneterephatalamide $/ \mathrm{H}_{2} \mathrm{SO}_{4}$ solutions; Carfagne, C., Ed. Elsevier: Capri, Italy, 1993, pp 133-42.

85. Odell, J. A.; Ungar, G.; Feijoo, J. L. A rheological, optical, and X-ray study of the relaxation of orientation of nematic PBZT. Journal of Polymer Science, Part B: Polymer Physics 1993, 31, 141-55.

86. Ernst, B.; Denn, M. M.; Pierini, P.; Rochefort, W. E. Rheological properties of liquid crystalline solutions of cis-poly(p-phenylenebenzobisoxazole) in polyphosphoric acid (PBO/PPA). Journal of Rheology 1992, 36, 289-302.

87. de Gennes, P. G. Short-range order effects in the isotropic phase of nematics and cholesterics. Molecular Crystals and Liquid Crystals 1971, 12, 193-214.

88. Larson, R. G.; Mead, D. W. Linear viscoelasticity of nematic liquid-crystalline polymers. Journal of Rheology 1989, 33, 185-206.

89. Doi, M. Molecular Theory for the Nonlinear Viscoelasticity of Polymeric Liquid Crystals; Ericksen, J. L. and Kinderlehrer, D., Ed. Springer-Verlag: New York, 1987.

90. Volkov, V. S.; Kulichikhin, V. G. Anisotropic viscoelasticity of liquid crystalline polymers. Journal of Rheology 1990, 34, 281-93. 
91. Pleiner, H.; Brand, H. R. Macroscopic dynamic equations for nematic liquid crystalline side-chain polymers. Molecular Crystals and Liquid Crystals 1991, 199, $407-18$.

92. Pleiner, H.; Brand, H. R. Local rotational degrees of freedom in nematic liquidcrystalline side-chain polymers. Macromolecules 1992, 25, 895-901.

93. Rey, A. D. Anisotropic viscoelasticity of side chain nematic polymers. Journal of Non-Newtonian Fluid Mechanics 1995, 58, 131-60.

94. Rey, R. D. Macroscopic theory of orientation transitions in the extensional flow of side-chain nematic polymers. Rheologica Acta 1995, 34, 119-31.

95. Terentjev, E. M.; Warner, M. Linear hydrodynamics and viscoelasticity of nematic elastomers. The European Physical Journal E 2001, 4, 343-53.

96. Leonov, A. I.; Volkov, V. S. Prog. 6th Europ. Conf. on Rheology, Munstedt, H., Kaschta, J. and Merten, A., Ed. Erlangen, Germany, 2002.

97. Leonov, A. I.; Volkov, V. S. Internal rotations and stress tensor symmetry in theories of nematic liquids and solids. e-print: cond.mat/0203265, 2002.

98. Marrucci, G.; Greco, F. Flow behavior of liquid crystalline polymers. Advances in Chemical Physics 1993, 86, 331-404.

99. Feng, J. J.; Sgalari, G.; Leal, L. G. A theory for flowing nematic polymers with orientatinal distortion. Journal of Rheology 2000, 44, 1085-101.

100. Edwards, B. J.; Beris, A. N. Generalized constitutive equation for polymeric liquid crystals Part 1. Model formulation using the Hamiltonian (poisson brackket) formulation. Journal of Non-Newtonian Fluid Mechanics 1990, 35, 51-72.

101. Beris, A. N.; Edwards, B. J. Thermodynamics of Flowing Systems. Oxford University Press: Oxford, 1999.

102. Volkov, V. S.; Kulichikhin, V. G. Macromolecular dynamics in anisotropic viscoelastic liquids. Macromolecular Symposia (Statistical Mechanics of Condensed Polymer Systems: Theory and Simulations) 1994, 81, 45-53.

103. Long, D.; Morse, D. C. A Rouse-like model of liquid crystalline polymer melts: Director dynamics and linear viscoelasticity. Journal of Rheology 2002, 46, 49-92.

104. Golubovic, L.; Lubensky, T. C. Nonlinear elasticity of amorphous solids. Physical Review Letters 1989, 63, 1082-5. 
105. Olmsted, P. D. Rotational invariance and goldstone modes in nematic elastomers and gels. Journal de Physique II France 1994, 4, 2215-30.

106. Leonov, A. I.; Volkov, V. S. General analysis of linear nematic elasticity. Journal of Engineering Physics and Thermophysics 2004, 77, 717-26.

107. Leonov, A. I.; Volkov, V. S. Dissipative soft modes in viscous nematodynamics. Rheologica Acta 2005, 44, 331-41.

108. Leonov, A. I. On the minimum of extended dissipation in viscous nematodynamics. Rheologica Acta 2005, 44, 573-6.

109. Han, C. D. Rheology and Processing of Polymeric Materials. Oxford University Press: New York, 2007; Vol. I. 


\section{APPENDIX}

A-1. Using Mathematica, the equations (5-1.a-c) were analytically solved, and then $\sigma_{11}$, $\sigma_{12}, \sigma_{22}, \sigma_{33}, N_{1}$, and $N_{2}$ were expressed in terms of the constitutive parameters, $\theta_{0}$,

$\dot{\gamma}, r_{1}, r_{2}, n_{1}, n_{2}, \eta_{0}, \hat{a}_{1}$, and $\hat{\beta}$

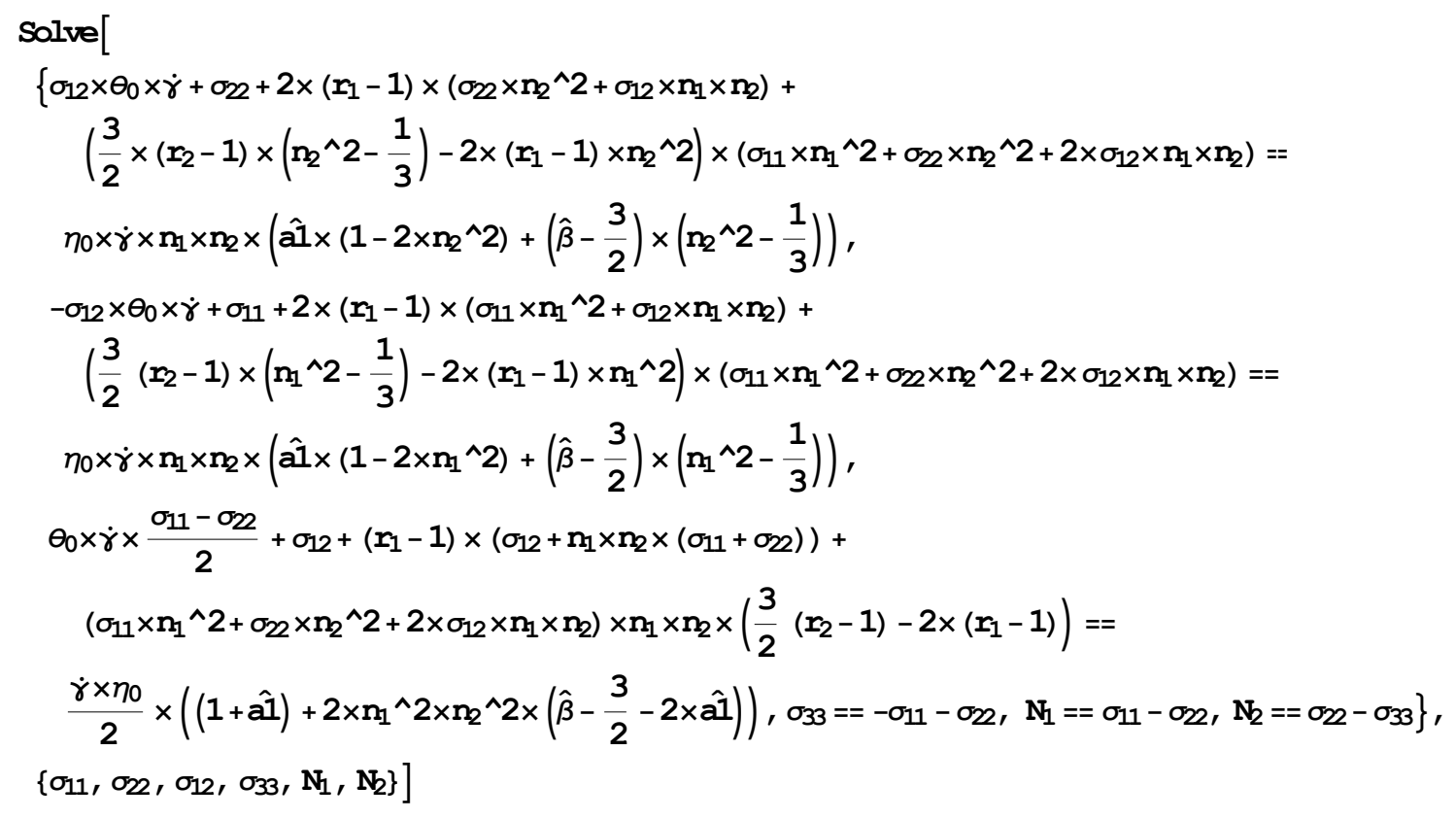

Then the solutions of $\sigma_{11}, \sigma_{12}, \sigma_{22}, \sigma_{33}, N_{1}$, and $N_{2}$ are given as follows: 
$-\dot{\gamma} \eta_{0}-\dot{\gamma} \hat{a} \eta_{0}+3 \dot{\gamma} n_{1}^{2} n_{2}^{2} \eta_{0}+4 \dot{\gamma} \hat{a} n_{1}^{2} n_{2}^{2} \eta_{0}-2 \dot{\gamma} \hat{\beta} n_{1}^{2} n_{2}^{2} \eta_{0}$

$-2 n_{1} n_{2}+n_{1}^{3} n_{2}+2 n_{1} n_{2} r_{1}-4 n_{1}^{3} n_{2} r_{1}+3 n_{1}^{3} n_{2} r_{2}+\dot{\gamma} \theta_{0}$

$\left(2\left(6 \dot{\gamma} \eta_{0}+6 \dot{\gamma} \hat{a} \eta_{0}-9 \dot{\gamma} n_{1}^{2} \eta_{0}-9 \dot{\gamma}\right.\right.$ âl $n_{1}^{2} \eta_{0}+3 \dot{\gamma} n_{1}^{4} \eta_{0}+3 \dot{\gamma} \hat{a} \hat{1} n_{1}^{4} \eta_{0}-12 \dot{\gamma} n_{1}^{2} n_{2}^{2} \eta_{0}-12 \dot{\gamma} a \hat{1} n_{1}^{2} n_{2}^{2} \eta_{0}+$ $8 \dot{\gamma} \hat{\beta} n_{1}^{2} n_{2}^{2} \eta_{0}+6 \dot{\gamma} n_{1}^{4} n_{2}^{2} \eta_{0}+6 \dot{\gamma} \hat{a} \hat{l} n_{1}^{4} n_{2}^{2} \eta_{0}-4 \dot{\gamma} \hat{\beta} n_{1}^{4} n_{2}^{2} \eta_{0}+12 \dot{\gamma} n_{1}^{2} n_{1} \eta_{0}+12 \dot{\gamma} \hat{a} n_{1}^{2} n_{1} \eta_{0}-$

$12 \dot{\gamma} n_{1}^{4} r_{1} \eta_{0}-12 \dot{\gamma}$ al $n_{1}^{4} r_{1} \eta_{0}-6 \dot{\gamma} n_{1}^{2} n_{2}^{2} r_{1} \eta_{0}-12 \dot{\gamma}$ âl $n_{1}^{2} n_{2}^{2} r_{1} \eta_{0}+4 \dot{\gamma} \hat{\beta} n_{1}^{2} n_{2}^{2} r_{1} \eta_{0}-6 \dot{\gamma} n_{1}^{4} n_{2}^{2} r_{1} \eta_{0}+$

$4 \dot{\gamma} \hat{\beta} n_{1}^{4} n_{2}^{2} r_{1} \eta_{0}-3 \dot{\gamma} n_{1}^{2} r_{2} \eta_{0}-3 \dot{\gamma} a \hat{l} n_{1}^{2} r_{2} \eta_{0}+9 \dot{\gamma} n_{1}^{4} r_{2} \eta_{0}+9 \dot{\gamma}$ al $n_{1}^{4} r_{2} \eta_{0}-6 \dot{\gamma} a 1 n_{1}^{4} n_{2}^{2} r_{2} \eta_{0}-$

$\left.\left.3 \dot{\gamma}^{2} n_{1} n_{2} \eta_{0} \theta_{0}-6 \dot{\gamma}^{2} \hat{a} n_{1} n_{2} \eta_{0} \theta_{0}+2 \dot{\gamma}^{2} \hat{\beta} n_{1} n_{2} \eta_{0} \theta_{0}+9 \dot{\gamma}^{2} n_{1}^{3} n_{2} \eta_{0} \theta_{0}+12 \dot{\gamma}^{2} \hat{a} n_{1}^{3} n_{2} \eta_{0} \theta_{0}-6 \dot{\gamma}^{2} \hat{\beta} n_{1}^{3} n_{2} \eta_{0} \theta_{0}\right)\right) /$

$\left(12 n_{1} n_{2}-18 n_{1}^{3} n_{2}+6 n_{1}^{5} n_{2}-12 n_{1} n_{2}^{3}+6 n_{1}^{3} n_{2}^{3}-12 n_{1} n_{2} r_{1}+42 n_{1}^{3} n_{2} r_{1}-30 n_{1}^{5} n_{2} r_{1}+30 n_{1} n_{2}^{3} r_{1}-\right.$

$30 n_{1}^{3} n_{2}^{3} n_{1}-24 n_{1}^{3} n_{2} r_{1}^{2}+24 n_{1}^{5} n_{2} r_{1}^{2}+24 n_{1}^{3} n_{2}^{3} r_{1}^{2}-6 n_{1}^{3} n_{2} r_{2}+18 n_{1}^{5} n_{2} r_{2}-12 n_{1} n_{2}^{3} r_{2}+18 n_{1}^{3} n_{2}^{3} r_{2}+$

$6 n_{1}^{3} n_{2} r_{1} r_{2}-18 n_{1}^{5} n_{2} r_{1} r_{2}-6 n_{1} n_{2}^{3} r_{1} r_{2}-18 n_{1}^{3} n_{2}^{3} r_{1} r_{2}+6 \dot{\gamma} \theta_{0}-9 \dot{\gamma} n_{1}^{2} \theta_{0}+3 \dot{\gamma} n_{1}^{4} \theta_{0}+3 \dot{\gamma} n_{2}^{2} \theta_{0}+$

$\left.3 \dot{\gamma} n_{1}^{2} n_{2}^{2} \theta_{0}+12 \dot{\gamma} n_{1}^{2} r_{1} \theta_{0}-12 \dot{\gamma} n_{1}^{4} r_{1} \theta_{0}-12 \dot{\gamma} n_{1}^{2} n_{2}^{2} r_{1} \theta_{0}-3 \dot{\gamma} n_{1}^{2} r_{2} \theta_{0}+9 \dot{\gamma} n_{1}^{4} r_{2} \theta_{0}-3 \dot{\gamma} n_{2}^{2} r_{2} \theta_{0}+9 \dot{\gamma} n_{1}^{2} n_{2}^{2} r_{2} \theta_{0}\right)+$

$\left(\left(2 n_{1}^{2} n_{2}^{2}+2 r_{1}-8 n_{1}^{2} n_{2}^{2} r_{1}+6 n_{1}^{2} n_{2}^{2} r_{2}\right)\right.$

$\left(-36 \dot{\gamma} \eta_{0}-36 \dot{\gamma}\right.$ all $\eta_{0}+54 \dot{\gamma} n_{1}^{2} \eta_{0}+54 \dot{\gamma}$ al $n_{1}^{2} \eta_{0}-18 \dot{\gamma} n_{1}^{4} \eta_{0}-18 \dot{\gamma}$ all $n_{1}^{4} \eta_{0}+54 \dot{\gamma} n_{2}^{2} \eta_{0}+54 \dot{\gamma}$ all $n_{2}^{2} \eta_{0}-$

$36 \dot{\gamma} n_{1}^{2} n_{2}^{2} \eta_{0}-72 \dot{\gamma} \hat{a} n_{1}^{2} n_{2}^{2} \eta_{0}-24 \dot{\gamma} \hat{\beta} n_{1}^{2} n_{2}^{2} \eta_{0}+72 \dot{\gamma} n_{1}^{4} n_{2}^{2} \eta_{0}+144 \dot{\gamma} \hat{a l} n_{1}^{4} n_{2}^{2} \eta_{0}-24 \dot{\gamma} \hat{\beta} n_{1}^{4} n_{2}^{2} \eta_{0}-$

$72 \dot{\gamma} n_{1}^{6} n_{2}^{2} \eta_{0}-108 \dot{\gamma} \hat{a} n_{1}^{6} n_{2}^{2} \eta_{0}+48 \dot{\gamma} \hat{\beta} n_{1}^{6} n_{2}^{2} \eta_{0}-18 \dot{\gamma} n_{2}^{4} \eta_{0}-18 \dot{\gamma} \hat{a} 1 n_{2}^{4} \eta_{0}+72 \dot{\gamma} n_{1}^{2} n_{2}^{4} \eta_{0}+$

$144 \dot{\gamma} \hat{a l} n_{1}^{2} n_{2}^{4} \eta_{0}-24 \dot{\gamma} \hat{\beta} n_{1}^{2} n_{2}^{4} \eta_{0}-144 \dot{\gamma} n_{1}^{4} n_{2}^{4} \eta_{0}-216 \dot{\gamma} \hat{a l} n_{1}^{4} n_{2}^{4} \eta_{0}+96 \dot{\gamma} \hat{\beta} n_{1}^{4} n_{2}^{4} \eta_{0}-72 \dot{\gamma} n_{1}^{2} n_{2}^{6} \eta_{0}-$

$108 \dot{\gamma} \hat{a} 1 n_{1}^{2} n_{2}^{6} \eta_{0}+48 \dot{\gamma} \hat{\beta} n_{1}^{2} n_{2}^{6} \eta_{0}-72 \dot{\gamma} n_{1}^{2} r_{1} \eta_{0}-72 \dot{\gamma} a 1 n_{1}^{2} r_{1} \eta_{0}+72 \dot{\gamma} n_{1}^{4} r_{1} \eta_{0}+72 \dot{\gamma} a l n_{1}^{4} r_{1} \eta_{0}-$

$72 \dot{\gamma} n_{2}^{2} r_{1} \eta_{0}-72 \dot{\gamma}$ al $n_{2}^{2} r_{1} \eta_{0}+288 \dot{\gamma} n_{1}^{2} n_{2}^{2} r_{1} \eta_{0}+360 \dot{\gamma}$ al $n_{1}^{2} n_{2}^{2} r_{1} \eta_{0}-48 \dot{\gamma} \hat{\beta} n_{1}^{2} n_{2}^{2} r_{1} \eta_{0}-$

$288 \dot{\gamma} n_{1}^{4} n_{2}^{2} r_{1} \eta_{0}-468 \dot{\gamma} a \hat{l} n_{1}^{4} n_{2}^{2} r_{1} \eta_{0}+72 \dot{\gamma} \hat{\beta} n_{1}^{4} n_{2}^{2} r_{1} \eta_{0}+144 \dot{\gamma} n_{1}^{6} n_{2}^{2} r_{1} \eta_{0}+252 \dot{\gamma}$ al $n_{1}^{6} n_{2}^{2} r_{1} \eta_{0}-$

$96 \dot{\gamma} \hat{\beta} n_{1}^{6} n_{2}^{2} r_{1} \eta_{0}+72 \dot{\gamma} n_{2}^{4} r_{1} \eta_{0}+72 \dot{\gamma}$ al $n_{2}^{4} r_{1} \eta_{0}-288 \dot{\gamma} n_{1}^{2} n_{2}^{4} r_{1} \eta_{0}-468 \dot{\gamma} a \hat{l} n_{1}^{2} n_{2}^{4} r_{1} \eta_{0}+$

$72 \dot{\gamma} \hat{\beta} n_{1}^{2} n_{2}^{4} r_{1} \eta_{0}+288 \dot{\gamma} n_{1}^{4} n_{2}^{4} r_{1} \eta_{0}+504 \dot{\gamma} \hat{a} n_{1}^{4} n_{2}^{4} r_{1} \eta_{0}-192 \dot{\gamma} \hat{\beta} n_{1}^{4} n_{2}^{4} r_{1} \eta_{0}+144 \dot{\gamma} n_{1}^{2} n_{2}^{6} r_{1} \eta_{0}+$

$252 \dot{\gamma} a 1 n_{1}^{2} n_{2}^{6} n_{1} \eta_{0}-96 \dot{\gamma} \hat{\beta} n_{1}^{2} n_{2}^{6} r_{1} \eta_{0}-144 \dot{\gamma} n_{1}^{2} n_{2}^{2} r_{1}^{2} \eta_{0}-144 \dot{\gamma} a 1 n_{1}^{2} n_{2}^{2} r_{1}^{2} \eta_{0}+216 \dot{\gamma} n_{1}^{4} n_{2}^{2} r_{1}^{2} \eta_{0}+$

$288 \dot{\gamma} \hat{a} 1 n_{1}^{4} n_{2}^{2} r_{1}^{2} \eta_{0}-48 \dot{\gamma} \hat{\beta} n_{1}^{4} n_{2}^{2} r_{1}^{2} \eta_{0}-72 \dot{\gamma} n_{1}^{6} n_{2}^{2} r_{1}^{2} \eta_{0}-144 \dot{\gamma}$ all $n_{1}^{6} n_{2}^{2} r_{1}^{2} \eta_{0}+48 \dot{\gamma} \hat{\beta} n_{1}^{6} n_{2}^{2} x_{1}^{2} \eta_{0}+$

$216 \dot{\gamma} n_{1}^{2} n_{2}^{4} r_{1}^{2} \eta_{0}+288 \dot{\gamma} a \hat{l} n_{1}^{2} n_{2}^{4} r_{1}^{2} \eta_{0}-48 \dot{\gamma} \hat{\beta} n_{1}^{2} n_{2}^{4} r_{1}^{2} \eta_{0}-144 \dot{\gamma} n_{1}^{4} n_{2}^{4} r_{1}^{2} \eta_{0}-288 \dot{\gamma}$ al $n_{1}^{4} n_{2}^{4} r_{1}^{2} \eta_{0}+$

$96 \dot{\gamma} \hat{\beta} n_{1}^{4} n_{2}^{4} r_{1}^{2} \eta_{0}-72 \dot{\gamma} n_{1}^{2} n_{2}^{6} r_{1}^{2} \eta_{0}-144 \dot{\gamma} \hat{a} n_{1}^{2} n_{2}^{6} r_{1}^{2} \eta_{0}+48 \dot{\gamma} \hat{\beta} n_{1}^{2} n_{2}^{6} r_{1}^{2} \eta_{0}+18 \dot{\gamma} n_{1}^{2} r_{2} \eta_{0}+$

$18 \dot{\gamma} \hat{a} 1 n_{1}^{2} r_{2} \eta_{0}-54 \dot{\gamma} n_{1}^{4} r_{2} \eta_{0}-54 \dot{\gamma} \hat{a} n_{1}^{4} r_{2} \eta_{0}+18 \dot{\gamma} n_{2}^{2} r_{2} \eta_{0}+18 \dot{\gamma} a \hat{l} n_{2}^{2} r_{2} \eta_{0}-72 \dot{\gamma} n_{1}^{2} n_{2}^{2} r_{2} \eta_{0}-$

$72 \dot{\gamma}$ all $n_{1}^{2} n_{2}^{2} r_{2} \eta_{0}+108 \dot{\gamma} n_{1}^{4} n_{2}^{2} r_{2} \eta_{0}+144 \dot{\gamma}$ all $n_{1}^{4} n_{2}^{2} r_{2} \eta_{0}-36 \dot{\gamma}$ al $n_{1}^{6} n_{2}^{2} r_{2} \eta_{0}-54 \dot{\gamma} n_{2}^{4} r_{2} \eta_{0}-$

$54 \dot{\gamma}$ all $n_{2}^{4} r_{2} \eta_{0}+108 \dot{\gamma} n_{1}^{2} n_{2}^{4} r_{2} \eta_{0}+144 \dot{\gamma}$ âl $n_{1}^{2} n_{2}^{4} r_{2} \eta_{0}-72 \dot{\gamma}$ all $n_{1}^{4} n_{2}^{4} r_{2} \eta_{0}-36 \dot{\gamma}$ âl $n_{1}^{2} n_{2}^{6} r_{2} \eta_{0}+$

$72 \dot{\gamma} n_{1}^{2} n_{2}^{2} r_{1} r_{2} \eta_{0}+72 \dot{\gamma} a 1 n_{1}^{2} n_{2}^{2} r_{1} r_{2} \eta_{0}-108 \dot{\gamma} n_{1}^{4} n_{2}^{2} r_{1} r_{2} \eta_{0}-108 \dot{\gamma}$ al $n_{1}^{4} n_{2}^{2} r_{1} r_{2} \eta_{0}+$

$36 \dot{\gamma}$ al $n_{1}^{6} n_{2}^{2} r_{1} r_{2} \eta_{0}-108 \dot{\gamma} n_{1}^{2} n_{2}^{4} r_{1} r_{2} \eta_{0}-108 \dot{\gamma}$ âl $n_{1}^{2} n_{2}^{4} r_{1} r_{2} \eta_{0}+72 \dot{\gamma}$ âl $n_{1}^{4} n_{2}^{4} r_{1} r_{2} \eta_{0}+$

$36 \dot{\gamma} \hat{a} n_{1}^{2} n_{2}^{6} r_{1} r_{2} \eta_{0}-18 \dot{\gamma}^{2} n_{1}^{3} n_{2} \eta_{0} \theta_{0}+12 \dot{\gamma}^{2} \hat{\beta} n_{1}^{3} n_{2} \eta_{0} \theta_{0}-36 \dot{\gamma}^{2} n_{1}^{5} n_{2} \eta_{0} \theta_{0}-54 \dot{\gamma}^{2} \hat{a} n_{1}^{5} n_{2} \eta_{0} \theta_{0}+$

$24 \dot{\gamma}^{2} \hat{\beta} n_{1}^{5} n_{2} \eta_{0} \theta_{0}+18 \dot{\gamma}^{2} n_{1} n_{2}^{3} \eta_{0} \theta_{0}-12 \dot{\gamma}^{2} \hat{\beta} n_{1} n_{2}^{3} \eta_{0} \theta_{0}+36 \dot{\gamma}^{2} n_{1} n_{2}^{5} \eta_{0} \theta_{0}+54 \dot{\gamma}^{2} \hat{a} 1 n_{1} n_{2}^{5} \eta_{0} \theta_{0}-$

$24 \dot{\gamma}^{2} \hat{\beta} n_{1} n_{2}^{5} \eta_{0} \theta_{0}-36 \dot{\gamma}^{2} n_{1}^{3} n_{2} r_{1} \eta_{0} \theta_{0}-72 \dot{\gamma}^{2} \hat{a} 1 n_{1}^{3} n_{2} r_{1} \eta_{0} \theta_{0}+24 \dot{\gamma}^{2} \hat{\beta} n_{1}^{3} n_{2} r_{1} \eta_{0} \theta_{0}+$

$36 \dot{\gamma}^{2} n_{1}^{5} n_{2} r_{1} \eta_{0} \theta_{0}+72 \dot{\gamma}^{2} \hat{a} 1 n_{1}^{5} n_{2} r_{1} \eta_{0} \theta_{0}-24 \dot{\gamma}^{2} \hat{\beta} n_{1}^{5} n_{2} r_{1} \eta_{0} \theta_{0}+36 \dot{\gamma}^{2} n_{1} n_{2}^{3} n_{1} \eta_{0} \theta_{0}+$

$72 \dot{\gamma}^{2} a 1 n_{1} n_{2}^{3} n_{1} \eta_{0} \theta_{0}-24 \dot{\gamma}^{2} \hat{\beta} n_{1} n_{2}^{3} n_{1} \eta_{0} \theta_{0}-36 \dot{\gamma}^{2} n_{1} n_{2}^{5} n_{1} \eta_{0} \theta_{0}-72 \dot{\gamma}^{2} \hat{a} 1 n_{1} n_{2}^{5} n_{1} \eta_{0} \theta_{0}+$

$\left.\left.24 \dot{\gamma}^{2} \hat{\beta} n_{1} n_{2}^{5} n_{1} \eta_{0} \theta_{0}-18 \dot{\gamma}^{2} \hat{a l} n_{1}^{5} n_{2} r_{2} \eta_{0} \theta_{0}+18 \dot{\gamma}^{2} \hat{l} n_{1} n_{2}^{5} r_{2} \eta_{0} \theta_{0}\right)\right) /$

$\left(\left(-2 n_{1} n_{2}+n_{1}^{3} n_{2}+2 n_{1} n_{2} r_{1}-4 n_{1}^{3} n_{2} r_{1}+3 n_{1}^{3} n_{2} r_{2}+\dot{\gamma} \theta_{0}\right)\right.$

$\left(-72 n_{1}^{2} n_{2}^{2}+144 n_{1}^{4} n_{2}^{2}-72 n_{1}^{6} n_{2}^{2}+144 n_{1}^{2} n_{2}^{4}-144 n_{1}^{4} n_{2}^{4}-72 n_{1}^{2} n_{2}^{6}+72 n_{1}-108 n_{1}^{2} r_{1}+36 n_{1}^{4} n_{1}-\right.$

$108 n_{2}^{2} r_{1}+288 n_{1}^{2} n_{2}^{2} r_{1}-648 n_{1}^{4} n_{2}^{2} r_{1}+432 n_{1}^{6} n_{2}^{2} r_{1}+36 n_{2}^{4} r_{1}-648 n_{1}^{2} n_{2}^{4} r_{1}+864 n_{1}^{4} n_{2}^{4} r_{1}+$

$432 n_{1}^{2} n_{2}^{6} r_{1}+144 n_{1}^{2} r_{1}^{2}-144 n_{1}^{4} r_{1}^{2}+144 n_{2}^{2} r_{1}^{2}-720 n_{1}^{2} n_{2}^{2} r_{1}^{2}+1080 n_{1}^{4} n_{2}^{2} r_{1}^{2}-648 n_{1}^{6} n_{2}^{2} r_{1}^{2}-144 n_{2}^{4} r_{1}^{2}+$

$1080 n_{1}^{2} n_{2}^{4} r_{1}^{2}-1296 n_{1}^{4} n_{2}^{4} r_{1}^{2}-648 n_{1}^{2} n_{2}^{6} r_{1}^{2}+288 n_{1}^{2} n_{2}^{2} r_{1}^{3}-576 n_{1}^{4} n_{2}^{2} r_{1}^{3}+288 n_{1}^{6} n_{2}^{2} r_{1}^{3}-576 n_{1}^{2} n_{2}^{4} r_{1}^{3}+$

$576 n_{1}^{4} n_{2}^{4} r_{1}^{3}+288 n_{1}^{2} n_{2}^{6} r_{1}^{3}+72 n_{1}^{2} n_{2}^{2} r_{2}+144 n_{1}^{4} n_{2}^{2} r_{2}-216 n_{1}^{6} n_{2}^{2} r_{2}+144 n_{1}^{2} n_{2}^{4} r_{2}-432 n_{1}^{4} n_{2}^{4} r_{2}-$

$216 n_{1}^{2} n_{2}^{6} r_{2}-36 n_{1}^{2} r_{1} r_{2}+108 n_{1}^{4} r_{1} r_{2}-36 n_{2}^{2} r_{1} r_{2}+288 n_{1}^{2} n_{2}^{2} r_{1} r_{2}-504 n_{1}^{4} n_{2}^{2} r_{1} r_{2}+432 n_{1}^{6} n_{2}^{2} r_{1} r_{2}+$

$108 n_{2}^{4} r_{1} r_{2}-504 n_{1}^{2} n_{2}^{4} r_{1} r_{2}+864 n_{1}^{4} n_{2}^{4} r_{1} r_{2}+432 n_{1}^{2} n_{2}^{6} r_{1} r_{2}-144 n_{1}^{2} n_{2}^{2} r_{1}^{2} r_{2}+360 n_{1}^{4} n_{2}^{2} r_{1}^{2} r_{2}-$

$216 n_{1}^{6} n_{2}^{2} r_{1}^{2} r_{2}+360 n_{1}^{2} n_{2}^{4} r_{1}^{2} r_{2}-432 n_{1}^{4} n_{2}^{4} r_{1}^{2} r_{2}-216 n_{1}^{2} n_{2}^{6} r_{1}^{2} r_{2}+72 \dot{\gamma}^{2} \theta_{0}^{2}-36 \dot{\gamma}^{2} n_{1}^{2} \theta_{0}^{2}+18 \dot{\gamma}^{2} n_{1}^{4} \theta_{0}^{2}-$

$36 \dot{\gamma}^{2} n_{2}^{2} \theta_{0}^{2}+36 \dot{\gamma}^{2} n_{1}^{2} n_{2}^{2} \theta_{0}^{2}+18 \dot{\gamma}^{2} n_{2}^{4} \theta_{0}^{2}+72 \dot{\gamma}^{2} n_{1}^{2} r_{1} \theta_{0}^{2}-72 \dot{\gamma}^{2} n_{1}^{4} r_{1} \theta_{0}^{2}+72 \dot{\gamma}^{2} n_{2}^{2} r_{1} \theta_{0}^{2}-$

$144 \dot{\gamma}^{2} n_{1}^{2} n_{2}^{2} r_{1} \theta_{0}^{2}-72 \dot{\gamma}^{2} n_{2}^{4} r_{1} \theta_{0}^{2}-36 \dot{\gamma}^{2} n_{1}^{2} r_{2} \theta_{0}^{2}+54 \dot{\gamma}^{2} n_{1}^{4} r_{2} \theta_{0}^{2}-36 \dot{\gamma}^{2} n_{2}^{2} r_{2} \theta_{0}^{2}+108 \dot{\gamma}^{2} n_{1}^{2} n_{2}^{2} r_{2} \theta_{0}^{2}+$

$\left.\left.54 \dot{\gamma}^{2} n_{2}^{4} r_{2} \theta_{0}^{2}\right)\right)+$ 
(2 (-36 $\dot{\gamma} \eta_{0}-36 \dot{\gamma} \hat{a} \hat{l} \eta_{0}+54 \dot{\gamma} n_{1}^{2} \eta_{0}+54 \dot{\gamma} \hat{a} n_{1}^{2} \eta_{0}-18 \dot{\gamma} n_{1}^{4} \eta_{0}-18 \dot{\gamma} a \hat{l} n_{1}^{4} \eta_{0}+54 \dot{\gamma} n_{2}^{2} \eta_{0}+54 \dot{\gamma} \hat{a} n_{2}^{2} \eta_{0}-$ $36 \dot{\gamma} n_{1}^{2} n_{2}^{2} \eta_{0}-72 \dot{\gamma} a \hat{l} n_{1}^{2} n_{2}^{2} \eta_{0}-24 \dot{\gamma} \hat{\beta} n_{1}^{2} n_{2}^{2} \eta_{0}+72 \dot{\gamma} n_{1}^{4} n_{2}^{2} \eta_{0}+144 \dot{\gamma} a \hat{l} n_{1}^{4} n_{2}^{2} \eta_{0}-24 \dot{\gamma} \hat{\beta} n_{1}^{4} n_{2}^{2} \eta_{0}-$ $72 \dot{\gamma} n_{1}^{6} n_{2}^{2} \eta_{0}-108 \dot{\gamma} \hat{a} 1 n_{1}^{6} n_{2}^{2} \eta_{0}+48 \dot{\gamma} \hat{\beta} n_{1}^{6} n_{2}^{2} \eta_{0}-18 \dot{\gamma} n_{2}^{4} \eta_{0}-18 \dot{\gamma} \hat{a} n_{2}^{4} \eta_{0}+72 \dot{\gamma} n_{1}^{2} n_{2}^{4} \eta_{0}+144 \dot{\gamma} a \hat{l} n_{1}^{2} n_{2}^{4} \eta_{0}-$ $24 \dot{\gamma} \hat{\beta} n_{1}^{2} n_{2}^{4} \eta_{0}-144 \dot{\gamma} n_{1}^{4} n_{2}^{4} \eta_{0}-216 \dot{\gamma} \hat{a} 1 n_{1}^{4} n_{2}^{4} \eta_{0}+96 \dot{\gamma} \hat{\beta} n_{1}^{4} n_{2}^{4} \eta_{0}-72 \dot{\gamma} n_{1}^{2} n_{2}^{6} \eta_{0}-108 \dot{\gamma} \hat{a} n_{1}^{2} n_{2}^{6} \eta_{0}+$ $48 \dot{\gamma} \hat{\beta} n_{1}^{2} n_{2}^{6} \eta_{0}-72 \dot{\gamma} n_{1}^{2} r_{1} \eta_{0}-72 \dot{\gamma} \hat{a l} n_{1}^{2} r_{1} \eta_{0}+72 \dot{\gamma} n_{1}^{4} n_{1} \eta_{0}+72 \dot{\gamma} \hat{a} n_{1}^{4} n_{1} \eta_{0}-72 \dot{\gamma} n_{2}^{2} r_{1} \eta_{0}-$ $72 \dot{\gamma}$ all $n_{2}^{2} r_{1} \eta_{0}+288 \dot{\gamma} n_{1}^{2} n_{2}^{2} r_{1} \eta_{0}+360 \dot{\gamma}$ al $n_{1}^{2} n_{2}^{2} r_{1} \eta_{0}-48 \dot{\gamma} \hat{\beta} n_{1}^{2} n_{2}^{2} r_{1} \eta_{0}-288 \dot{\gamma} n_{1}^{4} n_{2}^{2} r_{1} \eta_{0}-$ $468 \dot{\gamma} \hat{a} n_{1}^{4} n_{2}^{2} n_{1} \eta_{0}+72 \dot{\gamma} \hat{\beta} n_{1}^{4} n_{2}^{2} r_{1} \eta_{0}+144 \dot{\gamma} n_{1}^{6} n_{2}^{2} r_{1} \eta_{0}+252 \dot{\gamma} a \hat{l} n_{1}^{6} n_{2}^{2} n_{1} \eta_{0}-96 \dot{\gamma} \hat{\beta} n_{1}^{6} n_{2}^{2} r_{1} \eta_{0}+$ $72 \dot{\gamma} n_{2}^{4} r_{1} \eta_{0}+72 \dot{\gamma} a \hat{l} n_{2}^{4} r_{1} \eta_{0}-288 \dot{\gamma} n_{1}^{2} n_{2}^{4} r_{1} \eta_{0}-468 \dot{\gamma} a \hat{l} n_{1}^{2} n_{2}^{4} r_{1} \eta_{0}+72 \dot{\gamma} \hat{\beta} n_{1}^{2} n_{2}^{4} r_{1} \eta_{0}+288 \dot{\gamma} n_{1}^{4} n_{2}^{4} r_{1} \eta_{0}+$ $504 \dot{\gamma} a \hat{l} n_{1}^{4} n_{2}^{4} r_{1} \eta_{0}-192 \dot{\gamma} \hat{\beta} n_{1}^{4} n_{2}^{4} n_{1} \eta_{0}+144 \dot{\gamma} n_{1}^{2} n_{2}^{6} r_{1} \eta_{0}+252 \dot{\gamma}$ al $n_{1}^{2} n_{2}^{6} r_{1} \eta_{0}-96 \dot{\gamma} \hat{\beta} n_{1}^{2} n_{2}^{6} n_{1} \eta_{0}-$ $144 \dot{\gamma} n_{1}^{2} n_{2}^{2} r_{1}^{2} \eta_{0}-144 \dot{\gamma} \hat{a l} n_{1}^{2} n_{2}^{2} r_{1}^{2} \eta_{0}+216 \dot{\gamma} n_{1}^{4} n_{2}^{2} r_{1}^{2} \eta_{0}+288 \dot{\gamma}$ all $n_{1}^{4} n_{2}^{2} r_{1}^{2} \eta_{0}-48 \dot{\gamma} \hat{\beta} n_{1}^{4} n_{2}^{2} r_{1}^{2} \eta_{0}-$ $72 \dot{\gamma} n_{1}^{6} n_{2}^{2} r_{1}^{2} \eta_{0}-144 \dot{\gamma} a \hat{l} n_{1}^{6} n_{2}^{2} r_{1}^{2} \eta_{0}+48 \dot{\gamma} \hat{\beta} n_{1}^{6} n_{2}^{2} r_{1}^{2} \eta_{0}+216 \dot{\gamma} n_{1}^{2} n_{2}^{4} r_{1}^{2} \eta_{0}+288 \dot{\gamma} a \hat{l} n_{1}^{2} n_{2}^{4} r_{1}^{2} \eta_{0}-$ $48 \dot{\gamma} \hat{\beta} n_{1}^{2} n_{2}^{4} r_{1}^{2} \eta_{0}-144 \dot{\gamma} n_{1}^{4} n_{2}^{4} r_{1}^{2} \eta_{0}-288 \dot{\gamma} \hat{a} n_{1}^{4} n_{2}^{4} r_{1}^{2} \eta_{0}+96 \dot{\gamma} \hat{\beta} n_{1}^{4} n_{2}^{4} r_{1}^{2} \eta_{0}-72 \dot{\gamma} n_{1}^{2} n_{2}^{6} r_{1}^{2} \eta_{0}-$ $144 \dot{\gamma} \hat{a l} n_{1}^{2} n_{2}^{6} r_{1}^{2} \eta_{0}+48 \dot{\gamma} \hat{\beta} n_{1}^{2} n_{2}^{6} r_{1}^{2} \eta_{0}+18 \dot{\gamma} n_{1}^{2} r_{2} \eta_{0}+18 \dot{\gamma}$ al $n_{1}^{2} r_{2} \eta_{0}-54 \dot{\gamma} n_{1}^{4} r_{2} \eta_{0}-54 \dot{\gamma} \hat{a l} n_{1}^{4} r_{2} \eta_{0}+$ $18 \dot{\gamma} n_{2}^{2} r_{2} \eta_{0}+18 \dot{\gamma} \hat{a l} n_{2}^{2} r_{2} \eta_{0}-72 \dot{\gamma} n_{1}^{2} n_{2}^{2} r_{2} \eta_{0}-72 \dot{\gamma}$ al $n_{1}^{2} n_{2}^{2} r_{2} \eta_{0}+108 \dot{\gamma} n_{1}^{4} n_{2}^{2} r_{2} \eta_{0}+144 \dot{\gamma} a \hat{l} n_{1}^{4} n_{2}^{2} r_{2} \eta_{0}-$ $36 \dot{\gamma} \hat{a} 1 n_{1}^{6} n_{2}^{2} r_{2} \eta_{0}-54 \dot{\gamma} n_{2}^{4} r_{2} \eta_{0}-54 \dot{\gamma}$ al $n_{2}^{4} r_{2} \eta_{0}+108 \dot{\gamma} n_{1}^{2} n_{2}^{4} r_{2} \eta_{0}+144 \dot{\gamma}$ al $n_{1}^{2} n_{2}^{4} r_{2} \eta_{0}-$ $72 \dot{\gamma} \hat{a} n_{1}^{4} n_{2}^{4} r_{2} \eta_{0}-36 \dot{\gamma}$ âl $n_{1}^{2} n_{2}^{6} r_{2} \eta_{0}+72 \dot{\gamma} n_{1}^{2} n_{2}^{2} r_{1} r_{2} \eta_{0}+72 \dot{\gamma} \hat{a l l} n_{1}^{2} n_{2}^{2} r_{1} r_{2} \eta_{0}-108 \dot{\gamma} n_{1}^{4} n_{2}^{2} r_{1} r_{2} \eta_{0}-$ $108 \dot{\gamma} a 1 n_{1}^{4} n_{2}^{2} r_{1} r_{2} \eta_{0}+36 \dot{\gamma} \hat{a l} n_{1}^{6} n_{2}^{2} r_{1} r_{2} \eta_{0}-108 \dot{\gamma} n_{1}^{2} n_{2}^{4} r_{1} r_{2} \eta_{0}-108 \dot{\gamma} a 1 n_{1}^{2} n_{2}^{4} r_{1} r_{2} \eta_{0}+$ $72 \dot{\gamma} \hat{a} n_{1}^{4} n_{2}^{4} r_{1} r_{2} \eta_{0}+36 \dot{\gamma} \hat{a} 1 n_{1}^{2} n_{2}^{6} r_{1} r_{2} \eta_{0}-18 \dot{\gamma}^{2} n_{1}^{3} n_{2} \eta_{0} \theta_{0}+12 \dot{\gamma} \hat{\beta} n_{1}^{3} n_{2} \eta_{0} \theta_{0}-36 \dot{\gamma}^{2} n_{1}^{5} n_{2} \eta_{0} \theta_{0}-$ $54 \dot{\gamma}^{2} \hat{a} 1 n_{1}^{5} n_{2} \eta_{0} \theta_{0}+24 \dot{\gamma}^{2} \hat{\beta} n_{1}^{5} n_{2} \eta_{0} \theta_{0}+18 \dot{\gamma}^{2} n_{1} n_{2}^{3} \eta_{0} \theta_{0}-12 \dot{\gamma}^{2} \hat{\beta} n_{1} n_{2}^{3} \eta_{0} \theta_{0}+36 \dot{\gamma}^{2} n_{1} n_{2}^{5} \eta_{0} \theta_{0}+$ $54 \dot{\gamma}^{2} \hat{a} n_{1} n_{2}^{5} \eta_{0} \theta_{0}-24 \dot{\gamma}^{2} \hat{\beta} n_{1} n_{2}^{5} \eta_{0} \theta_{0}-36 \dot{\gamma}^{2} n_{1}^{3} n_{2} r_{1} \eta_{0} \theta_{0}-72 \dot{\gamma}^{2} \hat{a} n_{1}^{3} n_{2} r_{1} \eta_{0} \theta_{0}+24 \dot{\gamma}^{2} \hat{\beta} n_{1}^{3} n_{2} r_{1} \eta_{0} \theta_{0}+$ $36 \dot{\gamma}^{2} n_{1}^{5} n_{2} r_{1} \eta_{0} \theta_{0}+72 \dot{\gamma}^{2} \hat{a} 1 n_{1}^{5} n_{2} r_{1} \eta_{0} \theta_{0}-24 \dot{\gamma}^{2} \hat{\beta} n_{1}^{5} n_{2} r_{1} \eta_{0} \theta_{0}+36 \dot{\gamma}^{2} n_{1} n_{2}^{3} r_{1} \eta_{0} \theta_{0}+72 \dot{\gamma}^{2} \hat{a} 1 n_{1} n_{2}^{3} r_{1} \eta_{0} \theta_{0}-$ $24 \dot{\gamma}^{2} \hat{\beta} n_{1} n_{2}^{3} r_{1} \eta_{0} \theta_{0}-36 \dot{\gamma}^{2} n_{1} n_{2}^{5} r_{1} \eta_{0} \theta_{0}-72 \dot{\gamma}^{2} \hat{a} n_{1} n_{2}^{5} r_{1} \eta_{0} \theta_{0}+24 \dot{\gamma}^{2} \hat{\beta} n_{1} n_{2}^{5} r_{1} \eta_{0} \theta_{0}-$ $18 \dot{\gamma}^{2}$ all $\left.n_{1}^{5} n_{2} r_{2} \eta_{0} \theta_{0}+18 \dot{\gamma}^{2} \hat{a l} n_{1} n_{2}^{5} r_{2} \eta_{0} \theta_{0}\right)$ $\left(-12 r_{1}+18 n_{1}^{2} r_{1}-6 n_{1}^{4} r_{1}+12 n_{1}^{2} n_{2}^{2} r_{1}-24 n_{1}^{2} r_{1}^{2}+24 n_{1}^{4} r_{1}^{2}+24 n_{1}^{2} n_{2}^{2} r_{1}^{2}-24 n_{1}^{2} n_{2}^{2} r_{2}+6 n_{1}^{2} r_{1} r_{2}-\right.$ $\left.\left.18 n_{1}^{4} r_{1} r_{2}-12 n_{1}^{2} n_{2}^{2} r_{1} r_{2}+6 \dot{\gamma} n_{1} n_{2} \theta_{0}-6 \dot{\gamma} n_{1} n_{2} r_{2} \theta_{0}-6 \dot{\gamma}^{2} \theta_{0}^{2}\right)\right) /$

$\left(\left(12 n_{1} n_{2}-18 n_{1}^{3} n_{2}+6 n_{1}^{5} n_{2}-12 n_{1} n_{2}^{3}+6 n_{1}^{3} n_{2}^{3}-12 n_{1} n_{2} r_{1}+42 n_{1}^{3} n_{2} r_{1}-30 n_{1}^{5} n_{2} r_{1}+30 n_{1} n_{2}^{3} r_{1}-\right.\right.$ $30 n_{1}^{3} n_{2}^{3} r_{1}-24 n_{1}^{3} n_{2} r_{1}^{2}+24 n_{1}^{5} n_{2} r_{1}^{2}+24 n_{1}^{3} n_{2}^{3} r_{1}^{2}-6 n_{1}^{3} n_{2} r_{2}+18 n_{1}^{5} n_{2} r_{2}-12 n_{1} n_{2}^{3} r_{2}+18 n_{1}^{3} n_{2}^{3} r_{2}+$ $6 n_{1}^{3} n_{2} r_{1} r_{2}-18 n_{1}^{5} n_{2} r_{1} r_{2}-6 n_{1} n_{2}^{3} r_{1} r_{2}-18 n_{1}^{3} n_{2}^{3} r_{1} r_{2}+6 \dot{\gamma} \theta_{0}-9 \dot{\gamma} n_{1}^{2} \theta_{0}+3 \dot{\gamma} n_{1}^{4} \theta_{0}+3 \dot{\gamma} n_{2}^{2} \theta_{0}+$ $\left.3 \dot{\gamma} n_{1}^{2} n_{2}^{2} \theta_{0}+12 \dot{\gamma} n_{1}^{2} r_{1} \theta_{0}-12 \dot{\gamma} n_{1}^{4} r_{1} \theta_{0}-12 \dot{\gamma} n_{1}^{2} n_{2}^{2} r_{1} \theta_{0}-3 \dot{\gamma} n_{1}^{2} r_{2} \theta_{0}+9 \dot{\gamma} n_{1}^{4} r_{2} \theta_{0}-3 \dot{\gamma} n_{2}^{2} r_{2} \theta_{0}+9 \dot{\gamma} n_{1}^{2} n_{2}^{2} r_{2} \theta_{0}\right)$ $\left(-72 n_{1}^{2} n_{2}^{2}+144 n_{1}^{4} n_{2}^{2}-72 n_{1}^{6} n_{2}^{2}+144 n_{1}^{2} n_{2}^{4}-144 n_{1}^{4} n_{2}^{4}-72 n_{1}^{2} n_{2}^{6}+72 n_{1}-108 n_{1}^{2} r_{1}+36 n_{1}^{4} n_{1}-108 n_{2}^{2} n_{1}+\right.$ $288 n_{1}^{2} n_{2}^{2} r_{1}-648 n_{1}^{4} n_{2}^{2} r_{1}+432 n_{1}^{6} n_{2}^{2} r_{1}+36 n_{2}^{4} r_{1}-648 n_{1}^{2} n_{2}^{4} r_{1}+864 n_{1}^{4} n_{2}^{4} r_{1}+432 n_{1}^{2} n_{2}^{6} r_{1}+144 n_{1}^{2} r_{1}^{2}-$ $144 n_{1}^{4} r_{1}^{2}+144 n_{2}^{2} r_{1}^{2}-720 n_{1}^{2} n_{2}^{2} r_{1}^{2}+1080 n_{1}^{4} n_{2}^{2} r_{1}^{2}-648 n_{1}^{6} n_{2}^{2} r_{1}^{2}-144 n_{2}^{4} r_{1}^{2}+1080 n_{1}^{2} n_{2}^{4} r_{1}^{2}-1296 n_{1}^{4} n_{2}^{4} r_{1}^{2}-$ $648 n_{1}^{2} n_{2}^{6} r_{1}^{2}+288 n_{1}^{2} n_{2}^{2} r_{1}^{3}-576 n_{1}^{4} n_{2}^{2} r_{1}^{3}+288 n_{1}^{6} n_{2}^{2} r_{1}^{3}-576 n_{1}^{2} n_{2}^{4} r_{1}^{3}+576 n_{1}^{4} n_{2}^{4} r_{1}^{3}+288 n_{1}^{2} n_{2}^{6} r_{1}^{3}+$ $72 n_{1}^{2} n_{2}^{2} r_{2}+144 n_{1}^{4} n_{2}^{2} r_{2}-216 n_{1}^{6} n_{2}^{2} r_{2}+144 n_{1}^{2} n_{2}^{4} r_{2}-432 n_{1}^{4} n_{2}^{4} r_{2}-216 n_{1}^{2} n_{2}^{6} r_{2}-36 n_{1}^{2} r_{1} r_{2}+$ $108 n_{1}^{4} r_{1} r_{2}-36 n_{2}^{2} r_{1} r_{2}+288 n_{1}^{2} n_{2}^{2} r_{1} r_{2}-504 n_{1}^{4} n_{2}^{2} r_{1} r_{2}+432 n_{1}^{6} n_{2}^{2} r_{1} r_{2}+108 n_{2}^{4} r_{1} r_{2}-504 n_{1}^{2} n_{2}^{4} r_{1} r_{2}+$ $864 n_{1}^{4} n_{2}^{4} r_{1} r_{2}+432 n_{1}^{2} n_{2}^{6} r_{1} r_{2}-144 n_{1}^{2} n_{2}^{2} r_{1}^{2} r_{2}+360 n_{1}^{4} n_{2}^{2} r_{1}^{2} r_{2}-216 n_{1}^{6} n_{2}^{2} r_{1}^{2} r_{2}+360 n_{1}^{2} n_{2}^{4} r_{1}^{2} r_{2}-$ $432 n_{1}^{4} n_{2}^{4} r_{1}^{2} r_{2}-216 n_{1}^{2} n_{2}^{6} r_{1}^{2} r_{2}+72 \dot{\gamma}^{2} \theta_{0}^{2}-36 \dot{\gamma}^{2} n_{1}^{2} \theta_{0}^{2}+18 \dot{\gamma}^{2} n_{1}^{4} \theta_{0}^{2}-36 \dot{\gamma}^{2} n_{2}^{2} \theta_{0}^{2}+36 \dot{\gamma}^{2} n_{1}^{2} n_{2}^{2} \theta_{0}^{2}+$ $18 \dot{\gamma}^{2} n_{2}^{4} \theta_{0}^{2}+72 \dot{\gamma}^{2} n_{1}^{2} r_{1} \theta_{0}^{2}-72 \dot{\gamma}^{2} n_{1}^{4} r_{1} \theta_{0}^{2}+72 \dot{\gamma}^{2} n_{2}^{2} r_{1} \theta_{0}^{2}-144 \dot{\gamma}^{2} n_{1}^{2} n_{2}^{2} r_{1} \theta_{0}^{2}-72 \dot{\gamma}^{2} n_{2}^{4} r_{1} \theta_{0}^{2}-$ $\left.\left.36 \dot{\gamma}^{2} n_{1}^{2} r_{2} \theta_{0}^{2}+54 \dot{\gamma}^{2} n_{1}^{4} r_{2} \theta_{0}^{2}-36 \dot{\gamma}^{2} n_{2}^{2} r_{2} \theta_{0}^{2}+108 \dot{\gamma}^{2} n_{1}^{2} n_{2}^{2} r_{2} \theta_{0}^{2}+54 \dot{\gamma}^{2} n_{2}^{4} r_{2} \theta_{0}^{2}\right)\right)+$

$\left(\left(-2 n_{1} n_{2}+n_{1} n_{2}^{3}+2 n_{1} n_{2} r_{1}-4 n_{1} n_{2}^{3} r_{1}+3 n_{1} n_{2}^{3} r_{2}-\dot{\gamma} \theta_{0}\right)\right.$

$\left(\left(6 \dot{\gamma} \eta_{0}+6 \dot{\gamma} \hat{a} \mathbf{l} \eta_{0}-9 \dot{\gamma} n_{1}^{2} \eta_{0}-9 \dot{\gamma} a \hat{l} n_{1}^{2} \eta_{0}+3 \dot{\gamma} n_{1}^{4} \eta_{0}+3 \dot{\gamma} \hat{a} 1 n_{1}^{4} \eta_{0}-12 \dot{\gamma} n_{1}^{2} n_{2}^{2} \eta_{0}-12 \dot{\gamma} \hat{a l} n_{1}^{2} n_{2}^{2} \eta_{0}+\right.\right.$ $8 \dot{\gamma} \hat{\beta} n_{1}^{2} n_{2}^{2} \eta_{0}+6 \dot{\gamma} n_{1}^{4} n_{2}^{2} \eta_{0}+6 \dot{\gamma} \hat{a} \hat{1} n_{1}^{4} n_{2}^{2} \eta_{0}-4 \dot{\gamma} \hat{\beta} n_{1}^{4} n_{2}^{2} \eta_{0}+12 \dot{\gamma} n_{1}^{2} r_{1} \eta_{0}+12 \dot{\gamma} \hat{a} n_{1}^{2} r_{1} \eta_{0}-12 \dot{\gamma} n_{1}^{4} r_{1} \eta_{0}-$ $12 \dot{\gamma} \hat{a} n_{1}^{4} n_{1} \eta_{0}-6 \dot{\gamma} n_{1}^{2} n_{2}^{2} n_{1} \eta_{0}-12 \dot{\gamma} a \hat{l} n_{1}^{2} n_{2}^{2} r_{1} \eta_{0}+4 \dot{\gamma} \hat{\beta} n_{1}^{2} n_{2}^{2} r_{1} \eta_{0}-6 \dot{\gamma} n_{1}^{4} n_{2}^{2} n_{1} \eta_{0}+4 \dot{\gamma} \hat{\beta} n_{1}^{4} n_{2}^{2} r_{1} \eta_{0}-$ $3 \dot{\gamma} n_{1}^{2} r_{2} \eta_{0}-3 \dot{\gamma} a 1 n_{1}^{2} r_{2} \eta_{0}+9 \dot{\gamma} n_{1}^{4} r_{2} \eta_{0}+9 \dot{\gamma} a \hat{l} n_{1}^{4} r_{2} \eta_{0}-6 \dot{\gamma} a \hat{l} n_{1}^{4} n_{2}^{2} r_{2} \eta_{0}-3 \dot{\gamma}^{2} n_{1} n_{2} \eta_{0} \theta_{0}-$ $\left.6 \dot{\gamma}^{2} \hat{a} n_{1} n_{2} \eta_{0} \theta_{0}+2 \dot{\gamma}^{2} \hat{\beta} n_{1} n_{2} \eta_{0} \theta_{0}+9 \dot{\gamma}^{2} n_{1}^{3} n_{2} \eta_{0} \theta_{0}+12 \dot{\gamma}^{2} \hat{a} n_{1}^{3} n_{2} \eta_{0} \theta_{0}-6 \dot{\gamma}^{2} \hat{\beta} n_{1}^{3} n_{2} \eta_{0} \theta_{0}\right) /$ 
$\left(12 n_{1} n_{2}-18 n_{1}^{3} n_{2}+6 n_{1}^{5} n_{2}-12 n_{1} n_{2}^{3}+6 n_{1}^{3} n_{2}^{3}-12 n_{1} n_{2} r_{1}+42 n_{1}^{3} n_{2} r_{1}-30 n_{1}^{5} n_{2} r_{1}+30 n_{1} n_{2}^{3} r_{1}-30 n_{1}^{3} n_{2}^{3} r_{1}-\right.$ $24 n_{1}^{3} n_{2} r_{1}^{2}+24 n_{1}^{5} n_{2} r_{1}^{2}+24 n_{1}^{3} n_{2}^{3} r_{1}^{2}-6 n_{1}^{3} n_{2} r_{2}+18 n_{1}^{5} n_{2} r_{2}-12 n_{1} n_{2}^{3} r_{2}+18 n_{1}^{3} n_{2}^{3} r_{2}+6 n_{1}^{3} n_{2} r_{1} r_{2}-$ $18 n_{1}^{5} n_{2} r_{1} r_{2}-6 n_{1} n_{2}^{3} r_{1} r_{2}-18 n_{1}^{3} n_{2}^{3} r_{1} r_{2}+6 \dot{\gamma} \theta_{0}-9 \dot{\gamma} n_{1}^{2} \theta_{0}+3 \dot{\gamma} n_{1}^{4} \theta_{0}+3 \dot{\gamma} n_{2}^{2} \theta_{0}+3 \dot{\gamma} n_{1}^{2} n_{2}^{2} \theta_{0}+$ $\left.12 \dot{\gamma} n_{1}^{2} r_{1} \theta_{0}-12 \dot{\gamma} n_{1}^{4} r_{1} \theta_{0}-12 \dot{\gamma} n_{1}^{2} n_{2}^{2} r_{1} \theta_{0}-3 \dot{\gamma} n_{1}^{2} r_{2} \theta_{0}+9 \dot{\gamma} n_{1}^{4} r_{2} \theta_{0}-3 \dot{\gamma} n_{2}^{2} r_{2} \theta_{0}+9 \dot{\gamma} n_{1}^{2} n_{2}^{2} r_{2} \theta_{0}\right)-$ $\left(\left(-36 \dot{\gamma} \eta_{0}-36 \dot{\gamma}\right.\right.$ al $\eta_{0}+54 \dot{\gamma} n_{1}^{2} \eta_{0}+54 \dot{\gamma}$ al $n_{1}^{2} \eta_{0}-18 \dot{\gamma} n_{1}^{4} \eta_{0}-18 \dot{\gamma} \hat{a l} n_{1}^{4} \eta_{0}+54 \dot{\gamma} n_{2}^{2} \eta_{0}+54 \dot{\gamma} \hat{a l} n_{2}^{2} \eta_{0}-$ $36 \dot{\gamma} n_{1}^{2} n_{2}^{2} \eta_{0}-72 \dot{\gamma} \hat{a} n_{1}^{2} n_{2}^{2} \eta_{0}-24 \dot{\gamma} \hat{\beta} n_{1}^{2} n_{2}^{2} \eta_{0}+72 \dot{\gamma} n_{1}^{4} n_{2}^{2} \eta_{0}+144 \dot{\gamma} a \hat{l} n_{1}^{4} n_{2}^{2} \eta_{0}-24 \dot{\gamma} \hat{\beta} n_{1}^{4} n_{2}^{2} \eta_{0}-$ $72 \dot{\gamma} n_{1}^{6} n_{2}^{2} \eta_{0}-108 \dot{\gamma} \hat{a} 1 n_{1}^{6} n_{2}^{2} \eta_{0}+48 \dot{\gamma} \hat{\beta} n_{1}^{6} n_{2}^{2} \eta_{0}-18 \dot{\gamma} n_{2}^{4} \eta_{0}-18 \dot{\gamma} \hat{a} n_{2}^{4} \eta_{0}+72 \dot{\gamma} n_{1}^{2} n_{2}^{4} \eta_{0}+$ $144 \dot{\gamma} \hat{a} 1 n_{1}^{2} n_{2}^{4} \eta_{0}-24 \dot{\gamma} \hat{\beta} n_{1}^{2} n_{2}^{4} \eta_{0}-144 \dot{\gamma} n_{1}^{4} n_{2}^{4} \eta_{0}-216 \dot{\gamma} \hat{a} n_{1}^{4} n_{2}^{4} \eta_{0}+96 \dot{\gamma} \hat{\beta} n_{1}^{4} n_{2}^{4} \eta_{0}-72 \dot{\gamma} n_{1}^{2} n_{2}^{6} \eta_{0}-$ $108 \dot{\gamma} \hat{a} n_{1}^{2} n_{2}^{6} \eta_{0}+48 \dot{\gamma} \hat{\beta} n_{1}^{2} n_{2}^{6} \eta_{0}-72 \dot{\gamma} n_{1}^{2} r_{1} \eta_{0}-72 \dot{\gamma}$ al $n_{1}^{2} r_{1} \eta_{0}+72 \dot{\gamma} n_{1}^{4} r_{1} \eta_{0}+72 \dot{\gamma}$ âl $n_{1}^{4} r_{1} \eta_{0}-$ $72 \dot{\gamma} n_{2}^{2} r_{1} \eta_{0}-72 \dot{\gamma} \hat{a l} n_{2}^{2} r_{1} \eta_{0}+288 \dot{\gamma} n_{1}^{2} n_{2}^{2} r_{1} \eta_{0}+360 \dot{\gamma}$ al $n_{1}^{2} n_{2}^{2} r_{1} \eta_{0}-48 \dot{\gamma} \hat{\beta} n_{1}^{2} n_{2}^{2} r_{1} \eta_{0}-$ $288 \dot{\gamma} n_{1}^{4} n_{2}^{2} r_{1} \eta_{0}-468 \dot{\gamma} \hat{a} n_{1}^{4} n_{2}^{2} r_{1} \eta_{0}+72 \dot{\gamma} \hat{\beta} n_{1}^{4} n_{2}^{2} r_{1} \eta_{0}+144 \dot{\gamma} n_{1}^{6} n_{2}^{2} r_{1} \eta_{0}+252 \dot{\gamma}$ all $n_{1}^{6} n_{2}^{2} r_{1} \eta_{0}-$ $96 \dot{\gamma} \hat{\beta} n_{1}^{6} n_{2}^{2} r_{1} \eta_{0}+72 \dot{\gamma} n_{2}^{4} r_{1} \eta_{0}+72 \dot{\gamma}$ al $n_{2}^{4} r_{1} \eta_{0}-288 \dot{\gamma} n_{1}^{2} n_{2}^{4} r_{1} \eta_{0}-468 \dot{\gamma} \hat{a} n_{1}^{2} n_{2}^{4} r_{1} \eta_{0}+$ $72 \dot{\gamma} \hat{\beta} n_{1}^{2} n_{2}^{4} r_{1} \eta_{0}+288 \dot{\gamma} n_{1}^{4} n_{2}^{4} r_{1} \eta_{0}+504 \dot{\gamma} a 1 n_{1}^{4} n_{2}^{4} r_{1} \eta_{0}-192 \dot{\gamma} \hat{\beta} n_{1}^{4} n_{2}^{4} r_{1} \eta_{0}+144 \dot{\gamma} n_{1}^{2} n_{2}^{6} r_{1} \eta_{0}+$ $252 \dot{\gamma} \hat{a} n_{1}^{2} n_{2}^{6} r_{1} \eta_{0}-96 \dot{\gamma} \hat{\beta} n_{1}^{2} n_{2}^{6} r_{1} \eta_{0}-144 \dot{\gamma} n_{1}^{2} n_{2}^{2} r_{1}^{2} \eta_{0}-144 \dot{\gamma}$ al $n_{1}^{2} n_{2}^{2} r_{1}^{2} \eta_{0}+216 \dot{\gamma} n_{1}^{4} n_{2}^{2} r_{1}^{2} \eta_{0}+$ $288 \dot{\gamma} \hat{a} 1 n_{1}^{4} n_{2}^{2} r_{1}^{2} \eta_{0}-48 \dot{\gamma} \hat{\beta} n_{1}^{4} n_{2}^{2} r_{1}^{2} \eta_{0}-72 \dot{\gamma} n_{1}^{6} n_{2}^{2} r_{1}^{2} \eta_{0}-144 \dot{\gamma} a \hat{l} n_{1}^{6} n_{2}^{2} r_{1}^{2} \eta_{0}+48 \dot{\gamma} \hat{\beta} n_{1}^{6} n_{2}^{2} r_{1}^{2} \eta_{0}+$ $216 \dot{\gamma} n_{1}^{2} n_{2}^{4} r_{1}^{2} \eta_{0}+288 \dot{\gamma} \hat{a} n_{1}^{2} n_{2}^{4} r_{1}^{2} \eta_{0}-48 \dot{\gamma} \hat{\beta} n_{1}^{2} n_{2}^{4} r_{1}^{2} \eta_{0}-144 \dot{\gamma} n_{1}^{4} n_{2}^{4} r_{1}^{2} \eta_{0}-288 \dot{\gamma}$ âl $n_{1}^{4} n_{2}^{4} r_{1}^{2} \eta_{0}+$ $96 \dot{\gamma} \hat{\beta} n_{1}^{4} n_{2}^{4} r_{1}^{2} \eta_{0}-72 \dot{\gamma} n_{1}^{2} n_{2}^{6} r_{1}^{2} \eta_{0}-144 \dot{\gamma} \hat{a} 1 n_{1}^{2} n_{2}^{6} r_{1}^{2} \eta_{0}+48 \dot{\gamma} \hat{\beta} n_{1}^{2} n_{2}^{6} r_{1}^{2} \eta_{0}+18 \dot{\gamma} n_{1}^{2} r_{2} \eta_{0}+$ $18 \dot{\gamma} \hat{a l} n_{1}^{2} r_{2} \eta_{0}-54 \dot{\gamma} n_{1}^{4} r_{2} \eta_{0}-54 \dot{\gamma}$ al $n_{1}^{4} r_{2} \eta_{0}+18 \dot{\gamma} n_{2}^{2} r_{2} \eta_{0}+18 \dot{\gamma}$ all $n_{2}^{2} r_{2} \eta_{0}-72 \dot{\gamma} n_{1}^{2} n_{2}^{2} r_{2} \eta_{0}-$ $72 \dot{\gamma} a \hat{a} n_{1}^{2} n_{2}^{2} r_{2} \eta_{0}+108 \dot{\gamma} n_{1}^{4} n_{2}^{2} r_{2} \eta_{0}+144 \dot{\gamma}$ all $n_{1}^{4} n_{2}^{2} r_{2} \eta_{0}-36 \dot{\gamma}$ all $n_{1}^{6} n_{2}^{2} r_{2} \eta_{0}-54 \dot{\gamma} n_{2}^{4} r_{2} \eta_{0}-$ $54 \dot{\gamma} \hat{a l} n_{2}^{4} r_{2} \eta_{0}+108 \dot{\gamma} n_{1}^{2} n_{2}^{4} r_{2} \eta_{0}+144 \dot{\gamma}$ âl $n_{1}^{2} n_{2}^{4} r_{2} \eta_{0}-72 \dot{\gamma} \hat{a} 1 n_{1}^{4} n_{2}^{4} r_{2} \eta_{0}-36 \dot{\gamma} a 1 n_{1}^{2} n_{2}^{6} r_{2} \eta_{0}+$ $72 \dot{\gamma} n_{1}^{2} n_{2}^{2} r_{1} r_{2} \eta_{0}+72 \dot{\gamma} \hat{a l} n_{1}^{2} n_{2}^{2} r_{1} r_{2} \eta_{0}-108 \dot{\gamma} n_{1}^{4} n_{2}^{2} r_{1} r_{2} \eta_{0}-108 \dot{\gamma} \hat{a l} n_{1}^{4} n_{2}^{2} r_{1} r_{2} \eta_{0}+$ $36 \dot{\gamma} \hat{a} 1 n_{1}^{6} n_{2}^{2} r_{1} r_{2} \eta_{0}-108 \dot{\gamma} n_{1}^{2} n_{2}^{4} r_{1} r_{2} \eta_{0}-108 \dot{\gamma} \hat{a l} n_{1}^{2} n_{2}^{4} r_{1} r_{2} \eta_{0}+72 \dot{\gamma} \hat{a l} n_{1}^{4} n_{2}^{4} r_{1} r_{2} \eta_{0}+$ $36 \dot{\gamma}$ al $n_{1}^{2} n_{2}^{6} r_{1} r_{2} \eta_{0}-18 \dot{\gamma}^{2} n_{1}^{3} n_{2} \eta_{0} \theta_{0}+12 \dot{\gamma}^{2} \hat{\beta} n_{1}^{3} n_{2} \eta_{0} \theta_{0}-36 \dot{\gamma}^{2} n_{1}^{5} n_{2} \eta_{0} \theta_{0}-54 \dot{\gamma}^{2} \hat{a} n_{1}^{5} n_{2} \eta_{0} \theta_{0}+$ $24 \dot{\gamma}^{2} \hat{\beta} n_{1}^{5} n_{2} \eta_{0} \theta_{0}+18 \dot{\gamma}^{2} n_{1} n_{2}^{3} \eta_{0} \theta_{0}-12 \dot{\gamma}^{2} \hat{\beta} n_{1} n_{2}^{3} \eta_{0} \theta_{0}+36 \dot{\gamma}^{2} n_{1} n_{2}^{5} \eta_{0} \theta_{0}+54 \dot{\gamma}^{2} \hat{a} 1 n_{1} n_{2}^{5} \eta_{0} \theta_{0}-$ $24 \dot{\gamma}^{2} \hat{\beta} n_{1} n_{2}^{5} \eta_{0} \theta_{0}-36 \dot{\gamma}^{2} n_{1}^{3} n_{2} r_{1} \eta_{0} \theta_{0}-72 \dot{\gamma}^{2} \hat{a} n_{1}^{3} n_{2} r_{1} \eta_{0} \theta_{0}+24 \dot{\gamma}^{2} \hat{\beta} n_{1}^{3} n_{2} r_{1} \eta_{0} \theta_{0}+$ $36 \dot{\gamma}^{2} n_{1}^{5} n_{2} r_{1} \eta_{0} \theta_{0}+72 \dot{\gamma}^{2} \hat{a} 1 n_{1}^{5} n_{2} r_{1} \eta_{0} \theta_{0}-24 \dot{\gamma}^{2} \hat{\beta} n_{1}^{5} n_{2} r_{1} \eta_{0} \theta_{0}+36 \dot{\gamma}^{2} n_{1} n_{2}^{3} r_{1} \eta_{0} \theta_{0}+$ $72 \dot{\gamma}^{2} \hat{a} n_{1} n_{2}^{3} r_{1} \eta_{0} \theta_{0}-24 \dot{\gamma}^{2} \hat{\beta} n_{1} n_{2}^{3} r_{1} \eta_{0} \theta_{0}-36 \dot{\gamma}^{2} n_{1} n_{2}^{5} r_{1} \eta_{0} \theta_{0}-72 \dot{\gamma}^{2} \hat{a} 1 n_{1} n_{2}^{5} r_{1} \eta_{0} \theta_{0}+$ $\left.24 \dot{\gamma}^{2} \hat{\beta} n_{1} n_{2}^{5} r_{1} \eta_{0} \theta_{0}-18 \dot{\gamma}^{2} \hat{a l} n_{1}^{5} n_{2} r_{2} \eta_{0} \theta_{0}+18 \dot{\gamma}^{2} \hat{a} 1 n_{1} n_{2}^{5} r_{2} \eta_{0} \theta_{0}\right)$

$\left(-12 r_{1}+18 n_{1}^{2} r_{1}-6 n_{1}^{4} r_{1}+12 n_{1}^{2} n_{2}^{2} r_{1}-24 n_{1}^{2} r_{1}^{2}+24 n_{1}^{4} r_{1}^{2}+24 n_{1}^{2} n_{2}^{2} r_{1}^{2}-24 n_{1}^{2} n_{2}^{2} r_{2}+6 n_{1}^{2} r_{1} r_{2}-\right.$ $\left.\left.18 n_{1}^{4} r_{1} r_{2}-12 n_{1}^{2} n_{2}^{2} r_{1} r_{2}+6 \dot{\gamma} n_{1} n_{2} \theta_{0}-6 \dot{\gamma} n_{1} n_{2} r_{2} \theta_{0}-6 \dot{\gamma}^{2} \theta_{0}^{2}\right)\right) /$

$\left(\left(12 n_{1} n_{2}-18 n_{1}^{3} n_{2}+6 n_{1}^{5} n_{2}-12 n_{1} n_{2}^{3}+6 n_{1}^{3} n_{2}^{3}-12 n_{1} n_{2} r_{1}+42 n_{1}^{3} n_{2} r_{1}-30 n_{1}^{5} n_{2} r_{1}+30 n_{1} n_{2}^{3} r_{1}-\right.\right.$ $30 n_{1}^{3} n_{2}^{3} r_{1}-24 n_{1}^{3} n_{2} r_{1}^{2}+24 n_{1}^{5} n_{2} r_{1}^{2}+24 n_{1}^{3} n_{2}^{3} r_{1}^{2}-6 n_{1}^{3} n_{2} r_{2}+18 n_{1}^{5} n_{2} r_{2}-12 n_{1} n_{2}^{3} r_{2}+18 n_{1}^{3} n_{2}^{3} r_{2}+$ $6 n_{1}^{3} n_{2} r_{1} r_{2}-18 n_{1}^{5} n_{2} r_{1} r_{2}-6 n_{1} n_{2}^{3} r_{1} r_{2}-18 n_{1}^{3} n_{2}^{3} r_{1} r_{2}+6 \dot{\gamma} \theta_{0}-9 \dot{\gamma} n_{1}^{2} \theta_{0}+3 \dot{\gamma} n_{1}^{4} \theta_{0}+3 \dot{\gamma} n_{2}^{2} \theta_{0}+$ $3 \dot{\gamma} n_{1}^{2} n_{2}^{2} \theta_{0}+12 \dot{\gamma} n_{1}^{2} r_{1} \theta_{0}-12 \dot{\gamma} n_{1}^{4} r_{1} \theta_{0}-12 \dot{\gamma} n_{1}^{2} n_{2}^{2} r_{1} \theta_{0}-3 \dot{\gamma} n_{1}^{2} r_{2} \theta_{0}+9 \dot{\gamma} n_{1}^{4} r_{2} \theta_{0}-3 \dot{\gamma} n_{2}^{2} r_{2} \theta_{0}+$ $\left.9 \dot{\gamma} n_{1}^{2} n_{2}^{2} r_{2} \theta_{0}\right)\left(-72 n_{1}^{2} n_{2}^{2}+144 n_{1}^{4} n_{2}^{2}-72 n_{1}^{6} n_{2}^{2}+144 n_{1}^{2} n_{2}^{4}-144 n_{1}^{4} n_{2}^{4}-72 n_{1}^{2} n_{2}^{6}+72 r_{1}-108 n_{1}^{2} r_{1}+\right.$ $36 n_{1}^{4} r_{1}-108 n_{2}^{2} r_{1}+288 n_{1}^{2} n_{2}^{2} r_{1}-648 n_{1}^{4} n_{2}^{2} r_{1}+432 n_{1}^{6} n_{2}^{2} r_{1}+36 n_{2}^{4} r_{1}-648 n_{1}^{2} n_{2}^{4} r_{1}+864 n_{1}^{4} n_{2}^{4} r_{1}+$ $432 n_{1}^{2} n_{2}^{6} r_{1}+144 n_{1}^{2} r_{1}^{2}-144 n_{1}^{4} r_{1}^{2}+144 n_{2}^{2} r_{1}^{2}-720 n_{1}^{2} n_{2}^{2} r_{1}^{2}+1080 n_{1}^{4} n_{2}^{2} r_{1}^{2}-648 n_{1}^{6} n_{2}^{2} r_{1}^{2}-144 n_{2}^{4} r_{1}^{2}+$ $1080 n_{1}^{2} n_{2}^{4} r_{1}^{2}-1296 n_{1}^{4} n_{2}^{4} r_{1}^{2}-648 n_{1}^{2} n_{2}^{6} r_{1}^{2}+288 n_{1}^{2} n_{2}^{2} r_{1}^{3}-576 n_{1}^{4} n_{2}^{2} r_{1}^{3}+288 n_{1}^{6} n_{2}^{2} r_{1}^{3}-576 n_{1}^{2} n_{2}^{4} r_{1}^{3}+$ $576 n_{1}^{4} n_{2}^{4} r_{1}^{3}+288 n_{1}^{2} n_{2}^{6} r_{1}^{3}+72 n_{1}^{2} n_{2}^{2} r_{2}+144 n_{1}^{4} n_{2}^{2} r_{2}-216 n_{1}^{6} n_{2}^{2} r_{2}+144 n_{1}^{2} n_{2}^{4} r_{2}-432 n_{1}^{4} n_{2}^{4} r_{2}-$ $216 n_{1}^{2} n_{2}^{6} r_{2}-36 n_{1}^{2} r_{1} r_{2}+108 n_{1}^{4} r_{1} r_{2}-36 n_{2}^{2} r_{1} r_{2}+288 n_{1}^{2} n_{2}^{2} r_{1} r_{2}-504 n_{1}^{4} n_{2}^{2} r_{1} r_{2}+432 n_{1}^{6} n_{2}^{2} r_{1} r_{2}+$ $108 n_{2}^{4} r_{1} r_{2}-504 n_{1}^{2} n_{2}^{4} r_{1} r_{2}+864 n_{1}^{4} n_{2}^{4} r_{1} r_{2}+432 n_{1}^{2} n_{2}^{6} r_{1} r_{2}-144 n_{1}^{2} n_{2}^{2} r_{1}^{2} r_{2}+360 n_{1}^{4} n_{2}^{2} r_{1}^{2} r_{2}-$ $216 n_{1}^{6} n_{2}^{2} r_{1}^{2} r_{2}+360 n_{1}^{2} n_{2}^{4} r_{1}^{2} r_{2}-432 n_{1}^{4} n_{2}^{4} r_{1}^{2} r_{2}-216 n_{1}^{2} n_{2}^{6} r_{1}^{2} r_{2}+72 \dot{\gamma}^{2} \theta_{0}^{2}-36 \dot{\gamma}^{2} n_{1}^{2} \theta_{0}^{2}+18 \dot{\gamma}^{2} n_{1}^{4} \theta_{0}^{2}-$ $36 \dot{\gamma}^{2} n_{2}^{2} \theta_{0}^{2}+36 \dot{\gamma}^{2} n_{1}^{2} n_{2}^{2} \theta_{0}^{2}+18 \dot{\gamma}^{2} n_{2}^{4} \theta_{0}^{2}+72 \dot{\gamma}^{2} n_{1}^{2} r_{1} \theta_{0}^{2}-72 \dot{\gamma}^{2} n_{1}^{4} r_{1} \theta_{0}^{2}+72 \dot{\gamma}^{2} n_{2}^{2} r_{1} \theta_{0}^{2}-$ $144 \dot{\gamma}^{2} n_{1}^{2} n_{2}^{2} r_{1} \theta_{0}^{2}-72 \dot{\gamma}^{2} n_{2}^{4} r_{1} \theta_{0}^{2}-36 \dot{\gamma}^{2} n_{1}^{2} r_{2} \theta_{0}^{2}+54 \dot{\gamma}^{2} n_{1}^{4} r_{2} \theta_{0}^{2}-36 \dot{\gamma}^{2} n_{2}^{2} r_{2} \theta_{0}^{2}+108 \dot{\gamma}^{2} n_{1}^{2} n_{2}^{2} r_{2} \theta_{0}^{2}+$ $\left.\left.\left.54 \dot{\gamma}^{2} n_{2}^{4} r_{2} \theta_{0}^{2}\right)\right)\right) /\left(\left(-2 n_{1} n_{2}+n_{1}^{3} n_{2}+2 n_{1} n_{2} r_{1}-4 n_{1}^{3} n_{2} r_{1}+3 n_{1}^{3} n_{2} r_{2}+\dot{\gamma} \theta_{0}\right)\right.$, 
$N_{1} \rightarrow-\frac{-\dot{\gamma} \eta_{0}-\dot{\gamma} \hat{a} \eta_{0}+3 \dot{\gamma} n_{1}^{2} n_{2}^{2} \eta_{0}+4 \dot{\gamma} \hat{l} n_{1}^{2} n_{2}^{2} \eta_{0}-2 \dot{\gamma} \hat{\beta} n_{1}^{2} n_{2}^{2} \eta_{0}}{-2 n_{1} n_{2}+n_{1}^{3} n_{2}+2 n_{1} n_{2} r_{1}-4 n_{1}^{3} n_{2} r_{1}+3 n_{1}^{3} n_{2} r_{2}+\dot{\gamma} \theta_{0}}+$

$\left(6 \dot{\gamma} \eta_{0}+6 \dot{\gamma} \hat{a} \eta_{0}-9 \dot{\gamma} n_{1}^{2} \eta_{0}-9 \dot{\gamma} \hat{a l} n_{1}^{2} \eta_{0}+3 \dot{\gamma} n_{1}^{4} \eta_{0}+3 \dot{\gamma} \hat{a} n_{1}^{4} \eta_{0}-12 \dot{\gamma} n_{1}^{2} n_{2}^{2} \eta_{0}-12 \dot{\gamma} a \hat{l} n_{1}^{2} n_{2}^{2} \eta_{0}+\right.$ $8 \dot{\gamma} \hat{\beta} n_{1}^{2} n_{2}^{2} \eta_{0}+6 \dot{\gamma} n_{1}^{4} n_{2}^{2} \eta_{0}+6 \dot{\gamma} \hat{a} n_{1}^{4} n_{2}^{2} \eta_{0}-4 \dot{\gamma} \hat{\beta} n_{1}^{4} n_{2}^{2} \eta_{0}+12 \dot{\gamma} n_{1}^{2} r_{1} \eta_{0}+12 \dot{\gamma} a \hat{l} n_{1}^{2} r_{1} \eta_{0}-12 \dot{\gamma} n_{1}^{4} r_{1} \eta_{0}-$ $12 \dot{\gamma} \hat{a l} n_{1}^{4} r_{1} \eta_{0}-6 \dot{\gamma} n_{1}^{2} n_{2}^{2} r_{1} \eta_{0}-12 \dot{\gamma} \hat{a l} n_{1}^{2} n_{2}^{2} r_{1} \eta_{0}+4 \dot{\gamma} \hat{\beta} n_{1}^{2} n_{2}^{2} r_{1} \eta_{0}-6 \dot{\gamma} n_{1}^{4} n_{2}^{2} r_{1} \eta_{0}+4 \dot{\gamma} \hat{\beta} n_{1}^{4} n_{2}^{2} r_{1} \eta_{0}-$ $3 \dot{\gamma} n_{1}^{2} r_{2} \eta_{0}-3 \dot{\gamma} \hat{a} n_{1}^{2} r_{2} \eta_{0}+9 \dot{\gamma} n_{1}^{4} r_{2} \eta_{0}+9 \dot{\gamma}$ al $n_{1}^{4} r_{2} \eta_{0}-6 \dot{\gamma}$ al $n_{1}^{4} n_{2}^{2} r_{2} \eta_{0}-3 \dot{\gamma}^{2} n_{1} n_{2} \eta_{0} \theta_{0}-$ $\left.6 \dot{\gamma}^{2} \hat{a l} n_{1} n_{2} \eta_{0} \theta_{0}+2 \dot{\gamma}^{2} \hat{\beta} n_{1} n_{2} \eta_{0} \theta_{0}+9 \dot{\gamma}^{2} n_{1}^{3} n_{2} \eta_{0} \theta_{0}+12 \dot{\gamma}^{2} a \hat{l} n_{1}^{3} n_{2} \eta_{0} \theta_{0}-6 \dot{\gamma}^{2} \hat{\beta} n_{1}^{3} n_{2} \eta_{0} \theta_{0}\right) /$ $\left(12 n_{1} n_{2}-18 n_{1}^{3} n_{2}+6 n_{1}^{5} n_{2}-12 n_{1} n_{2}^{3}+6 n_{1}^{3} n_{2}^{3}-12 n_{1} n_{2} n_{1}+42 n_{1}^{3} n_{2} r_{1}-30 n_{1}^{5} n_{2} r_{1}+30 n_{1} n_{2}^{3} n_{1}-\right.$ $30 n_{1}^{3} n_{2}^{3} r_{1}-24 n_{1}^{3} n_{2} r_{1}^{2}+24 n_{1}^{5} n_{2} r_{1}^{2}+24 n_{1}^{3} n_{2}^{3} r_{1}^{2}-6 n_{1}^{3} n_{2} r_{2}+18 n_{1}^{5} n_{2} r_{2}-12 n_{1} n_{2}^{3} r_{2}+18 n_{1}^{3} n_{2}^{3} r_{2}+$ $6 n_{1}^{3} n_{2} r_{1} r_{2}-18 n_{1}^{5} n_{2} r_{1} r_{2}-6 n_{1} n_{2}^{3} r_{1} r_{2}-18 n_{1}^{3} n_{2}^{3} r_{1} r_{2}+6 \dot{\gamma} \theta_{0}-9 \dot{\gamma} n_{1}^{2} \theta_{0}+3 \dot{\gamma} n_{1}^{4} \theta_{0}+3 \dot{\gamma} n_{2}^{2} \theta_{0}+$ $\left.3 \dot{\gamma} n_{1}^{2} n_{2}^{2} \theta_{0}+12 \dot{\gamma} n_{1}^{2} r_{1} \theta_{0}-12 \dot{\gamma} n_{1}^{4} r_{1} \theta_{0}-12 \dot{\gamma} n_{1}^{2} n_{2}^{2} r_{1} \theta_{0}-3 \dot{\gamma} n_{1}^{2} r_{2} \theta_{0}+9 \dot{\gamma} n_{1}^{4} r_{2} \theta_{0}-3 \dot{\gamma} n_{2}^{2} r_{2} \theta_{0}+9 \dot{\gamma} n_{1}^{2} n_{2}^{2} r_{2} \theta_{0}\right)+$ $\left(\left(2 n_{1}^{2} n_{2}^{2}+2 r_{1}-8 n_{1}^{2} n_{2}^{2} r_{1}+6 n_{1}^{2} n_{2}^{2} r_{2}\right)\right.$

$\left(-36 \dot{\gamma} \eta_{0}-36 \dot{\gamma} \hat{a} \eta_{0}+54 \dot{\gamma} n_{1}^{2} \eta_{0}+54 \dot{\gamma}\right.$ âl $n_{1}^{2} \eta_{0}-18 \dot{\gamma} n_{1}^{4} \eta_{0}-18 \dot{\gamma}$ all $n_{1}^{4} \eta_{0}+54 \dot{\gamma} n_{2}^{2} \eta_{0}+54 \dot{\gamma} \hat{a l} n_{2}^{2} \eta_{0}-$ $36 \dot{\gamma} n_{1}^{2} n_{2}^{2} \eta_{0}-72 \dot{\gamma} a 1 n_{1}^{2} n_{2}^{2} \eta_{0}-24 \dot{\gamma} \hat{\beta} n_{1}^{2} n_{2}^{2} \eta_{0}+72 \dot{\gamma} n_{1}^{4} n_{2}^{2} \eta_{0}+144 \dot{\gamma}$ al $n_{1}^{4} n_{2}^{2} \eta_{0}-24 \dot{\gamma} \hat{\beta} n_{1}^{4} n_{2}^{2} \eta_{0}-$ $72 \dot{\gamma} n_{1}^{6} n_{2}^{2} \eta_{0}-108 \dot{\gamma} a 1 n_{1}^{6} n_{2}^{2} \eta_{0}+48 \dot{\gamma} \hat{\beta} n_{1}^{6} n_{2}^{2} \eta_{0}-18 \dot{\gamma} n_{2}^{4} \eta_{0}-18 \dot{\gamma} \hat{a} n_{2}^{4} \eta_{0}+72 \dot{\gamma} n_{1}^{2} n_{2}^{4} \eta_{0}+$ $144 \dot{\gamma} \hat{a} n_{1}^{2} n_{2}^{4} \eta_{0}-24 \dot{\gamma} \hat{\beta} n_{1}^{2} n_{2}^{4} \eta_{0}-144 \dot{\gamma} n_{1}^{4} n_{2}^{4} \eta_{0}-216 \dot{\gamma}$ al $n_{1}^{4} n_{2}^{4} \eta_{0}+96 \dot{\gamma} \hat{\beta} n_{1}^{4} n_{2}^{4} \eta_{0}-72 \dot{\gamma} n_{1}^{2} n_{2}^{6} \eta_{0}-$ $108 \dot{\gamma} \hat{a l} n_{1}^{2} n_{2}^{6} \eta_{0}+48 \dot{\gamma} \hat{\beta} n_{1}^{2} n_{2}^{6} \eta_{0}-72 \dot{\gamma} n_{1}^{2} r_{1} \eta_{0}-72 \dot{\gamma} \hat{a} n_{1}^{2} r_{1} \eta_{0}+72 \dot{\gamma} n_{1}^{4} r_{1} \eta_{0}+72 \dot{\gamma} \hat{a l} n_{1}^{4} r_{1} \eta_{0}-$ $72 \dot{\gamma} n_{2}^{2} r_{1} \eta_{0}-72 \dot{\gamma} \hat{a l} n_{2}^{2} r_{1} \eta_{0}+288 \dot{\gamma} n_{1}^{2} n_{2}^{2} r_{1} \eta_{0}+360 \dot{\gamma} \hat{a l} n_{1}^{2} n_{2}^{2} r_{1} \eta_{0}-48 \dot{\gamma} \hat{\beta} n_{1}^{2} n_{2}^{2} r_{1} \eta_{0}-$ $288 \dot{\gamma} n_{1}^{4} n_{2}^{2} r_{1} \eta_{0}-468 \dot{\gamma}$ âl $n_{1}^{4} n_{2}^{2} r_{1} \eta_{0}+72 \dot{\gamma} \hat{\beta} n_{1}^{4} n_{2}^{2} r_{1} \eta_{0}+144 \dot{\gamma} n_{1}^{6} n_{2}^{2} r_{1} \eta_{0}+252 \dot{\gamma}$ al $n_{1}^{6} n_{2}^{2} r_{1} \eta_{0}-$ $96 \dot{\gamma} \hat{\beta} n_{1}^{6} n_{2}^{2} r_{1} \eta_{0}+72 \dot{\gamma} n_{2}^{4} r_{1} \eta_{0}+72 \dot{\gamma} a \hat{l} n_{2}^{4} r_{1} \eta_{0}-288 \dot{\gamma} n_{1}^{2} n_{2}^{4} r_{1} \eta_{0}-468 \dot{\gamma} a 1 n_{1}^{2} n_{2}^{4} r_{1} \eta_{0}+$ $72 \dot{\gamma} \hat{\beta} n_{1}^{2} n_{2}^{4} r_{1} \eta_{0}+288 \dot{\gamma} n_{1}^{4} n_{2}^{4} r_{1} \eta_{0}+504 \dot{\gamma} \hat{a} n_{1}^{4} n_{2}^{4} r_{1} \eta_{0}-192 \dot{\gamma} \hat{\beta} n_{1}^{4} n_{2}^{4} r_{1} \eta_{0}+144 \dot{\gamma} n_{1}^{2} n_{2}^{6} r_{1} \eta_{0}+$ $252 \dot{\gamma} \hat{a} 1 n_{1}^{2} n_{2}^{6} r_{1} \eta_{0}-96 \dot{\gamma} \hat{\beta} n_{1}^{2} n_{2}^{6} r_{1} \eta_{0}-144 \dot{\gamma} n_{1}^{2} n_{2}^{2} r_{1}^{2} \eta_{0}-144 \dot{\gamma}$ al $n_{1}^{2} n_{2}^{2} r_{1}^{2} \eta_{0}+216 \dot{\gamma} n_{1}^{4} n_{2}^{2} r_{1}^{2} \eta_{0}+$ $288 \dot{\gamma} a 1 n_{1}^{4} n_{2}^{2} r_{1}^{2} \eta_{0}-48 \dot{\gamma} \hat{\beta} n_{1}^{4} n_{2}^{2} r_{1}^{2} \eta_{0}-72 \dot{\gamma} n_{1}^{6} n_{2}^{2} r_{1}^{2} \eta_{0}-144 \dot{\gamma}$ al $n_{1}^{6} n_{2}^{2} r_{1}^{2} \eta_{0}+48 \dot{\gamma} \hat{\beta} n_{1}^{6} n_{2}^{2} r_{1}^{2} \eta_{0}+$ $216 \dot{\gamma} n_{1}^{2} n_{2}^{4} r_{1}^{2} \eta_{0}+288 \dot{\gamma}$ al $n_{1}^{2} n_{2}^{4} r_{1}^{2} \eta_{0}-48 \dot{\gamma} \hat{\beta} n_{1}^{2} n_{2}^{4} r_{1}^{2} \eta_{0}-144 \dot{\gamma} n_{1}^{4} n_{2}^{4} r_{1}^{2} \eta_{0}-288 \dot{\gamma}$ al $n_{1}^{4} n_{2}^{4} r_{1}^{2} \eta_{0}+$ $96 \dot{\gamma} \hat{\beta} n_{1}^{4} n_{2}^{4} r_{1}^{2} \eta_{0}-72 \dot{\gamma} n_{1}^{2} n_{2}^{6} r_{1}^{2} \eta_{0}-144 \dot{\gamma} \hat{a l} n_{1}^{2} n_{2}^{6} r_{1}^{2} \eta_{0}+48 \dot{\gamma} \hat{\beta} n_{1}^{2} n_{2}^{6} r_{1}^{2} \eta_{0}+18 \dot{\gamma} n_{1}^{2} r_{2} \eta_{0}+$ $18 \dot{\gamma} \hat{a} n_{1}^{2} r_{2} \eta_{0}-54 \dot{\gamma} n_{1}^{4} r_{2} \eta_{0}-54 \dot{\gamma}$ all $n_{1}^{4} r_{2} \eta_{0}+18 \dot{\gamma} n_{2}^{2} r_{2} \eta_{0}+18 \dot{\gamma}$ âl $n_{2}^{2} r_{2} \eta_{0}-72 \dot{\gamma} n_{1}^{2} n_{2}^{2} r_{2} \eta_{0}-$ $72 \dot{\gamma}$ al $n_{1}^{2} n_{2}^{2} r_{2} \eta_{0}+108 \dot{\gamma} n_{1}^{4} n_{2}^{2} r_{2} \eta_{0}+144 \dot{\gamma}$ al $n_{1}^{4} n_{2}^{2} r_{2} \eta_{0}-36 \dot{\gamma}$ al $n_{1}^{6} n_{2}^{2} r_{2} \eta_{0}-54 \dot{\gamma} n_{2}^{4} r_{2} \eta_{0}-$ $54 \dot{\gamma}$ âl $n_{2}^{4} r_{2} \eta_{0}+108 \dot{\gamma} n_{1}^{2} n_{2}^{4} r_{2} \eta_{0}+144 \dot{\gamma}$ âl $n_{1}^{2} n_{2}^{4} r_{2} \eta_{0}-72 \dot{\gamma}$ al $n_{1}^{4} n_{2}^{4} r_{2} \eta_{0}-36 \dot{\gamma}$ al $n_{1}^{2} n_{2}^{6} r_{2} \eta_{0}+$ $72 \dot{\gamma} n_{1}^{2} n_{2}^{2} r_{1} r_{2} \eta_{0}+72 \dot{\gamma}$ all $n_{1}^{2} n_{2}^{2} r_{1} r_{2} \eta_{0}-108 \dot{\gamma} n_{1}^{4} n_{2}^{2} r_{1} r_{2} \eta_{0}-108 \dot{\gamma}$ all $n_{1}^{4} n_{2}^{2} r_{1} r_{2} \eta_{0}+$ $36 \dot{\gamma}$ al $n_{1}^{6} n_{2}^{2} r_{1} r_{2} \eta_{0}-108 \dot{\gamma} n_{1}^{2} n_{2}^{4} r_{1} r_{2} \eta_{0}-108 \dot{\gamma}$ al $n_{1}^{2} n_{2}^{4} r_{1} r_{2} \eta_{0}+72 \dot{\gamma}$ all $n_{1}^{4} n_{2}^{4} r_{1} r_{2} \eta_{0}+$ $36 \dot{\gamma} \hat{a l} n_{1}^{2} n_{2}^{6} r_{1} r_{2} \eta_{0}-18 \dot{\gamma}^{2} n_{1}^{3} n_{2} \eta_{0} \theta_{0}+12 \dot{\gamma}^{2} \hat{\beta} n_{1}^{3} n_{2} \eta_{0} \theta_{0}-36 \dot{\gamma}^{2} n_{1}^{5} n_{2} \eta_{0} \theta_{0}-54 \dot{\gamma}^{2} \hat{a l} n_{1}^{5} n_{2} \eta_{0} \theta_{0}+$ $24 \dot{\gamma}^{2} \hat{\beta} n_{1}^{5} n_{2} \eta_{0} \theta_{0}+18 \dot{\gamma}^{2} n_{1} n_{2}^{3} \eta_{0} \theta_{0}-12 \dot{\gamma}^{2} \hat{\beta} n_{1} n_{2}^{3} \eta_{0} \theta_{0}+36 \dot{\gamma}^{2} n_{1} n_{2}^{5} \eta_{0} \theta_{0}+54 \dot{\gamma}^{2}$ al $n_{1} n_{2}^{5} \eta_{0} \theta_{0}-$ $24 \dot{\gamma}^{2} \hat{\beta} n_{1} n_{2}^{5} \eta_{0} \theta_{0}-36 \dot{\gamma}^{2} n_{1}^{3} n_{2} r_{1} \eta_{0} \theta_{0}-72 \dot{\gamma}^{2} \hat{a l} n_{1}^{3} n_{2} r_{1} \eta_{0} \theta_{0}+24 \dot{\gamma}^{2} \hat{\beta} n_{1}^{3} n_{2} r_{1} \eta_{0} \theta_{0}+36 \dot{\gamma}^{2} n_{1}^{5} n_{2} r_{1} \eta_{0} \theta_{0}+$ $72 \dot{\gamma}^{2} \hat{a} n_{1}^{5} n_{2} r_{1} \eta_{0} \theta_{0}-24 \dot{\gamma}^{2} \hat{\beta} n_{1}^{5} n_{2} r_{1} \eta_{0} \theta_{0}+36 \dot{\gamma}^{2} n_{1} n_{2}^{3} r_{1} \eta_{0} \theta_{0}+72 \dot{\gamma}^{2} \hat{a} n_{1} n_{2}^{3} r_{1} \eta_{0} \theta_{0}-$ $24 \dot{\gamma}^{2} \hat{\beta} n_{1} n_{2}^{3} r_{1} \eta_{0} \theta_{0}-36 \dot{\gamma}^{2} n_{1} n_{2}^{5} r_{1} \eta_{0} \theta_{0}-72 \dot{\gamma}^{2} \hat{a} n_{1} n_{2}^{5} r_{1} \eta_{0} \theta_{0}+24 \dot{\gamma}^{2} \hat{\beta} n_{1} n_{2}^{5} r_{1} \eta_{0} \theta_{0}-$ $18 \dot{\gamma}^{2} a \hat{1} n_{1}^{5} n_{2} r_{2} \eta_{0} \theta_{0}+18 \dot{\gamma}^{2}$ âl $\left.\left.n_{1} n_{2}^{5} r_{2} \eta_{0} \theta_{0}\right)\right) /$

$\left(\left(-2 n_{1} n_{2}+n_{1}^{3} n_{2}+2 n_{1} n_{2} n_{1}-4 n_{1}^{3} n_{2} n_{1}+3 n_{1}^{3} n_{2} r_{2}+\dot{\gamma} \theta_{0}\right)\right.$

$\left(-72 n_{1}^{2} n_{2}^{2}+144 n_{1}^{4} n_{2}^{2}-72 n_{1}^{6} n_{2}^{2}+144 n_{1}^{2} n_{2}^{4}-144 n_{1}^{4} n_{2}^{4}-72 n_{1}^{2} n_{2}^{6}+72 n_{1}-108 n_{1}^{2} r_{1}+36 n_{1}^{4} r_{1}-108 n_{2}^{2} r_{1}+\right.$ $288 n_{1}^{2} n_{2}^{2} r_{1}-648 n_{1}^{4} n_{2}^{2} r_{1}+432 n_{1}^{6} n_{2}^{2} r_{1}+36 n_{2}^{4} r_{1}-648 n_{1}^{2} n_{2}^{4} r_{1}+864 n_{1}^{4} n_{2}^{4} r_{1}+432 n_{1}^{2} n_{2}^{6} r_{1}+144 n_{1}^{2} r_{1}^{2}-$ $144 n_{1}^{4} r_{1}^{2}+144 n_{2}^{2} r_{1}^{2}-720 n_{1}^{2} n_{2}^{2} r_{1}^{2}+1080 n_{1}^{4} n_{2}^{2} r_{1}^{2}-648 n_{1}^{6} n_{2}^{2} r_{1}^{2}-144 n_{2}^{4} r_{1}^{2}+1080 n_{1}^{2} n_{2}^{4} r_{1}^{2}-1296 n_{1}^{4} n_{2}^{4} r_{1}^{2}-$ $648 n_{1}^{2} n_{2}^{6} r_{1}^{2}+288 n_{1}^{2} n_{2}^{2} r_{1}^{3}-576 n_{1}^{4} n_{2}^{2} r_{1}^{3}+288 n_{1}^{6} n_{2}^{2} r_{1}^{3}-576 n_{1}^{2} n_{2}^{4} r_{1}^{3}+576 n_{1}^{4} n_{2}^{4} r_{1}^{3}+288 n_{1}^{2} n_{2}^{6} r_{1}^{3}+$ $72 n_{1}^{2} n_{2}^{2} r_{2}+144 n_{1}^{4} n_{2}^{2} r_{2}-216 n_{1}^{6} n_{2}^{2} r_{2}+144 n_{1}^{2} n_{2}^{4} r_{2}-432 n_{1}^{4} n_{2}^{4} r_{2}-216 n_{1}^{2} n_{2}^{6} r_{2}-36 n_{1}^{2} r_{1} r_{2}+$ $108 n_{1}^{4} r_{1} r_{2}-36 n_{2}^{2} r_{1} r_{2}+288 n_{1}^{2} n_{2}^{2} r_{1} r_{2}-504 n_{1}^{4} n_{2}^{2} r_{1} r_{2}+432 n_{1}^{6} n_{2}^{2} r_{1} r_{2}+108 n_{2}^{4} r_{1} r_{2}-504 n_{1}^{2} n_{2}^{4} r_{1} r_{2}+$ $864 n_{1}^{4} n_{2}^{4} r_{1} r_{2}+432 n_{1}^{2} n_{2}^{6} r_{1} r_{2}-144 n_{1}^{2} n_{2}^{2} r_{1}^{2} r_{2}+360 n_{1}^{4} n_{2}^{2} r_{1}^{2} r_{2}-216 n_{1}^{6} n_{2}^{2} r_{1}^{2} r_{2}+360 n_{1}^{2} n_{2}^{4} r_{1}^{2} r_{2}-$ $432 n_{1}^{4} n_{2}^{4} r_{1}^{2} r_{2}-216 n_{1}^{2} n_{2}^{6} r_{1}^{2} r_{2}+72 \dot{\gamma}^{2} \theta_{0}^{2}-36 \dot{\gamma}^{2} n_{1}^{2} \theta_{0}^{2}+18 \dot{\gamma}^{2} n_{1}^{4} \theta_{0}^{2}-36 \dot{\gamma}^{2} n_{2}^{2} \theta_{0}^{2}+36 \dot{\gamma}^{2} n_{1}^{2} n_{2}^{2} \theta_{0}^{2}+$ $18 \dot{\gamma}^{2} n_{2}^{4} \theta_{0}^{2}+72 \dot{\gamma}^{2} n_{1}^{2} r_{1} \theta_{0}^{2}-72 \dot{\gamma}^{2} n_{1}^{4} r_{1} \theta_{0}^{2}+72 \dot{\gamma}^{2} n_{2}^{2} r_{1} \theta_{0}^{2}-144 \dot{\gamma}^{2} n_{1}^{2} n_{2}^{2} r_{1} \theta_{0}^{2}-72 \dot{\gamma}^{2} n_{2}^{4} r_{1} \theta_{0}^{2}-$ $\left.\left.36 \dot{\gamma}^{2} n_{1}^{2} r_{2} \theta_{0}^{2}+54 \dot{\gamma}^{2} n_{1}^{4} r_{2} \theta_{0}^{2}-36 \dot{\gamma}^{2} n_{2}^{2} r_{2} \theta_{0}^{2}+108 \dot{\gamma}^{2} n_{1}^{2} n_{2}^{2} r_{2} \theta_{0}^{2}+54 \dot{\gamma}^{2} n_{2}^{4} r_{2} \theta_{0}^{2}\right)\right)-$ 
$\left(\left(-36 \dot{\gamma} \eta_{0}-36 \dot{\gamma}\right.\right.$ all $\eta_{0}+54 \dot{\gamma} n_{1}^{2} \eta_{0}+54 \dot{\gamma}$ âl $n_{1}^{2} \eta_{0}-18 \dot{\gamma} n_{1}^{4} \eta_{0}-18 \dot{\gamma}$ âl $n_{1}^{4} \eta_{0}+54 \dot{\gamma} n_{2}^{2} \eta_{0}+54 \dot{\gamma}$ all $n_{2}^{2} \eta_{0}-$ $36 \dot{\gamma} n_{1}^{2} n_{2}^{2} \eta_{0}-72 \dot{\gamma} a 1 n_{1}^{2} n_{2}^{2} \eta_{0}-24 \dot{\gamma} \hat{\beta} n_{1}^{2} n_{2}^{2} \eta_{0}+72 \dot{\gamma} n_{1}^{4} n_{2}^{2} \eta_{0}+144 \dot{\gamma} a \hat{l} n_{1}^{4} n_{2}^{2} \eta_{0}-24 \dot{\gamma} \hat{\beta} n_{1}^{4} n_{2}^{2} \eta_{0}-$ $72 \dot{\gamma} n_{1}^{6} n_{2}^{2} \eta_{0}-108 \dot{\gamma} \hat{a l} n_{1}^{6} n_{2}^{2} \eta_{0}+48 \dot{\gamma} \hat{\beta} n_{1}^{6} n_{2}^{2} \eta_{0}-18 \dot{\gamma} n_{2}^{4} \eta_{0}-18 \dot{\gamma} \hat{a} 1 n_{2}^{4} \eta_{0}+72 \dot{\gamma} n_{1}^{2} n_{2}^{4} \eta_{0}+144 \dot{\gamma} a \hat{l} n_{1}^{2} n_{2}^{4} \eta_{0}-$ $24 \dot{\gamma} \hat{\beta} n_{1}^{2} n_{2}^{4} \eta_{0}-144 \dot{\gamma} n_{1}^{4} n_{2}^{4} \eta_{0}-216 \dot{\gamma} \hat{a} 1 n_{1}^{4} n_{2}^{4} \eta_{0}+96 \dot{\gamma} \hat{\beta} n_{1}^{4} n_{2}^{4} \eta_{0}-72 \dot{\gamma} n_{1}^{2} n_{2}^{6} \eta_{0}-108 \dot{\gamma} \hat{a} n_{1}^{2} n_{2}^{6} \eta_{0}+$ $48 \dot{\gamma} \hat{\beta} n_{1}^{2} n_{2}^{6} \eta_{0}-72 \dot{\gamma} n_{1}^{2} r_{1} \eta_{0}-72 \dot{\gamma}$ all $n_{1}^{2} r_{1} \eta_{0}+72 \dot{\gamma} n_{1}^{4} r_{1} \eta_{0}+72 \dot{\gamma}$ al $n_{1}^{4} r_{1} \eta_{0}-72 \dot{\gamma} n_{2}^{2} r_{1} \eta_{0}-$ $72 \dot{\gamma} a \hat{l} n_{2}^{2} r_{1} \eta_{0}+288 \dot{\gamma} n_{1}^{2} n_{2}^{2} r_{1} \eta_{0}+360 \dot{\gamma}$ al $n_{1}^{2} n_{2}^{2} r_{1} \eta_{0}-48 \dot{\gamma} \hat{\beta} n_{1}^{2} n_{2}^{2} r_{1} \eta_{0}-288 \dot{\gamma} n_{1}^{4} n_{2}^{2} r_{1} \eta_{0}-$ $468 \dot{\gamma} a \hat{l} n_{1}^{4} n_{2}^{2} r_{1} \eta_{0}+72 \dot{\gamma} \hat{\beta} n_{1}^{4} n_{2}^{2} r_{1} \eta_{0}+144 \dot{\gamma} n_{1}^{6} n_{2}^{2} r_{1} \eta_{0}+252 \dot{\gamma}$ all $n_{1}^{6} n_{2}^{2} r_{1} \eta_{0}-96 \dot{\gamma} \hat{\beta} n_{1}^{6} n_{2}^{2} r_{1} \eta_{0}+$ $72 \dot{\gamma} n_{2}^{4} r_{1} \eta_{0}+72 \dot{\gamma} a \hat{l} n_{2}^{4} r_{1} \eta_{0}-288 \dot{\gamma} n_{1}^{2} n_{2}^{4} r_{1} \eta_{0}-468 \dot{\gamma} a \hat{l} n_{1}^{2} n_{2}^{4} r_{1} \eta_{0}+72 \dot{\gamma} \hat{\beta} n_{1}^{2} n_{2}^{4} r_{1} \eta_{0}+288 \dot{\gamma} n_{1}^{4} n_{2}^{4} r_{1} \eta_{0}+$ $504 \dot{\gamma} a \hat{l} n_{1}^{4} n_{2}^{4} r_{1} \eta_{0}-192 \dot{\gamma} \hat{\beta} n_{1}^{4} n_{2}^{4} r_{1} \eta_{0}+144 \dot{\gamma} n_{1}^{2} n_{2}^{6} r_{1} \eta_{0}+252 \dot{\gamma}$ al $n_{1}^{2} n_{2}^{6} r_{1} \eta_{0}-96 \dot{\gamma} \hat{\beta} n_{1}^{2} n_{2}^{6} n_{1} \eta_{0}-$ $144 \dot{\gamma} n_{1}^{2} n_{2}^{2} r_{1}^{2} \eta_{0}-144 \dot{\gamma} \hat{a l} n_{1}^{2} n_{2}^{2} r_{1}^{2} \eta_{0}+216 \dot{\gamma} n_{1}^{4} n_{2}^{2} r_{1}^{2} \eta_{0}+288 \dot{\gamma}$ all $n_{1}^{4} n_{2}^{2} r_{1}^{2} \eta_{0}-48 \dot{\gamma} \hat{\beta} n_{1}^{4} n_{2}^{2} r_{1}^{2} \eta_{0}-$ $72 \dot{\gamma} n_{1}^{6} n_{2}^{2} r_{1}^{2} \eta_{0}-144 \dot{\gamma} a \hat{l} n_{1}^{6} n_{2}^{2} r_{1}^{2} \eta_{0}+48 \dot{\gamma} \hat{\beta} n_{1}^{6} n_{2}^{2} r_{1}^{2} \eta_{0}+216 \dot{\gamma} n_{1}^{2} n_{2}^{4} r_{1}^{2} \eta_{0}+288 \dot{\gamma}$ al $n_{1}^{2} n_{2}^{4} r_{1}^{2} \eta_{0}-$ $48 \dot{\gamma} \hat{\beta} n_{1}^{2} n_{2}^{4} r_{1}^{2} \eta_{0}-144 \dot{\gamma} n_{1}^{4} n_{2}^{4} r_{1}^{2} \eta_{0}-288 \dot{\gamma} \hat{a} n_{1}^{4} n_{2}^{4} r_{1}^{2} \eta_{0}+96 \dot{\gamma} \hat{\beta} n_{1}^{4} n_{2}^{4} r_{1}^{2} \eta_{0}-72 \dot{\gamma} n_{1}^{2} n_{2}^{6} r_{1}^{2} \eta_{0}-$ $144 \dot{\gamma} \hat{a l} n_{1}^{2} n_{2}^{6} r_{1}^{2} \eta_{0}+48 \dot{\gamma} \hat{\beta} n_{1}^{2} n_{2}^{6} r_{1}^{2} \eta_{0}+18 \dot{\gamma} n_{1}^{2} r_{2} \eta_{0}+18 \dot{\gamma} \hat{a} n_{1}^{2} r_{2} \eta_{0}-54 \dot{\gamma} n_{1}^{4} r_{2} \eta_{0}-54 \dot{\gamma} \hat{a} n_{1}^{4} r_{2} \eta_{0}+$ $18 \dot{\gamma} n_{2}^{2} r_{2} \eta_{0}+18 \dot{\gamma} \hat{a l} n_{2}^{2} r_{2} \eta_{0}-72 \dot{\gamma} n_{1}^{2} n_{2}^{2} r_{2} \eta_{0}-72 \dot{\gamma}$ al $n_{1}^{2} n_{2}^{2} r_{2} \eta_{0}+108 \dot{\gamma} n_{1}^{4} n_{2}^{2} r_{2} \eta_{0}+144 \dot{\gamma} \hat{a} 1 n_{1}^{4} n_{2}^{2} r_{2} \eta_{0}-$ $36 \dot{\gamma}$ al $n_{1}^{6} n_{2}^{2} r_{2} \eta_{0}-54 \dot{\gamma} n_{2}^{4} r_{2} \eta_{0}-54 \dot{\gamma}$ al $n_{2}^{4} r_{2} \eta_{0}+108 \dot{\gamma} n_{1}^{2} n_{2}^{4} r_{2} \eta_{0}+144 \dot{\gamma}$ al $n_{1}^{2} n_{2}^{4} r_{2} \eta_{0}-$ $72 \dot{\gamma} \hat{a} n_{1}^{4} n_{2}^{4} r_{2} \eta_{0}-36 \dot{\gamma}$ âl $n_{1}^{2} n_{2}^{6} r_{2} \eta_{0}+72 \dot{\gamma} n_{1}^{2} n_{2}^{2} r_{1} r_{2} \eta_{0}+72 \dot{\gamma} \hat{a l} n_{1}^{2} n_{2}^{2} r_{1} r_{2} \eta_{0}-108 \dot{\gamma} n_{1}^{4} n_{2}^{2} r_{1} r_{2} \eta_{0}-$ $108 \dot{\gamma} \hat{a l} n_{1}^{4} n_{2}^{2} r_{1} r_{2} \eta_{0}+36 \dot{\gamma} \hat{a l l} n_{1}^{6} n_{2}^{2} r_{1} r_{2} \eta_{0}-108 \dot{\gamma} n_{1}^{2} n_{2}^{4} r_{1} r_{2} \eta_{0}-108 \dot{\gamma} \hat{a l} n_{1}^{2} n_{2}^{4} r_{1} r_{2} \eta_{0}+$ $72 \dot{\gamma} \hat{a} n_{1}^{4} n_{2}^{4} n_{1} r_{2} \eta_{0}+36 \dot{\gamma} \hat{a l} n_{1}^{2} n_{2}^{6} n_{1} r_{2} \eta_{0}-18 \dot{\gamma}^{2} n_{1}^{3} n_{2} \eta_{0} \theta_{0}+12 \dot{\gamma}^{2} \hat{\beta} n_{1}^{3} n_{2} \eta_{0} \theta_{0}-36 \dot{\gamma}^{2} n_{1}^{5} n_{2} \eta_{0} \theta_{0}-$ $54 \dot{\gamma}^{2} a \hat{1} n_{1}^{5} n_{2} \eta_{0} \theta_{0}+24 \dot{\gamma}^{2} \hat{\beta} n_{1}^{5} n_{2} \eta_{0} \theta_{0}+18 \dot{\gamma}^{2} n_{1} n_{2}^{3} \eta_{0} \theta_{0}-12 \dot{\gamma}^{2} \hat{\beta} n_{1} n_{2}^{3} \eta_{0} \theta_{0}+36 \dot{\gamma}^{2} n_{1} n_{2}^{5} \eta_{0} \theta_{0}+$ $54 \dot{\gamma}^{2} \hat{a} 1 n_{1} n_{2}^{5} \eta_{0} \theta_{0}-24 \dot{\gamma}^{2} \hat{\beta} n_{1} n_{2}^{5} \eta_{0} \theta_{0}-36 \dot{\gamma}^{2} n_{1}^{3} n_{2} r_{1} \eta_{0} \theta_{0}-72 \dot{\gamma}^{2} \hat{a} 1 n_{1}^{3} n_{2} n_{1} \eta_{0} \theta_{0}+24 \dot{\gamma}^{2} \hat{\beta} n_{1}^{3} n_{2} r_{1} \eta_{0} \theta_{0}+$ $36 \dot{\gamma}^{2} n_{1}^{5} n_{2} r_{1} \eta_{0} \theta_{0}+72 \dot{\gamma}^{2} \hat{a} n_{1}^{5} n_{2} r_{1} \eta_{0} \theta_{0}-24 \dot{\gamma}^{2} \hat{\beta} n_{1}^{5} n_{2} r_{1} \eta_{0} \theta_{0}+36 \dot{\gamma}^{2} n_{1} n_{2}^{3} n_{1} \eta_{0} \theta_{0}+72 \dot{\gamma}^{2} \hat{a} 1 n_{1} n_{2}^{3} n_{1} \eta_{0} \theta_{0}-$ $24 \dot{\gamma}^{2} \hat{\beta} n_{1} n_{2}^{3} r_{1} \eta_{0} \theta_{0}-36 \dot{\gamma}^{2} n_{1} n_{2}^{5} r_{1} \eta_{0} \theta_{0}-72 \dot{\gamma}^{2} \hat{a} n_{1} n_{2}^{5} r_{1} \eta_{0} \theta_{0}+24 \dot{\gamma}^{2} \hat{\beta} n_{1} n_{2}^{5} r_{1} \eta_{0} \theta_{0}-$ $\left.18 \dot{\gamma}^{2} \hat{a} \hat{l} n_{1}^{5} n_{2} r_{2} \eta_{0} \theta_{0}+18 \dot{\gamma}^{2} \hat{a l} n_{1} n_{2}^{5} r_{2} \eta_{0} \theta_{0}\right)$

$\left(-12 r_{1}+18 n_{1}^{2} r_{1}-6 n_{1}^{4} r_{1}+12 n_{1}^{2} n_{2}^{2} r_{1}-24 n_{1}^{2} r_{1}^{2}+24 n_{1}^{4} r_{1}^{2}+24 n_{1}^{2} n_{2}^{2} r_{1}^{2}-24 n_{1}^{2} n_{2}^{2} r_{2}+6 n_{1}^{2} r_{1} r_{2}-\right.$ $\left.\left.18 n_{1}^{4} r_{1} r_{2}-12 n_{1}^{2} n_{2}^{2} r_{1} r_{2}+6 \dot{\gamma} n_{1} n_{2} \theta_{0}-6 \dot{\gamma} n_{1} n_{2} r_{2} \theta_{0}-6 \dot{\gamma}^{2} \theta_{0}^{2}\right)\right) /$

( $\left(12 n_{1} n_{2}-18 n_{1}^{3} n_{2}+6 n_{1}^{5} n_{2}-12 n_{1} n_{2}^{3}+6 n_{1}^{3} n_{2}^{3}-12 n_{1} n_{2} r_{1}+42 n_{1}^{3} n_{2} n_{1}-30 n_{1}^{5} n_{2} n_{1}+30 n_{1} n_{2}^{3} n_{1}-\right.$ $30 n_{1}^{3} n_{2}^{3} r_{1}-24 n_{1}^{3} n_{2} r_{1}^{2}+24 n_{1}^{5} n_{2} r_{1}^{2}+24 n_{1}^{3} n_{2}^{3} r_{1}^{2}-6 n_{1}^{3} n_{2} r_{2}+18 n_{1}^{5} n_{2} r_{2}-12 n_{1} n_{2}^{3} r_{2}+18 n_{1}^{3} n_{2}^{3} r_{2}+$ $6 n_{1}^{3} n_{2} r_{1} r_{2}-18 n_{1}^{5} n_{2} n_{1} r_{2}-6 n_{1} n_{2}^{3} n_{1} r_{2}-18 n_{1}^{3} n_{2}^{3} r_{1} r_{2}+6 \dot{\gamma} \theta_{0}-9 \dot{\gamma} n_{1}^{2} \theta_{0}+3 \dot{\gamma} n_{1}^{4} \theta_{0}+3 \dot{\gamma} n_{2}^{2} \theta_{0}+$ $\left.3 \dot{\gamma} n_{1}^{2} n_{2}^{2} \theta_{0}+12 \dot{\gamma} n_{1}^{2} r_{1} \theta_{0}-12 \dot{\gamma} n_{1}^{4} r_{1} \theta_{0}-12 \dot{\gamma} n_{1}^{2} n_{2}^{2} r_{1} \theta_{0}-3 \dot{\gamma} n_{1}^{2} r_{2} \theta_{0}+9 \dot{\gamma} n_{1}^{4} r_{2} \theta_{0}-3 \dot{\gamma} n_{2}^{2} r_{2} \theta_{0}+9 \dot{\gamma} n_{1}^{2} n_{2}^{2} r_{2} \theta_{0}\right)$ $\left(-72 n_{1}^{2} n_{2}^{2}+144 n_{1}^{4} n_{2}^{2}-72 n_{1}^{6} n_{2}^{2}+144 n_{1}^{2} n_{2}^{4}-144 n_{1}^{4} n_{2}^{4}-72 n_{1}^{2} n_{2}^{6}+72 r_{1}-108 n_{1}^{2} r_{1}+36 n_{1}^{4} r_{1}-108 n_{2}^{2} r_{1}+\right.$ $288 n_{1}^{2} n_{2}^{2} r_{1}-648 n_{1}^{4} n_{2}^{2} r_{1}+432 n_{1}^{6} n_{2}^{2} r_{1}+36 n_{2}^{4} r_{1}-648 n_{1}^{2} n_{2}^{4} r_{1}+864 n_{1}^{4} n_{2}^{4} r_{1}+432 n_{1}^{2} n_{2}^{6} r_{1}+144 n_{1}^{2} r_{1}^{2}-$ $144 n_{1}^{4} r_{1}^{2}+144 n_{2}^{2} r_{1}^{2}-720 n_{1}^{2} n_{2}^{2} r_{1}^{2}+1080 n_{1}^{4} n_{2}^{2} r_{1}^{2}-648 n_{1}^{6} n_{2}^{2} r_{1}^{2}-144 n_{2}^{4} r_{1}^{2}+1080 n_{1}^{2} n_{2}^{4} r_{1}^{2}-1296 n_{1}^{4} n_{2}^{4} r_{1}^{2}-$ $648 n_{1}^{2} n_{2}^{6} r_{1}^{2}+288 n_{1}^{2} n_{2}^{2} r_{1}^{3}-576 n_{1}^{4} n_{2}^{2} r_{1}^{3}+288 n_{1}^{6} n_{2}^{2} r_{1}^{3}-576 n_{1}^{2} n_{2}^{4} r_{1}^{3}+576 n_{1}^{4} n_{2}^{4} r_{1}^{3}+288 n_{1}^{2} n_{2}^{6} r_{1}^{3}+$ $72 n_{1}^{2} n_{2}^{2} r_{2}+144 n_{1}^{4} n_{2}^{2} r_{2}-216 n_{1}^{6} n_{2}^{2} r_{2}+144 n_{1}^{2} n_{2}^{4} r_{2}-432 n_{1}^{4} n_{2}^{4} r_{2}-216 n_{1}^{2} n_{2}^{6} r_{2}-36 n_{1}^{2} r_{1} r_{2}+$ $108 n_{1}^{4} r_{1} r_{2}-36 n_{2}^{2} r_{1} r_{2}+288 n_{1}^{2} n_{2}^{2} r_{1} r_{2}-504 n_{1}^{4} n_{2}^{2} r_{1} r_{2}+432 n_{1}^{6} n_{2}^{2} r_{1} r_{2}+108 n_{2}^{4} r_{1} r_{2}-504 n_{1}^{2} n_{2}^{4} r_{1} r_{2}+$ $864 n_{1}^{4} n_{2}^{4} r_{1} r_{2}+432 n_{1}^{2} n_{2}^{6} r_{1} r_{2}-144 n_{1}^{2} n_{2}^{2} r_{1}^{2} r_{2}+360 n_{1}^{4} n_{2}^{2} r_{1}^{2} r_{2}-216 n_{1}^{6} n_{2}^{2} r_{1}^{2} r_{2}+360 n_{1}^{2} n_{2}^{4} r_{1}^{2} r_{2}-$ $432 n_{1}^{4} n_{2}^{4} r_{1}^{2} r_{2}-216 n_{1}^{2} n_{2}^{6} r_{1}^{2} r_{2}+72 \dot{\gamma}^{2} \theta_{0}^{2}-36 \dot{\gamma}^{2} n_{1}^{2} \theta_{0}^{2}+18 \dot{\gamma}^{2} n_{1}^{4} \theta_{0}^{2}-36 \dot{\gamma}^{2} n_{2}^{2} \theta_{0}^{2}+36 \dot{\gamma}^{2} n_{1}^{2} n_{2}^{2} \theta_{0}^{2}+$ $18 \dot{\gamma}^{2} n_{2}^{4} \theta_{0}^{2}+72 \dot{\gamma}^{2} n_{1}^{2} r_{1} \theta_{0}^{2}-72 \dot{\gamma}^{2} n_{1}^{4} r_{1} \theta_{0}^{2}+72 \dot{\gamma}^{2} n_{2}^{2} r_{1} \theta_{0}^{2}-144 \dot{\gamma}^{2} n_{1}^{2} n_{2}^{2} r_{1} \theta_{0}^{2}-72 \dot{\gamma}^{2} n_{2}^{4} r_{1} \theta_{0}^{2}-$ $\left.\left.36 \dot{\gamma}^{2} n_{1}^{2} r_{2} \theta_{0}^{2}+54 \dot{\gamma}^{2} n_{1}^{4} r_{2} \theta_{0}^{2}-36 \dot{\gamma}^{2} n_{2}^{2} r_{2} \theta_{0}^{2}+108 \dot{\gamma}^{2} n_{1}^{2} n_{2}^{2} r_{2} \theta_{0}^{2}+54 \dot{\gamma}^{2} n_{2}^{4} r_{2} \theta_{0}^{2}\right)\right)+$

$\left(\left(-2 n_{1} n_{2}+n_{1} n_{2}^{3}+2 n_{1} n_{2} r_{1}-4 n_{1} n_{2}^{3} r_{1}+3 n_{1} n_{2}^{3} r_{2}-\dot{\gamma} \theta_{0}\right)\right.$

$\left(\left(6 \dot{\gamma} \eta_{0}+6 \dot{\gamma} \hat{a} \eta_{0}-9 \dot{\gamma} n_{1}^{2} \eta_{0}-9 \dot{\gamma} a \hat{l} n_{1}^{2} \eta_{0}+3 \dot{\gamma} n_{1}^{4} \eta_{0}+3 \dot{\gamma} \hat{a} 1 n_{1}^{4} \eta_{0}-12 \dot{\gamma} n_{1}^{2} n_{2}^{2} \eta_{0}-12 \dot{\gamma} \hat{a} n_{1}^{2} n_{2}^{2} \eta_{0}+\right.\right.$ $8 \dot{\gamma} \hat{\beta} n_{1}^{2} n_{2}^{2} \eta_{0}+6 \dot{\gamma} n_{1}^{4} n_{2}^{2} \eta_{0}+6 \dot{\gamma} \hat{a} n_{1}^{4} n_{2}^{2} \eta_{0}-4 \dot{\gamma} \hat{\beta} n_{1}^{4} n_{2}^{2} \eta_{0}+12 \dot{\gamma} n_{1}^{2} n_{1} \eta_{0}+12 \dot{\gamma} \hat{a} n_{1}^{2} n_{1} \eta_{0}-12 \dot{\gamma} n_{1}^{4} r_{1} \eta_{0}-$ $12 \dot{\gamma} \hat{a} n_{1}^{4} r_{1} \eta_{0}-6 \dot{\gamma} n_{1}^{2} n_{2}^{2} r_{1} \eta_{0}-12 \dot{\gamma} a \hat{l} n_{1}^{2} n_{2}^{2} r_{1} \eta_{0}+4 \dot{\gamma} \hat{\beta} n_{1}^{2} n_{2}^{2} r_{1} \eta_{0}-6 \dot{\gamma} n_{1}^{4} n_{2}^{2} r_{1} \eta_{0}+4 \dot{\gamma} \hat{\beta} n_{1}^{4} n_{2}^{2} r_{1} \eta_{0}-$ $3 \dot{\gamma} n_{1}^{2} r_{2} \eta_{0}-3 \dot{\gamma} a \hat{a} n_{1}^{2} r_{2} \eta_{0}+9 \dot{\gamma} n_{1}^{4} r_{2} \eta_{0}+9 \dot{\gamma} a \hat{l} n_{1}^{4} r_{2} \eta_{0}-6 \dot{\gamma} a \hat{l} n_{1}^{4} n_{2}^{2} r_{2} \eta_{0}-3 \dot{\gamma}^{2} n_{1} n_{2} \eta_{0} \theta_{0}-$ $\left.6 \dot{\gamma}^{2} \hat{a} n_{1} n_{2} \eta_{0} \theta_{0}+2 \dot{\gamma}^{2} \hat{\beta} n_{1} n_{2} \eta_{0} \theta_{0}+9 \dot{\gamma}^{2} n_{1}^{3} n_{2} \eta_{0} \theta_{0}+12 \dot{\gamma}^{2} \hat{a} n_{1}^{3} n_{2} \eta_{0} \theta_{0}-6 \dot{\gamma}^{2} \hat{\beta} n_{1}^{3} n_{2} \eta_{0} \theta_{0}\right) /$ 
$\left(12 n_{1} n_{2}-18 n_{1}^{3} n_{2}+6 n_{1}^{5} n_{2}-12 n_{1} n_{2}^{3}+6 n_{1}^{3} n_{2}^{3}-12 n_{1} n_{2} r_{1}+42 n_{1}^{3} n_{2} r_{1}-30 n_{1}^{5} n_{2} r_{1}+30 n_{1} n_{2}^{3} r_{1}-30 n_{1}^{3} n_{2}^{3} r_{1}-\right.$ $24 n_{1}^{3} n_{2} r_{1}^{2}+24 n_{1}^{5} n_{2} r_{1}^{2}+24 n_{1}^{3} n_{2}^{3} r_{1}^{2}-6 n_{1}^{3} n_{2} r_{2}+18 n_{1}^{5} n_{2} r_{2}-12 n_{1} n_{2}^{3} r_{2}+18 n_{1}^{3} n_{2}^{3} r_{2}+6 n_{1}^{3} n_{2} r_{1} r_{2}-$ $18 n_{1}^{5} n_{2} r_{1} r_{2}-6 n_{1} n_{2}^{3} r_{1} r_{2}-18 n_{1}^{3} n_{2}^{3} r_{1} r_{2}+6 \dot{\gamma} \theta_{0}-9 \dot{\gamma} n_{1}^{2} \theta_{0}+3 \dot{\gamma} n_{1}^{4} \theta_{0}+3 \dot{\gamma} n_{2}^{2} \theta_{0}+3 \dot{\gamma} n_{1}^{2} n_{2}^{2} \theta_{0}+$ $\left.12 \dot{\gamma} n_{1}^{2} r_{1} \theta_{0}-12 \dot{\gamma} n_{1}^{4} r_{1} \theta_{0}-12 \dot{\gamma} n_{1}^{2} n_{2}^{2} r_{1} \theta_{0}-3 \dot{\gamma} n_{1}^{2} r_{2} \theta_{0}+9 \dot{\gamma} n_{1}^{4} r_{2} \theta_{0}-3 \dot{\gamma} n_{2}^{2} r_{2} \theta_{0}+9 \dot{\gamma} n_{1}^{2} n_{2}^{2} r_{2} \theta_{0}\right)-$ $\left(\left(-36 \dot{\gamma} \eta_{0}-36 \dot{\gamma}\right.\right.$ all $\eta_{0}+54 \dot{\gamma} n_{1}^{2} \eta_{0}+54 \dot{\gamma} \hat{a l} n_{1}^{2} \eta_{0}-18 \dot{\gamma} n_{1}^{4} \eta_{0}-18 \dot{\gamma} \hat{a l} n_{1}^{4} \eta_{0}+54 \dot{\gamma} n_{2}^{2} \eta_{0}+54 \dot{\gamma} \hat{a l} n_{2}^{2} \eta_{0}-$ $36 \dot{\gamma} n_{1}^{2} n_{2}^{2} \eta_{0}-72 \dot{\gamma} \hat{a} n_{1}^{2} n_{2}^{2} \eta_{0}-24 \dot{\gamma} \hat{\beta} n_{1}^{2} n_{2}^{2} \eta_{0}+72 \dot{\gamma} n_{1}^{4} n_{2}^{2} \eta_{0}+144 \dot{\gamma} a \hat{l} n_{1}^{4} n_{2}^{2} \eta_{0}-24 \dot{\gamma} \hat{\beta} n_{1}^{4} n_{2}^{2} \eta_{0}-$ $72 \dot{\gamma} n_{1}^{6} n_{2}^{2} \eta_{0}-108 \dot{\gamma} \hat{a l} n_{1}^{6} n_{2}^{2} \eta_{0}+48 \dot{\gamma} \hat{\beta} n_{1}^{6} n_{2}^{2} \eta_{0}-18 \dot{\gamma} n_{2}^{4} \eta_{0}-18 \dot{\gamma} \hat{a} 1 n_{2}^{4} \eta_{0}+72 \dot{\gamma} n_{1}^{2} n_{2}^{4} \eta_{0}+$ $144 \dot{\gamma} a 1 n_{1}^{2} n_{2}^{4} \eta_{0}-24 \dot{\gamma} \hat{\beta} n_{1}^{2} n_{2}^{4} \eta_{0}-144 \dot{\gamma} n_{1}^{4} n_{2}^{4} \eta_{0}-216 \dot{\gamma} \hat{a} n_{1}^{4} n_{2}^{4} \eta_{0}+96 \dot{\gamma} \hat{\beta} n_{1}^{4} n_{2}^{4} \eta_{0}-72 \dot{\gamma} n_{1}^{2} n_{2}^{6} \eta_{0}-$ $108 \dot{\gamma} \hat{a} n_{1}^{2} n_{2}^{6} \eta_{0}+48 \dot{\gamma} \hat{\beta} n_{1}^{2} n_{2}^{6} \eta_{0}-72 \dot{\gamma} n_{1}^{2} r_{1} \eta_{0}-72 \dot{\gamma}$ al $n_{1}^{2} r_{1} \eta_{0}+72 \dot{\gamma} n_{1}^{4} r_{1} \eta_{0}+72 \dot{\gamma} \hat{a} n_{1}^{4} r_{1} \eta_{0}-$ $72 \dot{\gamma} n_{2}^{2} r_{1} \eta_{0}-72 \dot{\gamma} \hat{a l} n_{2}^{2} r_{1} \eta_{0}+288 \dot{\gamma} n_{1}^{2} n_{2}^{2} r_{1} \eta_{0}+360 \dot{\gamma} \hat{a l} n_{1}^{2} n_{2}^{2} r_{1} \eta_{0}-48 \dot{\gamma} \hat{\beta} n_{1}^{2} n_{2}^{2} r_{1} \eta_{0}-$ $288 \dot{\gamma} n_{1}^{4} n_{2}^{2} r_{1} \eta_{0}-468 \dot{\gamma} \hat{a} n_{1}^{4} n_{2}^{2} r_{1} \eta_{0}+72 \dot{\gamma} \hat{\beta} n_{1}^{4} n_{2}^{2} r_{1} \eta_{0}+144 \dot{\gamma} n_{1}^{6} n_{2}^{2} r_{1} \eta_{0}+252 \dot{\gamma} \hat{l} n_{1}^{6} n_{2}^{2} r_{1} \eta_{0}-$ $96 \dot{\gamma} \hat{\beta} n_{1}^{6} n_{2}^{2} r_{1} \eta_{0}+72 \dot{\gamma} n_{2}^{4} r_{1} \eta_{0}+72 \dot{\gamma}$ al $n_{2}^{4} r_{1} \eta_{0}-288 \dot{\gamma} n_{1}^{2} n_{2}^{4} r_{1} \eta_{0}-468 \dot{\gamma}$ al $n_{1}^{2} n_{2}^{4} r_{1} \eta_{0}+$ $72 \dot{\gamma} \hat{\beta} n_{1}^{2} n_{2}^{4} r_{1} \eta_{0}+288 \dot{\gamma} n_{1}^{4} n_{2}^{4} r_{1} \eta_{0}+504 \dot{\gamma} \hat{a} 1 n_{1}^{4} n_{2}^{4} r_{1} \eta_{0}-192 \dot{\gamma} \hat{\beta} n_{1}^{4} n_{2}^{4} r_{1} \eta_{0}+144 \dot{\gamma} n_{1}^{2} n_{2}^{6} r_{1} \eta_{0}+$ $252 \dot{\gamma} \hat{a} 1 n_{1}^{2} n_{2}^{6} r_{1} \eta_{0}-96 \dot{\gamma} \hat{\beta} n_{1}^{2} n_{2}^{6} r_{1} \eta_{0}-144 \dot{\gamma} n_{1}^{2} n_{2}^{2} r_{1}^{2} \eta_{0}-144 \dot{\gamma} \hat{a} n_{1}^{2} n_{2}^{2} r_{1}^{2} \eta_{0}+216 \dot{\gamma} n_{1}^{4} n_{2}^{2} r_{1}^{2} \eta_{0}+$ $288 \dot{\gamma} \hat{a l} n_{1}^{4} n_{2}^{2} r_{1}^{2} \eta_{0}-48 \dot{\gamma} \hat{\beta} n_{1}^{4} n_{2}^{2} r_{1}^{2} \eta_{0}-72 \dot{\gamma} n_{1}^{6} n_{2}^{2} r_{1}^{2} \eta_{0}-144 \dot{\gamma} a \hat{l} n_{1}^{6} n_{2}^{2} r_{1}^{2} \eta_{0}+48 \dot{\gamma} \hat{\beta} n_{1}^{6} n_{2}^{2} r_{1}^{2} \eta_{0}+$ $216 \dot{\gamma} n_{1}^{2} n_{2}^{4} r_{1}^{2} \eta_{0}+288 \dot{\gamma} \hat{a} n_{1}^{2} n_{2}^{4} r_{1}^{2} \eta_{0}-48 \dot{\gamma} \hat{\beta} n_{1}^{2} n_{2}^{4} r_{1}^{2} \eta_{0}-144 \dot{\gamma} n_{1}^{4} n_{2}^{4} r_{1}^{2} \eta_{0}-288 \dot{\gamma} a \hat{l} n_{1}^{4} n_{2}^{4} r_{1}^{2} \eta_{0}+$ $96 \dot{\gamma} \hat{\beta} n_{1}^{4} n_{2}^{4} r_{1}^{2} \eta_{0}-72 \dot{\gamma} n_{1}^{2} n_{2}^{6} r_{1}^{2} \eta_{0}-144 \dot{\gamma} \hat{a} 1 n_{1}^{2} n_{2}^{6} r_{1}^{2} \eta_{0}+48 \dot{\gamma} \hat{\beta} n_{1}^{2} n_{2}^{6} r_{1}^{2} \eta_{0}+18 \dot{\gamma} n_{1}^{2} r_{2} \eta_{0}+$ $18 \dot{\gamma} \hat{a} n_{1}^{2} r_{2} \eta_{0}-54 \dot{\gamma} n_{1}^{4} r_{2} \eta_{0}-54 \dot{\gamma} \hat{a l} n_{1}^{4} r_{2} \eta_{0}+18 \dot{\gamma} n_{2}^{2} r_{2} \eta_{0}+18 \dot{\gamma}$ all $n_{2}^{2} r_{2} \eta_{0}-72 \dot{\gamma} n_{1}^{2} n_{2}^{2} r_{2} \eta_{0}-$ $72 \dot{\gamma}$ al $n_{1}^{2} n_{2}^{2} r_{2} \eta_{0}+108 \dot{\gamma} n_{1}^{4} n_{2}^{2} r_{2} \eta_{0}+144 \dot{\gamma} a 1 n_{1}^{4} n_{2}^{2} r_{2} \eta_{0}-36 \dot{\gamma}$ all $n_{1}^{6} n_{2}^{2} r_{2} \eta_{0}-54 \dot{\gamma} n_{2}^{4} r_{2} \eta_{0}-$ $54 \dot{\gamma} \hat{a} 1 n_{2}^{4} r_{2} \eta_{0}+108 \dot{\gamma} n_{1}^{2} n_{2}^{4} r_{2} \eta_{0}+144 \dot{\gamma}$ âl $n_{1}^{2} n_{2}^{4} r_{2} \eta_{0}-72 \dot{\gamma} \hat{a} 1 n_{1}^{4} n_{2}^{4} r_{2} \eta_{0}-36 \dot{\gamma}$ all $n_{1}^{2} n_{2}^{6} r_{2} \eta_{0}+$ $72 \dot{\gamma} n_{1}^{2} n_{2}^{2} r_{1} r_{2} \eta_{0}+72 \dot{\gamma} \hat{a} 1 n_{1}^{2} n_{2}^{2} r_{1} r_{2} \eta_{0}-108 \dot{\gamma} n_{1}^{4} n_{2}^{2} r_{1} r_{2} \eta_{0}-108 \dot{\gamma} \hat{a l} n_{1}^{4} n_{2}^{2} r_{1} r_{2} \eta_{0}+$ $36 \dot{\gamma} \hat{a} 1 n_{1}^{6} n_{2}^{2} r_{1} r_{2} \eta_{0}-108 \dot{\gamma} n_{1}^{2} n_{2}^{4} r_{1} r_{2} \eta_{0}-108 \dot{\gamma}$ al $n_{1}^{2} n_{2}^{4} r_{1} r_{2} \eta_{0}+72 \dot{\gamma} \hat{a l} n_{1}^{4} n_{2}^{4} r_{1} r_{2} \eta_{0}+$ $36 \dot{\gamma} \hat{a l} n_{1}^{2} n_{2}^{6} r_{1} r_{2} \eta_{0}-18 \dot{\gamma}^{2} n_{1}^{3} n_{2} \eta_{0} \theta_{0}+12 \dot{\gamma}^{2} \hat{\beta} n_{1}^{3} n_{2} \eta_{0} \theta_{0}-36 \dot{\gamma}^{2} n_{1}^{5} n_{2} \eta_{0} \theta_{0}-54 \dot{\gamma}^{2} \hat{a l} n_{1}^{5} n_{2} \eta_{0} \theta_{0}+$ $24 \dot{\gamma}^{2} \hat{\beta} n_{1}^{5} n_{2} \eta_{0} \theta_{0}+18 \dot{\gamma}^{2} n_{1} n_{2}^{3} \eta_{0} \theta_{0}-12 \dot{\gamma}^{2} \hat{\beta} n_{1} n_{2}^{3} \eta_{0} \theta_{0}+36 \dot{\gamma}^{2} n_{1} n_{2}^{5} \eta_{0} \theta_{0}+54 \dot{\gamma}^{2} \hat{a} 1 n_{1} n_{2}^{5} \eta_{0} \theta_{0}-$ $24 \dot{\gamma}^{2} \hat{\beta} n_{1} n_{2}^{5} \eta_{0} \theta_{0}-36 \dot{\gamma}^{2} n_{1}^{3} n_{2} r_{1} \eta_{0} \theta_{0}-72 \dot{\gamma}^{2} \hat{a} 1 n_{1}^{3} n_{2} r_{1} \eta_{0} \theta_{0}+24 \dot{\gamma}^{2} \hat{\beta} n_{1}^{3} n_{2} r_{1} \eta_{0} \theta_{0}+$ $36 \dot{\gamma}^{2} n_{1}^{5} n_{2} r_{1} \eta_{0} \theta_{0}+72 \dot{\gamma}^{2} \hat{a} 1 n_{1}^{5} n_{2} r_{1} \eta_{0} \theta_{0}-24 \dot{\gamma}^{2} \hat{\beta} n_{1}^{5} n_{2} r_{1} \eta_{0} \theta_{0}+36 \dot{\gamma}^{2} n_{1} n_{2}^{3} r_{1} \eta_{0} \theta_{0}+$ $72 \dot{\gamma}^{2} \hat{\mathrm{l}} \mathrm{n}_{1} \mathrm{n}_{2}^{3} \mathrm{r}_{1} \eta_{0} \theta_{0}-24 \dot{\gamma}^{2} \hat{\beta} n_{1} n_{2}^{3} r_{1} \eta_{0} \theta_{0}-36 \dot{\gamma}^{2} n_{1} n_{2}^{5} r_{1} \eta_{0} \theta_{0}-72 \dot{\gamma}^{2} \hat{a} n_{1} n_{2}^{5} r_{1} \eta_{0} \theta_{0}+$ $\left.24 \dot{\gamma}^{2} \hat{\beta} n_{1} n_{2}^{5} r_{1} \eta_{0} \theta_{0}-18 \dot{\gamma}^{2} \hat{a l} n_{1}^{5} n_{2} r_{2} \eta_{0} \theta_{0}+18 \dot{\gamma}^{2} \hat{a} n_{1} n_{2}^{5} r_{2} \eta_{0} \theta_{0}\right)$

$\left(-12 r_{1}+18 n_{1}^{2} r_{1}-6 n_{1}^{4} r_{1}+12 n_{1}^{2} n_{2}^{2} r_{1}-24 n_{1}^{2} r_{1}^{2}+24 n_{1}^{4} r_{1}^{2}+24 n_{1}^{2} n_{2}^{2} r_{1}^{2}-24 n_{1}^{2} n_{2}^{2} r_{2}+6 n_{1}^{2} r_{1} r_{2}-\right.$ $\left.\left.18 n_{1}^{4} r_{1} r_{2}-12 n_{1}^{2} n_{2}^{2} r_{1} r_{2}+6 \dot{\gamma} n_{1} n_{2} \theta_{0}-6 \dot{\gamma} n_{1} n_{2} r_{2} \theta_{0}-6 \dot{\gamma}^{2} \theta_{0}^{2}\right)\right) /$

( $\left(12 n_{1} n_{2}-18 n_{1}^{3} n_{2}+6 n_{1}^{5} n_{2}-12 n_{1} n_{2}^{3}+6 n_{1}^{3} n_{2}^{3}-12 n_{1} n_{2} r_{1}+42 n_{1}^{3} n_{2} r_{1}-30 n_{1}^{5} n_{2} r_{1}+30 n_{1} n_{2}^{3} r_{1}-\right.$ $30 n_{1}^{3} n_{2}^{3} r_{1}-24 n_{1}^{3} n_{2} r_{1}^{2}+24 n_{1}^{5} n_{2} r_{1}^{2}+24 n_{1}^{3} n_{2}^{3} r_{1}^{2}-6 n_{1}^{3} n_{2} r_{2}+18 n_{1}^{5} n_{2} r_{2}-12 n_{1} n_{2}^{3} r_{2}+18 n_{1}^{3} n_{2}^{3} r_{2}+$ $6 n_{1}^{3} n_{2} r_{1} r_{2}-18 n_{1}^{5} n_{2} r_{1} r_{2}-6 n_{1} n_{2}^{3} r_{1} r_{2}-18 n_{1}^{3} n_{2}^{3} r_{1} r_{2}+6 \dot{\gamma} \theta_{0}-9 \dot{\gamma} n_{1}^{2} \theta_{0}+3 \dot{\gamma} n_{1}^{4} \theta_{0}+3 \dot{\gamma} n_{2}^{2} \theta_{0}+$ $3 \dot{\gamma} n_{1}^{2} n_{2}^{2} \theta_{0}+12 \dot{\gamma} n_{1}^{2} r_{1} \theta_{0}-12 \dot{\gamma} n_{1}^{4} r_{1} \theta_{0}-12 \dot{\gamma} n_{1}^{2} n_{2}^{2} r_{1} \theta_{0}-3 \dot{\gamma} n_{1}^{2} r_{2} \theta_{0}+9 \dot{\gamma} n_{1}^{4} r_{2} \theta_{0}-3 \dot{\gamma} n_{2}^{2} r_{2} \theta_{0}+$ $\left.9 \dot{\gamma} n_{1}^{2} n_{2}^{2} r_{2} \theta_{0}\right)\left(-72 n_{1}^{2} n_{2}^{2}+144 n_{1}^{4} n_{2}^{2}-72 n_{1}^{6} n_{2}^{2}+144 n_{1}^{2} n_{2}^{4}-144 n_{1}^{4} n_{2}^{4}-72 n_{1}^{2} n_{2}^{6}+72 r_{1}-108 n_{1}^{2} r_{1}+\right.$ $36 n_{1}^{4} r_{1}-108 n_{2}^{2} r_{1}+288 n_{1}^{2} n_{2}^{2} r_{1}-648 n_{1}^{4} n_{2}^{2} r_{1}+432 n_{1}^{6} n_{2}^{2} r_{1}+36 n_{2}^{4} r_{1}-648 n_{1}^{2} n_{2}^{4} r_{1}+864 n_{1}^{4} n_{2}^{4} r_{1}+$ $432 n_{1}^{2} n_{2}^{6} r_{1}+144 n_{1}^{2} r_{1}^{2}-144 n_{1}^{4} r_{1}^{2}+144 n_{2}^{2} r_{1}^{2}-720 n_{1}^{2} n_{2}^{2} r_{1}^{2}+1080 n_{1}^{4} n_{2}^{2} r_{1}^{2}-648 n_{1}^{6} n_{2}^{2} r_{1}^{2}-144 n_{2}^{4} r_{1}^{2}+$ $1080 n_{1}^{2} n_{2}^{4} r_{1}^{2}-1296 n_{1}^{4} n_{2}^{4} r_{1}^{2}-648 n_{1}^{2} n_{2}^{6} r_{1}^{2}+288 n_{1}^{2} n_{2}^{2} r_{1}^{3}-576 n_{1}^{4} n_{2}^{2} r_{1}^{3}+288 n_{1}^{6} n_{2}^{2} r_{1}^{3}-576 n_{1}^{2} n_{2}^{4} r_{1}^{3}+$ $576 n_{1}^{4} n_{2}^{4} r_{1}^{3}+288 n_{1}^{2} n_{2}^{6} r_{1}^{3}+72 n_{1}^{2} n_{2}^{2} r_{2}+144 n_{1}^{4} n_{2}^{2} r_{2}-216 n_{1}^{6} n_{2}^{2} r_{2}+144 n_{1}^{2} n_{2}^{4} r_{2}-432 n_{1}^{4} n_{2}^{4} r_{2}-$ $216 n_{1}^{2} n_{2}^{6} r_{2}-36 n_{1}^{2} r_{1} r_{2}+108 n_{1}^{4} r_{1} r_{2}-36 n_{2}^{2} r_{1} r_{2}+288 n_{1}^{2} n_{2}^{2} r_{1} r_{2}-504 n_{1}^{4} n_{2}^{2} r_{1} r_{2}+432 n_{1}^{6} n_{2}^{2} r_{1} r_{2}+$ $108 n_{2}^{4} r_{1} r_{2}-504 n_{1}^{2} n_{2}^{4} r_{1} r_{2}+864 n_{1}^{4} n_{2}^{4} r_{1} r_{2}+432 n_{1}^{2} n_{2}^{6} r_{1} r_{2}-144 n_{1}^{2} n_{2}^{2} r_{1}^{2} r_{2}+360 n_{1}^{4} n_{2}^{2} r_{1}^{2} r_{2}-$ $216 n_{1}^{6} n_{2}^{2} r_{1}^{2} r_{2}+360 n_{1}^{2} n_{2}^{4} r_{1}^{2} r_{2}-432 n_{1}^{4} n_{2}^{4} r_{1}^{2} r_{2}-216 n_{1}^{2} n_{2}^{6} r_{1}^{2} r_{2}+72 \dot{\gamma}^{2} \theta_{0}^{2}-36 \dot{\gamma}^{2} n_{1}^{2} \theta_{0}^{2}+18 \dot{\gamma}^{2} n_{1}^{4} \theta_{0}^{2}-$ $36 \dot{\gamma}^{2} n_{2}^{2} \theta_{0}^{2}+36 \dot{\gamma}^{2} n_{1}^{2} n_{2}^{2} \theta_{0}^{2}+18 \dot{\gamma}^{2} n_{2}^{4} \theta_{0}^{2}+72 \dot{\gamma}^{2} n_{1}^{2} r_{1} \theta_{0}^{2}-72 \dot{\gamma}^{2} n_{1}^{4} r_{1} \theta_{0}^{2}+72 \dot{\gamma}^{2} n_{2}^{2} r_{1} \theta_{0}^{2}-$

$144 \dot{\gamma}^{2} n_{1}^{2} n_{2}^{2} r_{1} \theta_{0}^{2}-72 \dot{\gamma}^{2} n_{2}^{4} r_{1} \theta_{0}^{2}-36 \dot{\gamma}^{2} n_{1}^{2} r_{2} \theta_{0}^{2}+54 \dot{\gamma}^{2} n_{1}^{4} r_{2} \theta_{0}^{2}-36 \dot{\gamma}^{2} n_{2}^{2} r_{2} \theta_{0}^{2}+108 \dot{\gamma}^{2} n_{1}^{2} n_{2}^{2} r_{2} \theta_{0}^{2}+$ $\left.\left.\left.54 \dot{\gamma}^{2} n_{2}^{4} r_{2} \theta_{0}^{2}\right)\right)\right)\left(/\left(-2 n_{1} n_{2}+n_{1}^{3} n_{2}+2 n_{1} n_{2} r_{1}-4 n_{1}^{3} n_{2} r_{1}+3 n_{1}^{3} n_{2} r_{2}+\dot{\gamma} \theta_{0}\right)\right.$ ， 
$-\dot{\gamma} \eta_{0}-\dot{\gamma} \hat{\mathrm{l}} \eta_{0}+3 \dot{\gamma} n_{1}^{2} n_{2}^{2} \eta_{0}+4 \dot{\gamma} \hat{\mathrm{l}} n_{1}^{2} n_{2}^{2} \eta_{0}-2 \dot{\gamma} \hat{\beta} n_{1}^{2} n_{2}^{2} \eta_{0}+$

$-2 n_{1} n_{2}+n_{1}^{3} n_{2}+2 n_{1} n_{2} r_{1}-4 n_{1}^{3} n_{2} r_{1}+3 n_{1}^{3} n_{2} r_{2}+\dot{\gamma} \theta_{0}$

$\left(6 \dot{\gamma} \eta_{0}+6 \dot{\gamma} \hat{a} \eta_{0}-9 \dot{\gamma} n_{1}^{2} \eta_{0}-9 \dot{\gamma} \hat{a} n_{1}^{2} \eta_{0}+3 \dot{\gamma} n_{1}^{4} \eta_{0}+3 \dot{\gamma} \hat{a} 1 n_{1}^{4} \eta_{0}-12 \dot{\gamma} n_{1}^{2} n_{2}^{2} \eta_{0}-12 \dot{\gamma} a \hat{l} n_{1}^{2} n_{2}^{2} \eta_{0}+\right.$ $8 \dot{\gamma} \hat{\beta} n_{1}^{2} n_{2}^{2} \eta_{0}+6 \dot{\gamma} n_{1}^{4} n_{2}^{2} \eta_{0}+6 \dot{\gamma} \hat{a} \hat{l} n_{1}^{4} n_{2}^{2} \eta_{0}-4 \dot{\gamma} \hat{\beta} n_{1}^{4} n_{2}^{2} \eta_{0}+12 \dot{\gamma} n_{1}^{2} r_{1} \eta_{0}+12 \dot{\gamma} \hat{a} n_{1}^{2} r_{1} \eta_{0}-12 \dot{\gamma} n_{1}^{4} r_{1} \eta_{0}-$ $12 \dot{\gamma} \hat{a l} n_{1}^{4} r_{1} \eta_{0}-6 \dot{\gamma} n_{1}^{2} n_{2}^{2} r_{1} \eta_{0}-12 \dot{\gamma} a \hat{l} n_{1}^{2} n_{2}^{2} r_{1} \eta_{0}+4 \dot{\gamma} \hat{\beta} n_{1}^{2} n_{2}^{2} r_{1} \eta_{0}-6 \dot{\gamma} n_{1}^{4} n_{2}^{2} r_{1} \eta_{0}+4 \dot{\gamma} \hat{\beta} n_{1}^{4} n_{2}^{2} r_{1} \eta_{0}-$ $3 \dot{\gamma} n_{1}^{2} r_{2} \eta_{0}-3 \dot{\gamma} \hat{a} n_{1}^{2} r_{2} \eta_{0}+9 \dot{\gamma} n_{1}^{4} r_{2} \eta_{0}+9 \dot{\gamma}$ âl $n_{1}^{4} r_{2} \eta_{0}-6 \dot{\gamma}$ all $n_{1}^{4} n_{2}^{2} r_{2} \eta_{0}-3 \dot{\gamma}^{2} n_{1} n_{2} \eta_{0} \theta_{0}-$ $\left.6 \dot{\gamma}^{2} \hat{a} n_{1} n_{2} \eta_{0} \theta_{0}+2 \dot{\gamma}^{2} \hat{\beta} n_{1} n_{2} \eta_{0} \theta_{0}+9 \dot{\gamma}^{2} n_{1}^{3} n_{2} \eta_{0} \theta_{0}+12 \dot{\gamma}^{2} a \hat{l} n_{1}^{3} n_{2} \eta_{0} \theta_{0}-6 \dot{\gamma}^{2} \hat{\beta} n_{1}^{3} n_{2} \eta_{0} \theta_{0}\right) /$ $\left(12 n_{1} n_{2}-18 n_{1}^{3} n_{2}+6 n_{1}^{5} n_{2}-12 n_{1} n_{2}^{3}+6 n_{1}^{3} n_{2}^{3}-12 n_{1} n_{2} n_{1}+42 n_{1}^{3} n_{2} n_{1}-30 n_{1}^{5} n_{2} r_{1}+30 n_{1} n_{2}^{3} n_{1}-\right.$ $30 n_{1}^{3} n_{2}^{3} r_{1}-24 n_{1}^{3} n_{2} r_{1}^{2}+24 n_{1}^{5} n_{2} r_{1}^{2}+24 n_{1}^{3} n_{2}^{3} r_{1}^{2}-6 n_{1}^{3} n_{2} r_{2}+18 n_{1}^{5} n_{2} r_{2}-12 n_{1} n_{2}^{3} r_{2}+18 n_{1}^{3} n_{2}^{3} r_{2}+$ $6 n_{1}^{3} n_{2} r_{1} r_{2}-18 n_{1}^{5} n_{2} r_{1} r_{2}-6 n_{1} n_{2}^{3} r_{1} r_{2}-18 n_{1}^{3} n_{2}^{3} r_{1} r_{2}+6 \dot{\gamma} \theta_{0}-9 \dot{\gamma} n_{1}^{2} \theta_{0}+3 \dot{\gamma} n_{1}^{4} \theta_{0}+3 \dot{\gamma} n_{2}^{2} \theta_{0}+$ $\left.3 \dot{\gamma} n_{1}^{2} n_{2}^{2} \theta_{0}+12 \dot{\gamma} n_{1}^{2} r_{1} \theta_{0}-12 \dot{\gamma} n_{1}^{4} r_{1} \theta_{0}-12 \dot{\gamma} n_{1}^{2} n_{2}^{2} r_{1} \theta_{0}-3 \dot{\gamma} n_{1}^{2} r_{2} \theta_{0}+9 \dot{\gamma} n_{1}^{4} r_{2} \theta_{0}-3 \dot{\gamma} n_{2}^{2} r_{2} \theta_{0}+9 \dot{\gamma} n_{1}^{2} n_{2}^{2} r_{2} \theta_{0}\right)-$ $\left(\left(2 n_{1}^{2} n_{2}^{2}+2 r_{1}-8 n_{1}^{2} n_{2}^{2} r_{1}+6 n_{1}^{2} n_{2}^{2} r_{2}\right)\right.$

$\left(-36 \dot{\gamma} \eta_{0}-36 \dot{\gamma} \hat{a} \hat{l} \eta_{0}+54 \dot{\gamma} n_{1}^{2} \eta_{0}+54 \dot{\gamma}\right.$ âl $n_{1}^{2} \eta_{0}-18 \dot{\gamma} n_{1}^{4} \eta_{0}-18 \dot{\gamma}$ âl $n_{1}^{4} \eta_{0}+54 \dot{\gamma} n_{2}^{2} \eta_{0}+54 \dot{\gamma} \hat{a} n_{2}^{2} \eta_{0}-$ $36 \dot{\gamma} n_{1}^{2} n_{2}^{2} \eta_{0}-72 \dot{\gamma} a 1 n_{1}^{2} n_{2}^{2} \eta_{0}-24 \dot{\gamma} \hat{\beta} n_{1}^{2} n_{2}^{2} \eta_{0}+72 \dot{\gamma} n_{1}^{4} n_{2}^{2} \eta_{0}+144 \dot{\gamma} a \hat{l} n_{1}^{4} n_{2}^{2} \eta_{0}-24 \dot{\gamma} \hat{\beta} n_{1}^{4} n_{2}^{2} \eta_{0}-$ $72 \dot{\gamma} n_{1}^{6} n_{2}^{2} \eta_{0}-108 \dot{\gamma} \hat{a} 1 n_{1}^{6} n_{2}^{2} \eta_{0}+48 \dot{\gamma} \hat{\beta} n_{1}^{6} n_{2}^{2} \eta_{0}-18 \dot{\gamma} n_{2}^{4} \eta_{0}-18 \dot{\gamma} \hat{a} 1 n_{2}^{4} \eta_{0}+72 \dot{\gamma} n_{1}^{2} n_{2}^{4} \eta_{0}+$ $144 \dot{\gamma} a \hat{l} n_{1}^{2} n_{2}^{4} \eta_{0}-24 \dot{\gamma} \hat{\beta} n_{1}^{2} n_{2}^{4} \eta_{0}-144 \dot{\gamma} n_{1}^{4} n_{2}^{4} \eta_{0}-216 \dot{\gamma}$ al $n_{1}^{4} n_{2}^{4} \eta_{0}+96 \dot{\gamma} \hat{\beta} n_{1}^{4} n_{2}^{4} \eta_{0}-72 \dot{\gamma} n_{1}^{2} n_{2}^{6} \eta_{0}-$ $108 \dot{\gamma} a 1 n_{1}^{2} n_{2}^{6} \eta_{0}+48 \dot{\gamma} \hat{\beta} n_{1}^{2} n_{2}^{6} \eta_{0}-72 \dot{\gamma} n_{1}^{2} r_{1} \eta_{0}-72 \dot{\gamma}$ al $n_{1}^{2} r_{1} \eta_{0}+72 \dot{\gamma} n_{1}^{4} r_{1} \eta_{0}+72 \dot{\gamma}$ al $n_{1}^{4} r_{1} \eta_{0}-$ $72 \dot{\gamma} n_{2}^{2} r_{1} \eta_{0}-72 \dot{\gamma} a \hat{l} n_{2}^{2} r_{1} \eta_{0}+288 \dot{\gamma} n_{1}^{2} n_{2}^{2} r_{1} \eta_{0}+360 \dot{\gamma}$ all $n_{1}^{2} n_{2}^{2} r_{1} \eta_{0}-48 \dot{\gamma} \hat{\beta} n_{1}^{2} n_{2}^{2} r_{1} \eta_{0}-$ $288 \dot{\gamma} n_{1}^{4} n_{2}^{2} r_{1} \eta_{0}-468 \dot{\gamma}$ al $n_{1}^{4} n_{2}^{2} r_{1} \eta_{0}+72 \dot{\gamma} \hat{\beta} n_{1}^{4} n_{2}^{2} r_{1} \eta_{0}+144 \dot{\gamma} n_{1}^{6} n_{2}^{2} r_{1} \eta_{0}+252 \dot{\gamma}$ â $n_{1}^{6} n_{2}^{2} r_{1} \eta_{0}-$ $96 \dot{\gamma} \hat{\beta} n_{1}^{6} n_{2}^{2} r_{1} \eta_{0}+72 \dot{\gamma} n_{2}^{4} r_{1} \eta_{0}+72 \dot{\gamma}$ al $n_{2}^{4} r_{1} \eta_{0}-288 \dot{\gamma} n_{1}^{2} n_{2}^{4} r_{1} \eta_{0}-468 \dot{\gamma}$ al $n_{1}^{2} n_{2}^{4} r_{1} \eta_{0}+$ $72 \dot{\gamma} \hat{\beta} n_{1}^{2} n_{2}^{4} r_{1} \eta_{0}+288 \dot{\gamma} n_{1}^{4} n_{2}^{4} r_{1} \eta_{0}+504 \dot{\gamma} a 1 n_{1}^{4} n_{2}^{4} r_{1} \eta_{0}-192 \dot{\gamma} \hat{\beta} n_{1}^{4} n_{2}^{4} r_{1} \eta_{0}+144 \dot{\gamma} n_{1}^{2} n_{2}^{6} r_{1} \eta_{0}+$ $252 \dot{\gamma} \hat{a} 1 n_{1}^{2} n_{2}^{6} r_{1} \eta_{0}-96 \dot{\gamma} \hat{\beta} n_{1}^{2} n_{2}^{6} r_{1} \eta_{0}-144 \dot{\gamma} n_{1}^{2} n_{2}^{2} r_{1}^{2} \eta_{0}-144 \dot{\gamma}$ al $n_{1}^{2} n_{2}^{2} r_{1}^{2} \eta_{0}+216 \dot{\gamma} n_{1}^{4} n_{2}^{2} r_{1}^{2} \eta_{0}+$ $288 \dot{\gamma} \hat{a l} n_{1}^{4} n_{2}^{2} r_{1}^{2} \eta_{0}-48 \dot{\gamma} \hat{\beta} n_{1}^{4} n_{2}^{2} r_{1}^{2} \eta_{0}-72 \dot{\gamma} n_{1}^{6} n_{2}^{2} r_{1}^{2} \eta_{0}-144 \dot{\gamma}$ al $n_{1}^{6} n_{2}^{2} r_{1}^{2} \eta_{0}+48 \dot{\gamma} \hat{\beta} n_{1}^{6} n_{2}^{2} r_{1}^{2} \eta_{0}+$ $216 \dot{\gamma} n_{1}^{2} n_{2}^{4} r_{1}^{2} \eta_{0}+288 \dot{\gamma}$ al $n_{1}^{2} n_{2}^{4} r_{1}^{2} \eta_{0}-48 \dot{\gamma} \hat{\beta} n_{1}^{2} n_{2}^{4} r_{1}^{2} \eta_{0}-144 \dot{\gamma} n_{1}^{4} n_{2}^{4} r_{1}^{2} \eta_{0}-288 \dot{\gamma}$ al $n_{1}^{4} n_{2}^{4} r_{1}^{2} \eta_{0}+$ $96 \dot{\gamma} \hat{\beta} n_{1}^{4} n_{2}^{4} r_{1}^{2} \eta_{0}-72 \dot{\gamma} n_{1}^{2} n_{2}^{6} r_{1}^{2} \eta_{0}-144 \dot{\gamma} \hat{a l} n_{1}^{2} n_{2}^{6} r_{1}^{2} \eta_{0}+48 \dot{\gamma} \hat{\beta} n_{1}^{2} n_{2}^{6} r_{1}^{2} \eta_{0}+18 \dot{\gamma} n_{1}^{2} r_{2} \eta_{0}+$ $18 \dot{\gamma}$ al $n_{1}^{2} r_{2} \eta_{0}-54 \dot{\gamma} n_{1}^{4} r_{2} \eta_{0}-54 \dot{\gamma}$ al $n_{1}^{4} r_{2} \eta_{0}+18 \dot{\gamma} n_{2}^{2} r_{2} \eta_{0}+18 \dot{\gamma}$ all $n_{2}^{2} r_{2} \eta_{0}-72 \dot{\gamma} n_{1}^{2} n_{2}^{2} r_{2} \eta_{0}-$ $72 \dot{\gamma} a 1 n_{1}^{2} n_{2}^{2} r_{2} \eta_{0}+108 \dot{\gamma} n_{1}^{4} n_{2}^{2} r_{2} \eta_{0}+144 \dot{\gamma}$ all $n_{1}^{4} n_{2}^{2} r_{2} \eta_{0}-36 \dot{\gamma}$ al $n_{1}^{6} n_{2}^{2} r_{2} \eta_{0}-54 \dot{\gamma} n_{2}^{4} r_{2} \eta_{0}-$ $54 \dot{\gamma}$ al $n_{2}^{4} r_{2} \eta_{0}+108 \dot{\gamma} n_{1}^{2} n_{2}^{4} r_{2} \eta_{0}+144 \dot{\gamma}$ al $n_{1}^{2} n_{2}^{4} r_{2} \eta_{0}-72 \dot{\gamma}$ all $n_{1}^{4} n_{2}^{4} r_{2} \eta_{0}-36 \dot{\gamma}$ all $n_{1}^{2} n_{2}^{6} r_{2} \eta_{0}+$ $72 \dot{\gamma} n_{1}^{2} n_{2}^{2} r_{1} r_{2} \eta_{0}+72 \dot{\gamma}$ al $n_{1}^{2} n_{2}^{2} r_{1} r_{2} \eta_{0}-108 \dot{\gamma} n_{1}^{4} n_{2}^{2} r_{1} r_{2} \eta_{0}-108 \dot{\gamma} \hat{a} n_{1}^{4} n_{2}^{2} r_{1} r_{2} \eta_{0}+$ $36 \dot{\gamma} a \hat{l} n_{1}^{6} n_{2}^{2} r_{1} r_{2} \eta_{0}-108 \dot{\gamma} n_{1}^{2} n_{2}^{4} r_{1} r_{2} \eta_{0}-108 \dot{\gamma} a 1 n_{1}^{2} n_{2}^{4} r_{1} r_{2} \eta_{0}+72 \dot{\gamma} a 1 n_{1}^{4} n_{2}^{4} r_{1} r_{2} \eta_{0}+$ $36 \dot{\gamma} \hat{a} 1 n_{1}^{2} n_{2}^{6} r_{1} r_{2} \eta_{0}-18 \dot{\gamma}^{2} n_{1}^{3} n_{2} \eta_{0} \theta_{0}+12 \dot{\gamma}^{2} \hat{\beta} n_{1}^{3} n_{2} \eta_{0} \theta_{0}-36 \dot{\gamma}^{2} n_{1}^{5} n_{2} \eta_{0} \theta_{0}-54 \dot{\gamma}^{2} \hat{a l} n_{1}^{5} n_{2} \eta_{0} \theta_{0}+$ $24 \dot{\gamma}^{2} \hat{\beta} n_{1}^{5} n_{2} \eta_{0} \theta_{0}+18 \dot{\gamma}^{2} n_{1} n_{2}^{3} \eta_{0} \theta_{0}-12 \dot{\gamma}^{2} \hat{\beta} n_{1} n_{2}^{3} \eta_{0} \theta_{0}+36 \dot{\gamma}^{2} n_{1} n_{2}^{5} \eta_{0} \theta_{0}+54 \dot{\gamma}^{2} \hat{a} 1 n_{1} n_{2}^{5} \eta_{0} \theta_{0}-$ $24 \dot{\gamma}^{2} \hat{\beta} n_{1} n_{2}^{5} \eta_{0} \theta_{0}-36 \dot{\gamma}^{2} n_{1}^{3} n_{2} r_{1} \eta_{0} \theta_{0}-72 \dot{\gamma}^{2} \hat{a} 1 n_{1}^{3} n_{2} r_{1} \eta_{0} \theta_{0}+24 \dot{\gamma}^{2} \hat{\beta} n_{1}^{3} n_{2} r_{1} \eta_{0} \theta_{0}+36 \dot{\gamma}^{2} n_{1}^{5} n_{2} r_{1} \eta_{0} \theta_{0}+$ $72 \dot{\gamma}^{2} \hat{1} n_{1}^{5} n_{2} r_{1} \eta_{0} \theta_{0}-24 \dot{\gamma}^{2} \hat{\beta} n_{1}^{5} n_{2} r_{1} \eta_{0} \theta_{0}+36 \dot{\gamma}^{2} n_{1} n_{2}^{3} r_{1} \eta_{0} \theta_{0}+72 \dot{\gamma}^{2} \hat{a} n_{1} n_{2}^{3} r_{1} \eta_{0} \theta_{0}-$ $24 \dot{\gamma}^{2} \hat{\beta} n_{1} n_{2}^{3} r_{1} \eta_{0} \theta_{0}-36 \dot{\gamma}^{2} n_{1} n_{2}^{5} r_{1} \eta_{0} \theta_{0}-72 \dot{\gamma}^{2} a \hat{l} n_{1} n_{2}^{5} r_{1} \eta_{0} \theta_{0}+24 \dot{\gamma}^{2} \hat{\beta} n_{1} n_{2}^{5} r_{1} \eta_{0} \theta_{0}-$ $\left.\left.18 \dot{\gamma}^{2} \hat{a} n_{1}^{5} n_{2} r_{2} \eta_{0} \theta_{0}+18 \dot{r}^{2} \hat{a l} n_{1} n_{2}^{5} r_{2} \eta_{0} \theta_{0}\right)\right) /$

$\left(\left(-2 n_{1} n_{2}+n_{1}^{3} n_{2}+2 n_{1} n_{2} r_{1}-4 n_{1}^{3} n_{2} r_{1}+3 n_{1}^{3} n_{2} r_{2}+\dot{\gamma} \theta_{0}\right)\right.$

$\left(-72 n_{1}^{2} n_{2}^{2}+144 n_{1}^{4} n_{2}^{2}-72 n_{1}^{6} n_{2}^{2}+144 n_{1}^{2} n_{2}^{4}-144 n_{1}^{4} n_{2}^{4}-72 n_{1}^{2} n_{2}^{6}+72 r_{1}-108 n_{1}^{2} r_{1}+36 n_{1}^{4} r_{1}-108 n_{2}^{2} r_{1}+\right.$ $288 n_{1}^{2} n_{2}^{2} r_{1}-648 n_{1}^{4} n_{2}^{2} r_{1}+432 n_{1}^{6} n_{2}^{2} r_{1}+36 n_{2}^{4} r_{1}-648 n_{1}^{2} n_{2}^{4} r_{1}+864 n_{1}^{4} n_{2}^{4} r_{1}+432 n_{1}^{2} n_{2}^{6} r_{1}+144 n_{1}^{2} r_{1}^{2}-$ $144 n_{1}^{4} r_{1}^{2}+144 n_{2}^{2} r_{1}^{2}-720 n_{1}^{2} n_{2}^{2} r_{1}^{2}+1080 n_{1}^{4} n_{2}^{2} r_{1}^{2}-648 n_{1}^{6} n_{2}^{2} r_{1}^{2}-144 n_{2}^{4} r_{1}^{2}+1080 n_{1}^{2} n_{2}^{4} r_{1}^{2}-1296 n_{1}^{4} n_{2}^{4} r_{1}^{2}-$ $648 n_{1}^{2} n_{2}^{6} r_{1}^{2}+288 n_{1}^{2} n_{2}^{2} r_{1}^{3}-576 n_{1}^{4} n_{2}^{2} r_{1}^{3}+288 n_{1}^{6} n_{2}^{2} r_{1}^{3}-576 n_{1}^{2} n_{2}^{4} r_{1}^{3}+576 n_{1}^{4} n_{2}^{4} r_{1}^{3}+288 n_{1}^{2} n_{2}^{6} r_{1}^{3}+$ $72 n_{1}^{2} n_{2}^{2} r_{2}+144 n_{1}^{4} n_{2}^{2} r_{2}-216 n_{1}^{6} n_{2}^{2} r_{2}+144 n_{1}^{2} n_{2}^{4} r_{2}-432 n_{1}^{4} n_{2}^{4} r_{2}-216 n_{1}^{2} n_{2}^{6} r_{2}-36 n_{1}^{2} r_{1} r_{2}+$ $108 n_{1}^{4} r_{1} r_{2}-36 n_{2}^{2} r_{1} r_{2}+288 n_{1}^{2} n_{2}^{2} r_{1} r_{2}-504 n_{1}^{4} n_{2}^{2} r_{1} r_{2}+432 n_{1}^{6} n_{2}^{2} r_{1} r_{2}+108 n_{2}^{4} r_{1} r_{2}-504 n_{1}^{2} n_{2}^{4} r_{1} r_{2}+$ $864 n_{1}^{4} n_{2}^{4} r_{1} r_{2}+432 n_{1}^{2} n_{2}^{6} r_{1} r_{2}-144 n_{1}^{2} n_{2}^{2} r_{1}^{2} r_{2}+360 n_{1}^{4} n_{2}^{2} r_{1}^{2} r_{2}-216 n_{1}^{6} n_{2}^{2} r_{1}^{2} r_{2}+360 n_{1}^{2} n_{2}^{4} r_{1}^{2} r_{2}-$ $432 n_{1}^{4} n_{2}^{4} r_{1}^{2} r_{2}-216 n_{1}^{2} n_{2}^{6} r_{1}^{2} r_{2}+72 \dot{\gamma}^{2} \theta_{0}^{2}-36 \dot{\gamma}^{2} n_{1}^{2} \theta_{0}^{2}+18 \dot{\gamma}^{2} n_{1}^{4} \theta_{0}^{2}-36 \dot{\gamma}^{2} n_{2}^{2} \theta_{0}^{2}+36 \dot{\gamma}^{2} n_{1}^{2} n_{2}^{2} \theta_{0}^{2}+$ $18 \dot{\gamma}^{2} n_{2}^{4} \theta_{0}^{2}+72 \dot{\gamma}^{2} n_{1}^{2} r_{1} \theta_{0}^{2}-72 \dot{\gamma}^{2} n_{1}^{4} r_{1} \theta_{0}^{2}+72 \dot{\gamma}^{2} n_{2}^{2} r_{1} \theta_{0}^{2}-144 \dot{\gamma}^{2} n_{1}^{2} n_{2}^{2} r_{1} \theta_{0}^{2}-72 \dot{\gamma}^{2} n_{2}^{4} r_{1} \theta_{0}^{2}-$ $\left.\left.36 \dot{\gamma}^{2} n_{1}^{2} r_{2} \theta_{0}^{2}+54 \dot{\gamma}^{2} n_{1}^{4} r_{2} \theta_{0}^{2}-36 \dot{\gamma}^{2} n_{2}^{2} r_{2} \theta_{0}^{2}+108 \dot{\gamma}^{2} n_{1}^{2} n_{2}^{2} r_{2} \theta_{0}^{2}+54 \dot{\gamma}^{2} n_{2}^{4} r_{2} \theta_{0}^{2}\right)\right)-$ 
$\left(\left(-36 \dot{\gamma} \eta_{0}-36 \dot{\gamma}\right.\right.$ all $\eta_{0}+54 \dot{\gamma} n_{1}^{2} \eta_{0}+54 \dot{\gamma}$ âl $n_{1}^{2} \eta_{0}-18 \dot{\gamma} n_{1}^{4} \eta_{0}-18 \dot{\gamma}$ âl $n_{1}^{4} \eta_{0}+54 \dot{\gamma} n_{2}^{2} \eta_{0}+54 \dot{\gamma}$ all $n_{2}^{2} \eta_{0}-$ $36 \dot{\gamma} n_{1}^{2} n_{2}^{2} \eta_{0}-72 \dot{\gamma}$ al $n_{1}^{2} n_{2}^{2} \eta_{0}-24 \dot{\gamma} \hat{\beta} n_{1}^{2} n_{2}^{2} \eta_{0}+72 \dot{\gamma} n_{1}^{4} n_{2}^{2} \eta_{0}+144 \dot{\gamma}$ al $n_{1}^{4} n_{2}^{2} \eta_{0}-24 \dot{\gamma} \hat{\beta} n_{1}^{4} n_{2}^{2} \eta_{0}-$ $72 \dot{\gamma} n_{1}^{6} n_{2}^{2} \eta_{0}-108 \dot{\gamma} \hat{a} n_{1}^{6} n_{2}^{2} \eta_{0}+48 \dot{\gamma} \hat{\beta} n_{1}^{6} n_{2}^{2} \eta_{0}-18 \dot{\gamma} n_{2}^{4} \eta_{0}-18 \dot{\gamma} \hat{a} n_{2}^{4} \eta_{0}+72 \dot{\gamma} n_{1}^{2} n_{2}^{4} \eta_{0}+144 \dot{\gamma} a \hat{l} n_{1}^{2} n_{2}^{4} \eta_{0}-$ $24 \dot{\gamma} \hat{\beta} n_{1}^{2} n_{2}^{4} \eta_{0}-144 \dot{\gamma} n_{1}^{4} n_{2}^{4} \eta_{0}-216 \dot{\gamma}$ al $n_{1}^{4} n_{2}^{4} \eta_{0}+96 \dot{\gamma} \hat{\beta} n_{1}^{4} n_{2}^{4} \eta_{0}-72 \dot{\gamma} n_{1}^{2} n_{2}^{6} \eta_{0}-108 \dot{\gamma} \hat{a} n_{1}^{2} n_{2}^{6} \eta_{0}+$ $48 \dot{\gamma} \hat{\beta} n_{1}^{2} n_{2}^{6} \eta_{0}-72 \dot{\gamma} n_{1}^{2} r_{1} \eta_{0}-72 \dot{\gamma} \hat{a l} n_{1}^{2} r_{1} \eta_{0}+72 \dot{\gamma} n_{1}^{4} r_{1} \eta_{0}+72 \dot{\gamma}$ âl $n_{1}^{4} r_{1} \eta_{0}-72 \dot{\gamma} n_{2}^{2} r_{1} \eta_{0}-$ $72 \dot{\gamma} a \hat{l} n_{2}^{2} n_{1} \eta_{0}+288 \dot{\gamma} n_{1}^{2} n_{2}^{2} n_{1} \eta_{0}+360 \dot{\gamma}$ al $n_{1}^{2} n_{2}^{2} n_{1} \eta_{0}-48 \dot{\gamma} \hat{\beta} n_{1}^{2} n_{2}^{2} n_{1} \eta_{0}-288 \dot{\gamma} n_{1}^{4} n_{2}^{2} n_{1} \eta_{0}-$ $468 \dot{\gamma} \hat{a} 1 n_{1}^{4} n_{2}^{2} r_{1} \eta_{0}+72 \dot{\gamma} \hat{\beta} n_{1}^{4} n_{2}^{2} r_{1} \eta_{0}+144 \dot{\gamma} n_{1}^{6} n_{2}^{2} r_{1} \eta_{0}+252 \dot{\gamma}$ al $n_{1}^{6} n_{2}^{2} r_{1} \eta_{0}-96 \dot{\gamma} \hat{\beta} n_{1}^{6} n_{2}^{2} r_{1} \eta_{0}+$ $72 \dot{\gamma} n_{2}^{4} r_{1} \eta_{0}+72 \dot{\gamma} \hat{a l} n_{2}^{4} r_{1} \eta_{0}-288 \dot{\gamma} n_{1}^{2} n_{2}^{4} r_{1} \eta_{0}-468 \dot{\gamma} \hat{a} n_{1}^{2} n_{2}^{4} r_{1} \eta_{0}+72 \dot{\gamma} \hat{\beta} n_{1}^{2} n_{2}^{4} r_{1} \eta_{0}+288 \dot{\gamma} n_{1}^{4} n_{2}^{4} r_{1} \eta_{0}+$ $504 \dot{\gamma} \hat{a} 1 n_{1}^{4} n_{2}^{4} r_{1} \eta_{0}-192 \dot{\gamma} \hat{\beta} n_{1}^{4} n_{2}^{4} r_{1} \eta_{0}+144 \dot{\gamma} n_{1}^{2} n_{2}^{6} r_{1} \eta_{0}+252 \dot{\gamma} \hat{a} n_{1}^{2} n_{2}^{6} r_{1} \eta_{0}-96 \dot{\gamma} \hat{\beta} n_{1}^{2} n_{2}^{6} r_{1} \eta_{0}-$ $144 \dot{\gamma} n_{1}^{2} n_{2}^{2} r_{1}^{2} \eta_{0}-144 \dot{\gamma} \hat{a l} n_{1}^{2} n_{2}^{2} r_{1}^{2} \eta_{0}+216 \dot{\gamma} n_{1}^{4} n_{2}^{2} r_{1}^{2} \eta_{0}+288 \dot{\gamma}$ al $n_{1}^{4} n_{2}^{2} r_{1}^{2} \eta_{0}-48 \dot{\gamma} \hat{\beta} n_{1}^{4} n_{2}^{2} r_{1}^{2} \eta_{0}-$ $72 \dot{\gamma} n_{1}^{6} n_{2}^{2} r_{1}^{2} \eta_{0}-144 \dot{\gamma} a \hat{l} n_{1}^{6} n_{2}^{2} r_{1}^{2} \eta_{0}+48 \dot{\gamma} \hat{\beta} n_{1}^{6} n_{2}^{2} r_{1}^{2} \eta_{0}+216 \dot{\gamma} n_{1}^{2} n_{2}^{4} r_{1}^{2} \eta_{0}+288 \dot{\gamma}$ al $n_{1}^{2} n_{2}^{4} r_{1}^{2} \eta_{0}-$ $48 \dot{\gamma} \hat{\beta} n_{1}^{2} n_{2}^{4} r_{1}^{2} \eta_{0}-144 \dot{\gamma} n_{1}^{4} n_{2}^{4} r_{1}^{2} \eta_{0}-288 \dot{\gamma} \hat{a} \hat{l} n_{1}^{4} n_{2}^{4} r_{1}^{2} \eta_{0}+96 \dot{\gamma} \hat{\beta} n_{1}^{4} n_{2}^{4} n_{1}^{2} \eta_{0}-72 \dot{\gamma} n_{1}^{2} n_{2}^{6} n_{1}^{2} \eta_{0}-$ $144 \dot{\gamma} \hat{a} n_{1}^{2} n_{2}^{6} r_{1}^{2} \eta_{0}+48 \dot{\gamma} \hat{\beta} n_{1}^{2} n_{2}^{6} r_{1}^{2} \eta_{0}+18 \dot{\gamma} n_{1}^{2} r_{2} \eta_{0}+18 \dot{\gamma} \hat{a l} n_{1}^{2} r_{2} \eta_{0}-54 \dot{\gamma} n_{1}^{4} r_{2} \eta_{0}-54 \dot{\gamma} \hat{a} n_{1}^{4} r_{2} \eta_{0}+$ $18 \dot{\gamma} n_{2}^{2} r_{2} \eta_{0}+18 \dot{\gamma} a \hat{l} n_{2}^{2} r_{2} \eta_{0}-72 \dot{\gamma} n_{1}^{2} n_{2}^{2} r_{2} \eta_{0}-72 \dot{\gamma}$ all $n_{1}^{2} n_{2}^{2} r_{2} \eta_{0}+108 \dot{\gamma} n_{1}^{4} n_{2}^{2} r_{2} \eta_{0}+144 \dot{\gamma} \hat{a} n_{1}^{4} n_{2}^{2} r_{2} \eta_{0}-$ $36 \dot{\gamma} \hat{a} n_{1}^{6} n_{2}^{2} n_{2} \eta_{0}-54 \dot{\gamma} n_{2}^{4} n_{2} \eta_{0}-54 \dot{\gamma} a \hat{l} n_{2}^{4} r_{2} \eta_{0}+108 \dot{\gamma} n_{1}^{2} n_{2}^{4} n_{2} \eta_{0}+144 \dot{\gamma} a \hat{l} n_{1}^{2} n_{2}^{4} n_{2} \eta_{0}-$ $72 \dot{\gamma} a \hat{l} n_{1}^{4} n_{2}^{4} r_{2} \eta_{0}-36 \dot{\gamma} a \hat{1} n_{1}^{2} n_{2}^{6} r_{2} \eta_{0}+72 \dot{\gamma} n_{1}^{2} n_{2}^{2} r_{1} r_{2} \eta_{0}+72 \dot{\gamma}$ al $n_{1}^{2} n_{2}^{2} r_{1} r_{2} \eta_{0}-108 \dot{\gamma} n_{1}^{4} n_{2}^{2} r_{1} r_{2} \eta_{0}-$ $108 \dot{\gamma} a \hat{l} n_{1}^{4} n_{2}^{2} r_{1} r_{2} \eta_{0}+36 \dot{\gamma} a \hat{l} n_{1}^{6} n_{2}^{2} r_{1} r_{2} \eta_{0}-108 \dot{\gamma} n_{1}^{2} n_{2}^{4} r_{1} r_{2} \eta_{0}-108 \dot{\gamma} a l n_{1}^{2} n_{2}^{4} r_{1} r_{2} \eta_{0}+$ $72 \dot{\gamma} \hat{a} n_{1}^{4} n_{2}^{4} r_{1} r_{2} \eta_{0}+36 \dot{\gamma} \hat{a l} n_{1}^{2} n_{2}^{6} r_{1} r_{2} \eta_{0}-18 \dot{\gamma}^{2} n_{1}^{3} n_{2} \eta_{0} \theta_{0}+12 \dot{\gamma}^{2} \hat{\beta} n_{1}^{3} n_{2} \eta_{0} \theta_{0}-36 \dot{\gamma}^{2} n_{1}^{5} n_{2} \eta_{0} \theta_{0}-$ $54 \dot{\gamma}^{2} \hat{a} \hat{1} n_{1}^{5} n_{2} \eta_{0} \theta_{0}+24 \dot{\gamma}^{2} \hat{\beta} n_{1}^{5} n_{2} \eta_{0} \theta_{0}+18 \dot{\gamma}^{2} n_{1} n_{2}^{3} \eta_{0} \theta_{0}-12 \dot{\gamma}^{2} \hat{\beta} n_{1} n_{2}^{3} \eta_{0} \theta_{0}+36 \dot{\gamma}^{2} n_{1} n_{2}^{5} \eta_{0} \theta_{0}+$ $54 \dot{\gamma}^{2} \hat{a} 1 n_{1} n_{2}^{5} \eta_{0} \theta_{0}-24 \dot{\gamma}^{2} \hat{\beta} n_{1} n_{2}^{5} \eta_{0} \theta_{0}-36 \dot{\gamma}^{2} n_{1}^{3} n_{2} r_{1} \eta_{0} \theta_{0}-72 \dot{\gamma}^{2} \hat{a} 1 n_{1}^{3} n_{2} n_{1} \eta_{0} \theta_{0}+24 \dot{\gamma}^{2} \hat{\beta} n_{1}^{3} n_{2} r_{1} \eta_{0} \theta_{0}+$ $36 \dot{\gamma}^{2} n_{1}^{5} n_{2} r_{1} \eta_{0} \theta_{0}+72 \dot{\gamma}^{2} \hat{a} n_{1}^{5} n_{2} r_{1} \eta_{0} \theta_{0}-24 \dot{\gamma}^{2} \hat{\beta} n_{1}^{5} n_{2} r_{1} \eta_{0} \theta_{0}+36 \dot{\gamma}^{2} n_{1} n_{2}^{3} r_{1} \eta_{0} \theta_{0}+72 \dot{\gamma}^{2} \hat{a} 1 n_{1} n_{2}^{3} r_{1} \eta_{0} \theta_{0}-$ $24 \dot{\gamma}^{2} \hat{\beta} n_{1} n_{2}^{3} r_{1} \eta_{0} \theta_{0}-36 \dot{\gamma}^{2} n_{1} n_{2}^{5} r_{1} \eta_{0} \theta_{0}-72 \dot{\gamma}^{2} \hat{a} n_{1} n_{2}^{5} r_{1} \eta_{0} \theta_{0}+24 \dot{\gamma}^{2} \hat{\beta} n_{1} n_{2}^{5} r_{1} \eta_{0} \theta_{0}-$ $18 \dot{\gamma}^{2} \hat{a l} n_{1}^{5} n_{2} r_{2} \eta_{0} \theta_{0}+18 \dot{\gamma}^{2}$ all $\left.n_{1} n_{2}^{5} r_{2} \eta_{0} \theta_{0}\right)$ $\left(-12 r_{1}+18 n_{1}^{2} r_{1}-6 n_{1}^{4} r_{1}+12 n_{1}^{2} n_{2}^{2} r_{1}-24 n_{1}^{2} r_{1}^{2}+24 n_{1}^{4} r_{1}^{2}+24 n_{1}^{2} n_{2}^{2} r_{1}^{2}-24 n_{1}^{2} n_{2}^{2} r_{2}+6 n_{1}^{2} r_{1} r_{2}-\right.$ $\left.\left.18 n_{1}^{4} r_{1} r_{2}-12 n_{1}^{2} n_{2}^{2} r_{1} r_{2}+6 \dot{\gamma} n_{1} n_{2} \theta_{0}-6 \dot{\gamma} n_{1} n_{2} r_{2} \theta_{0}-6 \dot{\gamma}^{2} \theta_{0}^{2}\right)\right) /$

$\left(\left(12 n_{1} n_{2}-18 n_{1}^{3} n_{2}+6 n_{1}^{5} n_{2}-12 n_{1} n_{2}^{3}+6 n_{1}^{3} n_{2}^{3}-12 n_{1} n_{2} r_{1}+42 n_{1}^{3} n_{2} r_{1}-30 n_{1}^{5} n_{2} r_{1}+30 n_{1} n_{2}^{3} n_{1}-\right.\right.$ $30 n_{1}^{3} n_{2}^{3} n_{1}-24 n_{1}^{3} n_{2} r_{1}^{2}+24 n_{1}^{5} n_{2} r_{1}^{2}+24 n_{1}^{3} n_{2}^{3} r_{1}^{2}-6 n_{1}^{3} n_{2} r_{2}+18 n_{1}^{5} n_{2} r_{2}-12 n_{1} n_{2}^{3} r_{2}+18 n_{1}^{3} n_{2}^{3} r_{2}+$ $6 n_{1}^{3} n_{2} r_{1} r_{2}-18 n_{1}^{5} n_{2} r_{1} r_{2}-6 n_{1} n_{2}^{3} r_{1} r_{2}-18 n_{1}^{3} n_{2}^{3} r_{1} r_{2}+6 \dot{\gamma} \theta_{0}-9 \dot{\gamma} n_{1}^{2} \theta_{0}+3 \dot{\gamma} n_{1}^{4} \theta_{0}+3 \dot{\gamma} n_{2}^{2} \theta_{0}+$ $\left.3 \dot{\gamma} n_{1}^{2} n_{2}^{2} \theta_{0}+12 \dot{\gamma} n_{1}^{2} n_{1} \theta_{0}-12 \dot{\gamma} n_{1}^{4} n_{1} \theta_{0}-12 \dot{\gamma} n_{1}^{2} n_{2}^{2} n_{1} \theta_{0}-3 \dot{\gamma} n_{1}^{2} r_{2} \theta_{0}+9 \dot{\gamma} n_{1}^{4} n_{2} \theta_{0}-3 \dot{\gamma} n_{2}^{2} r_{2} \theta_{0}+9 \dot{\gamma} n_{1}^{2} n_{2}^{2} r_{2} \theta_{0}\right)$ $\left(-72 n_{1}^{2} n_{2}^{2}+144 n_{1}^{4} n_{2}^{2}-72 n_{1}^{6} n_{2}^{2}+144 n_{1}^{2} n_{2}^{4}-144 n_{1}^{4} n_{2}^{4}-72 n_{1}^{2} n_{2}^{6}+72 r_{1}-108 n_{1}^{2} r_{1}+36 n_{1}^{4} r_{1}-108 n_{2}^{2} r_{1}+\right.$ $288 n_{1}^{2} n_{2}^{2} r_{1}-648 n_{1}^{4} n_{2}^{2} n_{1}+432 n_{1}^{6} n_{2}^{2} r_{1}+36 n_{2}^{4} n_{1}-648 n_{1}^{2} n_{2}^{4} r_{1}+864 n_{1}^{4} n_{2}^{4} n_{1}+432 n_{1}^{2} n_{2}^{6} n_{1}+144 n_{1}^{2} r_{1}^{2}-$ $144 n_{1}^{4} r_{1}^{2}+144 n_{2}^{2} r_{1}^{2}-720 n_{1}^{2} n_{2}^{2} r_{1}^{2}+1080 n_{1}^{4} n_{2}^{2} r_{1}^{2}-648 n_{1}^{6} n_{2}^{2} r_{1}^{2}-144 n_{2}^{4} r_{1}^{2}+1080 n_{1}^{2} n_{2}^{4} r_{1}^{2}-1296 n_{1}^{4} n_{2}^{4} r_{1}^{2}-$ $648 n_{1}^{2} n_{2}^{6} r_{1}^{2}+288 n_{1}^{2} n_{2}^{2} r_{1}^{3}-576 n_{1}^{4} n_{2}^{2} r_{1}^{3}+288 n_{1}^{6} n_{2}^{2} r_{1}^{3}-576 n_{1}^{2} n_{2}^{4} r_{1}^{3}+576 n_{1}^{4} n_{2}^{4} r_{1}^{3}+288 n_{1}^{2} n_{2}^{6} r_{1}^{3}+$ $72 n_{1}^{2} n_{2}^{2} r_{2}+144 n_{1}^{4} n_{2}^{2} r_{2}-216 n_{1}^{6} n_{2}^{2} r_{2}+144 n_{1}^{2} n_{2}^{4} r_{2}-432 n_{1}^{4} n_{2}^{4} r_{2}-216 n_{1}^{2} n_{2}^{6} r_{2}-36 n_{1}^{2} r_{1} r_{2}+$ $108 n_{1}^{4} r_{1} r_{2}-36 n_{2}^{2} r_{1} r_{2}+288 n_{1}^{2} n_{2}^{2} r_{1} r_{2}-504 n_{1}^{4} n_{2}^{2} r_{1} r_{2}+432 n_{1}^{6} n_{2}^{2} r_{1} r_{2}+108 n_{2}^{4} r_{1} r_{2}-504 n_{1}^{2} n_{2}^{4} r_{1} r_{2}+$ $864 n_{1}^{4} n_{2}^{4} r_{1} r_{2}+432 n_{1}^{2} n_{2}^{6} r_{1} r_{2}-144 n_{1}^{2} n_{2}^{2} r_{1}^{2} r_{2}+360 n_{1}^{4} n_{2}^{2} r_{1}^{2} r_{2}-216 n_{1}^{6} n_{2}^{2} r_{1}^{2} r_{2}+360 n_{1}^{2} n_{2}^{4} r_{1}^{2} r_{2}-$ $432 n_{1}^{4} n_{2}^{4} r_{1}^{2} r_{2}-216 n_{1}^{2} n_{2}^{6} r_{1}^{2} r_{2}+72 \dot{\gamma}^{2} \theta_{0}^{2}-36 \dot{\gamma}^{2} n_{1}^{2} \theta_{0}^{2}+18 \dot{\gamma}^{2} n_{1}^{4} \theta_{0}^{2}-36 \dot{\gamma}^{2} n_{2}^{2} \theta_{0}^{2}+36 \dot{\gamma}^{2} n_{1}^{2} n_{2}^{2} \theta_{0}^{2}+$ $18 \dot{\gamma}^{2} n_{2}^{4} \theta_{0}^{2}+72 \dot{\gamma}^{2} n_{1}^{2} r_{1} \theta_{0}^{2}-72 \dot{\gamma}^{2} n_{1}^{4} r_{1} \theta_{0}^{2}+72 \dot{\gamma}^{2} n_{2}^{2} r_{1} \theta_{0}^{2}-144 \dot{\gamma}^{2} n_{1}^{2} n_{2}^{2} r_{1} \theta_{0}^{2}-72 \dot{\gamma}^{2} n_{2}^{4} r_{1} \theta_{0}^{2}-$ $\left.\left.36 \dot{\gamma}^{2} n_{1}^{2} r_{2} \theta_{0}^{2}+54 \dot{\gamma}^{2} n_{1}^{4} r_{2} \theta_{0}^{2}-36 \dot{\gamma}^{2} n_{2}^{2} r_{2} \theta_{0}^{2}+108 \dot{\gamma}^{2} n_{1}^{2} n_{2}^{2} r_{2} \theta_{0}^{2}+54 \dot{\gamma}^{2} n_{2}^{4} r_{2} \theta_{0}^{2}\right)\right)-$

$\left(\left(-2 n_{1} n_{2}+n_{1} n_{2}^{3}+2 n_{1} n_{2} r_{1}-4 n_{1} n_{2}^{3} r_{1}+3 n_{1} n_{2}^{3} r_{2}-\dot{\gamma} \theta_{0}\right)\right.$

$\left(\left(6 \dot{\gamma} \eta_{0}+6 \dot{\gamma} \hat{a} \eta_{0}-9 \dot{\gamma} n_{1}^{2} \eta_{0}-9 \dot{\gamma}\right.\right.$ âl $n_{1}^{2} \eta_{0}+3 \dot{\gamma} n_{1}^{4} \eta_{0}+3 \dot{\gamma} a \hat{l} n_{1}^{4} \eta_{0}-12 \dot{\gamma} n_{1}^{2} n_{2}^{2} \eta_{0}-12 \dot{\gamma}$ âl $n_{1}^{2} n_{2}^{2} \eta_{0}+$ $8 \dot{\gamma} \hat{\beta} n_{1}^{2} n_{2}^{2} \eta_{0}+6 \dot{\gamma} n_{1}^{4} n_{2}^{2} \eta_{0}+6 \dot{\gamma} \hat{a} \hat{l} n_{1}^{4} n_{2}^{2} \eta_{0}-4 \dot{\gamma} \hat{\beta} n_{1}^{4} n_{2}^{2} \eta_{0}+12 \dot{\gamma} n_{1}^{2} r_{1} \eta_{0}+12 \dot{\gamma} \hat{a} n_{1}^{2} r_{1} \eta_{0}-12 \dot{\gamma} n_{1}^{4} r_{1} \eta_{0}-$ $12 \dot{\gamma} \hat{a} n_{1}^{4} r_{1} \eta_{0}-6 \dot{\gamma} n_{1}^{2} n_{2}^{2} r_{1} \eta_{0}-12 \dot{\gamma} a \hat{l} n_{1}^{2} n_{2}^{2} r_{1} \eta_{0}+4 \dot{\gamma} \hat{\beta} n_{1}^{2} n_{2}^{2} r_{1} \eta_{0}-6 \dot{\gamma} n_{1}^{4} n_{2}^{2} r_{1} \eta_{0}+4 \dot{\gamma} \hat{\beta} n_{1}^{4} n_{2}^{2} r_{1} \eta_{0}-$ $3 \dot{\gamma} n_{1}^{2} r_{2} \eta_{0}-3 \dot{\gamma} a 1 n_{1}^{2} r_{2} \eta_{0}+9 \dot{\gamma} n_{1}^{4} r_{2} \eta_{0}+9 \dot{\gamma} a \hat{l} n_{1}^{4} r_{2} \eta_{0}-6 \dot{\gamma} a \hat{l} n_{1}^{4} n_{2}^{2} r_{2} \eta_{0}-3 \dot{\gamma}^{2} n_{1} n_{2} \eta_{0} \theta_{0}-$ $\left.6 \dot{\gamma}^{2} \hat{a} \hat{l} n_{1} n_{2} \eta_{0} \theta_{0}+2 \dot{\gamma}^{2} \hat{\beta} n_{1} n_{2} \eta_{0} \theta_{0}+9 \dot{\gamma}^{2} n_{1}^{3} n_{2} \eta_{0} \theta_{0}+12 \dot{\gamma}^{2} \hat{a} n_{1}^{3} n_{2} \eta_{0} \theta_{0}-6 \dot{\gamma}^{2} \hat{\beta} n_{1}^{3} n_{2} \eta_{0} \theta_{0}\right) /$ 
$\left(12 n_{1} n_{2}-18 n_{1}^{3} n_{2}+6 n_{1}^{5} n_{2}-12 n_{1} n_{2}^{3}+6 n_{1}^{3} n_{2}^{3}-12 n_{1} n_{2} r_{1}+42 n_{1}^{3} n_{2} r_{1}-30 n_{1}^{5} n_{2} r_{1}+30 n_{1} n_{2}^{3} r_{1}-30 n_{1}^{3} n_{2}^{3} r_{1}-\right.$ $24 n_{1}^{3} n_{2} r_{1}^{2}+24 n_{1}^{5} n_{2} r_{1}^{2}+24 n_{1}^{3} n_{2}^{3} r_{1}^{2}-6 n_{1}^{3} n_{2} r_{2}+18 n_{1}^{5} n_{2} r_{2}-12 n_{1} n_{2}^{3} r_{2}+18 n_{1}^{3} n_{2}^{3} r_{2}+6 n_{1}^{3} n_{2} r_{1} r_{2}-$ $18 n_{1}^{5} n_{2} r_{1} r_{2}-6 n_{1} n_{2}^{3} r_{1} r_{2}-18 n_{1}^{3} n_{2}^{3} r_{1} r_{2}+6 \dot{\gamma} \theta_{0}-9 \dot{\gamma} n_{1}^{2} \theta_{0}+3 \dot{\gamma} n_{1}^{4} \theta_{0}+3 \dot{\gamma} n_{2}^{2} \theta_{0}+3 \dot{\gamma} n_{1}^{2} n_{2}^{2} \theta_{0}+$ $\left.12 \dot{\gamma} n_{1}^{2} r_{1} \theta_{0}-12 \dot{\gamma} n_{1}^{4} r_{1} \theta_{0}-12 \dot{\gamma} n_{1}^{2} n_{2}^{2} r_{1} \theta_{0}-3 \dot{\gamma} n_{1}^{2} r_{2} \theta_{0}+9 \dot{\gamma} n_{1}^{4} r_{2} \theta_{0}-3 \dot{\gamma} n_{2}^{2} r_{2} \theta_{0}+9 \dot{\gamma} n_{1}^{2} n_{2}^{2} r_{2} \theta_{0}\right)-$ $\left(\left(-36 \dot{\gamma} \eta_{0}-36 \dot{\gamma}\right.\right.$ all $\eta_{0}+54 \dot{\gamma} n_{1}^{2} \eta_{0}+54 \dot{\gamma} \hat{a l} n_{1}^{2} \eta_{0}-18 \dot{\gamma} n_{1}^{4} \eta_{0}-18 \dot{\gamma} \hat{a l} n_{1}^{4} \eta_{0}+54 \dot{\gamma} n_{2}^{2} \eta_{0}+54 \dot{\gamma} \hat{a l} n_{2}^{2} \eta_{0}-$ $36 \dot{\gamma} n_{1}^{2} n_{2}^{2} \eta_{0}-72 \dot{\gamma} \hat{a} n_{1}^{2} n_{2}^{2} \eta_{0}-24 \dot{\gamma} \hat{\beta} n_{1}^{2} n_{2}^{2} \eta_{0}+72 \dot{\gamma} n_{1}^{4} n_{2}^{2} \eta_{0}+144 \dot{\gamma} a \hat{l} n_{1}^{4} n_{2}^{2} \eta_{0}-24 \dot{\gamma} \hat{\beta} n_{1}^{4} n_{2}^{2} \eta_{0}-$ $72 \dot{\gamma} n_{1}^{6} n_{2}^{2} \eta_{0}-108 \dot{\gamma} \hat{a l} n_{1}^{6} n_{2}^{2} \eta_{0}+48 \dot{\gamma} \hat{\beta} n_{1}^{6} n_{2}^{2} \eta_{0}-18 \dot{\gamma} n_{2}^{4} \eta_{0}-18 \dot{\gamma} \hat{a} 1 n_{2}^{4} \eta_{0}+72 \dot{\gamma} n_{1}^{2} n_{2}^{4} \eta_{0}+$ $144 \dot{\gamma} a 1 n_{1}^{2} n_{2}^{4} \eta_{0}-24 \dot{\gamma} \hat{\beta} n_{1}^{2} n_{2}^{4} \eta_{0}-144 \dot{\gamma} n_{1}^{4} n_{2}^{4} \eta_{0}-216 \dot{\gamma} \hat{a} n_{1}^{4} n_{2}^{4} \eta_{0}+96 \dot{\gamma} \hat{\beta} n_{1}^{4} n_{2}^{4} \eta_{0}-72 \dot{\gamma} n_{1}^{2} n_{2}^{6} \eta_{0}-$ $108 \dot{\gamma} \hat{a} n_{1}^{2} n_{2}^{6} \eta_{0}+48 \dot{\gamma} \hat{\beta} n_{1}^{2} n_{2}^{6} \eta_{0}-72 \dot{\gamma} n_{1}^{2} r_{1} \eta_{0}-72 \dot{\gamma}$ al $n_{1}^{2} r_{1} \eta_{0}+72 \dot{\gamma} n_{1}^{4} r_{1} \eta_{0}+72 \dot{\gamma} \hat{a} n_{1}^{4} r_{1} \eta_{0}-$ $72 \dot{\gamma} n_{2}^{2} r_{1} \eta_{0}-72 \dot{\gamma} \hat{a l} n_{2}^{2} r_{1} \eta_{0}+288 \dot{\gamma} n_{1}^{2} n_{2}^{2} r_{1} \eta_{0}+360 \dot{\gamma} \hat{a l} n_{1}^{2} n_{2}^{2} r_{1} \eta_{0}-48 \dot{\gamma} \hat{\beta} n_{1}^{2} n_{2}^{2} r_{1} \eta_{0}-$ $288 \dot{\gamma} n_{1}^{4} n_{2}^{2} r_{1} \eta_{0}-468 \dot{\gamma} \hat{a} n_{1}^{4} n_{2}^{2} r_{1} \eta_{0}+72 \dot{\gamma} \hat{\beta} n_{1}^{4} n_{2}^{2} r_{1} \eta_{0}+144 \dot{\gamma} n_{1}^{6} n_{2}^{2} r_{1} \eta_{0}+252 \dot{\gamma} \hat{l} n_{1}^{6} n_{2}^{2} r_{1} \eta_{0}-$ $96 \dot{\gamma} \hat{\beta} n_{1}^{6} n_{2}^{2} r_{1} \eta_{0}+72 \dot{\gamma} n_{2}^{4} r_{1} \eta_{0}+72 \dot{\gamma}$ al $n_{2}^{4} r_{1} \eta_{0}-288 \dot{\gamma} n_{1}^{2} n_{2}^{4} r_{1} \eta_{0}-468 \dot{\gamma}$ al $n_{1}^{2} n_{2}^{4} r_{1} \eta_{0}+$ $72 \dot{\gamma} \hat{\beta} n_{1}^{2} n_{2}^{4} r_{1} \eta_{0}+288 \dot{\gamma} n_{1}^{4} n_{2}^{4} r_{1} \eta_{0}+504 \dot{\gamma} \hat{a} 1 n_{1}^{4} n_{2}^{4} r_{1} \eta_{0}-192 \dot{\gamma} \hat{\beta} n_{1}^{4} n_{2}^{4} r_{1} \eta_{0}+144 \dot{\gamma} n_{1}^{2} n_{2}^{6} r_{1} \eta_{0}+$ $252 \dot{\gamma} \hat{a} 1 n_{1}^{2} n_{2}^{6} r_{1} \eta_{0}-96 \dot{\gamma} \hat{\beta} n_{1}^{2} n_{2}^{6} r_{1} \eta_{0}-144 \dot{\gamma} n_{1}^{2} n_{2}^{2} r_{1}^{2} \eta_{0}-144 \dot{\gamma} \hat{a} n_{1}^{2} n_{2}^{2} r_{1}^{2} \eta_{0}+216 \dot{\gamma} n_{1}^{4} n_{2}^{2} r_{1}^{2} \eta_{0}+$ $288 \dot{\gamma} \hat{a l} n_{1}^{4} n_{2}^{2} r_{1}^{2} \eta_{0}-48 \dot{\gamma} \hat{\beta} n_{1}^{4} n_{2}^{2} r_{1}^{2} \eta_{0}-72 \dot{\gamma} n_{1}^{6} n_{2}^{2} r_{1}^{2} \eta_{0}-144 \dot{\gamma} a \hat{l} n_{1}^{6} n_{2}^{2} r_{1}^{2} \eta_{0}+48 \dot{\gamma} \hat{\beta} n_{1}^{6} n_{2}^{2} r_{1}^{2} \eta_{0}+$ $216 \dot{\gamma} n_{1}^{2} n_{2}^{4} r_{1}^{2} \eta_{0}+288 \dot{\gamma} \hat{a} n_{1}^{2} n_{2}^{4} r_{1}^{2} \eta_{0}-48 \dot{\gamma} \hat{\beta} n_{1}^{2} n_{2}^{4} r_{1}^{2} \eta_{0}-144 \dot{\gamma} n_{1}^{4} n_{2}^{4} r_{1}^{2} \eta_{0}-288 \dot{\gamma} a \hat{l} n_{1}^{4} n_{2}^{4} r_{1}^{2} \eta_{0}+$ $96 \dot{\gamma} \hat{\beta} n_{1}^{4} n_{2}^{4} r_{1}^{2} \eta_{0}-72 \dot{\gamma} n_{1}^{2} n_{2}^{6} r_{1}^{2} \eta_{0}-144 \dot{\gamma} \hat{a} 1 n_{1}^{2} n_{2}^{6} r_{1}^{2} \eta_{0}+48 \dot{\gamma} \hat{\beta} n_{1}^{2} n_{2}^{6} r_{1}^{2} \eta_{0}+18 \dot{\gamma} n_{1}^{2} r_{2} \eta_{0}+$ $18 \dot{\gamma} \hat{a} n_{1}^{2} r_{2} \eta_{0}-54 \dot{\gamma} n_{1}^{4} r_{2} \eta_{0}-54 \dot{\gamma} \hat{a l} n_{1}^{4} r_{2} \eta_{0}+18 \dot{\gamma} n_{2}^{2} r_{2} \eta_{0}+18 \dot{\gamma}$ all $n_{2}^{2} r_{2} \eta_{0}-72 \dot{\gamma} n_{1}^{2} n_{2}^{2} r_{2} \eta_{0}-$ $72 \dot{\gamma}$ al $n_{1}^{2} n_{2}^{2} r_{2} \eta_{0}+108 \dot{\gamma} n_{1}^{4} n_{2}^{2} r_{2} \eta_{0}+144 \dot{\gamma} a 1 n_{1}^{4} n_{2}^{2} r_{2} \eta_{0}-36 \dot{\gamma}$ all $n_{1}^{6} n_{2}^{2} r_{2} \eta_{0}-54 \dot{\gamma} n_{2}^{4} r_{2} \eta_{0}-$ $54 \dot{\gamma} \hat{a} 1 n_{2}^{4} r_{2} \eta_{0}+108 \dot{\gamma} n_{1}^{2} n_{2}^{4} r_{2} \eta_{0}+144 \dot{\gamma}$ âl $n_{1}^{2} n_{2}^{4} r_{2} \eta_{0}-72 \dot{\gamma} \hat{a} 1 n_{1}^{4} n_{2}^{4} r_{2} \eta_{0}-36 \dot{\gamma}$ all $n_{1}^{2} n_{2}^{6} r_{2} \eta_{0}+$ $72 \dot{\gamma} n_{1}^{2} n_{2}^{2} r_{1} r_{2} \eta_{0}+72 \dot{\gamma} \hat{a} 1 n_{1}^{2} n_{2}^{2} r_{1} r_{2} \eta_{0}-108 \dot{\gamma} n_{1}^{4} n_{2}^{2} r_{1} r_{2} \eta_{0}-108 \dot{\gamma} \hat{a l} n_{1}^{4} n_{2}^{2} r_{1} r_{2} \eta_{0}+$ $36 \dot{\gamma} \hat{a} 1 n_{1}^{6} n_{2}^{2} r_{1} r_{2} \eta_{0}-108 \dot{\gamma} n_{1}^{2} n_{2}^{4} r_{1} r_{2} \eta_{0}-108 \dot{\gamma}$ al $n_{1}^{2} n_{2}^{4} r_{1} r_{2} \eta_{0}+72 \dot{\gamma} \hat{a l} n_{1}^{4} n_{2}^{4} r_{1} r_{2} \eta_{0}+$ $36 \dot{\gamma} \hat{a l} n_{1}^{2} n_{2}^{6} r_{1} r_{2} \eta_{0}-18 \dot{\gamma}^{2} n_{1}^{3} n_{2} \eta_{0} \theta_{0}+12 \dot{\gamma}^{2} \hat{\beta} n_{1}^{3} n_{2} \eta_{0} \theta_{0}-36 \dot{\gamma}^{2} n_{1}^{5} n_{2} \eta_{0} \theta_{0}-54 \dot{\gamma}^{2} \hat{a l} n_{1}^{5} n_{2} \eta_{0} \theta_{0}+$ $24 \dot{\gamma}^{2} \hat{\beta} n_{1}^{5} n_{2} \eta_{0} \theta_{0}+18 \dot{\gamma}^{2} n_{1} n_{2}^{3} \eta_{0} \theta_{0}-12 \dot{\gamma}^{2} \hat{\beta} n_{1} n_{2}^{3} \eta_{0} \theta_{0}+36 \dot{\gamma}^{2} n_{1} n_{2}^{5} \eta_{0} \theta_{0}+54 \dot{\gamma}^{2} \hat{a} 1 n_{1} n_{2}^{5} \eta_{0} \theta_{0}-$ $24 \dot{\gamma}^{2} \hat{\beta} n_{1} n_{2}^{5} \eta_{0} \theta_{0}-36 \dot{\gamma}^{2} n_{1}^{3} n_{2} r_{1} \eta_{0} \theta_{0}-72 \dot{\gamma}^{2} \hat{a} 1 n_{1}^{3} n_{2} r_{1} \eta_{0} \theta_{0}+24 \dot{\gamma}^{2} \hat{\beta} n_{1}^{3} n_{2} r_{1} \eta_{0} \theta_{0}+$ $36 \dot{\gamma}^{2} n_{1}^{5} n_{2} r_{1} \eta_{0} \theta_{0}+72 \dot{\gamma}^{2} \hat{a} 1 n_{1}^{5} n_{2} r_{1} \eta_{0} \theta_{0}-24 \dot{\gamma}^{2} \hat{\beta} n_{1}^{5} n_{2} r_{1} \eta_{0} \theta_{0}+36 \dot{\gamma}^{2} n_{1} n_{2}^{3} r_{1} \eta_{0} \theta_{0}+$ $72 \dot{\gamma}^{2} \hat{\mathrm{l}} \mathrm{n}_{1} \mathrm{n}_{2}^{3} \mathrm{r}_{1} \eta_{0} \theta_{0}-24 \dot{\gamma}^{2} \hat{\beta} n_{1} n_{2}^{3} r_{1} \eta_{0} \theta_{0}-36 \dot{\gamma}^{2} n_{1} n_{2}^{5} r_{1} \eta_{0} \theta_{0}-72 \dot{\gamma}^{2} \hat{a} n_{1} n_{2}^{5} r_{1} \eta_{0} \theta_{0}+$ $\left.24 \dot{\gamma}^{2} \hat{\beta} n_{1} n_{2}^{5} r_{1} \eta_{0} \theta_{0}-18 \dot{\gamma}^{2} \hat{a l} n_{1}^{5} n_{2} r_{2} \eta_{0} \theta_{0}+18 \dot{\gamma}^{2} \hat{a} n_{1} n_{2}^{5} r_{2} \eta_{0} \theta_{0}\right)$

$\left(-12 r_{1}+18 n_{1}^{2} r_{1}-6 n_{1}^{4} r_{1}+12 n_{1}^{2} n_{2}^{2} r_{1}-24 n_{1}^{2} r_{1}^{2}+24 n_{1}^{4} r_{1}^{2}+24 n_{1}^{2} n_{2}^{2} r_{1}^{2}-24 n_{1}^{2} n_{2}^{2} r_{2}+6 n_{1}^{2} r_{1} r_{2}-\right.$ $\left.\left.18 n_{1}^{4} r_{1} r_{2}-12 n_{1}^{2} n_{2}^{2} r_{1} r_{2}+6 \dot{\gamma} n_{1} n_{2} \theta_{0}-6 \dot{\gamma} n_{1} n_{2} r_{2} \theta_{0}-6 \dot{\gamma}^{2} \theta_{0}^{2}\right)\right) /$

( $\left(12 n_{1} n_{2}-18 n_{1}^{3} n_{2}+6 n_{1}^{5} n_{2}-12 n_{1} n_{2}^{3}+6 n_{1}^{3} n_{2}^{3}-12 n_{1} n_{2} r_{1}+42 n_{1}^{3} n_{2} r_{1}-30 n_{1}^{5} n_{2} r_{1}+30 n_{1} n_{2}^{3} r_{1}-\right.$ $30 n_{1}^{3} n_{2}^{3} r_{1}-24 n_{1}^{3} n_{2} r_{1}^{2}+24 n_{1}^{5} n_{2} r_{1}^{2}+24 n_{1}^{3} n_{2}^{3} r_{1}^{2}-6 n_{1}^{3} n_{2} r_{2}+18 n_{1}^{5} n_{2} r_{2}-12 n_{1} n_{2}^{3} r_{2}+18 n_{1}^{3} n_{2}^{3} r_{2}+$ $6 n_{1}^{3} n_{2} r_{1} r_{2}-18 n_{1}^{5} n_{2} r_{1} r_{2}-6 n_{1} n_{2}^{3} r_{1} r_{2}-18 n_{1}^{3} n_{2}^{3} r_{1} r_{2}+6 \dot{\gamma} \theta_{0}-9 \dot{\gamma} n_{1}^{2} \theta_{0}+3 \dot{\gamma} n_{1}^{4} \theta_{0}+3 \dot{\gamma} n_{2}^{2} \theta_{0}+$ $3 \dot{\gamma} n_{1}^{2} n_{2}^{2} \theta_{0}+12 \dot{\gamma} n_{1}^{2} r_{1} \theta_{0}-12 \dot{\gamma} n_{1}^{4} r_{1} \theta_{0}-12 \dot{\gamma} n_{1}^{2} n_{2}^{2} r_{1} \theta_{0}-3 \dot{\gamma} n_{1}^{2} r_{2} \theta_{0}+9 \dot{\gamma} n_{1}^{4} r_{2} \theta_{0}-3 \dot{\gamma} n_{2}^{2} r_{2} \theta_{0}+$ $\left.9 \dot{\gamma} n_{1}^{2} n_{2}^{2} r_{2} \theta_{0}\right)\left(-72 n_{1}^{2} n_{2}^{2}+144 n_{1}^{4} n_{2}^{2}-72 n_{1}^{6} n_{2}^{2}+144 n_{1}^{2} n_{2}^{4}-144 n_{1}^{4} n_{2}^{4}-72 n_{1}^{2} n_{2}^{6}+72 r_{1}-108 n_{1}^{2} r_{1}+\right.$ $36 n_{1}^{4} r_{1}-108 n_{2}^{2} r_{1}+288 n_{1}^{2} n_{2}^{2} r_{1}-648 n_{1}^{4} n_{2}^{2} r_{1}+432 n_{1}^{6} n_{2}^{2} r_{1}+36 n_{2}^{4} r_{1}-648 n_{1}^{2} n_{2}^{4} r_{1}+864 n_{1}^{4} n_{2}^{4} r_{1}+$ $432 n_{1}^{2} n_{2}^{6} r_{1}+144 n_{1}^{2} r_{1}^{2}-144 n_{1}^{4} r_{1}^{2}+144 n_{2}^{2} r_{1}^{2}-720 n_{1}^{2} n_{2}^{2} r_{1}^{2}+1080 n_{1}^{4} n_{2}^{2} r_{1}^{2}-648 n_{1}^{6} n_{2}^{2} r_{1}^{2}-144 n_{2}^{4} r_{1}^{2}+$ $1080 n_{1}^{2} n_{2}^{4} r_{1}^{2}-1296 n_{1}^{4} n_{2}^{4} r_{1}^{2}-648 n_{1}^{2} n_{2}^{6} r_{1}^{2}+288 n_{1}^{2} n_{2}^{2} r_{1}^{3}-576 n_{1}^{4} n_{2}^{2} r_{1}^{3}+288 n_{1}^{6} n_{2}^{2} r_{1}^{3}-576 n_{1}^{2} n_{2}^{4} r_{1}^{3}+$ $576 n_{1}^{4} n_{2}^{4} r_{1}^{3}+288 n_{1}^{2} n_{2}^{6} r_{1}^{3}+72 n_{1}^{2} n_{2}^{2} r_{2}+144 n_{1}^{4} n_{2}^{2} r_{2}-216 n_{1}^{6} n_{2}^{2} r_{2}+144 n_{1}^{2} n_{2}^{4} r_{2}-432 n_{1}^{4} n_{2}^{4} r_{2}-$ $216 n_{1}^{2} n_{2}^{6} r_{2}-36 n_{1}^{2} r_{1} r_{2}+108 n_{1}^{4} r_{1} r_{2}-36 n_{2}^{2} r_{1} r_{2}+288 n_{1}^{2} n_{2}^{2} r_{1} r_{2}-504 n_{1}^{4} n_{2}^{2} r_{1} r_{2}+432 n_{1}^{6} n_{2}^{2} r_{1} r_{2}+$ $108 n_{2}^{4} r_{1} r_{2}-504 n_{1}^{2} n_{2}^{4} r_{1} r_{2}+864 n_{1}^{4} n_{2}^{4} r_{1} r_{2}+432 n_{1}^{2} n_{2}^{6} r_{1} r_{2}-144 n_{1}^{2} n_{2}^{2} r_{1}^{2} r_{2}+360 n_{1}^{4} n_{2}^{2} r_{1}^{2} r_{2}-$ $216 n_{1}^{6} n_{2}^{2} r_{1}^{2} r_{2}+360 n_{1}^{2} n_{2}^{4} r_{1}^{2} r_{2}-432 n_{1}^{4} n_{2}^{4} r_{1}^{2} r_{2}-216 n_{1}^{2} n_{2}^{6} r_{1}^{2} r_{2}+72 \dot{\gamma}^{2} \theta_{0}^{2}-36 \dot{\gamma}^{2} n_{1}^{2} \theta_{0}^{2}+18 \dot{\gamma}^{2} n_{1}^{4} \theta_{0}^{2}-$ $36 \dot{\gamma}^{2} n_{2}^{2} \theta_{0}^{2}+36 \dot{\gamma}^{2} n_{1}^{2} n_{2}^{2} \theta_{0}^{2}+18 \dot{\gamma}^{2} n_{2}^{4} \theta_{0}^{2}+72 \dot{\gamma}^{2} n_{1}^{2} r_{1} \theta_{0}^{2}-72 \dot{\gamma}^{2} n_{1}^{4} r_{1} \theta_{0}^{2}+72 \dot{\gamma}^{2} n_{2}^{2} r_{1} \theta_{0}^{2}-$

$144 \dot{\gamma}^{2} n_{1}^{2} n_{2}^{2} r_{1} \theta_{0}^{2}-72 \dot{\gamma}^{2} n_{2}^{4} r_{1} \theta_{0}^{2}-36 \dot{\gamma}^{2} n_{1}^{2} r_{2} \theta_{0}^{2}+54 \dot{\gamma}^{2} n_{1}^{4} r_{2} \theta_{0}^{2}-36 \dot{\gamma}^{2} n_{2}^{2} r_{2} \theta_{0}^{2}+108 \dot{\gamma}^{2} n_{1}^{2} n_{2}^{2} r_{2} \theta_{0}^{2}+$ $\left.\left.\left.54 \dot{\gamma}^{2} n_{2}^{4} r_{2} \theta_{0}^{2}\right)\right)\right)\left(/\left(-2 n_{1} n_{2}+n_{1}^{3} n_{2}+2 n_{1} n_{2} r_{1}-4 n_{1}^{3} n_{2} r_{1}+3 n_{1}^{3} n_{2} r_{2}+\dot{\gamma} \theta_{0}\right)\right.$ ， 
$\sigma_{11} \rightarrow-\frac{-\dot{\gamma} \eta_{0}-\dot{\gamma} \hat{a} l \eta_{0}+3 \dot{\gamma} n_{1}^{2} n_{2}^{2} \eta_{0}+4 \dot{\gamma} a \hat{l} n_{1}^{2} n_{2}^{2} \eta_{0}-2 \dot{\gamma} \hat{\beta} n_{1}^{2} n_{2}^{2} \eta_{0}}{-2 n_{1} n_{2}+n_{1}^{3} n_{2}+2 n_{1} n_{2} n_{1}-4 n_{1}^{3} n_{2} n_{1}+3 n_{1}^{3} n_{2} r_{2}+\dot{\gamma} \theta_{0}}+$
$\left(\left(2 n_{1}^{2} n_{2}^{2}+2 n_{1}-8 n_{1}^{2} n_{2}^{2} n_{1}+6 n_{1}^{2} n_{2}^{2} n_{2}\right)\right.$

$\left(-36 \dot{\gamma} \eta_{0}-36 \dot{\gamma} \hat{a l l} \eta_{0}+54 \dot{\gamma} n_{1}^{2} \eta_{0}+54 \dot{\gamma}\right.$ all $n_{1}^{2} \eta_{0}-18 \dot{\gamma} n_{1}^{4} \eta_{0}-18 \dot{\gamma}$ all $n_{1}^{4} \eta_{0}+54 \dot{\gamma} n_{2}^{2} \eta_{0}+54 \dot{\gamma} \hat{a} n_{2}^{2} \eta_{0}-$

$36 \dot{\gamma} n_{1}^{2} n_{2}^{2} \eta_{0}-72 \dot{\gamma} \hat{a} n_{1}^{2} n_{2}^{2} \eta_{0}-24 \dot{\gamma} \hat{\beta} n_{1}^{2} n_{2}^{2} \eta_{0}+72 \dot{\gamma} n_{1}^{4} n_{2}^{2} \eta_{0}+144 \dot{\gamma} \hat{a} n_{1}^{4} n_{2}^{2} \eta_{0}-24 \dot{\gamma} \hat{\beta} n_{1}^{4} n_{2}^{2} \eta_{0}-$

$72 \dot{\gamma} n_{1}^{6} n_{2}^{2} \eta_{0}-108 \dot{\gamma} \hat{a} 1 n_{1}^{6} n_{2}^{2} \eta_{0}+48 \dot{\gamma} \hat{\beta} n_{1}^{6} n_{2}^{2} \eta_{0}-18 \dot{\gamma} n_{2}^{4} \eta_{0}-18 \dot{\gamma} \hat{a} n_{2}^{4} \eta_{0}+72 \dot{\gamma} n_{1}^{2} n_{2}^{4} \eta_{0}+$

$144 \dot{\gamma} \hat{a} n_{1}^{2} n_{2}^{4} \eta_{0}-24 \dot{\gamma} \hat{\beta} n_{1}^{2} n_{2}^{4} \eta_{0}-144 \dot{\gamma} n_{1}^{4} n_{2}^{4} \eta_{0}-216 \dot{\gamma} \hat{a l} n_{1}^{4} n_{2}^{4} \eta_{0}+96 \dot{\gamma} \hat{\beta} n_{1}^{4} n_{2}^{4} \eta_{0}-72 \dot{\gamma} n_{1}^{2} n_{2}^{6} \eta_{0}-$

$108 \dot{\gamma} \hat{a} n_{1}^{2} n_{2}^{6} \eta_{0}+48 \dot{\gamma} \hat{\beta} n_{1}^{2} n_{2}^{6} \eta_{0}-72 \dot{\gamma} n_{1}^{2} r_{1} \eta_{0}-72 \dot{\gamma} \hat{a} n_{1}^{2} r_{1} \eta_{0}+72 \dot{\gamma} n_{1}^{4} r_{1} \eta_{0}+72 \dot{\gamma} \hat{a} n_{1}^{4} r_{1} \eta_{0}-$

$72 \dot{\gamma} n_{2}^{2} r_{1} \eta_{0}-72 \dot{\gamma} a \hat{l} n_{2}^{2} r_{1} \eta_{0}+288 \dot{\gamma} n_{1}^{2} n_{2}^{2} r_{1} \eta_{0}+360 \dot{\gamma}$ al $n_{1}^{2} n_{2}^{2} r_{1} \eta_{0}-48 \dot{\gamma} \hat{\beta} n_{1}^{2} n_{2}^{2} r_{1} \eta_{0}-$

$288 \dot{\gamma} n_{1}^{4} n_{2}^{2} r_{1} \eta_{0}-468 \dot{\gamma}$ âl $n_{1}^{4} n_{2}^{2} r_{1} \eta_{0}+72 \dot{\gamma} \hat{\beta} n_{1}^{4} n_{2}^{2} r_{1} \eta_{0}+144 \dot{\gamma} n_{1}^{6} n_{2}^{2} r_{1} \eta_{0}+252 \dot{\gamma}$ âl $n_{1}^{6} n_{2}^{2} r_{1} \eta_{0}-$

$96 \dot{\gamma} \hat{\beta} n_{1}^{6} n_{2}^{2} r_{1} \eta_{0}+72 \dot{\gamma} n_{2}^{4} r_{1} \eta_{0}+72 \dot{\gamma} a \hat{l} n_{2}^{4} r_{1} \eta_{0}-288 \dot{\gamma} n_{1}^{2} n_{2}^{4} r_{1} \eta_{0}-468 \dot{\gamma} a \hat{a} n_{1}^{2} n_{2}^{4} r_{1} \eta_{0}+$

$72 \dot{\gamma} \hat{\beta} n_{1}^{2} n_{2}^{4} r_{1} \eta_{0}+288 \dot{\gamma} n_{1}^{4} n_{2}^{4} r_{1} \eta_{0}+504 \dot{\gamma} \hat{a} n_{1}^{4} n_{2}^{4} r_{1} \eta_{0}-192 \dot{\gamma} \hat{\beta} n_{1}^{4} n_{2}^{4} r_{1} \eta_{0}+144 \dot{\gamma} n_{1}^{2} n_{2}^{6} r_{1} \eta_{0}+$

$252 \dot{\gamma} a 1 n_{1}^{2} n_{2}^{6} r_{1} \eta_{0}-96 \dot{\gamma} \hat{\beta} n_{1}^{2} n_{2}^{6} n_{1} \eta_{0}-144 \dot{\gamma} n_{1}^{2} n_{2}^{2} n_{1}^{2} \eta_{0}-144 \dot{\gamma}$ al $n_{1}^{2} n_{2}^{2} n_{1}^{2} \eta_{0}+216 \dot{\gamma} n_{1}^{4} n_{2}^{2} r_{1}^{2} \eta_{0}+$

$288 \dot{\gamma} \hat{a} n_{1}^{4} n_{2}^{2} r_{1}^{2} \eta_{0}-48 \dot{\gamma} \hat{\beta} n_{1}^{4} n_{2}^{2} n_{1}^{2} \eta_{0}-72 \dot{\gamma} n_{1}^{6} n_{2}^{2} r_{1}^{2} \eta_{0}-144 \dot{\gamma} \hat{a} n_{1}^{6} n_{2}^{2} n_{1}^{2} \eta_{0}+48 \dot{\gamma} \hat{\beta} n_{1}^{6} n_{2}^{2} n_{1}^{2} \eta_{0}+$

$216 \dot{\gamma} n_{1}^{2} n_{2}^{4} r_{1}^{2} \eta_{0}+288 \dot{\gamma} \hat{a} n_{1}^{2} n_{2}^{4} r_{1}^{2} \eta_{0}-48 \dot{\gamma} \hat{\beta} n_{1}^{2} n_{2}^{4} r_{1}^{2} \eta_{0}-144 \dot{\gamma} n_{1}^{4} n_{2}^{4} r_{1}^{2} \eta_{0}-288 \dot{\gamma} \hat{l} n_{1}^{4} n_{2}^{4} r_{1}^{2} \eta_{0}+$

$96 \dot{\gamma} \hat{\beta} n_{1}^{4} n_{2}^{4} r_{1}^{2} \eta_{0}-72 \dot{\gamma} n_{1}^{2} n_{2}^{6} n_{1}^{2} \eta_{0}-144 \dot{\gamma} \hat{a} 1 n_{1}^{2} n_{2}^{6} r_{1}^{2} \eta_{0}+48 \dot{\gamma} \hat{\beta} n_{1}^{2} n_{2}^{6} r_{1}^{2} \eta_{0}+18 \dot{\gamma} n_{1}^{2} n_{2} \eta_{0}+$

$18 \dot{\gamma} \hat{a} n_{1}^{2} r_{2} \eta_{0}-54 \dot{\gamma} n_{1}^{4} r_{2} \eta_{0}-54 \dot{\gamma}$ al $n_{1}^{4} r_{2} \eta_{0}+18 \dot{\gamma} n_{2}^{2} r_{2} \eta_{0}+18 \dot{\gamma}$ âl $n_{2}^{2} r_{2} \eta_{0}-72 \dot{\gamma} n_{1}^{2} n_{2}^{2} r_{2} \eta_{0}-$

$72 \dot{\gamma} a \hat{l} n_{1}^{2} n_{2}^{2} r_{2} \eta_{0}+108 \dot{\gamma} n_{1}^{4} n_{2}^{2} n_{2} \eta_{0}+144 \dot{\gamma} a \hat{a} n_{1}^{4} n_{2}^{2} n_{2} \eta_{0}-36 \dot{\gamma} a \hat{l} n_{1}^{6} n_{2}^{2} n_{2} \eta_{0}-54 \dot{\gamma} n_{2}^{4} n_{2} \eta_{0}-$

$54 \dot{\gamma} \hat{a} n_{2}^{4} r_{2} \eta_{0}+108 \dot{\gamma} n_{1}^{2} n_{2}^{4} r_{2} \eta_{0}+144 \dot{\gamma}$ âl $n_{1}^{2} n_{2}^{4} r_{2} \eta_{0}-72 \dot{\gamma} \hat{a} n_{1}^{4} n_{2}^{4} r_{2} \eta_{0}-36 \dot{\gamma} a \hat{l} n_{1}^{2} n_{2}^{6} r_{2} \eta_{0}+$

$72 \dot{\gamma} n_{1}^{2} n_{2}^{2} r_{1} r_{2} \eta_{0}+72 \dot{\gamma} \hat{a} n_{1}^{2} n_{2}^{2} r_{1} r_{2} \eta_{0}-108 \dot{\gamma} n_{1}^{4} n_{2}^{2} r_{1} r_{2} \eta_{0}-108 \dot{\gamma} \hat{a} n_{1}^{4} n_{2}^{2} r_{1} r_{2} \eta_{0}+$

$36 \dot{\gamma}$ al $n_{1}^{6} n_{2}^{2} r_{1} r_{2} \eta_{0}-108 \dot{\gamma} n_{1}^{2} n_{2}^{4} r_{1} r_{2} \eta_{0}-108 \dot{\gamma}$ al $n_{1}^{2} n_{2}^{4} r_{1} r_{2} \eta_{0}+72 \dot{\gamma}$ âl $n_{1}^{4} n_{2}^{4} r_{1} r_{2} \eta_{0}+$

$36 \dot{\gamma} \hat{a} n_{1}^{2} n_{2}^{6} r_{1} r_{2} \eta_{0}-18 \dot{\gamma}^{2} n_{1}^{3} n_{2} \eta_{0} \theta_{0}+12 \dot{\gamma}^{2} \hat{\beta} n_{1}^{3} n_{2} \eta_{0} \theta_{0}-36 \dot{\gamma}^{2} n_{1}^{5} n_{2} \eta_{0} \theta_{0}-54 \dot{\gamma}^{2}$ al $n_{1}^{5} n_{2} \eta_{0} \theta_{0}+$

$24 \dot{\gamma}^{2} \hat{\beta} n_{1}^{5} n_{2} \eta_{0} \theta_{0}+18 \dot{\gamma}^{2} n_{1} n_{2}^{3} \eta_{0} \theta_{0}-12 \dot{\gamma}^{2} \hat{\beta} n_{1} n_{2}^{3} \eta_{0} \theta_{0}+36 \dot{\gamma}^{2} n_{1} n_{2}^{5} \eta_{0} \theta_{0}+54 \dot{\gamma}^{2} \hat{a} 1 n_{1} n_{2}^{5} \eta_{0} \theta_{0}-$

$24 \dot{\gamma}^{2} \hat{\beta} n_{1} n_{2}^{5} \eta_{0} \theta_{0}-36 \dot{\gamma}^{2} n_{1}^{3} n_{2} r_{1} \eta_{0} \theta_{0}-72 \dot{\gamma}^{2} \hat{a} 1 n_{1}^{3} n_{2} r_{1} \eta_{0} \theta_{0}+24 \dot{\gamma}^{2} \hat{\beta} n_{1}^{3} n_{2} r_{1} \eta_{0} \theta_{0}+36 \dot{\gamma}^{2} n_{1}^{5} n_{2} r_{1} \eta_{0} \theta_{0}+$

$72 \dot{\gamma}^{2} \hat{a} 1 n_{1}^{5} n_{2} r_{1} \eta_{0} \theta_{0}-24 \dot{\gamma}^{2} \hat{\beta} n_{1}^{5} n_{2} r_{1} \eta_{0} \theta_{0}+36 \dot{\gamma}^{2} n_{1} n_{2}^{3} r_{1} \eta_{0} \theta_{0}+72 \dot{\gamma}^{2} \hat{a} n_{1} n_{2}^{3} r_{1} \eta_{0} \theta_{0}-$

$24 \dot{\gamma}^{2} \hat{\beta} n_{1} n_{2}^{3} n_{1} \eta_{0} \theta_{0}-36 \dot{\gamma}^{2} n_{1} n_{2}^{5} n_{1} \eta_{0} \theta_{0}-72 \dot{\gamma}^{2} \hat{a} n_{1} n_{2}^{5} n_{1} \eta_{0} \theta_{0}+24 \dot{\gamma}^{2} \hat{\beta} n_{1} n_{2}^{5} n_{1} \eta_{0} \theta_{0}-$

$\left.\left.18 \dot{\gamma}^{2} a \hat{l} n_{1}^{5} n_{2} r_{2} \eta_{0} \theta_{0}+18 \dot{\gamma}^{2} \hat{a l} n_{1} n_{2}^{5} n_{2} \eta_{0} \theta_{0}\right)\right) /$

$\left(\left(-2 n_{1} n_{2}+n_{1}^{3} n_{2}+2 n_{1} n_{2} r_{1}-4 n_{1}^{3} n_{2} r_{1}+3 n_{1}^{3} n_{2} r_{2}+\dot{\gamma} \theta_{0}\right)\right.$

$\left(-72 n_{1}^{2} n_{2}^{2}+144 n_{1}^{4} n_{2}^{2}-72 n_{1}^{6} n_{2}^{2}+144 n_{1}^{2} n_{2}^{4}-144 n_{1}^{4} n_{2}^{4}-72 n_{1}^{2} n_{2}^{6}+72 r_{1}-108 n_{1}^{2} r_{1}+36 n_{1}^{4} r_{1}-108 n_{2}^{2} r_{1}+\right.$

$288 n_{1}^{2} n_{2}^{2} r_{1}-648 n_{1}^{4} n_{2}^{2} r_{1}+432 n_{1}^{6} n_{2}^{2} r_{1}+36 n_{2}^{4} r_{1}-648 n_{1}^{2} n_{2}^{4} r_{1}+864 n_{1}^{4} n_{2}^{4} r_{1}+432 n_{1}^{2} n_{2}^{6} r_{1}+144 n_{1}^{2} r_{1}^{2}-$

$144 n_{1}^{4} r_{1}^{2}+144 n_{2}^{2} r_{1}^{2}-720 n_{1}^{2} n_{2}^{2} r_{1}^{2}+1080 n_{1}^{4} n_{2}^{2} r_{1}^{2}-648 n_{1}^{6} n_{2}^{2} r_{1}^{2}-144 n_{2}^{4} r_{1}^{2}+1080 n_{1}^{2} n_{2}^{4} r_{1}^{2}-1296 n_{1}^{4} n_{2}^{4} r_{1}^{2}-$

$648 n_{1}^{2} n_{2}^{6} r_{1}^{2}+288 n_{1}^{2} n_{2}^{2} r_{1}^{3}-576 n_{1}^{4} n_{2}^{2} r_{1}^{3}+288 n_{1}^{6} n_{2}^{2} r_{1}^{3}-576 n_{1}^{2} n_{2}^{4} r_{1}^{3}+576 n_{1}^{4} n_{2}^{4} r_{1}^{3}+288 n_{1}^{2} n_{2}^{6} r_{1}^{3}+$

$72 n_{1}^{2} n_{2}^{2} r_{2}+144 n_{1}^{4} n_{2}^{2} r_{2}-216 n_{1}^{6} n_{2}^{2} r_{2}+144 n_{1}^{2} n_{2}^{4} r_{2}-432 n_{1}^{4} n_{2}^{4} r_{2}-216 n_{1}^{2} n_{2}^{6} r_{2}-36 n_{1}^{2} r_{1} r_{2}+$

$108 n_{1}^{4} r_{1} r_{2}-36 n_{2}^{2} r_{1} r_{2}+288 n_{1}^{2} n_{2}^{2} r_{1} r_{2}-504 n_{1}^{4} n_{2}^{2} r_{1} r_{2}+432 n_{1}^{6} n_{2}^{2} r_{1} r_{2}+108 n_{2}^{4} r_{1} r_{2}-504 n_{1}^{2} n_{2}^{4} r_{1} r_{2}+$

$864 n_{1}^{4} n_{2}^{4} r_{1} r_{2}+432 n_{1}^{2} n_{2}^{6} r_{1} r_{2}-144 n_{1}^{2} n_{2}^{2} r_{1}^{2} r_{2}+360 n_{1}^{4} n_{2}^{2} r_{1}^{2} r_{2}-216 n_{1}^{6} n_{2}^{2} r_{1}^{2} r_{2}+360 n_{1}^{2} n_{2}^{4} r_{1}^{2} r_{2}-$

$432 n_{1}^{4} n_{2}^{4} r_{1}^{2} r_{2}-216 n_{1}^{2} n_{2}^{6} r_{1}^{2} r_{2}+72 \dot{\gamma}^{2} \theta_{0}^{2}-36 \dot{\gamma}^{2} n_{1}^{2} \theta_{0}^{2}+18 \dot{\gamma}^{2} n_{1}^{4} \theta_{0}^{2}-36 \dot{\gamma}^{2} n_{2}^{2} \theta_{0}^{2}+36 \dot{\gamma}^{2} n_{1}^{2} n_{2}^{2} \theta_{0}^{2}+$

$18 \dot{\gamma}^{2} n_{2}^{4} \theta_{0}^{2}+72 \dot{\gamma}^{2} n_{1}^{2} r_{1} \theta_{0}^{2}-72 \dot{\gamma}^{2} n_{1}^{4} r_{1} \theta_{0}^{2}+72 \dot{\gamma}^{2} n_{2}^{2} r_{1} \theta_{0}^{2}-144 \dot{\gamma}^{2} n_{1}^{2} n_{2}^{2} r_{1} \theta_{0}^{2}-72 \dot{\gamma}^{2} n_{2}^{4} r_{1} \theta_{0}^{2}-$

$\left.\left.36 \dot{\gamma}^{2} n_{1}^{2} r_{2} \theta_{0}^{2}+54 \dot{\gamma}^{2} n_{1}^{4} r_{2} \theta_{0}^{2}-36 \dot{\gamma}^{2} n_{2}^{2} r_{2} \theta_{0}^{2}+108 \dot{\gamma}^{2} n_{1}^{2} n_{2}^{2} r_{2} \theta_{0}^{2}+54 \dot{\gamma}^{2} n_{2}^{4} r_{2} \theta_{0}^{2}\right)\right)+$

$\left(\left(-2 n_{1} n_{2}+n_{1} n_{2}^{3}+2 n_{1} n_{2} n_{1}-4 n_{1} n_{2}^{3} n_{1}+3 n_{1} n_{2}^{3} n_{2}-\dot{\gamma} \theta_{0}\right)\right.$

$\left(\left(6 \dot{\gamma} \eta_{0}+6 \dot{\gamma} \hat{a l} \eta_{0}-9 \dot{\gamma} n_{1}^{2} \eta_{0}-9 \dot{\gamma} \hat{a l} n_{1}^{2} \eta_{0}+3 \dot{\gamma} n_{1}^{4} \eta_{0}+3 \dot{\gamma} \hat{a} n_{1}^{4} \eta_{0}-12 \dot{\gamma} n_{1}^{2} n_{2}^{2} \eta_{0}-12 \dot{\gamma} a \hat{l} n_{1}^{2} n_{2}^{2} \eta_{0}+\right.\right.$

$8 \dot{\gamma} \hat{\beta} n_{1}^{2} n_{2}^{2} \eta_{0}+6 \dot{\gamma} n_{1}^{4} n_{2}^{2} \eta_{0}+6 \dot{\gamma} \hat{a} 1 n_{1}^{4} n_{2}^{2} \eta_{0}-4 \dot{\gamma} \hat{\beta} n_{1}^{4} n_{2}^{2} \eta_{0}+12 \dot{\gamma} n_{1}^{2} n_{1} \eta_{0}+12 \dot{\gamma} a \hat{l} n_{1}^{2} n_{1} \eta_{0}-$

$12 \dot{\gamma} n_{1}^{4} r_{1} \eta_{0}-12 \dot{\gamma} a \hat{a} n_{1}^{4} r_{1} \eta_{0}-6 \dot{\gamma} n_{1}^{2} n_{2}^{2} r_{1} \eta_{0}-12 \dot{\gamma} a \hat{l} n_{1}^{2} n_{2}^{2} r_{1} \eta_{0}+4 \dot{\gamma} \hat{\beta} n_{1}^{2} n_{2}^{2} r_{1} \eta_{0}-6 \dot{\gamma} n_{1}^{4} n_{2}^{2} r_{1} \eta_{0}+$

$4 \dot{\gamma} \hat{\beta} n_{1}^{4} n_{2}^{2} n_{1} \eta_{0}-3 \dot{\gamma} n_{1}^{2} n_{2} \eta_{0}-3 \dot{\gamma} a \hat{a} n_{1}^{2} n_{2} \eta_{0}+9 \dot{\gamma} n_{1}^{4} n_{2} \eta_{0}+9 \dot{\gamma} \hat{a l} n_{1}^{4} n_{2} \eta_{0}-6 \dot{\gamma} a l n_{1}^{4} n_{2}^{2} n_{2} \eta_{0}-$

$\left.3 \dot{\gamma}^{2} n_{1} n_{2} \eta_{0} \theta_{0}-6 \dot{\gamma}^{2} \hat{a} n_{1} n_{2} \eta_{0} \theta_{0}+2 \dot{\gamma}^{2} \hat{\beta} n_{1} n_{2} \eta_{0} \theta_{0}+9 \dot{\gamma}^{2} n_{1}^{3} n_{2} \eta_{0} \theta_{0}+12 \dot{\gamma}^{2} \hat{a} n_{1}^{3} n_{2} \eta_{0} \theta_{0}-6 \dot{\gamma}^{2} \hat{\beta} n_{1}^{3} n_{2} \eta_{0} \theta_{0}\right) /$ 
$\sigma_{22} \rightarrow$

$-\left(6 \dot{\gamma} \eta_{0}+6 \dot{\gamma} \hat{a} \eta_{0}-9 \dot{\gamma} n_{1}^{2} \eta_{0}-9 \dot{\gamma} \hat{l} n_{1}^{2} \eta_{0}+3 \dot{\gamma} n_{1}^{4} \eta_{0}+3 \dot{\gamma} \hat{a} n_{1}^{4} \eta_{0}-12 \dot{\gamma} n_{1}^{2} n_{2}^{2} \eta_{0}-12 \dot{\gamma}\right.$ al $n_{1}^{2} n_{2}^{2} \eta_{0}+$ $8 \dot{\gamma} \hat{\beta} n_{1}^{2} n_{2}^{2} \eta_{0}+6 \dot{\gamma} n_{1}^{4} n_{2}^{2} \eta_{0}+6 \dot{\gamma} \hat{a} l n_{1}^{4} n_{2}^{2} \eta_{0}-4 \dot{\gamma} \hat{\beta} n_{1}^{4} n_{2}^{2} \eta_{0}+12 \dot{\gamma} n_{1}^{2} r_{1} \eta_{0}+12 \dot{\gamma}$ al $n_{1}^{2} r_{1} \eta_{0}-12 \dot{\gamma} n_{1}^{4} r_{1} \eta_{0}-$ $12 \dot{\gamma} a \hat{l} n_{1}^{4} n_{1} \eta_{0}-6 \dot{\gamma} n_{1}^{2} n_{2}^{2} n_{1} \eta_{0}-12 \dot{\gamma}$ âl $n_{1}^{2} n_{2}^{2} n_{1} \eta_{0}+4 \dot{\gamma} \hat{\beta} n_{1}^{2} n_{2}^{2} n_{1} \eta_{0}-6 \dot{\gamma} n_{1}^{4} n_{2}^{2} n_{1} \eta_{0}+4 \dot{\gamma} \hat{\beta} n_{1}^{4} n_{2}^{2} n_{1} \eta_{0}-$ $3 \dot{\gamma} n_{1}^{2} r_{2} \eta_{0}-3 \dot{\gamma} \hat{a l} n_{1}^{2} r_{2} \eta_{0}+9 \dot{\gamma} n_{1}^{4} r_{2} \eta_{0}+9 \dot{\gamma} \hat{a l} n_{1}^{4} r_{2} \eta_{0}-6 \dot{\gamma} \hat{a} n_{1}^{4} n_{2}^{2} r_{2} \eta_{0}-3 \dot{\gamma}^{2} n_{1} n_{2} \eta_{0} \theta_{0}-$ $\left.6 \dot{\gamma}^{2} a \hat{l} n_{1} n_{2} \eta_{0} \theta_{0}+2 \dot{\gamma}^{2} \hat{\beta} n_{1} n_{2} \eta_{0} \theta_{0}+9 \dot{\gamma}^{2} n_{1}^{3} n_{2} \eta_{0} \theta_{0}+12 \dot{\gamma}^{2} a \hat{l} n_{1}^{3} n_{2} \eta_{0} \theta_{0}-6 \dot{\gamma}^{2} \hat{\beta} n_{1}^{3} n_{2} \eta_{0} \theta_{0}\right) /$ $\left(12 n_{1} n_{2}-18 n_{1}^{3} n_{2}+6 n_{1}^{5} n_{2}-12 n_{1} n_{2}^{3}+6 n_{1}^{3} n_{2}^{3}-12 n_{1} n_{2} r_{1}+42 n_{1}^{3} n_{2} r_{1}-30 n_{1}^{5} n_{2} r_{1}+30 n_{1} n_{2}^{3} r_{1}-\right.$ $30 n_{1}^{3} n_{2}^{3} r_{1}-24 n_{1}^{3} n_{2} r_{1}^{2}+24 n_{1}^{5} n_{2} r_{1}^{2}+24 n_{1}^{3} n_{2}^{3} r_{1}^{2}-6 n_{1}^{3} n_{2} r_{2}+18 n_{1}^{5} n_{2} r_{2}-12 n_{1} n_{2}^{3} r_{2}+18 n_{1}^{3} n_{2}^{3} r_{2}+$ $6 n_{1}^{3} n_{2} r_{1} r_{2}-18 n_{1}^{5} n_{2} n_{1} n_{2}-6 n_{1} n_{2}^{3} n_{1} r_{2}-18 n_{1}^{3} n_{2}^{3} n_{1} n_{2}+6 \dot{\gamma} \theta_{0}-9 \dot{\gamma} n_{1}^{2} \theta_{0}+3 \dot{\gamma} n_{1}^{4} \theta_{0}+3 \dot{\gamma} n_{2}^{2} \theta_{0}+$ $\left.3 \dot{\gamma} n_{1}^{2} n_{2}^{2} \theta_{0}+12 \dot{\gamma} n_{1}^{2} r_{1} \theta_{0}-12 \dot{\gamma} n_{1}^{4} r_{1} \theta_{0}-12 \dot{\gamma} n_{1}^{2} n_{2}^{2} r_{1} \theta_{0}-3 \dot{\gamma} n_{1}^{2} r_{2} \theta_{0}+9 \dot{\gamma} n_{1}^{4} r_{2} \theta_{0}-3 \dot{\gamma} n_{2}^{2} r_{2} \theta_{0}+9 \dot{\gamma} n_{1}^{2} n_{2}^{2} r_{2} \theta_{0}\right)+$ $\left(\left(-36 \dot{\gamma} \eta_{0}-36 \dot{\gamma}\right.\right.$ all $\eta_{0}+54 \dot{\gamma} n_{1}^{2} \eta_{0}+54 \dot{\gamma}$ all $n_{1}^{2} \eta_{0}-18 \dot{\gamma} n_{1}^{4} \eta_{0}-18 \dot{\gamma}$ âl $n_{1}^{4} \eta_{0}+54 \dot{\gamma} n_{2}^{2} \eta_{0}+54 \dot{\gamma}$ all $n_{2}^{2} \eta_{0}-$ $36 \dot{\gamma} n_{1}^{2} n_{2}^{2} \eta_{0}-72 \dot{\gamma} \hat{a l} n_{1}^{2} n_{2}^{2} \eta_{0}-24 \dot{\gamma} \hat{\beta} n_{1}^{2} n_{2}^{2} \eta_{0}+72 \dot{\gamma} n_{1}^{4} n_{2}^{2} \eta_{0}+144 \dot{\gamma} \hat{l} n_{1}^{4} n_{2}^{2} \eta_{0}-24 \dot{\gamma} \hat{\beta} n_{1}^{4} n_{2}^{2} \eta_{0}-$ $72 \dot{\gamma} n_{1}^{6} n_{2}^{2} \eta_{0}-108 \dot{\gamma} \hat{a l} n_{1}^{6} n_{2}^{2} \eta_{0}+48 \dot{\gamma} \hat{\beta} n_{1}^{6} n_{2}^{2} \eta_{0}-18 \dot{\gamma} n_{2}^{4} \eta_{0}-18 \dot{\gamma} \hat{a l} n_{2}^{4} \eta_{0}+72 \dot{\gamma} n_{1}^{2} n_{2}^{4} \eta_{0}+$ $144 \dot{\gamma} \hat{a} n_{1}^{2} n_{2}^{4} \eta_{0}-24 \dot{\gamma} \hat{\beta} n_{1}^{2} n_{2}^{4} \eta_{0}-144 \dot{\gamma} n_{1}^{4} n_{2}^{4} \eta_{0}-216 \dot{\gamma} \hat{a} 1 n_{1}^{4} n_{2}^{4} \eta_{0}+96 \dot{\gamma} \hat{\beta} n_{1}^{4} n_{2}^{4} \eta_{0}-72 \dot{\gamma} n_{1}^{2} n_{2}^{6} \eta_{0}-$ $108 \dot{\gamma} \hat{a} n_{1}^{2} n_{2}^{6} \eta_{0}+48 \dot{\gamma} \hat{\beta} n_{1}^{2} n_{2}^{6} \eta_{0}-72 \dot{\gamma} n_{1}^{2} r_{1} \eta_{0}-72 \dot{\gamma} \hat{a} l n_{1}^{2} r_{1} \eta_{0}+72 \dot{\gamma} n_{1}^{4} r_{1} \eta_{0}+72 \dot{\gamma}$ al $n_{1}^{4} r_{1} \eta_{0}-$ $72 \dot{\gamma} n_{2}^{2} r_{1} \eta_{0}-72 \dot{\gamma} a \hat{l} n_{2}^{2} r_{1} \eta_{0}+288 \dot{\gamma} n_{1}^{2} n_{2}^{2} r_{1} \eta_{0}+360 \dot{\gamma} a \hat{l} n_{1}^{2} n_{2}^{2} r_{1} n_{0}-48 \dot{\gamma} \hat{\beta} n_{1}^{2} n_{2}^{2} r_{1} \eta_{0}-$ $288 \dot{\gamma} n_{1}^{4} n_{2}^{2} r_{1} \eta_{0}-468 \dot{\gamma} \hat{l} n_{1}^{4} n_{2}^{2} n_{1} \eta_{0}+72 \dot{\gamma} \hat{\beta} n_{1}^{4} n_{2}^{2} r_{1} \eta_{0}+144 \dot{\gamma} n_{1}^{6} n_{2}^{2} n_{1} \eta_{0}+252 \dot{\gamma} \hat{l} n_{1}^{6} n_{2}^{2} n_{1} \eta_{0}-$ $96 \dot{\gamma} \hat{\beta} n_{1}^{6} n_{2}^{2} r_{1} \eta_{0}+72 \dot{\gamma} n_{2}^{4} r_{1} \eta_{0}+72 \dot{\gamma} \hat{a} n_{2}^{4} r_{1} \eta_{0}-288 \dot{\gamma} n_{1}^{2} n_{2}^{4} r_{1} \eta_{0}-468 \dot{\gamma} \hat{l} n_{1}^{2} n_{2}^{4} r_{1} \eta_{0}+$ $72 \dot{\gamma} \hat{\beta} n_{1}^{2} n_{2}^{4} r_{1} \eta_{0}+288 \dot{\gamma} n_{1}^{4} n_{2}^{4} n_{1} \eta_{0}+504 \dot{\gamma} a 1 n_{1}^{4} n_{2}^{4} r_{1} \eta_{0}-192 \dot{\gamma} \hat{\beta} n_{1}^{4} n_{2}^{4} n_{1} \eta_{0}+144 \dot{\gamma} n_{1}^{2} n_{2}^{6} r_{1} \eta_{0}+$ $252 \dot{\gamma} \hat{a} n_{1}^{2} n_{2}^{6} r_{1} \eta_{0}-96 \dot{\gamma} \hat{\beta} n_{1}^{2} n_{2}^{6} r_{1} \eta_{0}-144 \dot{\gamma} n_{1}^{2} n_{2}^{2} r_{1}^{2} \eta_{0}-144 \dot{\gamma}$ al $n_{1}^{2} n_{2}^{2} r_{1}^{2} \eta_{0}+216 \dot{\gamma} n_{1}^{4} n_{2}^{2} r_{1}^{2} \eta_{0}+$ $288 \dot{\gamma} \hat{a} l n_{1}^{4} n_{2}^{2} r_{1}^{2} \eta_{0}-48 \dot{\gamma} \hat{\beta} n_{1}^{4} n_{2}^{2} r_{1}^{2} \eta_{0}-72 \dot{\gamma} n_{1}^{6} n_{2}^{2} r_{1}^{2} \eta_{0}-144 \dot{\gamma} \hat{l} n_{1}^{6} n_{2}^{2} r_{1}^{2} \eta_{0}+48 \dot{\gamma} \hat{\beta} n_{1}^{6} n_{2}^{2} r_{1}^{2} \eta_{0}+$ $216 \dot{\gamma} n_{1}^{2} n_{2}^{4} r_{1}^{2} \eta_{0}+288 \dot{\gamma} \hat{l} n_{1}^{2} n_{2}^{4} r_{1}^{2} \eta_{0}-48 \dot{\gamma} \hat{\beta} n_{1}^{2} n_{2}^{4} r_{1}^{2} \eta_{0}-144 \dot{\gamma} n_{1}^{4} n_{2}^{4} r_{1}^{2} \eta_{0}-288 \dot{\gamma}$ al $n_{1}^{4} n_{2}^{4} r_{1}^{2} \eta_{0}+$ $96 \dot{\gamma} \hat{\beta} n_{1}^{4} n_{2}^{4} r_{1}^{2} \eta_{0}-72 \dot{\gamma} n_{1}^{2} n_{2}^{6} r_{1}^{2} \eta_{0}-144 \dot{\gamma} \hat{a} n_{1}^{2} n_{2}^{6} r_{1}^{2} \eta_{0}+48 \dot{\gamma} \hat{\beta} n_{1}^{2} n_{2}^{6} r_{1}^{2} \eta_{0}+18 \dot{\gamma} n_{1}^{2} r_{2} \eta_{0}+$ $18 \dot{\gamma} \hat{a} n_{1}^{2} r_{2} \eta_{0}-54 \dot{\gamma} n_{1}^{4} r_{2} \eta_{0}-54 \dot{\gamma}$ all $n_{1}^{4} r_{2} n_{0}+18 \dot{\gamma} n_{2}^{2} r_{2} \eta_{0}+18 \dot{\gamma}$ al $n_{2}^{2} r_{2} n_{0}-72 \dot{\gamma} n_{1}^{2} n_{2}^{2} r_{2} n_{0}-$ $72 \dot{\gamma} a \hat{l} n_{1}^{2} n_{2}^{2} r_{2} \eta_{0}+108 \dot{\gamma} n_{1}^{4} n_{2}^{2} r_{2} \eta_{0}+144 \dot{\gamma}$ al $n_{1}^{4} n_{2}^{2} r_{2} \eta_{0}-36 \dot{\gamma}$ âl $n_{1}^{6} n_{2}^{2} r_{2} \eta_{0}-54 \dot{\gamma} n_{2}^{4} r_{2} \eta_{0}-$ $54 \dot{\gamma} \hat{a} 1 n_{2}^{4} r_{2} \eta_{0}+108 \dot{\gamma} n_{1}^{2} n_{2}^{4} r_{2} \eta_{0}+144 \dot{\gamma}$ al $n_{1}^{2} n_{2}^{4} r_{2} \eta_{0}-72 \dot{\gamma} \hat{a l} n_{1}^{4} n_{2}^{4} r_{2} \eta_{0}-36 \dot{\gamma}$ al $n_{1}^{2} n_{2}^{6} r_{2} \eta_{0}+$ $72 \dot{\gamma} n_{1}^{2} n_{2}^{2} r_{1} r_{2} \eta_{0}+72 \dot{\gamma} \hat{a l} n_{1}^{2} n_{2}^{2} r_{1} r_{2} \eta_{0}-108 \dot{\gamma} n_{1}^{4} n_{2}^{2} r_{1} r_{2} \eta_{0}-108 \dot{\gamma} \hat{a} n_{1}^{4} n_{2}^{2} r_{1} r_{2} \eta_{0}+$ $36 \dot{\gamma} \hat{a l} n_{1}^{6} n_{2}^{2} r_{1} r_{2} \eta_{0}-108 \dot{\gamma} n_{1}^{2} n_{2}^{4} r_{1} r_{2} \eta_{0}-108 \dot{\gamma} \hat{a} n_{1}^{2} n_{2}^{4} r_{1} r_{2} \eta_{0}+72 \dot{\gamma} \hat{l} n_{1}^{4} n_{2}^{4} r_{1} r_{2} \eta_{0}+$ $36 \dot{\gamma} \hat{a} n_{1}^{2} n_{2}^{6} r_{1} r_{2} \eta_{0}-18 \dot{\gamma}^{2} n_{1}^{3} n_{2} \eta_{0} \theta_{0}+12 \dot{\gamma}^{2} \hat{\beta} n_{1}^{3} n_{2} \eta_{0} \theta_{0}-36 \dot{\gamma}^{2} n_{1}^{5} n_{2} \eta_{0} \theta_{0}-54 \dot{\gamma}^{2} \hat{l} n_{1}^{5} n_{2} \eta_{0} \theta_{0}+$ $24 \dot{\gamma}^{2} \hat{\beta} n_{1}^{5} n_{2} \eta_{0} \theta_{0}+18 \dot{\gamma}^{2} n_{1} n_{2}^{3} \eta_{0} \theta_{0}-12 \dot{\gamma}^{2} \hat{\beta} n_{1} n_{2}^{3} \eta_{0} \theta_{0}+36 \dot{\gamma}^{2} n_{1} n_{2}^{5} \eta_{0} \theta_{0}+54 \dot{\gamma}^{2}$ al $n_{1} n_{2}^{5} \eta_{0} \theta_{0}-$ $24 \dot{\gamma}^{2} \hat{\beta} n_{1} n_{2}^{5} \eta_{0} \theta_{0}-36 \dot{\gamma}^{2} n_{1}^{3} n_{2} r_{1} \eta_{0} \theta_{0}-72 \dot{\gamma}^{2} a 1 n_{1}^{3} n_{2} r_{1} \eta_{0} \theta_{0}+24 \dot{\gamma}^{2} \hat{\beta} n_{1}^{3} n_{2} r_{1} \eta_{0} \theta_{0}+36 \dot{\gamma}^{2} n_{1}^{5} n_{2} r_{1} \eta_{0} \theta_{0}+$ $72 \dot{\gamma}^{2} \hat{a} n_{1}^{5} n_{2} r_{1} \eta_{0} \theta_{0}-24 \dot{\gamma}^{2} \hat{\beta} n_{1}^{5} n_{2} r_{1} \eta_{0} \theta_{0}+36 \dot{\gamma}^{2} n_{1} n_{2}^{3} r_{1} \eta_{0} \theta_{0}+72 \dot{\gamma}^{2}$ al $n_{1} n_{2}^{3} r_{1} \eta_{0} \theta_{0}-$ $24 \dot{\gamma}^{2} \hat{\beta} n_{1} n_{2}^{3} n_{1} \eta_{0} \theta_{0}-36 \dot{\gamma}^{2} n_{1} n_{2}^{5} n_{1} \eta_{0} \theta_{0}-72 \dot{\gamma}^{2} a \hat{l} n_{1} n_{2}^{5} n_{1} \eta_{0} \theta_{0}+24 \dot{\gamma}^{2} \hat{\beta} n_{1} n_{2}^{5} n_{1} \eta_{0} \theta_{0}-$ $18 \dot{\gamma}^{2}$ âl $n_{1}^{5} n_{2} r_{2} \eta_{0} \theta_{0}+18 \dot{\gamma}^{2}$ all $\left.n_{1} n_{2}^{5} r_{2} \eta_{0} \theta_{0}\right)$

$\left(-12 r_{1}+18 n_{1}^{2} r_{1}-6 n_{1}^{4} r_{1}+12 n_{1}^{2} n_{2}^{2} r_{1}-24 n_{1}^{2} r_{1}^{2}+24 n_{1}^{4} r_{1}^{2}+24 n_{1}^{2} n_{2}^{2} r_{1}^{2}-24 n_{1}^{2} n_{2}^{2} r_{2}+6 n_{1}^{2} r_{1} r_{2}-\right.$ $\left.\left.18 n_{1}^{4} r_{1} r_{2}-12 n_{1}^{2} n_{2}^{2} n_{1} r_{2}+6 \dot{\gamma} n_{1} n_{2} \theta_{0}-6 \dot{\gamma} n_{1} n_{2} r_{2} \theta_{0}-6 \dot{\gamma}^{2} \theta_{0}^{2}\right)\right) /$

$\left(\left(12 n_{1} n_{2}-18 n_{1}^{3} n_{2}+6 n_{1}^{5} n_{2}-12 n_{1} n_{2}^{3}+6 n_{1}^{3} n_{2}^{3}-12 n_{1} n_{2} n_{1}+42 n_{1}^{3} n_{2} n_{1}-30 n_{1}^{5} n_{2} n_{1}+30 n_{1} n_{2}^{3} n_{1}-\right.\right.$ $30 n_{1}^{3} n_{2}^{3} r_{1}-24 n_{1}^{3} n_{2} r_{1}^{2}+24 n_{1}^{5} n_{2} r_{1}^{2}+24 n_{1}^{3} n_{2}^{3} r_{1}^{2}-6 n_{1}^{3} n_{2} r_{2}+18 n_{1}^{5} n_{2} r_{2}-12 n_{1} n_{2}^{3} r_{2}+18 n_{1}^{3} n_{2}^{3} r_{2}+$ $6 n_{1}^{3} n_{2} r_{1} r_{2}-18 n_{1}^{5} n_{2} r_{1} r_{2}-6 n_{1} n_{2}^{3} r_{1} r_{2}-18 n_{1}^{3} n_{2}^{3} r_{1} r_{2}+6 \dot{\gamma} \theta_{0}-9 \dot{\gamma} n_{1}^{2} \theta_{0}+3 \dot{\gamma} n_{1}^{4} \theta_{0}+3 \dot{\gamma} n_{2}^{2} \theta_{0}+$ $\left.3 \dot{\gamma} n_{1}^{2} n_{2}^{2} \theta_{0}+12 \dot{\gamma} n_{1}^{2} r_{1} \theta_{0}-12 \dot{\gamma} n_{1}^{4} r_{1} \theta_{0}-12 \dot{\gamma} n_{1}^{2} n_{2}^{2} r_{1} \theta_{0}-3 \dot{\gamma} n_{1}^{2} r_{2} \theta_{0}+9 \dot{\gamma} n_{1}^{4} r_{2} \theta_{0}-3 \dot{\gamma} n_{2}^{2} r_{2} \theta_{0}+9 \dot{\gamma} n_{1}^{2} n_{2}^{2} r_{2} \theta_{0}\right)$ $\left(-72 n_{1}^{2} n_{2}^{2}+144 n_{1}^{4} n_{2}^{2}-72 n_{1}^{6} n_{2}^{2}+144 n_{1}^{2} n_{2}^{4}-144 n_{1}^{4} n_{2}^{4}-72 n_{1}^{2} n_{2}^{6}+72 r_{1}-108 n_{1}^{2} r_{1}+36 n_{1}^{4} r_{1}-108 n_{2}^{2} r_{1}+\right.$ $288 n_{1}^{2} n_{2}^{2} r_{1}-648 n_{1}^{4} n_{2}^{2} r_{1}+432 n_{1}^{6} n_{2}^{2} r_{1}+36 n_{2}^{4} r_{1}-648 n_{1}^{2} n_{2}^{4} r_{1}+864 n_{1}^{4} n_{2}^{4} r_{1}+432 n_{1}^{2} n_{2}^{6} r_{1}+144 n_{1}^{2} r_{1}^{2}-$ $144 n_{1}^{4} r_{1}^{2}+144 n_{2}^{2} r_{1}^{2}-720 n_{1}^{2} n_{2}^{2} r_{1}^{2}+1080 n_{1}^{4} n_{2}^{2} r_{1}^{2}-648 n_{1}^{6} n_{2}^{2} r_{1}^{2}-144 n_{2}^{4} r_{1}^{2}+1080 n_{1}^{2} n_{2}^{4} r_{1}^{2}-1296 n_{1}^{4} n_{2}^{4} r_{1}^{2}-$ $648 n_{1}^{2} n_{2}^{6} r_{1}^{2}+288 n_{1}^{2} n_{2}^{2} r_{1}^{3}-576 n_{1}^{4} n_{2}^{2} r_{1}^{3}+288 n_{1}^{6} n_{2}^{2} r_{1}^{3}-576 n_{1}^{2} n_{2}^{4} r_{1}^{3}+576 n_{1}^{4} n_{2}^{4} r_{1}^{3}+288 n_{1}^{2} n_{2}^{6} r_{1}^{3}+$ $72 n_{1}^{2} n_{2}^{2} r_{2}+144 n_{1}^{4} n_{2}^{2} r_{2}-216 n_{1}^{6} n_{2}^{2} r_{2}+144 n_{1}^{2} n_{2}^{4} r_{2}-432 n_{1}^{4} n_{2}^{4} r_{2}-$ 
$216 n_{1}^{2} n_{2}^{6} r_{2}-36 n_{1}^{2} r_{1} r_{2}+108 n_{1}^{4} r_{1} r_{2}-36 n_{2}^{2} r_{1} r_{2}+288 n_{1}^{2} n_{2}^{2} r_{1} r_{2}-504 n_{1}^{4} n_{2}^{2} r_{1} r_{2}+432 n_{1}^{6} n_{2}^{2} r_{1} r_{2}+$ $108 n_{2}^{4} r_{1} r_{2}-504 n_{1}^{2} n_{2}^{4} r_{1} r_{2}+864 n_{1}^{4} n_{2}^{4} r_{1} r_{2}+432 n_{1}^{2} n_{2}^{6} r_{1} r_{2}-144 n_{1}^{2} n_{2}^{2} r_{1}^{2} r_{2}+360 n_{1}^{4} n_{2}^{2} r_{1}^{2} r_{2}-$ $216 n_{1}^{6} n_{2}^{2} r_{1}^{2} r_{2}+360 n_{1}^{2} n_{2}^{4} r_{1}^{2} r_{2}-432 n_{1}^{4} n_{2}^{4} r_{1}^{2} r_{2}-216 n_{1}^{2} n_{2}^{6} r_{1}^{2} r_{2}+72 \dot{\gamma}^{2} \theta_{0}^{2}-36 \dot{\gamma}^{2} n_{1}^{2} \theta_{0}^{2}+18 \dot{\gamma}^{2} n_{1}^{4} \theta_{0}^{2}-$

$36 \dot{\gamma}^{2} n_{2}^{2} \theta_{0}^{2}+36 \dot{\gamma}^{2} n_{1}^{2} n_{2}^{2} \theta_{0}^{2}+18 \dot{\gamma}^{2} n_{2}^{4} \theta_{0}^{2}+72 \dot{\gamma}^{2} n_{1}^{2} r_{1} \theta_{0}^{2}-72 \dot{\gamma}^{2} n_{1}^{4} r_{1} \theta_{0}^{2}+72 \dot{\gamma}^{2} n_{2}^{2} r_{1} \theta_{0}^{2}-144 \dot{\gamma}^{2} n_{1}^{2} n_{2}^{2} r_{1} \theta_{0}^{2}-$

$\left.\left.72 \dot{\gamma}^{2} n_{2}^{4} r_{1} \theta_{0}^{2}-36 \dot{\gamma}^{2} n_{1}^{2} r_{2} \theta_{0}^{2}+54 \dot{\gamma}^{2} n_{1}^{4} r_{2} \theta_{0}^{2}-36 \dot{\gamma}^{2} n_{2}^{2} r_{2} \theta_{0}^{2}+108 \dot{\gamma}^{2} n_{1}^{2} n_{2}^{2} r_{2} \theta_{0}^{2}+54 \dot{\gamma}^{2} n_{2}^{4} r_{2} \theta_{0}^{2}\right)\right)$ ， $\sigma_{12} \rightarrow$

$-\left(-36 \dot{\gamma} \eta_{0}-36 \dot{\gamma} \hat{a} \eta_{0}+54 \dot{\gamma} n_{1}^{2} \eta_{0}+54 \dot{\gamma} \hat{a} n_{1}^{2} \eta_{0}-18 \dot{\gamma} n_{1}^{4} \eta_{0}-18 \dot{\gamma}\right.$ al $n_{1}^{4} \eta_{0}+54 \dot{\gamma} n_{2}^{2} \eta_{0}+54 \dot{\gamma}$ âl $n_{2}^{2} \eta_{0}-$ $36 \dot{\gamma} n_{1}^{2} n_{2}^{2} \eta_{0}-72 \dot{\gamma} \hat{a} n_{1}^{2} n_{2}^{2} \eta_{0}-24 \dot{\gamma} \hat{\beta} n_{1}^{2} n_{2}^{2} \eta_{0}+72 \dot{\gamma} n_{1}^{4} n_{2}^{2} \eta_{0}+144 \dot{\gamma} \hat{a l} n_{1}^{4} n_{2}^{2} \eta_{0}-24 \dot{\gamma} \hat{\beta} n_{1}^{4} n_{2}^{2} \eta_{0}-$ $72 \dot{\gamma} n_{1}^{6} n_{2}^{2} \eta_{0}-108 \dot{\gamma} \hat{a} n_{1}^{6} n_{2}^{2} \eta_{0}+48 \dot{\gamma} \hat{\beta} n_{1}^{6} n_{2}^{2} \eta_{0}-18 \dot{\gamma} n_{2}^{4} \eta_{0}-18 \dot{\gamma} \hat{a} 1 n_{2}^{4} \eta_{0}+72 \dot{\gamma} n_{1}^{2} n_{2}^{4} \eta_{0}+$ $144 \dot{\gamma} \hat{a} n_{1}^{2} n_{2}^{4} \eta_{0}-24 \dot{\gamma} \hat{\beta} n_{1}^{2} n_{2}^{4} \eta_{0}-144 \dot{\gamma} n_{1}^{4} n_{2}^{4} \eta_{0}-216 \dot{\gamma} \hat{a} n_{1}^{4} n_{2}^{4} \eta_{0}+96 \dot{\gamma} \hat{\beta} n_{1}^{4} n_{2}^{4} \eta_{0}-72 \dot{\gamma} n_{1}^{2} n_{2}^{6} \eta_{0}-$ $108 \dot{\gamma} \hat{a} 1 n_{1}^{2} n_{2}^{6} \eta_{0}+48 \dot{\gamma} \hat{\beta} n_{1}^{2} n_{2}^{6} \eta_{0}-72 \dot{\gamma} n_{1}^{2} n_{1} \eta_{0}-72 \dot{\gamma} a \hat{l} n_{1}^{2} n_{1} \eta_{0}+72 \dot{\gamma} n_{1}^{4} n_{1} \eta_{0}+72 \dot{\gamma}$ âl $n_{1}^{4} n_{1} \eta_{0}-$ $72 \dot{\gamma} n_{2}^{2} r_{1} \eta_{0}-72 \dot{\gamma} \hat{a} 1 n_{2}^{2} r_{1} \eta_{0}+288 \dot{\gamma} n_{1}^{2} n_{2}^{2} r_{1} \eta_{0}+360 \dot{\gamma}$ al $n_{1}^{2} n_{2}^{2} r_{1} \eta_{0}-48 \dot{\gamma} \hat{\beta} n_{1}^{2} n_{2}^{2} r_{1} \eta_{0}-$ $288 \dot{\gamma} n_{1}^{4} n_{2}^{2} r_{1} \eta_{0}-468 \dot{\gamma}$ all $n_{1}^{4} n_{2}^{2} r_{1} \eta_{0}+72 \dot{\gamma} \hat{\beta} n_{1}^{4} n_{2}^{2} r_{1} \eta_{0}+144 \dot{\gamma} n_{1}^{6} n_{2}^{2} r_{1} \eta_{0}+252 \dot{\gamma}$ al $n_{1}^{6} n_{2}^{2} r_{1} \eta_{0}-$ $96 \dot{\gamma} \hat{\beta} n_{1}^{6} n_{2}^{2} n_{1} \eta_{0}+72 \dot{\gamma} n_{2}^{4} n_{1} \eta_{0}+72 \dot{\gamma} a \hat{l} n_{2}^{4} n_{1} \eta_{0}-288 \dot{\gamma} n_{1}^{2} n_{2}^{4} r_{1} \eta_{0}-468 \dot{\gamma}$ al $n_{1}^{2} n_{2}^{4} n_{1} \eta_{0}+$ $72 \dot{\gamma} \hat{\beta} n_{1}^{2} n_{2}^{4} n_{1} \eta_{0}+288 \dot{\gamma} n_{1}^{4} n_{2}^{4} n_{1} \eta_{0}+504 \dot{\gamma} \hat{a} n_{1}^{4} n_{2}^{4} n_{1} \eta_{0}-192 \dot{\gamma} \hat{\beta} n_{1}^{4} n_{2}^{4} n_{1} \eta_{0}+144 \dot{\gamma} n_{1}^{2} n_{2}^{6} n_{1} \eta_{0}+$ $252 \dot{\gamma} \hat{a} 1 n_{1}^{2} n_{2}^{6} r_{1} \eta_{0}-96 \dot{\gamma} \hat{\beta} n_{1}^{2} n_{2}^{6} r_{1} \eta_{0}-144 \dot{\gamma} n_{1}^{2} n_{2}^{2} r_{1}^{2} \eta_{0}-144 \dot{\gamma}$ al $n_{1}^{2} n_{2}^{2} r_{1}^{2} \eta_{0}+216 \dot{\gamma} n_{1}^{4} n_{2}^{2} r_{1}^{2} \eta_{0}+$ $288 \dot{\gamma} \hat{a} n_{1}^{4} n_{2}^{2} r_{1}^{2} \eta_{0}-48 \dot{\gamma} \hat{\beta} n_{1}^{4} n_{2}^{2} r_{1}^{2} \eta_{0}-72 \dot{\gamma} n_{1}^{6} n_{2}^{2} r_{1}^{2} \eta_{0}-144 \dot{\gamma}$ al $n_{1}^{6} n_{2}^{2} r_{1}^{2} \eta_{0}+48 \dot{\gamma} \hat{\beta} n_{1}^{6} n_{2}^{2} r_{1}^{2} \eta_{0}+$ $216 \dot{\gamma} n_{1}^{2} n_{2}^{4} r_{1}^{2} \eta_{0}+288 \dot{\gamma} \hat{a} n_{1}^{2} n_{2}^{4} r_{1}^{2} \eta_{0}-48 \dot{\gamma} \hat{\beta} n_{1}^{2} n_{2}^{4} r_{1}^{2} \eta_{0}-144 \dot{\gamma} n_{1}^{4} n_{2}^{4} r_{1}^{2} \eta_{0}-288 \dot{\gamma}$ al $n_{1}^{4} n_{2}^{4} r_{1}^{2} \eta_{0}+$ $96 \dot{\gamma} \hat{\beta} n_{1}^{4} n_{2}^{4} r_{1}^{2} \eta_{0}-72 \dot{\gamma} n_{1}^{2} n_{2}^{6} r_{1}^{2} \eta_{0}-144 \dot{\gamma} \hat{a} 1 n_{1}^{2} n_{2}^{6} r_{1}^{2} \eta_{0}+48 \dot{\gamma} \hat{\beta} n_{1}^{2} n_{2}^{6} r_{1}^{2} \eta_{0}+18 \dot{\gamma} n_{1}^{2} r_{2} \eta_{0}+$ $18 \dot{\gamma}$ al $n_{1}^{2} r_{2} \eta_{0}-54 \dot{\gamma} n_{1}^{4} r_{2} \eta_{0}-54 \dot{\gamma}$ all $n_{1}^{4} r_{2} \eta_{0}+18 \dot{\gamma} n_{2}^{2} r_{2} \eta_{0}+18 \dot{\gamma}$ âl $n_{2}^{2} r_{2} \eta_{0}-72 \dot{\gamma} n_{1}^{2} n_{2}^{2} r_{2} \eta_{0}-$ $72 \dot{\gamma} a \hat{l} n_{1}^{2} n_{2}^{2} r_{2} \eta_{0}+108 \dot{\gamma} n_{1}^{4} n_{2}^{2} r_{2} \eta_{0}+144 \dot{\gamma} a 1 n_{1}^{4} n_{2}^{2} r_{2} \eta_{0}-36 \dot{\gamma}$ all $n_{1}^{6} n_{2}^{2} r_{2} \eta_{0}-54 \dot{\gamma} n_{2}^{4} r_{2} \eta_{0}-$ $54 \dot{\gamma} a \hat{l} n_{2}^{4} r_{2} \eta_{0}+108 \dot{\gamma} n_{1}^{2} n_{2}^{4} r_{2} \eta_{0}+144 \dot{\gamma}$ all $n_{1}^{2} n_{2}^{4} r_{2} \eta_{0}-72 \dot{\gamma} \hat{a} n_{1}^{4} n_{2}^{4} r_{2} \eta_{0}-36 \dot{\gamma}$ all $n_{1}^{2} n_{2}^{6} r_{2} \eta_{0}+$ $72 \dot{\gamma} n_{1}^{2} n_{2}^{2} r_{1} r_{2} \eta_{0}+72 \dot{\gamma}$ all $n_{1}^{2} n_{2}^{2} r_{1} r_{2} \eta_{0}-108 \dot{\gamma} n_{1}^{4} n_{2}^{2} r_{1} r_{2} \eta_{0}-108 \dot{\gamma} a \hat{l} n_{1}^{4} n_{2}^{2} r_{1} r_{2} \eta_{0}+$ $36 \dot{\gamma} \hat{a} 1 n_{1}^{6} n_{2}^{2} r_{1} r_{2} \eta_{0}-108 \dot{\gamma} n_{1}^{2} n_{2}^{4} r_{1} r_{2} \eta_{0}-108 \dot{\gamma}$ al $n_{1}^{2} n_{2}^{4} r_{1} r_{2} \eta_{0}+72 \dot{\gamma} \hat{a l} n_{1}^{4} n_{2}^{4} r_{1} r_{2} \eta_{0}+$ $36 \dot{\gamma} \hat{a} n_{1}^{2} n_{2}^{6} r_{1} r_{2} \eta_{0}-18 \dot{\gamma}^{2} n_{1}^{3} n_{2} \eta_{0} \theta_{0}+12 \dot{\gamma}^{2} \hat{\beta} n_{1}^{3} n_{2} \eta_{0} \theta_{0}-36 \dot{\gamma}^{2} n_{1}^{5} n_{2} \eta_{0} \theta_{0}-54 \dot{\gamma}^{2} \hat{a} 1 n_{1}^{5} n_{2} \eta_{0} \theta_{0}+$ $24 \dot{\gamma}^{2} \hat{\beta} n_{1}^{5} n_{2} \eta_{0} \theta_{0}+18 \dot{\gamma}^{2} n_{1} n_{2}^{3} \eta_{0} \theta_{0}-12 \dot{\gamma}^{2} \hat{\beta} n_{1} n_{2}^{3} \eta_{0} \theta_{0}+36 \dot{\gamma}^{2} n_{1} n_{2}^{5} \eta_{0} \theta_{0}+54 \dot{\gamma}^{2} \hat{a} 1 n_{1} n_{2}^{5} \eta_{0} \theta_{0}-$ $24 \dot{\gamma}^{2} \hat{\beta} n_{1} n_{2}^{5} \eta_{0} \theta_{0}-36 \dot{\gamma}^{2} n_{1}^{3} n_{2} r_{1} \eta_{0} \theta_{0}-72 \dot{\gamma}^{2} \hat{a} 1 n_{1}^{3} n_{2} r_{1} \eta_{0} \theta_{0}+24 \dot{\gamma}^{2} \hat{\beta} n_{1}^{3} n_{2} r_{1} \eta_{0} \theta_{0}+36 \dot{\gamma}^{2} n_{1}^{5} n_{2} r_{1} \eta_{0} \theta_{0}+$ $72 \dot{\gamma}^{2} \hat{a} 1 n_{1}^{5} n_{2} r_{1} \eta_{0} \theta_{0}-24 \dot{\gamma}^{2} \hat{\beta} n_{1}^{5} n_{2} r_{1} \eta_{0} \theta_{0}+36 \dot{\gamma}^{2} n_{1} n_{2}^{3} r_{1} \eta_{0} \theta_{0}+72 \dot{\gamma}^{2} \hat{a} n_{1} n_{2}^{3} r_{1} \eta_{0} \theta_{0}-$ $24 \dot{\gamma}^{2} \hat{\beta} n_{1} n_{2}^{3} r_{1} \eta_{0} \theta_{0}-36 \dot{\gamma}^{2} n_{1} n_{2}^{5} r_{1} \eta_{0} \theta_{0}-72 \dot{\gamma}^{2} \hat{a} n_{1} n_{2}^{5} r_{1} \eta_{0} \theta_{0}+24 \dot{\gamma}^{2} \hat{\beta} n_{1} n_{2}^{5} n_{1} \eta_{0} \theta_{0}-$ $18 \dot{\gamma}^{2} \hat{a} \mathbf{l} n_{1}^{5} n_{2} r_{2} \eta_{0} \theta_{0}+18 \dot{\gamma}^{2}$ âl $\left.n_{1} n_{2}^{5} r_{2} \eta_{0} \theta_{0}\right) /$ $\left(-72 n_{1}^{2} n_{2}^{2}+144 n_{1}^{4} n_{2}^{2}-72 n_{1}^{6} n_{2}^{2}+144 n_{1}^{2} n_{2}^{4}-144 n_{1}^{4} n_{2}^{4}-72 n_{1}^{2} n_{2}^{6}+72 n_{1}-108 n_{1}^{2} r_{1}+36 n_{1}^{4} r_{1}-108 n_{2}^{2} r_{1}+\right.$ $288 n_{1}^{2} n_{2}^{2} r_{1}-648 n_{1}^{4} n_{2}^{2} r_{1}+432 n_{1}^{6} n_{2}^{2} r_{1}+36 n_{2}^{4} r_{1}-648 n_{1}^{2} n_{2}^{4} r_{1}+864 n_{1}^{4} n_{2}^{4} r_{1}+432 n_{1}^{2} n_{2}^{6} r_{1}+144 n_{1}^{2} r_{1}^{2}-$ $144 n_{1}^{4} r_{1}^{2}+144 n_{2}^{2} r_{1}^{2}-720 n_{1}^{2} n_{2}^{2} r_{1}^{2}+1080 n_{1}^{4} n_{2}^{2} r_{1}^{2}-648 n_{1}^{6} n_{2}^{2} r_{1}^{2}-144 n_{2}^{4} r_{1}^{2}+1080 n_{1}^{2} n_{2}^{4} r_{1}^{2}-1296 n_{1}^{4} n_{2}^{4} r_{1}^{2}-$ $648 n_{1}^{2} n_{2}^{6} r_{1}^{2}+288 n_{1}^{2} n_{2}^{2} r_{1}^{3}-576 n_{1}^{4} n_{2}^{2} r_{1}^{3}+288 n_{1}^{6} n_{2}^{2} r_{1}^{3}-576 n_{1}^{2} n_{2}^{4} r_{1}^{3}+576 n_{1}^{4} n_{2}^{4} r_{1}^{3}+288 n_{1}^{2} n_{2}^{6} r_{1}^{3}+$ $72 n_{1}^{2} n_{2}^{2} r_{2}+144 n_{1}^{4} n_{2}^{2} r_{2}-216 n_{1}^{6} n_{2}^{2} r_{2}+144 n_{1}^{2} n_{2}^{4} r_{2}-432 n_{1}^{4} n_{2}^{4} r_{2}-216 n_{1}^{2} n_{2}^{6} r_{2}-36 n_{1}^{2} r_{1} r_{2}+$ $108 n_{1}^{4} r_{1} r_{2}-36 n_{2}^{2} r_{1} r_{2}+288 n_{1}^{2} n_{2}^{2} r_{1} r_{2}-504 n_{1}^{4} n_{2}^{2} r_{1} r_{2}+432 n_{1}^{6} n_{2}^{2} r_{1} r_{2}+108 n_{2}^{4} r_{1} r_{2}-504 n_{1}^{2} n_{2}^{4} r_{1} r_{2}+$ $864 n_{1}^{4} n_{2}^{4} r_{1} r_{2}+432 n_{1}^{2} n_{2}^{6} r_{1} r_{2}-144 n_{1}^{2} n_{2}^{2} r_{1}^{2} r_{2}+360 n_{1}^{4} n_{2}^{2} r_{1}^{2} r_{2}-216 n_{1}^{6} n_{2}^{2} r_{1}^{2} r_{2}+360 n_{1}^{2} n_{2}^{4} r_{1}^{2} r_{2}-$ $432 n_{1}^{4} n_{2}^{4} r_{1}^{2} r_{2}-216 n_{1}^{2} n_{2}^{6} r_{1}^{2} r_{2}+72 \dot{\gamma}^{2} \theta_{0}^{2}-36 \dot{\gamma}^{2} n_{1}^{2} \theta_{0}^{2}+18 \dot{\gamma}^{2} n_{1}^{4} \theta_{0}^{2}-36 \dot{\gamma}^{2} n_{2}^{2} \theta_{0}^{2}+36 \dot{\gamma}^{2} n_{1}^{2} n_{2}^{2} \theta_{0}^{2}+$ $18 \dot{\gamma}^{2} n_{2}^{4} \theta_{0}^{2}+72 \dot{\gamma}^{2} n_{1}^{2} r_{1} \theta_{0}^{2}-72 \dot{\gamma}^{2} n_{1}^{4} r_{1} \theta_{0}^{2}+72 \dot{\gamma}^{2} n_{2}^{2} r_{1} \theta_{0}^{2}-144 \dot{\gamma}^{2} n_{1}^{2} n_{2}^{2} r_{1} \theta_{0}^{2}-72 \dot{\gamma}^{2} n_{2}^{4} r_{1} \theta_{0}^{2}-$ $\left.\left.\left.36 \dot{\gamma}^{2} n_{1}^{2} r_{2} \theta_{0}^{2}+54 \dot{\gamma}^{2} n_{1}^{4} r_{2} \theta_{0}^{2}-36 \dot{\gamma}^{2} n_{2}^{2} r_{2} \theta_{0}^{2}+108 \dot{\gamma}^{2} n_{1}^{2} n_{2}^{2} r_{2} \theta_{0}^{2}+54 \dot{\gamma}^{2} n_{2}^{4} r_{2} \theta_{0}^{2}\right)\right\}\right\}$ 